Louisiana State University

LSU Digital Commons

2009

\title{
Meso-scale heating predictions for weak impact of granular energetic solids
}

\author{
Rohan Panchadhara \\ Louisiana State University and Agricultural and Mechanical College
}

Follow this and additional works at: https://digitalcommons.Isu.edu/gradschool_dissertations

Part of the Mechanical Engineering Commons

\section{Recommended Citation}

Panchadhara, Rohan, "Meso-scale heating predictions for weak impact of granular energetic solids" (2009). LSU Doctoral Dissertations. 3467.

https://digitalcommons.Isu.edu/gradschool_dissertations/3467

This Dissertation is brought to you for free and open access by the Graduate School at LSU Digital Commons. It has been accepted for inclusion in LSU Doctoral Dissertations by an authorized graduate school editor of LSU Digital Commons. For more information, please contactgradetd@lsu.edu. 


\author{
A Dissertation \\ Submitted to the Graduate Faculty of the \\ Louisiana State University and \\ Agricultural and Mechanical College \\ in partial fulfillment of the \\ requirements for the degree of \\ Doctor of Philosophy \\ in
} The Department of Mechanical Engineering

by Rohan Panchadhara

B.Tech., Jawaharlal Nehru Technological University, 2003

August, 2009 


\section{Dedication}

This dissertation is dedicated to my parents Dileep and Anitha Panchadhara, to my sister Sneha Panchadhara and to my good friend Rajuli Lall, whose love and support made this graduate experience both enjoyable and rewarding. 


\section{Acknowledgments}

I would like to acknowledge several people who have contributed in several ways to make my graduate experience enjoyable and rewarding. First and foremost, I would like to acknowledge my advisor, Associate Prof. Keith Gonthier, for his technical expertise and guidance. Our countless discussions on technical as well as everyday issues have made me come to think of him as a colleague and a friend rather than a mentor. For this I would like to sincerely thank him. I would like to acknowledge Dr. Glenn Sinclair, Dr. Dimitris E. Nikitopoulos, Dr. Guoqiang Li and Dr. Michael M. Tom, Louisiana State University, for agreeing to be on my committee and taking the time to review this dissertation. I am also pleased to thank the faculty and staff of the mechanical engineering department, Louisiana State University, for their instruction, and their willingness to help solve various problems as they arose.

This is a great opportunity for me to express my gratitude to my colleagues and friends. I would like to acknowledge colleague Michael Crochet for his continuous support and encouragement, and for insights gained through many informal discussions of our research. I would also like to thank my colleagues Hemant Khatod, Blaise Paul, Sunada Chakravarthy and Anirban Mandal for making the workplace pleasant and productive. I am pleased to thank my good friends Vishnu Dutta, Sachin Singh and Anirban Mukherjee for their continuous support and inspiration. This dissertation would not have been possible without their encouragement.

I gratefully acknowledge funding for this work provided by the Mechanical Engineering Department, Louisiana State University, and the U.S. Air Force Office of Scientific Research under Contract Number FA9550-06-1-0121. 


\section{Table of Contents}

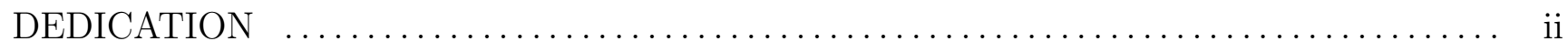

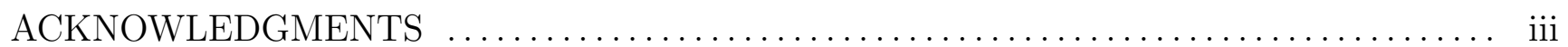

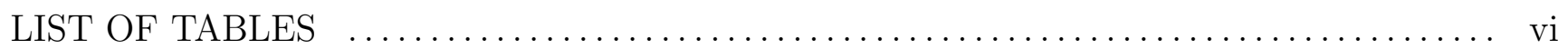

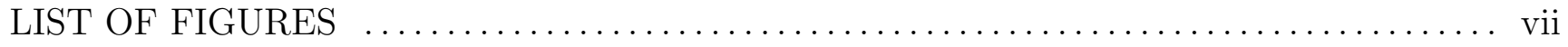

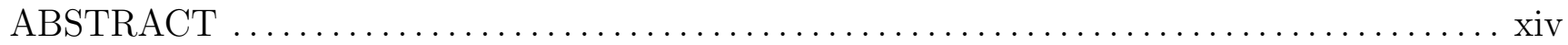

CHAPTER

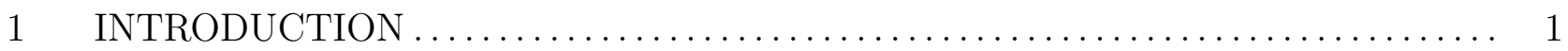

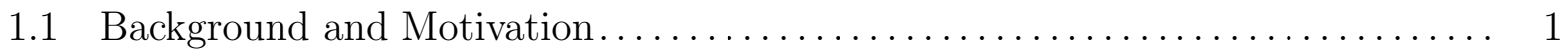

1.2 Problem Description ......................................... 6

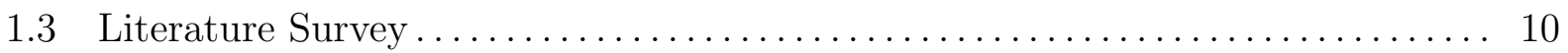

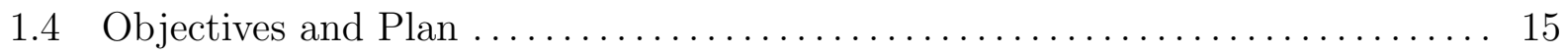

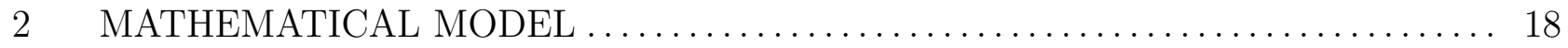

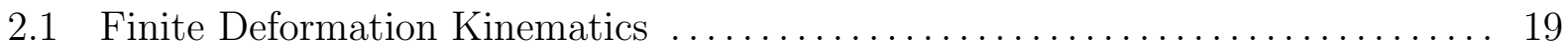

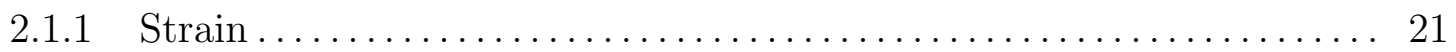

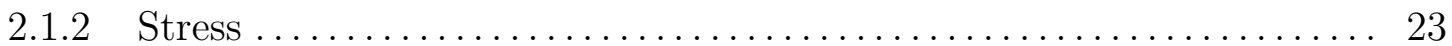

2.2 Hyperelastic Formulation of Elastic-Viscoplasticity $\ldots \ldots \ldots \ldots \ldots \ldots \ldots \ldots \ldots$

2.2 .1 Isotropic Material Behavior ............................. 26

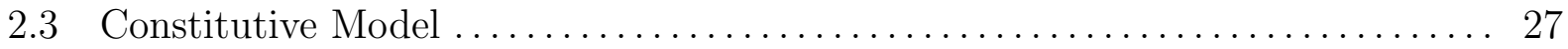

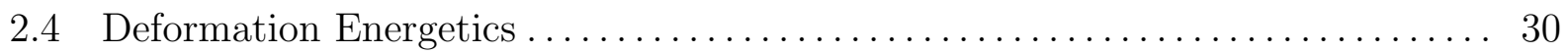

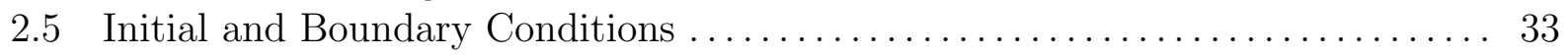

2.5.1 Contact Boundary Conditions $\ldots \ldots \ldots \ldots \ldots \ldots \ldots \ldots \ldots \ldots \ldots \ldots \ldots \ldots$

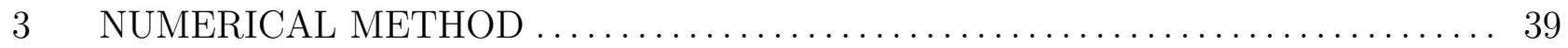

3.1 Discretization of the Governing Equations ........................ 40

3.1.1 Weak Form of the Momentum Equation..................... 42

3.1.2 Weak Form of the Temperature Equation .................... 46

3.2 Discretized Momentum Equation................................. 49

3.2 .1 Internal Force Matrix ................................. 49

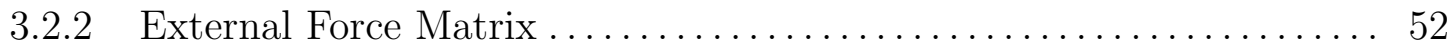

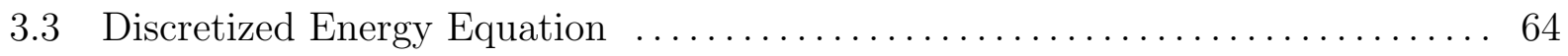

4 VERIFICATION OF THE NUMERICAL METHOD $\ldots \ldots \ldots \ldots \ldots \ldots \ldots \ldots \ldots \ldots$

4.1 Verification: Kinematic Contact Constraints ......................... 68

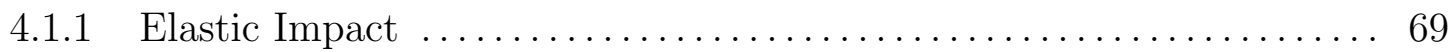

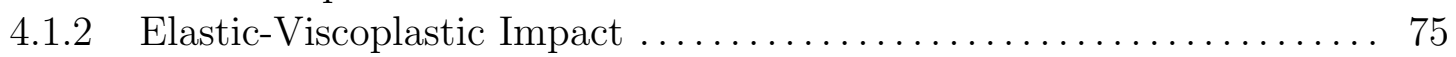

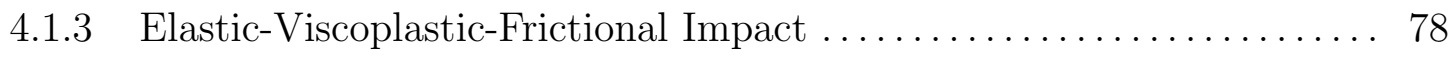

4.1.4 Sensitivity to Penalty Parameter ........................... 84

4.2 Verification: Thermal Contact Constraints ......................... 86 


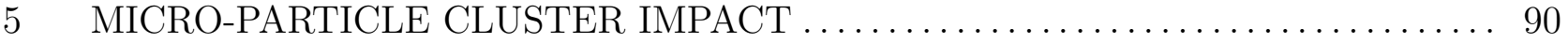

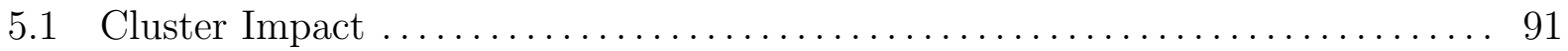

5.1 .1 Cluster Energetics .................................... 94

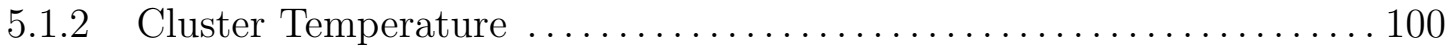

5.2 Sensitivity to Initial Cluster Configuration $\ldots \ldots \ldots \ldots \ldots \ldots \ldots \ldots \ldots \ldots \ldots \ldots$

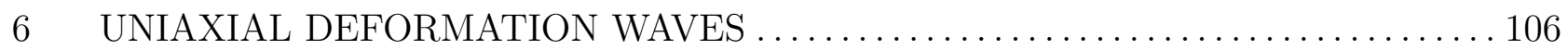

6.1 Ensemble Generation and Post-Processing .......................... 106

6.1.1 Ensemble Configuration ................................ 106

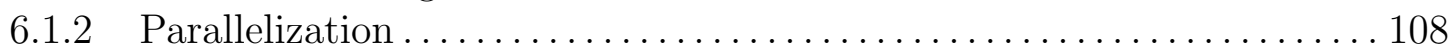

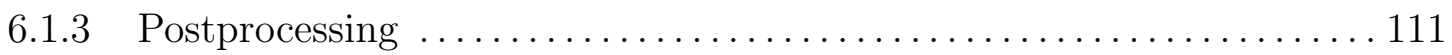

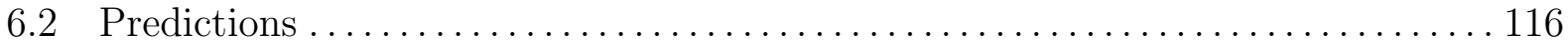

6.2.1 Mechanical Response .................................. 116

6.2.2 Thermal Response..................................... 133

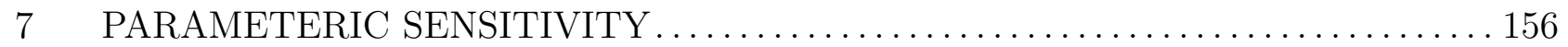

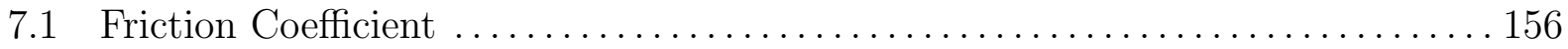

7.1.1 Mechanical Response .................................... 157

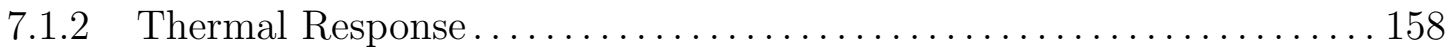

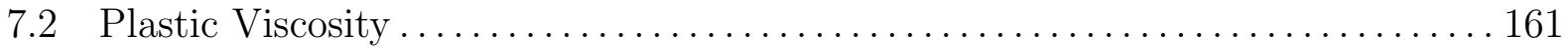

7.2.1 Mechanical Response .................................... 162

$7.2 .2 \quad$ Thermal Response.................................... 164

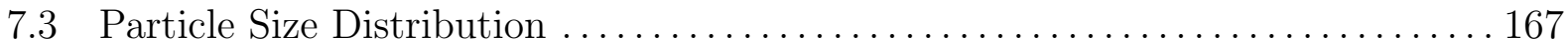

7.3.1 Mechanical Response .................................. 169

7.3.2 Thermal Response.................................. 171

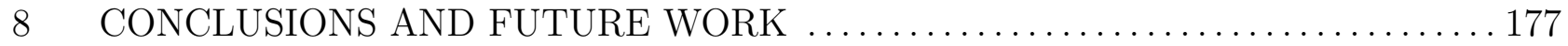

8.1 Micro-Particle Cluster Impact.................................. 177

8.2 Uniaxial Deformation Waves ..................................... 179

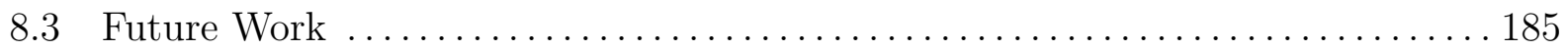

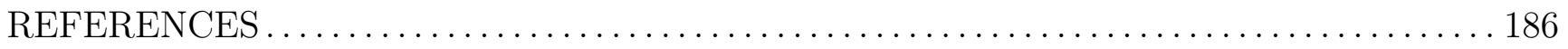

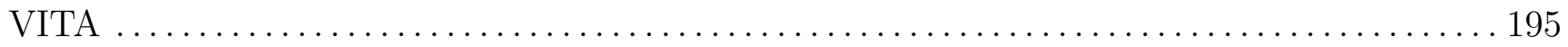




\section{List of Tables}

4.1 Material properties and numerical parameters used in this study. $\ldots \ldots \ldots \ldots \ldots$

4.2 Cases presented for verification of elastic, elastic-viscoplastic and elastic-

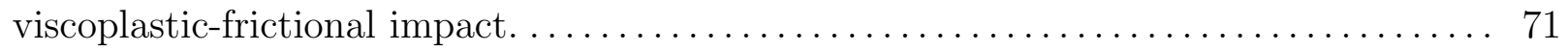

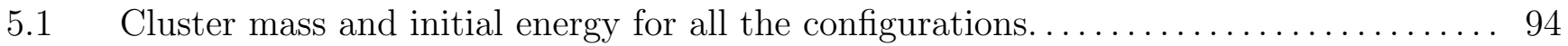

5.2 Partition of total energy and numerical error for cluster Configuration $1 \ldots \ldots \ldots \ldots 9$

5.3 Partition of total energy and numerical error for cluster Configuration $1 \ldots \ldots \ldots . \ldots 9$ 


\section{List of Figures}

1.1 Representative meso-structure of an HMX-based explosive $[116] \ldots \ldots \ldots \ldots \ldots \ldots 2$

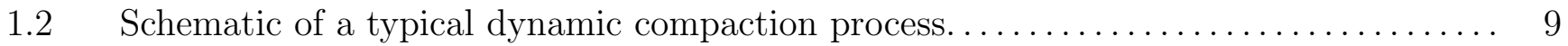

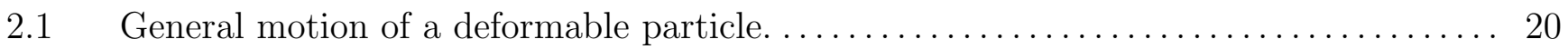

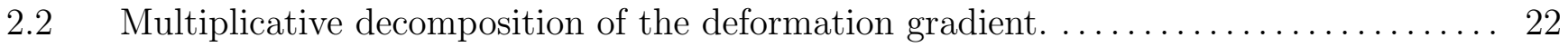

2.3 Boundary conditions of the two particle contact problem on the (a)

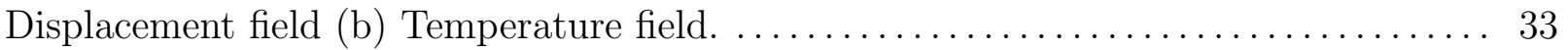

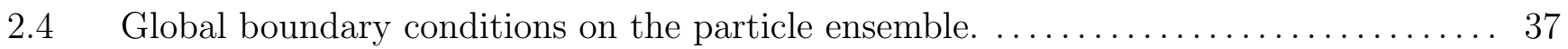

3.1 Discretization of a representative particle $\Omega_{0} \ldots \ldots \ldots \ldots \ldots \ldots \ldots \ldots \ldots \ldots \ldots$

3.2 Incremental motion of a finite element between time $t_{n-1}$ and $t_{n} \ldots \ldots \ldots \ldots \ldots$

3.3 Schematic of the discrete element contact model: (a) interaction of target and contactor discrete elements; (b) interaction of target and

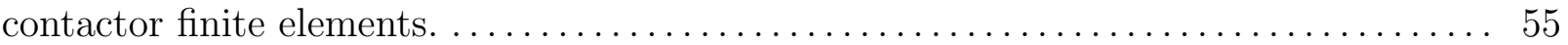

3.4 Schematic of the finite element contact model: contactor and target

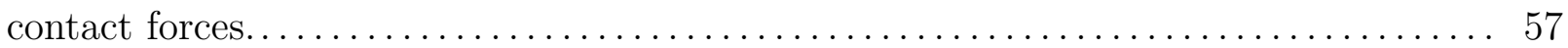

3.5 (a) Classical friction law (b) Regularized friction law. ................... 59

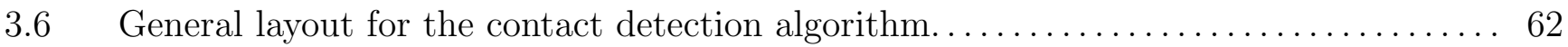

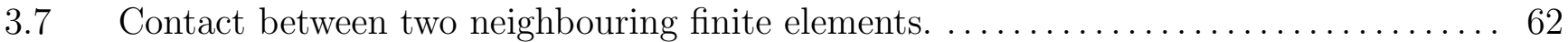

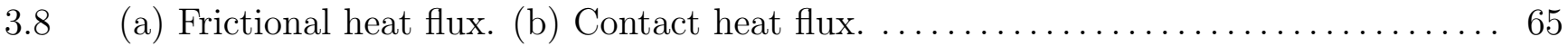

4.1 Transverse impact of two identical right circular cylinders in plane

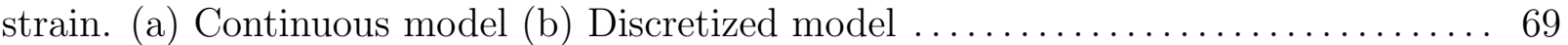

4.2 Energy history profiles for elastic impact of right circular cylinders. . . . . . . . . 72

4.3 History profiles of (a) Compressive Load and (b) Radial Compression for elastic impact of right circular cylinders....................... 74

4.4 Variation of (a) maximum radial compression and (b) maximum com-

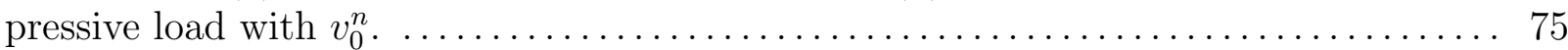

4.5 Spatial and temporal evolution of plastic work per unit mass resulting from elastic-viscoplastic impact at $v_{0}^{n}=500 \mathrm{~m} / \mathrm{s} \ldots \ldots \ldots \ldots \ldots \ldots \ldots \ldots \ldots \ldots$ 
4.6 Energy history profiles for elastic-viscoplastic impact of right circular cylinders. . . . 77

4.7 (a) Variation in coefficient of restitution with $v_{0}^{n}$ for elastic-viscoplastic impact . . . 78

4.8 Spatial contours of pressure and in-plane shear stress for Case $\mathbf{P}_{4}$ (a) Ls-Dyna and (b) FDEM. All values shown in the color-bar are in GPa. ........... 79

4.9 Energy history profiles for elastic-viscoplastic-frictional impact of right

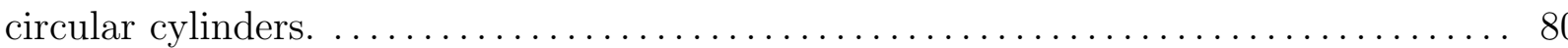

4.10 Spatial contours of in-plane shear stress : (a) Ls-Dyna and (b) FDEM. All values shown in the color-bar are in GPa. ...................... 82

4.11 Variation in frictional dissipation with respect to (a) impact angle and (b) friction coefficient.

4.12 Convergence study for (a) elastic-viscoplastic impact and (b) elasticviscoplastic-frictional impact.

4.13 Sensitivity to the normal penalty parameter: (a) Variation in plastic and friction work. (b) Variation in distance separating the center of masses of the two bodies at fixed time.

4.14 Sensitivity to the tangential penalty parameter: (a) Variation in plastic and friction work. (b) Variation in distance separating the center of

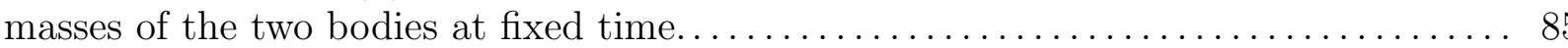

4.15 Initial configuration for thermal contact of rectangular blocks (a) Continuous model. (b) Discretized model. ...................................

4.16 (a) Temporal variation of the maximum and minimum temperatures within the blocks: Analytical and numerical predictions. (b) Spatial variation of temperature after $500 \mathrm{~ns}, 1000 \mathrm{~ns}$ and $1500 \mathrm{~ns}$ : Analytical and numerical predictions.

4.17 (a) Convergence study for thermal contact problem. (b) Sensitivity to the thermal penalty parameter: Variation of the temporal evolution of the maximum temperatures within the blocks.

5.1 Schematic of a (a) moving impactor and stationary energetic solid and (b) stationary wall and moving energetic solid.

5.2 Initial particle packing configurations used in this study: (a) Configuration 1, (b) Configuration 2, (c) Configuration 3, and (d) Configuration

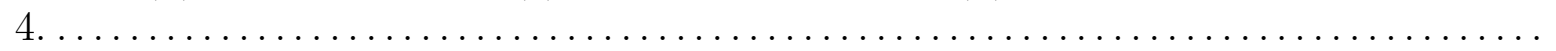

5.3 Predicted particle positions and kinetic energy contours for case $\mathbf{F} .0^{\circ}$. 93 
$5.4 \quad$ Predictions for the partitioning of total cluster energy. $\ldots \ldots \ldots \ldots \ldots \ldots \ldots \ldots \ldots . . \ldots 5$

5.5 Predictions for the temporal and spatial variation in average specific (a) plastic work and (b) friction work. Here, $x$ is normal distance from the wall.

5.6 Predicted temperature contours within particles at $t=300 \mathrm{~ns}$. All values shown in the colorbars are in Kelvin. ........................... 101

$5.7 \quad$ Predictions for distribution of hot-spot mass fractions. $\ldots \ldots \ldots \ldots \ldots \ldots \ldots \ldots \ldots \ldots \ldots$

5.8 Predicted variation in (a) plastic work (b) friction work with impact

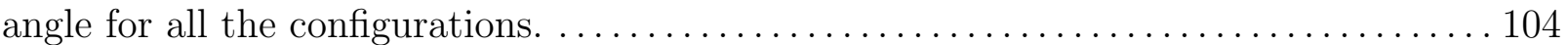

5.9 Predicted temperature contours within particles at $t=300 \mathrm{~ns}$. All values shown in the colorbars are in Kelvin............................. 105

6.1 Schematic of the initial system configuration used in this study. . . . . . . . . . ... 107

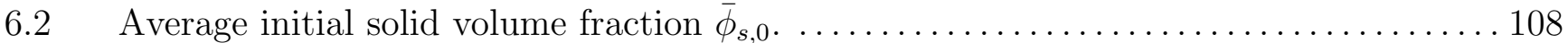

6.3 Schematic of the initial system configuration used in this study. . . . . . . . . . . . 109

$6.4 \quad$ Scaling curve for the parallel FORTRAN code $\ldots \ldots \ldots \ldots \ldots \ldots \ldots \ldots \ldots \ldots \ldots \ldots \ldots$

6.5 Schematic of the transverse averaging procedure used to obtain 1D profiles......... 112

6.6 Schematic of the discretization procedure used to obtain hot-spot contour plots.

6.7 Predictions for the average solid volume fraction $\bar{\phi}_{s}$ at $2.75 \mu \mathrm{s}$ for (a)

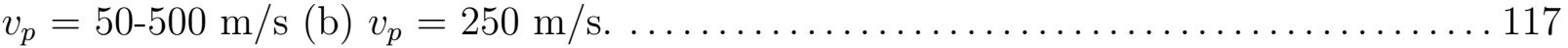

6.8 Predicted particle positions for $v_{p}=50,250$ and $500 \mathrm{~m} / \mathrm{s}$ at $1.5 \mu \mathrm{s} \ldots \ldots \ldots \ldots \ldots$

6.9 Predictions for the temporal variation of the average axial velocity $\bar{v}_{x}$ for (a) $50 \mathrm{~m} / \mathrm{s}$ and (b) $500 \mathrm{~m} / \mathrm{s}$ case. Profiles are shown in time-steps of $0.25 \mu \mathrm{s} . \ldots 121$

6.10 (a) Predictions for the temporal variation of the RMS fluctuation in axial velocity $\widetilde{v}_{x}$ for $v_{p}=50$ and $500 \mathrm{~m} / \mathrm{s}$. Profiles are shown in timesteps of $0.25 \mu \mathrm{s}$. (b) Predictions for the RMS fluctuation in axial velocity $\widetilde{v}_{x}$ at $2.75 \mu \mathrm{s}$ for $v_{p}=50$ and $500 \mathrm{~m} / \mathrm{s}$

6.11 Predictions for the RMS fluctuation in transverse velocity $\widetilde{v}_{y}$ at $2.75 \mu \mathrm{s}$ for $v_{p}=50$ and $500 \mathrm{~m} / \mathrm{s}$. 
6.12 (a) Predictions for the temporal variation of the average pressure $\bar{p}$ for $v_{p}=50,250$ and $500 \mathrm{~m} / \mathrm{s}$. Profiles are shown in time-steps of $0.25 \mu \mathrm{s}$.

(b) Predictions for the average pressure $\bar{p}$ at $2.75 \mu \mathrm{s}$ for $v_{p}=50,250$ and $500 \mathrm{~m} / \mathrm{s} . \ldots 124$

6.13 Predicted particle averaged pressure contours at $2.75 \mu \mathrm{s}$ for $v_{p}=50$, 250 and $500 \mathrm{~m} / \mathrm{s}$. Values shown in the color-bar are in GPa................... 125

6.14 Predictions for the average (a) Cauchy stress components $\overline{\boldsymbol{\sigma}}$ and (b) deviatoric stress components $\overline{\overline{\boldsymbol{\sigma}}}$ at $2.75 \mu \mathrm{s}$ for $v_{p}=50,250$ and $500 \mathrm{~m} / \mathrm{s} \ldots \ldots \ldots \ldots 127$

6.15 (a) Predictions for the temporal variation of the average Von Mises stress $\overline{\boldsymbol{\tau}}_{e}$ for $v_{p}=50$ and $500 \mathrm{~m} / \mathrm{s}$. Profiles are shown in time-steps of $0.25 \mu \mathrm{s}$. (b) Predictions for the average Von Mises stress $\overline{\boldsymbol{\tau}}_{e}$ at $2.75 \mu \mathrm{s}$

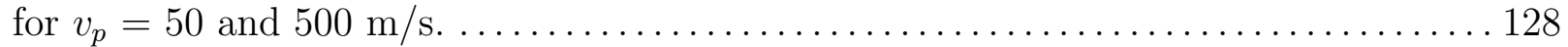

6.16 Predictions for the average effective plastic strain $\bar{\epsilon}_{p}$ at $2.75 \mu \mathrm{s}$ for (a) $v_{p}=50-500 \mathrm{~m} / \mathrm{s}(\mathrm{b}) v_{p}=250 \mathrm{~m} / \mathrm{s}$.

6.17 Predicted finite element contours of Von Mises stress $\boldsymbol{\tau}_{e}$ within particles at $2.75 \mu \mathrm{s}$ for $v_{p}=50,250$ and $500 \mathrm{~m} / \mathrm{s}$. Values shown in the color-bars are in $\mathrm{GPa}$

6.18 Magnified portion of the finite element contour of Von Mises stress $\boldsymbol{\tau}_{e}$ within particles at $2.75 \mu \mathrm{s}$ for $v_{p}=50 \mathrm{~m} / \mathrm{s}$.

6.19 (a) Predicted wave speed for $v_{p}=250 \mathrm{~m} / \mathrm{s}$. (b) Predicted variation in precursor $\left(D_{l}\right)$ and plastic $\left(D_{t}\right)$ wave speed with piston speed $\left(v_{p}\right)$.

6.20 Hugoniot for (a) wave-piston speed and (b) pressure-piston speed. (1) Numerical predictions from the FDEM (2) Experimental data from ref. [121]. (3) Experimental data from ref. [92]. (4) Experimental data from ref. [121].

6.21 Comparison of the Hugoniot for (a) wave-piston speed and (b) pressurepiston speed between the 2000 and 4000 particle ensemble.

6.22 Predictions for the (a) plastic mass-fraction $\bar{m}_{p}$ and (b) friction mass fraction $\bar{m}_{f}$ at $2.75 \mu \mathrm{s}$ for $v_{p}=50-500 \mathrm{~m} / \mathrm{s}$.

6.23 Predictions for the partitioning of (a) total system energy and (b) massspecific system energy at $2.75 \mu$ s for $v_{p}=50,250$ and $500 \mathrm{~m} / \mathrm{s} \ldots \ldots \ldots \ldots \ldots \ldots$

6.24 Predictions for the temporal variation of average (a) plastic heating rate $\overline{\dot{w}}_{p}$ and (b) friction heating rate $\overline{\dot{w}}_{f}$ for $v_{p}=50,250$ and $500 \mathrm{~m} / \mathrm{s}$. Profiles are shown in time-steps of $0.25 \mu \mathrm{s}$ 
6.25 Predictions for the (a) average temperature $\bar{T}$ for $v_{p}=50-500 \mathrm{~m} / \mathrm{s}$, (b) maximum temperature $T_{\max }$ for $v_{p}=50,250$ and $500 \mathrm{~m} / \mathrm{s}$ and (c) variation of average temperature $\bar{T}$ with average effective plastic strain

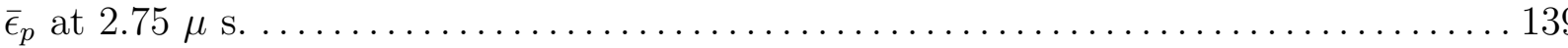

6.26 Predicted particle averaged temperature contours at $2.75 \mu \mathrm{s}$ for $v_{p}=$ 50,250 and $500 \mathrm{~m} / \mathrm{s}$. All values shown in the color-bar are in Kelvin.

6.27 Predicted finite element temperature contour at $2.75 \mu \mathrm{s}$ for $v_{p}=$ $500 \mathrm{~m} / \mathrm{s}$. All values shown in the color-bar are in Kelvin. ..

6.28 Predicted finite element adiabatic plastic temperature contour at $2.75 \mu \mathrm{s}$ for $v_{p}=500 \mathrm{~m} / \mathrm{s}$. All values shown in the color-bar are in Kelvin.

6.29 Predicted temporal variation of hot-spot mass-fraction for $v_{p}=50 \mathrm{~m} / \mathrm{s}$. Values in the color-bar represent the logarithm of mass-fraction.

6.30 Predicted temporal variation of hot-spot mass-fraction for $v_{p}=250 \mathrm{~m} / \mathrm{s}$. Values in the color-bar represent the logarithm of mass-fraction. . . . . . . . . . . 146

6.31 Predicted temporal variation of hot-spot mass-fraction for $v_{p}=500 \mathrm{~m} / \mathrm{s}$. Values in the color-bar represent the logarithm of mass-fraction.

6.32 Predicted hot-spot mass-fraction contours based on temperature rise estimated using (a) energy analysis and (b) adiabatic plasticity theory at $2.75 \mu \mathrm{s}$ for $v_{p}=50,250$ and $500 \mathrm{~m} / \mathrm{s}$. Values in the color-bar represent the logarithm of mass-fraction.

6.33 Predicted spatial variation of hot-spot mass-fraction through the compaction wave at $2.75 \mu$ s for $v_{p}=50,250$ and $500 \mathrm{~m} / \mathrm{s} \ldots \ldots \ldots \ldots \ldots \ldots \ldots \ldots \ldots \ldots \ldots \ldots \ldots$

6.34 Variation of time to thermal explosion with hot-spot temperature . . . . . . . . . . 152

6.35 (a) Gamma distribution function. (b) Hot-spot distribution for semi-

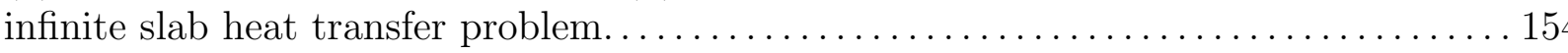

7.1 Predictions for the (a) average solid volume fraction $\bar{\phi}_{s}$ and (b) RMS fluctuation in axial velocity $\widetilde{v}_{x}$ at $2.75 \mu \mathrm{s}$ for $v_{p}=250 \mathrm{~m} / \mathrm{s}$ for $\mu=0.0$, $0.05,0.15$ and 0.25 .

7.2 Predictions for the (a) average Von Mises stress $\overline{\boldsymbol{\tau}}_{e}$ and (b) average effective plastic strain $\bar{\epsilon}_{p}$ at $2.75 \mu \mathrm{s}$ for $v_{p}=250 \mathrm{~m} / \mathrm{s}$ for $\mu=0.0,0.05$, 0.15 and 0.25

7.3 Predicted variation in (a) precursor $\left(D_{l}\right)$ and (b) plastic $\left(D_{t}\right)$ wave speed with piston speed $\left(v_{p}\right)$ for $\mu=0.0,0.05,0.15$ and 0.25 . 
7.4 Predictions for the (a) average temperature $\bar{T}$ and (b) maximum temperature $T_{\max }$ for $v_{p}=250 \mathrm{~m} / \mathrm{s}$ at $2.75 \mu \mathrm{s}$ for $\mu=0.0,0.05,0.15$ and 0.25 .

7.5 Predicted hot-spot mass-fraction contours for $v_{p}=250 \mathrm{~m} / \mathrm{s}$ at $2.75 \mu \mathrm{s}$ for $\mu=0.0,0.05,0.15$ and 0.25 . Values in the color-bar represent the logarithm of mass-fraction.

7.6 Predicted variation of hot-spot mass-fraction behind the compaction

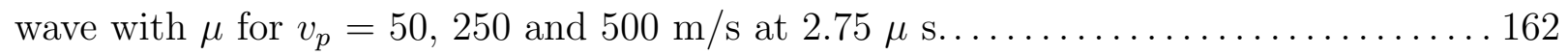

7.7 Predictions for the (a) average solid volume fraction $\bar{\phi}_{s}$ and (b) RMS fluctuation in axial velocity $\widetilde{v}_{x}$ at $2.75 \mu \mathrm{s}$ for $v_{p}=250 \mathrm{~m} / \mathrm{s}$ for $\nu=10$

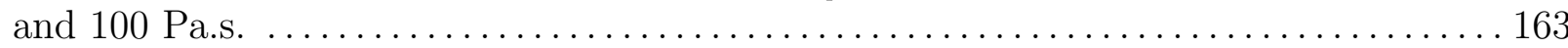

7.8 Predictions for the (a) average Von Mises stress $\overline{\boldsymbol{\tau}}_{e}$ and (b) average effective plastic strain $\bar{\epsilon}_{p}$ at $2.75 \mu \mathrm{s}$ for $v_{p}=250 \mathrm{~m} / \mathrm{s}$ for $\nu=10$ and 100 Pa.s..... 164

7.9 Predicted variation in (a) precursor $\left(D_{l}\right)$ and (b) plastic $\left(D_{t}\right)$ wave

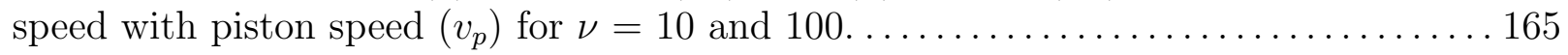

7.10 Predictions for the (a) average temperature $\bar{T}$ and (b) maximum temperature $T_{\max }$ for $v_{p}=250 \mathrm{~m} / \mathrm{s}$ at $2.75 \mu \mathrm{s}$ for $\nu=10$ and 100 Pa.s............ 165

7.11 Predicted hot-spot mass-fraction contours for $v_{p}=500 \mathrm{~m} / \mathrm{s}$ at $2.75 \mu \mathrm{s}$ for $\nu=10$ and 100 Pa.s. Values in the color-bar represent the logarithm of mass-fraction.

7.12 Predicted variation of hot-spot mass-fraction behind the compaction wave with $\nu$ for $v_{p}=50,250$ and $500 \mathrm{~m} / \mathrm{s}$ at $2.75 \mu \mathrm{s} \ldots \ldots \ldots \ldots \ldots \ldots \ldots \ldots \ldots \ldots \ldots$

7.13 Schematic of the alternate initial system configuration used in this study. ........ 168

7.14 Initial particle size distribution for (a) Configuration S-1 and (b) Con-

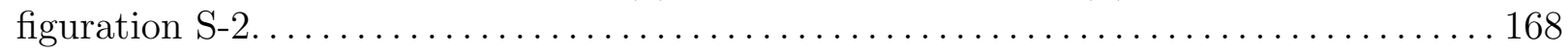

7.15 Average initial solid volume fraction $\bar{\phi}_{s, 0}$ for configuration S-1 and S-2. ........ 169

7.16 Predictions for the (a) average solid volume fraction $\bar{\phi}_{s}$ and (b) average effective plastic strain $\bar{\epsilon}_{p}$ at $2.75 \mu \mathrm{s}$ for $v_{p}=250 \mathrm{~m} / \mathrm{s}$ for configuration

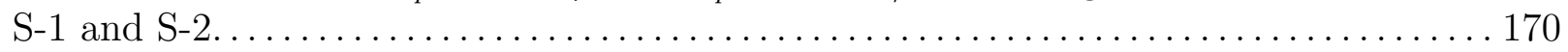

7.17 Predictions for the average pressure $\bar{p}$ for all particle size classes at $2.75 \mu \mathrm{s}$ for $v_{p}=250 \mathrm{~m} / \mathrm{s}$ for configuration S-1 and $\mathrm{S}-2$.

7.18 Predicted variation in (a) precursor $\left(D_{l}\right)$ and $(\mathrm{b})$ plastic $\left(D_{t}\right)$ wave speed with piston speed $\left(v_{p}\right)$ for configuration S-1 and S-2. 
7.19 Predictions for the (a) average temperature $\bar{T}$ and (b) maximum temperature $T_{\max }$ for $v_{p}=250 \mathrm{~m} / \mathrm{s}$ at $2.75 \mu \mathrm{s}$ for configuration S-1 and

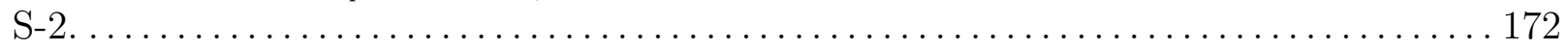

7.20 Predictions for the average temperature $\bar{T}$ for all particle size classes at $2.75 \mu \mathrm{s}$ for $v_{p}=250 \mathrm{~m} / \mathrm{s}$ for configuration S-1 and S-2.

7.21 Predicted hot-spot mass-fraction contours for $v_{p}=50,250$ and $500 \mathrm{~m} / \mathrm{s}$ at $2.75 \mu \mathrm{s}$ for configuration S-1 and S-2. Values in the color-bar represent the logarithm of mass-fraction.

7.22 Predicted variation of hot-spot mass-fraction behind the compaction wave with particle size distribution for $v_{p}=50,250$ and $500 \mathrm{~m} / \mathrm{s}$ at $2.75 \mu \mathrm{s}$.

7.23 Predicted variation of hot-spot mass-fraction behind the compaction wave for each particle size class for $v_{p}=250 \mathrm{~m} / \mathrm{s}$ at $2.75 \mu \mathrm{s}$ for configuration S-1 and S-2 


\section{Abstract}

An explicit, two-dimensional, Lagrangian finite and discrete element technique is formulated and used to computationally characterize meso-scale fluctuations in thermomechanical fields induced by low pressure deformation waves propagating through particulate energetic solids. Emphasis is placed on characterizing the relative importance of plastic and friction work as meso-scale heating mechanisms which may cause bulk ignition of these materials and their dependence on piston speed $\left(v_{p} \sim 50-500 \mathrm{~m} / \mathrm{s}\right)$. The numerical technique combines conservation principles with a plane strain, thermoelastic-viscoplastic constitutive theory to describe deformation within the material meso-structure. An energy consistent, penalty based, distributed potential force method, coupled to a penalty regularized Amontons Coulomb law, is used to enforce kinematic and thermal contact constraints between particles. The technique is shown to be convergent, and its spatial $(\sim 2.0)$ and temporal $(\sim 1.5)$ convergence rate is established. Predictions show that alhough plastic work far exceeds friction work, considerably higher local temperatures result from friction work. Most mass within the deformation wave $(\sim 99.9 \%)$ is heated to approximately 330, 400, and $500 \mathrm{~K}$, for $v_{p}=50,250$, and $500 \mathrm{~m} / \mathrm{s}$, respectively, due to plastic work, whereas only a small fraction of mass $\left(\sim 10^{-3 \%}\right)$ is respectively heated to temperatures in excess of 600,1100 and $1400 \mathrm{~K}$ due to friction work. In addition to low speed impact, and contrary to conventional belief, friction work is shown to also be an important heating mechanism at higher impact speeds. The variation in spatial partitioning of bulk energy within the deformation wave structure with particle morphology and material properties is demonstrated. 


\section{Chapter 1}

\section{Introduction}

This study addresses the theory and meso-scale modeling of deformation waves in particulate energetic solids. Here, meso-scale refers to length scales over which interactions between discrete particles are important. The main focus of this study is to characterize deformation induced fluctuations occurring in mechanical and thermodynamic fields which arise due to the heterogeneous structure of these materials. Unlike inert solids, these fluctuations can cause the onset of particle scale combustion which can significantly affect the bulk (or engineering) scale response of energetic solids. In this chapter, background information and motivation for this study are first discussed, followed by a brief description of the physical model, and a survey of relevant experimental and modeling literature. Then, specific objectives of this study are outlined, and the plan of this dissertation is given.

\subsection{Background and Motivation}

Energetic solids, such as propellants, pyrotechnics, and explosives, are routinely used as selfcontained energy sources for numerous commercial, aerospace, and defense applications including automobile airbag inflators, spacecraft thrusters, and weapons. These solids typically consists of mixtures of metal and/or explosive particles that are consolidated with a small amount of plasticlike binder material. Individual particles can vary in size from approximately $10 \mathrm{~nm}$ to $250 \mu \mathrm{m}$, and can vary in shape from rod-like to almost spherical. Examples of common solid high-explosives, which are the focus of this study, include RDX $\left(\mathrm{C}_{3} \mathrm{H}_{6} \mathrm{~N}_{6} \mathrm{O}_{6}\right)$, HMX $\left(\mathrm{C}_{4} \mathrm{H}_{8} \mathrm{~N}_{8} \mathrm{O}_{8}\right)$, and PETN $\left(\mathrm{C}_{5} \mathrm{H}_{8} \mathrm{~N}_{4} \mathrm{O}_{12}\right)$; the heterogeneous meso-structure of a representative HMX-based, plastic-bonded explosive is shown in Fig. 1.1 [116]. When burned, these materials produce copious amounts of high-temperature gas that is capable of performing significant work $(\approx 8.1-9.5 \mathrm{MJ} / \mathrm{kg}[75,108])$. The combustion rate, which determines power output, depends on many factors including the 
cause of ignition (i.e., mechanical or thermal stimulus), extent of confinement, and material mesostructure. The material meso-structure is characterized by such features as particle size and shape distributions, porosity, and intra-particle defects and impurities.

Deflagration refers to low speed, low pressure combustion (0.4-3 Km/s and 0.1-1 GPa), whereas detonation refers to high speed, high pressure combustion (6-8 km/s and 10-100 GPa). Of particular importance is the process where deflagration transitions to detonation, commonly referred to as Deflagration-to-Detonation Transition (DDT) $[48,90]$. This process can often be accidentally triggered by reasonably mild impact (impact speeds 50-100 m/s), causing great concern over the safe handling, transportation, and storage of these materials. As such, a fundamental understanding of impact induced heating and ignition of energetic solids is necessary for their continued development, and for their performance and safety assessment.

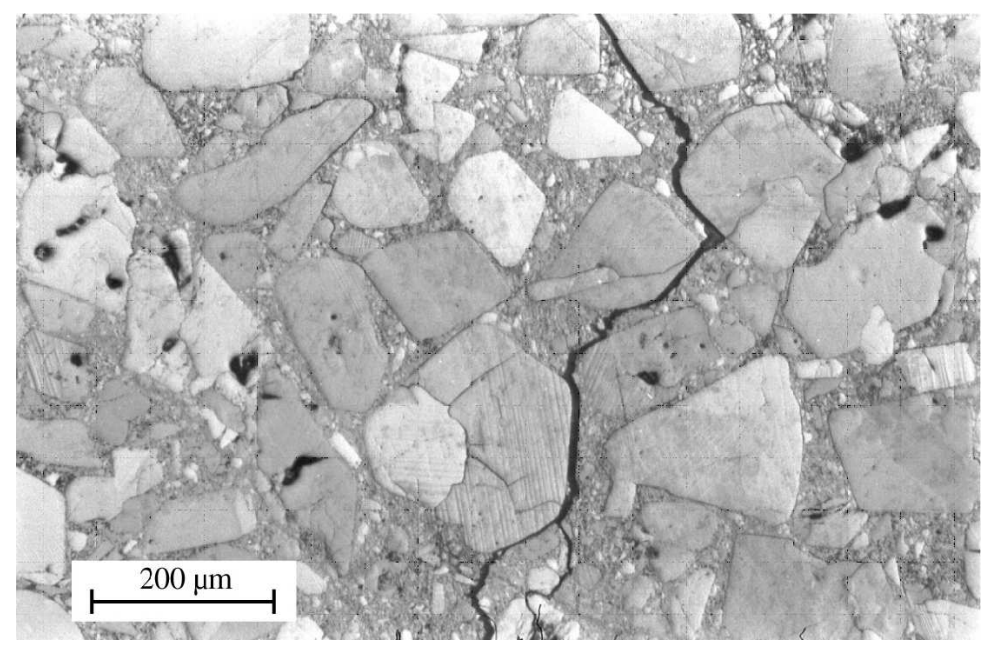

Figure 1.1: Representative meso-structure of an HMX-based explosive [116].

Unlike homogeneous solids, heterogeneous solids transmit applied bulk loads by inter-particle contact which can lead to strain and stress localization within particles in the vicinity of contact surfaces, even for mild impact. Associated localization of thermal energy within and between particles by dissipative mechanisms can produce small mass regions of elevated temperature, referred to as hot-spots, that may locally ignite the material. Hot-spots are typically sub-particle scale in size (0.1-10 $\mu \mathrm{m}[44])$, and may have temperatures in excess of $600 \mathrm{~K}$ lasting for several microseconds, provided that they possess sufficient thermal inertia to overcome conductive losses. 
Neighboring hot-spots may thermally interact, collectively resulting in bulk ignition of the material by the impact induced deformation wave. Exothermic energy release by reacting hot-spots to form high-temperature, high-pressure gas intensifies acoustic transmission of energy through the material, strengthening bulk deformation wave propagation, and possibly causing transition to detonation by DDT. As such, it is well-accepted that reactive hot-spot formation at the particle scale plays a central role in determining impact sensitivity of energetic solids. However, characterizing the physical mechanisms responsible for hot-spot formation, and their dependence on impact conditions, meso-structure, and particle scale thermomechanical properties, remains an unresolved issue.

Bulk DDT models applied to engineering scale systems in practice, which are typically based on continuum mixture field theories $[66,132,114]$, are formulated in terms of spatially averaged thermodynamic state variables; consequently, they provide no information about local fluctuations in these variables that are important for combustion initiation. This model deficiency has motivated the development of complex mechanistic sub-models to implicitly account for the effect of hot-spots within the context of bulk models [31, 65, 68, 89, 4, 48]. Though these highly refined bulk models can predict most aggregate features of DDT, their accuracy is usually limited to a narrow range of impact conditions about which the sub-models have been correlated to data; a comprehensive, critical examination of the most commonly used two-phase, mixture theories for DDT in granular explosives can be found in Ref. [13]. Moreover, DDT experiments, while useful, also typically provide only aggregate information about DDT, such as bulk wave speeds and pressures. No robust experimental diagnostic techniques currently exist that can provide resolved information about local thermodynamic fields occurring within particles during impact. In the absence of such data, it is common for modelers to infer hot-spot information based on the implications of bulk measurements. Direct meso-scale modeling of particulate solids can provide both qualitative and quantitative information concerning the relative importance of hot-spot formation mechanisms. This information can then be used to facilitate the rational development of improved, hot-spot motivated, bulk burn sub-models for use in applications. 
Potential hot-spot mechanisms include particle fracture [38], inter-particle friction [35, 41], plastic deformation of particles $[7,22,35,46,51,73]$, jetting and viscous heating of particle interfaces $[46,89,97,135]$, and adiabatic gas compression by interstitial void collapse [27, 34]. Which mechanism, or collection of mechanisms, dominates strongly depends on the impact conditions and material meso-structure. Of these mechanisms, friction is probably the least well characterized, and arguably the most important, particularly for mild impact which is most representative of an accidental impact scenario. Computational evidence suggests that hot-spots formed by strong, high pressure impact (impact speed $\sim 1000 \mathrm{~m} / \mathrm{s}$, pressure $\sim 5 \mathrm{GPa}$ ) of metals and explosives are primarily due to highly localized inelastic void collapse [46, 65, 68, 91]; fracture and friction are believed to be of secondary importance.

Dissipative mechanisms applicable to pore collapse by strong shocks are viscous heating and plastic work due to the intense stresses involved which may significantly exceed the material yield strength resulting in "fluid-like" behavior. Indeed, early hydrodynamic simulations of heterogeneous initiation by Mader [91] showed that material micro-jets formed when a strong shock impinges on small interstitial pores can produce hot-spots as the micro-jets impact material located on the opposite side of the pores. Bowden and Yoffe [27] were the first to show that adiabatic compression of gas trapped within rapidly deforming material can result in significant heating and may lead to ignition. Further, Chaudhri and Field [34] showed that trapped gas compressed by even relatively low pressure shocks (0.1 GPa) might ignite sensitive explosives. Frey [46], Grady [51], and Kipp [73] developed a two-step mechanistic model to describe viscoplastic heating within thin shear bands located around heterogeneities; they argued that hot-spots are produced by plastic work which causes material to melt, followed by intense viscous heating within the resulting fluid. For mild, low pressure impact (impact speed $\sim 100 \mathrm{~m} / \mathrm{s}$, pressure $\sim 0.8 \mathrm{GPa}$ ), it is generally accepted that plastic deformation of the meso-structure remains a dominant hot-spot formation mechanism [7]; again, inter-particle friction has mostly been ignored. Conley and Benson [37] argued that frictional dissipation is of secondary importance to plastic work in the absence of large material deformation. However, both theoretical [1] and experimental [25, 27, 36] studies 
have shown that ignition first occurs from thermal decomposition of explosive along frictional boundaries. In fact, experimental studies performed by Bowden [26] provide strong evidence that initiation by mild impact originates at frictional surfaces due to hot-spots. Also, Kumar and Kumar [76] predicted frictionally induced temperatures between 1604-2730 K resulting from dynamic compaction (impact speed of $500 \mathrm{~m} / \mathrm{s}$ ) of several symmetrically packed metal particles. These results collectively suggest that additional studies are needed to differentiate the relative importance of friction work and plastic work as hot-spot formation mechanisms for different loading conditions and material meso-structures.

Questions remain concerning both the bulk and meso-scale thermomechanics of deformation waves in energetic solids. Because these waves do not induce well-defined equilibrium states due to temporal and spatial fluctuations in thermomechanical fields at the meso-scale, their structure must be statistically described in terms of average and fluctuating components. In this work, wave structure refers to the spatial variation in thermomechanical variables through the wave at fixed time. Both the bulk and local partitioning of deformation wave energy into reversible and irreversible components within the wave, and how this partitioning is affected by loading rate and material meso-structure, remain unclear. In particular, characterizing thermal energy fluctuations associated with hot-spots remains a topic of significant interests because of its relevance to combustion. Though meso-scale modeling can be used to provide such information in the absence of meso-scale data, predictions depend on the details of the constitutive theory used. For example, both rate-independent and dependent constitutive theories have been used to describe the mechanics of these materials $[61,111,37]$. Meso-scale simulations performed by Menikoff, et al [98], and Conley, et al [37], predict that bulk wave propagation in granular HMX is indicative of significant viscoplasticity. However, the value of the plastic viscosity parameter used in these models, which determines the stress relaxation rate to the yield surface, is chosen to reflect data from a limited number of experiments [40]. The influence of plastic viscosity on the deformation mechanics and energetics is not well-characterized. Also, little data exist for establishing friction coefficient values for high explosives such as HMX. Dobratz [42] gives values ranging between 0.25 
to 1.1 for several explosive mixtures in sliding contact with themselves. Clearly, it is reasonable to expect that the friction coefficient may significantly influence deformation wave thermomechanics. It is, therefore, both desirable and necessary to characterize the variation in wave structure and hot-spot formation with key constitutive parameters that contain significant uncertainty.

Experiments performed by Lowe, et al $[84,85]$, indicated that resistance to volumetric deformation (i.e., compaction) of granular solids depends on particle size, with small particles exhibiting greater resistance due to enhanced stress bridging between particles. As such, it is plausible that small particles may decrease impact sensitivity by suppressing hot-spot formation, temperature, and mass-fraction. This assertion is supported by the experiments of Bowden [27], and those of Howe, et al [59], and Sheffield, et al [121], which indicated that explosives consisting of larger particles exhibit enhanced transition to detonation, implying more intense hot-spots. The critical hot-spot temperature needed for detonation was estimated by a thermal explosion theory to increase with decreasing hot-spot size (mass) due to conductive losses, with larger particles resulting in larger hot-spots [126]. In contrast, predictions by Kumar and Kumar [76] showed that hot-spot temperatures decrease from approximately $3370 \mathrm{~K}$ to $2240 \mathrm{~K}$ when particle diameter increases from $50 \mu \mathrm{m}$ to $200 \mu \mathrm{m}$. Thus, some ambiguity exists concerning the role of particle size on hotspot formation. Such unresolved issues represent substantial challenges in understanding ignition of energetic solids by impact.

\subsection{Problem Description}

The primary focus of this study is to computationally characterize the variation in temporal and spatial partitioning of energy within the meso-structure of granular energetic solids by uniaxial deformation waves resulting from mild, low pressure impact (impact speed $\sim 50-500 \mathrm{~m} / \mathrm{s}$ ); specific objectives are outlined later in this chapter. Simulating mild impact is arguably more challenging than strong impact due to the need to simultaneous resolve coupled interactions between volumetric dissipation by plastic work and surface dissipation by inter-particle friction; in fact, most simulations performed by others have ignored friction altogether [9, 22, 95]. Particular emphasis 
is placed on characterizing the fraction of solid mass that is heated to elevated temperature, referred to as the hot-spot mass fraction, for different impact speeds, constitutive descriptions, and meso-structures, because of its relevance to the development of bulk ignition models.

A comprehensive description of deformation waves at the meso-scale should account for interactions between complex physical processes such as anisotropic viscoplasticity, fracture, friction, phase change, and combustion. Realistic descriptions would also account for complex particle geometries, size distributions, packing arrangements, and intra-particle defects. Further, because the combustion rate of energetic solids is sensitive to temperature, realistic descriptions of explosive ignition would require accurate knowledge of temperature-dependent thermomechanical material properties which characterize the dissipative multiphase mechanics. Such a problem, if properly posed, would be exceedingly difficult to computationally solve over modest size domains using conventional tools such as finite element analysis (FEA).

For tractability and ease of numerical implementation, a simplified model description is used in this study that retains important features of the real problem. To this end, the soft binder material is ignored because the harder energetic particles are often the main load bearing component, particularly under compression due to particle lock-up. The analysis is restricted to two spatial dimensions (2-D), and plane-strain conditions are imposed, enabling leading-order estimates to be obtained in a computationally efficient manner. Particles are assumed to be initially circular in shape, thereby eliminating singularities occurring in the vicinity of sharp corners located along particle surfaces. Because the average contact stress at yield is considerably smaller for spherical particles than for cylindrical particles, whereas the peak stress is only marginally smaller, greater stress concentrations occur for 3-D particles. Also, the initial porosity and number of contacts per grain differs between 2-D and 3-D particle ensembles. Assuming face centered cubic packing, 2-D circular particles have a maximum porosity of $9.3 \%$ and 6 contacts per grain, whereas 3-D spherical particles have a maximum porosity of $26 \%$ and 12 contacts per grain. Consequently, dimensionality will influence meso-scale thermomechanics. 
Because this study strictly focuses on characterizing the intensity of heating processes that may lead to combustion of energetic solids by impact, the material is assumed inert; as such, a multiphase description is not necessary. Fracture is ignored, though X-ray microtomographs of compacted HMX particles indicate substantial fracture [52]. It is likely that frictional rearrangement of fractured particles within deformation waves may significantly enhance hot-spot formation; this issue, which would require sophisticated crack initiation and propagation models, is beyond the scope of this study. It is further assumed that no fluid exists within pore spaces between particles, and phase change is ignored. At standard conditions, HMX melts at $T_{m}=520 \mathrm{~K}$, and this temperature increases with pressure [95]. Because HMX is known to melt prior to the onset of combustion, the latent heat of fusion may energetically suppress reactive hot-spot formation. More importantly, phase change limits particle strength and may significantly alter the contact mechanics by multiphase lubrication.

Several other simplifications are made concerning the constitutive theory. First, though explosive crystals often exhibit anisotropic behavior [40,98], the bulk response of particle ensembles is isotropic due to random crystal orientations within a particle. This study assumes isotropy, resulting in directionally independent properties. Second, key thermal, mechanical and transport properties, such as specific heat, yield strength, and viscosity, are temperature dependent. The potential influence of these temperature dependent properties on compaction behavior has been described by Menikoff $[95,96]$. In this study, all material properties are assumed constant, where the values chosen as representative of HMX [96]. Third, a rate-dependent associative flow law with a pressure independent Von Mises yield criterion is used to model the inelastic material response of HMX particles, and hardening and softening effects are ignored. Despite numerous simplifications, this study does capture the leading-order, coupled effects of viscoplastic void collapse and inter-particle friction induced by low pressure deformation waves within large particle ensembles.

A schematic of the model problem is shown in Fig. 1.2. The 2-D computational domain encompasses a small piece of material (6.209 $\mathrm{mm}$ long and $1.2 \mathrm{~mm}$ wide) that consists of randomly packed, initially circular particles of various sizes; the initial average solid volume fraction of the 


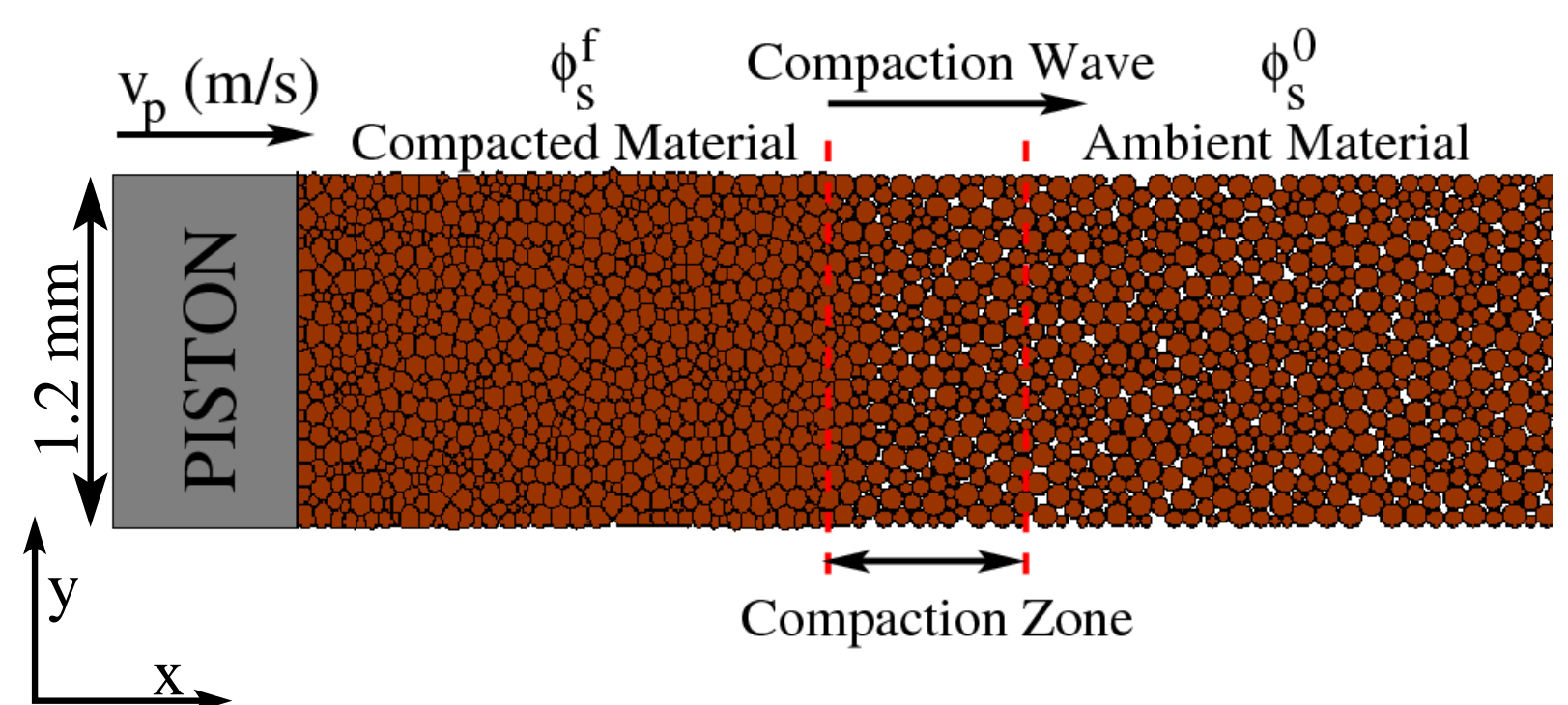

Figure 1.2: Schematic of a typical dynamic compaction process.

material is given by $\phi_{s}^{0}$ (the average initial porosity is given by $1-\phi_{s}^{0}$ ). The domain was chosen sufficiently small so that well-resolved computations could be performed in a timely manner, but sufficiently large to enable quasi-steady deformation waves (in an average sense) to evolve in response to impact. Here, uniaxial, plane strain impact by a constant velocity rigid piston $\left(v_{p}\right)$ is considered which supports the propagation of a deformation wave into the material and away from the piston. This wave is referred to as a compaction wave in this study because it results in a substantial decrease in material volume as the initial porosity is reduced. The compaction wave structure extends over a small region, referred to as the compaction zone, which connects the ambient material state to the quasi-steady final compacted state having an average solid volume fraction of $\phi_{s}^{f}>\phi_{s}^{0}$. Stress is transmitted through the compaction zone by interparticle contact resulting in plastic deformation and frictional rearrangement of particles, and the initiation and growth of hot-spots. Significant fluctuations in thermomechanical fields occur within the compaction zone. Though the compaction zone structure is complex, it generally consists of a lead precursor region where particle stress bridging is prominent, followed by a plastic region where porosity is substantially reduced. The thickness and intensity of these regions depends on impact speed. Bulk (average) quantities are obtained by spatially averaging meso-scale predictions over suitable representative elementary volumes. 


\subsection{Literature Survey}

Bulk models applied to the DDT of energetic solids describe the collective response of large particle ensembles $\left(\sim 10^{13}\right.$ particles $\left./ \mathrm{m}^{3}\right)$ [4, 64, 89, 124, 132]. These models, though essential for obtaining engineering-scale predictions over complex domains, do not resolve particle scale physics that are important for combustion. Traditionally, bulk models are calibrated by adjusting the values of free parameters so that predictions match measured DDT data. These models typically incorporate empirical pressure dependent combustion sub-models, such as the "Forestfire" [88], Johnson-Tang-Forest [64], and "Ignition and Growth" sub-models [79]. Appropriately calibrated burn models can reproduce important initiation characteristics such as the variation in distance from the impactor surface where detonation first appears with respect to impact pressure. However, empirical burn rates are only accurate for a narrow range of applications in which the hot-spot distribution is similar to that for which it was calibrated; thus, bulk DDT models are not very predictive. Chemical kinetic rates are highly temperature dependent and will, therefore, be significantly affected by hot-spots formed at the sub-particle scale. Though recent developments in measurement techniques, such as laser-velocity interferometry [127], embedded magnetic gauges [120], and multifiber optical probes with streak photography [113], can resolve fast particle velocity fields, which have greatly enhanced our understanding of reactive wave behavior, meso-scale experimental measurements remain an outstanding scientific challenge.

In the absence of particle scale data, meso-scale computations have been recently performed to characterize the particle scale response $[5,7,10,16,17,33,60]$ and its effect on bulk behavior. These computations, generally performed over small domains, apply continuum equations to the heterogeneous meso-structure [67, 69, 71]. Studies vary in size from tens to hundreds of particles in 2D $[76,133,134]$, to sometimes thousands of multi-material particles in 3D [9]. The very large scale simulations often sacrifice numerical resolution in favor of physical complexity, as discussed

below. Several computational techniques, such as Eulerian hydrocodes [7, 24, 17], Lagrangian finite element codes [76, 137], and discrete particle codes [136, 32] have been used to characterize 
compaction of granular metals (e.g., aluminium, copper, alumina, etc.), sugar, and HMX. Mesoscale models have been applied to study both high pressure shock loading of particle ensembles $[7,60,95]$ and low pressure, weak compaction waves responsible for DDT [84, 95].

Traditionally, two classes of meso-scale models have been applied to the compaction of energetic solids. The first class utilizes discrete element or quasi-molecular dynamics techniques for which the meso-scale response is governed by classical equations of motion subject to heuristic, or even ad hoc, explicit force fields. Examples include the Smooth Particle Hydrodynamics (SPH) technique [81, 102]), the discrete element technique [33, 107, 136], and the Dissipative Particle Dynamics technique $[53,58]$. The SPH technique is mesh-free, and has proved useful for phenomenological modeling of detonation propagation, explosive fragmentation, and brittle fracture [117]. However, because SPH is an interpolation-based method, particle boundaries are not welldefined which presents difficulties in modeling surface phenomena such as friction. In discrete element techniques material mass is represented by a number of discrete elements whose motion is governed by classical dynamics. These methods assume that the thermomechanical response can be effectively represented by the aggregate motion of interconnected discrete elements and the evolution of their internal state parameters such as temperature and composition. However, these methods are incapable of describing spatial variations in thermomechanical fields within elements. Other mesh-free particle techniques developed within the finite element community include the Element Free Galerkin method [14] and the Reproducing Kernel Particle method [83]. The second class of models involves direct numerical integration of continuum field equations using finite-volume, finite-element, or finite-difference techniques that attempt to resolve thermal, mechanical, and chemical fields in stochastic geometries with multimaterial behavior. A fundamental approximation in this approach is the use of constitutive relations that are observed at the continuum level and extrapolated to the meso-scale. These techniques may be Lagrangian $[76,137]$, Eulerian [5, 9, 22, 93], or arbitrary Lagrangian-Eulerian (ALE) [30, 57], and they utilize computational grids over the spatial domain. Computational grids used with Lagrangian formulations deform with the material, whereas Eulerian methods use mostly stationary grids through 
which material flows. ALE methods encompass features of both the Lagrangian and Eulerian formulations. The numerical technique used in this study falls within this second class of models.

Most finite-element compaction models reported in the literature are based on Eulerian grid techniques. These techniques, which have been extensively used by Benson, et al [17, 22], to model complex, experimentally obtained grain structures, allow for relatively simple inclusion of large material deformations, phase change, irregular grain morphologies, and variable grain size distributions. Including such features within a Lagrangian framework would be complicated due to excessive grid distortion that can lead to numerical inaccuracies and instabilities. The Eulerian techniques have been applied to both simple [16] and sophisticated problems that require the use of hundreds of parallel processors to generate and analyze massive amounts of data [8]. Baer has extensively used the Eulerian shock physics code CTH (developed at Sandia National Laboratory) to construct and analyze complex, three-dimensional meso-structures having realistic packing arrangements and particles morphologies [5, 6, 7, 8, 9]. Menikoff and Kober [95] simulated the dynamic compaction of a small number of particles $(<500)$ using a 2D Eulerian code (COMADREJA) to characterize the structure of piston driven compaction waves; a similar code (RAVEN) has also been used to study the dynamic compaction of powdered metals [20, 21]. Other relevant studies include those of Borg and Vogler [24], and Lowe and Longbottom [85]. Despite certain advantages of Eulerian based techniques, their interfacial transport algorithms are computationally expensive (high CPU time) and they result in significant numerical diffusion near material/particle interfaces, particularly for problems involving small to moderate deformations. Unlike Lagrangian techniques, Eulerian techniques are incapable of accurately tracking particle boundaries in time, requiring the use of special algorithms to identify and reconstruct these boundaries which introduces numerical inaccuracies [20, 22]. Consequently, it is difficult to accurately describe surface phenomena, such as friction, within the context of these Euerlian based models.

Two-dimensional Lagrangian simulations of dynamic compaction of metal particles have been performed by Kumar, et al [76], for small-scale, symmetric ensembles using ABAQUS/Explicit. Their study revealed that frictional dissipation results in substantial localized temperature rises 
(>1000 K) near contact surfaces for piston speeds ranging from 300-1000 m/s, but their ensemble did not include a sufficient number of particles to examine compaction wave structure. Zavaliangos [137] also used ABAQUS/Explicit to analyze a larger ensemble containing 400 particles having a random packing arrangement, but focused only quasistatic deformation. Lewis, et al [80], used the finite element method to simulate a pharmaceutical powder tablet making process for irregularly shaped particles. They predicted that particle size, shape, and mechanical properties have a significant influence on quasistatic deformation. A separate class of meso-scale models recently developed are based on the Material Point Method (MPM) which represents a mixed EulerianLagrangian formulation that can accurately track particle interactions In this technique, particles carry micro-structural information that is interpolated onto a computational grid upon which the conservation equations are numerically integrated in time; the numerical solution is then reinterpolated back to the particles, the old grid is discarded in favor of a new, smooth grid, and the process is repeated. Contact constraints are enforced by eliminating differences between the interpolated particle and system velocity for each particle at each grid point. Bardenhagen, et al $[10,11]$, have used MPM based models to describe both the quasistatic and weak impact behavior of granular materials. This method eliminates the requirement of a separate contact detection algorithm, which are known to be extremely time consuming, and allows for excessive deformation of particles to be easily described without issues of mesh distortion. However, interpolation and reinterpolation of information between the material points and grid points introduces numerical errors which can accumulate in time. This method is still in the developmental stage, and it remains uncertain whether MPM can truly be applied to model extreme states of deformation that are encountered during dynamic compaction of granular solids.

A variety of physical phenomena have been incorporated into both Eulerian and Lagrangian meso-scale models to characterize potential hot-spot formation mechanisms within energetic solids and porous metals for a range of impact speeds. The effects of particle size distribution [85], morphology [9], and material properties [96, 98] on compaction wave behavior have been examined. However, the influence of inter-particle friction on compaction wave structure and hot-spot for- 
mation remains largely uncharacterized. Progress has been hampered by the inability of current meso-scale models (particularly Eulerian based models which are popular within the energetic solids community) to accurately resolve contact interfaces. Therefore, in this study, a combined finite-discrete element method is adapted and implemented to efficiently model the impact response of large viscoplastic and frictional particle ensembles. Individual particles are modeled as discrete elements, where each discrete element is discretized using finite elements to resolve thermomechanical fields within particles which influence their contact mechanics. Interactions between particles are modeled using a penalty based distributed potential force method, and particle deformation is described using a conventional finite element method. Munjiza and Andrew [103, 104, 105] used a similar method to model fragmentation resulting from the impact of a large number of frictionless rigid bodies. To the author's knowledge, this technique has not been previously applied to study frictional interactions between deformable particles. The accuracy of the combined finite-discrete element method hinges on the accuracy of the contact detection/interaction model. A good contact interaction model should result in realistic force fields on the particle boundaries while being energy consistent and computationally efficient.

Computational modeling of multi-body impact requires accurate enforcement of normal and/or frictional contact constraints on the boundaries of colliding bodies. Details on the development of thermodynamically consistent algorithms for frictionless and frictional contact problems can be found in the works of Laursen and co-workers [56, 77, 78, 94], Armero and Petrocz [2, 3], and Rieger and Wriggers [119]. Two methodologies are generally used to describe such phenomena. The first methodology, referred to as an impulse-momentum method, assumes that interactions between bodies occur sufficiently fast so that their packing configuration remains unaltered; as such, this method is ideally suited for rigid body impact. Coefficients of restitution and impulse ratios are often used with this method to account for energy transfer and dissipation [28, 29]. The second methodology assumes that interaction forces between bodies vary continuously during impact and that they depend on the local deformation state. Traditionally, penalty based $[39,67,69,70]$, Lagrange multiplier based [12, 72, 110, 122], and augmented Lagrange based [112] 
methods are routinely used to estimate these forces within the context of finite element techniques applied to the contacting bodies. These methods all require the minimization of a gap potential which is representative of inter-particle overlap. Penalty methods are advantageous in that they explicitly enforce contact constraints by penalizing the gap potential using a large value for a numerical penalty parameter. Penalty based methods are simple, computationally inexpensive, and robust; thus, they are ideal for use with large particle ensembles. However, these methods do not exactly enforce contact constraints, and choosing a suitable value for the penalty parameter is sometimes difficult and often application dependent. The Lagrange multiplier method exactly enforces contact constraints, but significantly increases computational costs associated with obtaining estimates for multiplier values. Augmented Lagrange methods combine features of both penalty and Lagrange multiplier methods, but are primarily used in implicit codes that are incapable of tracking wave behavior. Penalty based methods better describe complex system behavior, including the effects of inter-body friction $[39,74,87,109,130]$. Similar methods have been applied to impact problems by Bathe and Bouzinov [12], Farahani, et al [43], Heinstein, et al [56], and $\operatorname{Kim}[72]$.

\subsection{Objectives and Plan}

As mentioned, the primary objective of this study is to computationally characterize meso-scale temporal and spatial fluctuations in thermomechanical fields induced by mild impact of granular energetic solids because of their relevance to combustion initiation. Specific novel objectives of this study include:

1. To develop, numerically implement, and verify an efficient, explicit 2D Lagrangian finiteelement technique for the analysis of deformation wave propagation in heterogeneous solids. To this end, a conventional combined finite and discrete element technique is modified to more efficiently compute wave phenomena within large granular ensembles. Individual particles are resolved using triangular finite elements, finite deformation of particles is described by a suitable constitutive theory, and contact between particles is treated using an energy 
consistent, penalty based, distributed potential force method. Traditionally, this potential force method has been restricted to frictionless, rigid body impact problems; in this study, it is substantially modified to model frictional impact between deformable particles. This modification enables complex interactions between large particle ensembles do be described in a computationally efficient and realistic manner. Also, a new method is developed to enforce thermal contact constraints in a manner similar to that used to enforce kinematic constraints.

2. To computationally examine energy partitioning within a micro-particle cluster due to impact with a rigid, planar wall. Emphasis is placed on characterizing the temporal and spatial partitioning of energy within a close-packed cluster of well-resolved, micron-size particles in the vicinity of the wall, and their dependence on impact angle $\left(0^{\circ} \leq \phi^{\circ} \leq 80^{\circ}\right.$, where $\phi^{\circ}=0^{\circ}$ corresponds to normal impact). Sensitivity of the cluster response to particle size distribution and initial cluster configuration is demonstrated. This study is relevant to the ignition and combustion of shock-dispersed energetic clusters by impact with boundaries.

3. To computationally examine meso-scale energy partitioning within uniaxial, quasi-steady deformation waves due to constant speed piston impact. Particular emphasis is placed on characterizing the evolution of hot-spot mass fraction within the wave structure and its dependence on impact speed. The relative importance of plastic and friction work as hotspot mechanisms is identified and discussed. Quasi-1D bulk predictions are obtained and analyzed by averaging the meso-scale response over suitable material volumes.

4. To characterize the dependence of deformation wave structure and hot-spot formation on constitutive parameters and material meso-structure. Key constitutive parameters include the plastic viscosity, which establishes the time scale for rate-dependent relaxation of the stress state to the material yield surface, and the friction coefficient, which establishes the magnitude of the surface traction at inter-particle contact surfaces. Both of these parameters, which are difficult to experimentally determine under impact conditions, can significantly 
affect the particle and bulk-scale mechanics. Also, most energetic materials used in practice have a non-uniform particle size distribution so that high volumetric packing densities can be obtained. It may also be possible to promote a desired effect (e.g., impact sensitivity and combustion rate) by carefully controlling the initial particle size distribution of the material; this possibility requires a reasonable understanding of meso-scale physics. Therefore, simulations are also performed in this study for materials having different initial particle size distributions to demonstrate their effect on both deformation wave structure and hot-spot formation.

The plan of this dissertation is as follows. First, a mathematical description of multi-body impact is given in Chapter 2, including a discussion of finite strain kinematics, the governing conservation principles, and the constitutive theory used in this study. Next, details of the numerical technique used to computationally solve the multi-body impact problem are given in Chapter 3 . In this chapter, the discrete form of the governing equations are summarized to provide a framework for implementation of the finite element technique, and the numerical strategies used to estimate deformation and contact forces are described. The numerical technique is verified in Chapter 4 against analytical solutions for simple problems, and against numerical predictions obtained by the FEA software LS-Dyna for more complex problems. To this end, the discrete form of the governing equations is verified for elastic, elastic-viscoplastic, and elastic-viscoplastic-friction impact cases. Numerical convergence is also established in this chapter, and the sensitivity of model predictions to key numerical parameters is demonstrated. Readers who are primarily interested in predictions for the physical system can skip Chapters 2 and 4 without difficulty. Predictions for the impact of a micro-particle cluster with a rigid wall are given in Chapter 5, and predictions for uniaxial deformation waves are given in Chapters 6 and 7. Finally, conclusions and future work are given in Chapter 8. 


\section{Chapter 2}

\section{Mathematical Model}

Describing the impact response of 2D particulate solids requires that their time-dependent displacement and temperature fields be determined subject to their thermo-mechanical properties and contact constraints. To this end, a suitable constitutive theory is required to describe deformation behavior of the solids, as well as a sophisticated contact interaction model to track contact interfaces for normal and frictional traction forces. In this study, the dynamic compaction problem is modeled as a multi-particle contact problem, where, the contact problem for each particle $\Omega$ is posed as a coupled initial-boundary-value problem (IBVP) for the displacement field $\mathbf{u}: \bar{\Omega} \times \tau \rightarrow \mathrm{R}^{n}$, and the temperature field $T: \bar{\Omega} \times \tau \rightarrow \mathrm{R}$, which are described by the evolution of mass, momentum and energy equations within $\Omega$ over a time interval $\tau=[0, T]$. Here, $\bar{\Omega}$ represents the closure of $\Omega$. These evolution equations may be expressed mathematically in integral or differential form. In addition, they can be posed in the Lagrangian/material or Eulerian/current configuration. In the present study, the differential forms of the equations of motion are posed and solved for in the current configuration.

The following is an outline for this chapter. The governing conservation equations are presented first, followed by a brief overview of finite deformation kinematics, which is presented in Section 2.1. Next, details of the constitutive relations used to close the system of equations are presented in Section 2.2 and 2.3. Expressions describing the temporal evolution of the deformation energetics and the temperature field are presented in Section 2.4. Lastly, the relevant initial and boundary conditions are summarized in Section 2.5.

For an arbitrary particle $\Omega$ of mass $m$ at time $t$, the continuity equation is given by:

$$
\frac{d m}{d t}=\frac{d}{d t} \oint_{\Omega} \rho d v=0,
$$


where $\rho: \bar{\Omega} \times \tau \rightarrow \mathrm{R}$ is the current density and $v$ is the current volume occupied by $\Omega$. The conservation equation for the linear momentum in Lagrangian form is given by:

$$
\rho \frac{d}{d t} \mathbf{v}-\nabla_{x} \cdot \boldsymbol{\sigma}-\rho \mathbf{f}=0
$$

where $\boldsymbol{\nabla}_{x} \equiv \partial(\cdot) / \partial \mathbf{x}$ is the Lagrangian gradient operator in the current configuration, $\mathbf{v}: \bar{\Omega} \times \tau \rightarrow$ $\mathrm{R}^{n}$ is the local material velocity, $\mathbf{f}: \bar{\Omega} \times \tau \rightarrow \mathrm{R}^{n}$ is the body force and $\boldsymbol{\sigma}$ is the Cauchy stress tensor. Finally, the evolution equation for the internal energy in Lagrangian form is given by:

$$
\rho \frac{d u}{d t}=\boldsymbol{\sigma}: \mathbf{d}-\nabla_{x} \cdot \mathbf{q}+\rho r
$$

where $u: \bar{\Omega} \times \tau \rightarrow \mathrm{R}$ is the internal energy per unit mass, $r: \bar{\Omega} \times \tau \rightarrow \mathrm{R}$ is the heat generated per unit mass and $\mathbf{q}: \bar{\Omega} \times \tau \rightarrow \mathrm{R}^{n}$ is the heat flux. Here, $\mathbf{d}$ is the Eulerian deformation rate tensor defined as

$$
\mathbf{d} \equiv \frac{1}{2}\left(\boldsymbol{\nabla}_{x} \mathbf{v}+\left(\boldsymbol{\nabla}_{x} \mathbf{v}\right)^{T}\right)
$$

where the superscript $\mathrm{T}$ denotes the transpose operation. In addition to satisfying the equations of motion, each particle also has to satisfy the second law of thermodynamics. In the current configuration, the second law is expressed by the local Clausius-Duhem inequality as

$$
\rho \dot{\eta}-T^{-1}\left(\rho r-\nabla_{x} \cdot \mathbf{q}\right)+\mathbf{q} \cdot \nabla_{x} T^{-1} \geq 0
$$

where $\eta: \bar{\Omega} \times \tau \rightarrow \mathrm{R}$ is the entropy density.

\subsection{Finite Deformation Kinematics}

Using a small strain theory to describe motion of deformable particles assumes that the displacements and the displacement gradients are small compared to unity. However, for impact problems involving deformable bodies, such as the one considered in this study, substantially large strains $\left(10^{-2}-10^{0}\right)$ and deformation rates $\left(10^{5}-10^{7} s^{-1}\right)$ are commonly observed for impact 
speeds ranging from 50 to $500 \mathrm{~m} / \mathrm{s}$. As such, a linear description of the stress-strain behavior is inadequate to describe deformation and a finite deformation theory is needed to model material behavior.

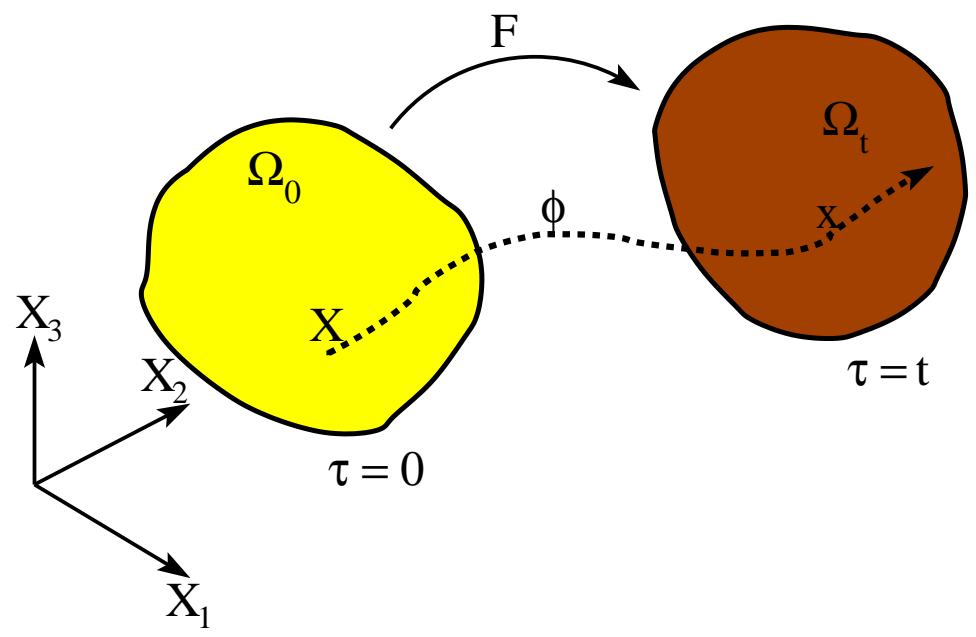

Figure 2.1: General motion of a deformable particle.

In the discussion that follows, key aspects of finite deformation theory are described briefly. A more complete description can be found in Reference [86]. In finite deformation theory, for any arbitrary particle $\Omega$ in motion, as illustrated in Fig. 2.1, relevant quantities such as density, stress and strain can be described in terms of where the particle was before deformation or where it is during deformation. The former is called the material/initial configuration $\Omega_{0}$, whereas the latter is called the spatial/current configuration $\Omega_{t}$. Its material points are denoted by $\mathbf{X}$, whereas its current position is located by the coordinates $\mathbf{x}$. An important quantity in this theory is the deformation gradient $\mathbf{F}$, which relates the relative position of two neighboring points before and after deformation. The deformation gradient is locally computed from the displacement vector field $\mathbf{u}$, and is given by $\mathbf{F}=\mathbf{1}+\boldsymbol{\nabla}_{0} \mathbf{u}$, where $\mathbf{1}$ is the second order unit tensor, $\boldsymbol{\nabla}_{0} \equiv \partial(\cdot) / \partial \mathbf{X}$ is the Lagrangian gradient operator with respect to the initial configuration, and $\mathbf{u}$ is computed as

$$
\mathbf{u}(\mathbf{X}, t)=\mathbf{x}-\mathbf{X}
$$

Also important, is the determinant of the deformation gradient, $J=\operatorname{det}[\mathbf{F}]$, which characterizes volumetric deformation; if the initial density of the particle is $\rho_{0}$, then the local density after 
deformation is given by $\rho=\rho_{0} / J$. In finite deformation theory, it is convenient and more natural to decompose $\mathbf{F}$ into purely rotational and stretch components as $\mathbf{F}=\mathbf{R U}=\mathbf{V R}$, where $\mathbf{U}$ and $\mathbf{V}$ are positive symmetric right and left stretch tensors, respectively, and $\mathbf{R}$ is the orthogonal rotation tensor. It is further convenient to express the stretch tensors in terms of their eigenvalues and eigenvectors as

$$
\mathbf{V}=\sum_{i=1}^{3} \lambda_{i} \mathbf{n}_{i}^{\prime} \otimes \mathbf{n}_{i}^{\prime}, \quad \mathbf{U}=\sum_{i=1}^{3} \lambda_{i} \mathbf{N}_{i}^{\prime} \otimes \mathbf{N}_{i}^{\prime}
$$

where $\left\{\lambda_{1}, \lambda_{2}, \lambda_{3}\right\}$ are their eigenvalues, and the symbol $\otimes$ represents the dyadic tensor product. Here, $\left\{\mathbf{N}_{1}^{\prime}, \mathbf{N}_{2}^{\prime}, \mathbf{N}_{3}^{\prime}\right\}$ and $\left\{\mathbf{n}_{1}^{\prime}, \mathbf{n}_{2}^{\prime}, \mathbf{n}_{3}^{\prime}\right\}$ are the orthogonal eigenvectors of $\mathbf{U}$ and $\mathbf{V}$, respectively, which are related to each other by $\mathbf{n}_{i}^{\prime}=\mathbf{R} \mathbf{N}_{i}^{\prime}$. This decomposition allows the deformation gradient to be described in terms of the eigenvalues and eigenvectors as

$$
\mathbf{F}=\sum_{i=1}^{3} \lambda_{i} \mathbf{n}_{i}^{\prime} \otimes \mathbf{N}_{i}^{\prime}
$$

\subsubsection{Strain}

In finite deformation kinematics strain can be described in the material and spatial configuration. A spatial strain measure is one in which the independent variables are given with respect to their current coordinates, whereas, a Lagrangian strain measure is one in which the independent variables are given with respect to their initial coordinates. Generalized material and spatial strain measures of order $m$ are defined as

$$
\mathbf{E}^{(m)}=\sum_{i=1}^{3} \frac{1}{m}\left(\lambda_{i}^{(m)}-1\right) \mathbf{N}_{i}^{\prime} \otimes \mathbf{N}_{i}^{\prime}, \quad \mathbf{e}^{(m)}=\sum_{i=1}^{3} \frac{1}{m}\left(1-\lambda_{i}^{(-m)}\right) \mathbf{n}_{i}^{\prime} \otimes \mathbf{n}_{i}^{\prime}
$$

respectively. In particular, the case $m \rightarrow 0$ gives the material and spatial logarithmic strain tensors as

$$
\mathbf{E}^{(0)}=\sum_{i=1}^{3} \ln \left(\lambda_{i}\right) \quad \mathbf{N}_{i}^{\prime} \otimes \mathbf{N}_{i}^{\prime}, \quad \mathbf{e}^{(0)}=\sum_{i=1}^{3} \ln \left(\lambda_{i}\right) \mathbf{n}_{i}^{\prime} \otimes \mathbf{n}_{i}^{\prime}
$$


respectively. In general, the material and spatial strain measures differ from each other. However, for small strains, both measures reduce to the conventional definition of the small strain tensor given by infinitesimal strain theory.

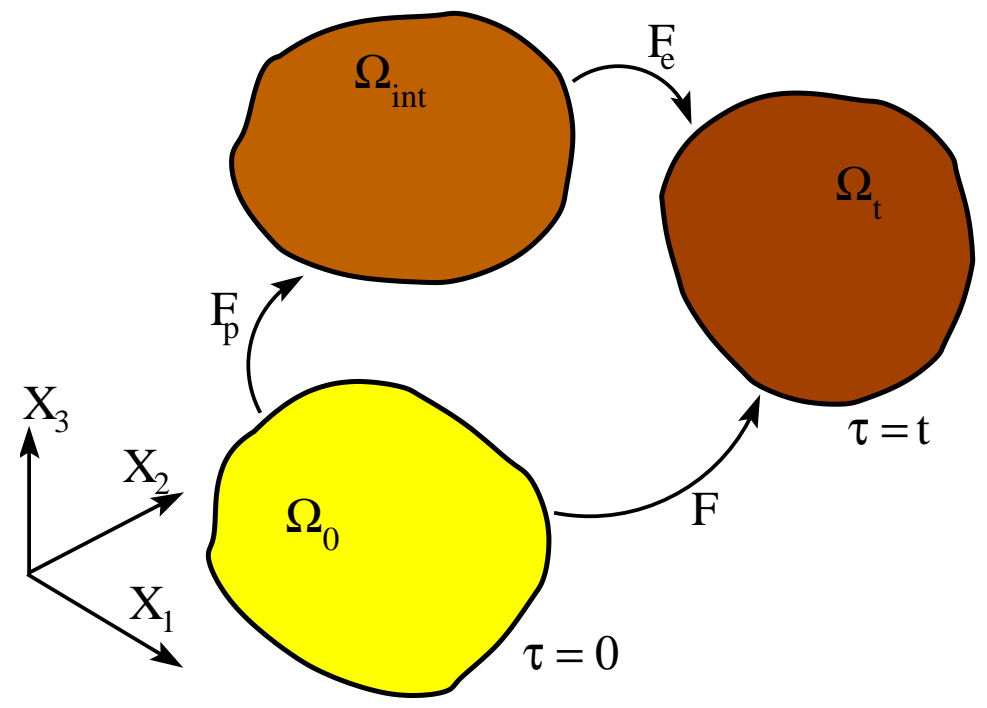

Figure 2.2: Multiplicative decomposition of the deformation gradient.

The general approach used to describe finite strain viscoplasticity is based on the multiplicative decomposition of deformation gradient tensor into its elastic and plastic components, $\mathbf{F}=$ $\mathbf{F}_{e} \mathbf{F}_{p}$, as illustrated by the mapping in Fig. 2.2. Here, the plastic component $\mathbf{F}_{p}$ results in a stress free intermediate configuration $\Omega_{\text {int }}$, and the elastic component $\mathbf{F}_{e}$ deforms this configuration into the current configuration $\Omega_{t}$ where equilibrium is imposed. Here, it should be noted that in general the deformation gradients $\mathbf{F}_{e}$ and $\mathbf{F}_{p}$ are not uniquely defined. This is because the intermediate configuration is not unique and can only be determined up to a rigid body rotation superimposed on the intermediate configuration preserving it unstressed. In this study, the intermediate configuration is fixed by requiring that the inelastic deformation have the same principal directions as the elastic deformation, that is, they have the same eigenvectors. This is a reasonable assumption for elastically isotropic materials. Additional details regarding this simplification can be found in References [23] and [94]. Based on this decomposition, the elastic component of the left Cauchy-Green tensor and the plastic component of the right Cauchy-Green tensor are defined 
by

$$
\mathbf{b}_{e}=\mathbf{F}_{e} \mathbf{F}_{e}^{T}=\sum_{i=1}^{3}\left(\lambda_{e, i}^{2}\right) \mathbf{n}_{i} \otimes \mathbf{n}_{i}, \quad \mathbf{C}_{p}=\mathbf{F}_{p}^{T} \mathbf{F}_{p}=\sum_{i=1}^{3}\left(\lambda_{p, i}^{2}\right) \mathbf{N}_{i} \otimes \mathbf{N}_{i}
$$

respectively, where $\lambda_{e, i}^{2}$ and $\lambda_{p, i}^{2}$ are the eigenvalues of $\mathbf{b}_{e}$ and $\mathbf{C}_{p}$, and $\mathbf{n}_{i}$ and $\mathbf{N}_{i}$ are their eigenvectors, respectively. In general, the eigenvectors $\mathbf{n}_{i}^{\prime}$ of $\mathbf{F}$ do not coincide with the eigenvectors of $\mathbf{b}_{e}$. However, because it is assumed that the inelastic and elastic deformation are collinear, the eigenvectors $\mathbf{n}_{i}^{\prime}$ and $\mathbf{N}_{i}^{\prime}$ coincide with $\mathbf{n}_{i}$ and $\mathbf{N}_{i}$, respectively. Subsequently, the eigenvalues of $\mathbf{b}_{e}$ and $\mathbf{C}_{p}$ can be related to the eigenvalues of $\mathbf{V}$ as $\lambda_{i}=\lambda_{e, i} \lambda_{p, i}$, which also facilitates the elastic and plastic components of $\mathbf{F}$ to be conveniently defined as

$$
\mathbf{F}_{e}=\sum_{i=1}^{3} \lambda_{e, i} \mathbf{n}_{i} \otimes \tilde{\mathbf{n}}_{i}, \quad \mathbf{F}_{p}=\sum_{i=1}^{3} \lambda_{p, i} \tilde{\mathbf{n}}_{i} \otimes \mathbf{N}_{i}
$$

respectively, where $\tilde{\mathbf{n}}_{i}$ are their eigenvectors in the intermediate configuration. Substituting this result in the second part of Eq. (2.10) gives the elastic and plastic component of the spatial logarithmic strain tensor as

$$
\mathbf{e}_{e}^{(0)}=\sum_{i=1}^{3} \ln \left(\lambda_{e, i}\right) \mathbf{n}_{i} \otimes \mathbf{n}_{i}, \quad \mathbf{e}_{p}^{(0)}=\mathbf{e}^{(0)}-\mathbf{e}_{e}^{(0)},
$$

respectively.

\subsubsection{Stress}

Stress can be first defined in the current configuration in the standard way as force per unit area, which is given by the Cauchy stress tensor $\boldsymbol{\sigma}$. A totally symmetric material stress tensor is given by the second Piola-Kirchhoff stress tensor $\mathbf{S}$, which represents the force per unit area in

the initial configuration, and is related to the Cauchy stress tensor as $\mathbf{S}=J \mathbf{F}^{-1} \boldsymbol{\sigma} \mathbf{F}^{-T}$. It is convenient to define another stress tensor in the current configuration which is the Kirchhoff stress tensor $\boldsymbol{\tau}=J \boldsymbol{\sigma}$. The Kirchhoff stress tensor can also be obtained from the second Piola-Kirchhoff stress tensor in the following manner

$$
\boldsymbol{\tau}=\phi_{*}[\mathbf{S}]
$$


where for any material object, such as $\mathbf{S}$, the operation $\phi_{*}[\mathbf{S}]=\mathbf{F}^{-T} \mathbf{S} \mathbf{F}^{-1}$ represents a push forward of $\mathbf{S}$ from the initial configuration to the current configuration. Similarly, for any spatial object, such as $\boldsymbol{\tau}$, the inverse operation $\phi_{*}^{-1}[\boldsymbol{\tau}]=\mathbf{F}^{T} \boldsymbol{\tau} \mathbf{F}$ represents the pull back of a spatial object $\boldsymbol{\tau}$ from the current configuration to the initial configuration. As seen later, push forward and pull back operations are frequently used in finite deformation kinematics to move between the current and initial configuration.

\subsection{Hyperelastic Formulation of Elastic-Viscoplasticity}

The equations of motion for any particle depend on the stress state within the particle. Because these stresses result from the deformation of the material, it is necessary to express them in terms of strain. To this end, a hyperelastic formulation is adopted, whereby stresses are derived from the Helmholtz free energy density $\Psi$ using strain. In the remainder of this section, key aspects of this formulation are discussed briefly and the expression for $\sigma$ is derived in terms of $\Psi$. A more complete description can be found in Reference [86]. In passing, it is noted that in literature another potential called the stored energy potential $\tilde{\Psi}$ is also commonly used to derive the stress response function. This potential is related to the Helmholtz free energy density as $\tilde{\Psi}=\rho_{0} \Psi$, and it facilitates the constitutive theory to be described in terms of the Kirchhoff stress instead of the Cauchy stress. In finite strain thermoelastic-viscoplasticity, $\Psi$ is defined as a function of state variables $\mathbf{b}_{e}$, the effective plastic strain $\epsilon_{p}$ and $T$. $\Psi$ can be expressed in terms of $u$ and $\eta$ using the Maxwell relation:

$$
\Psi\left(\mathbf{b}_{e}, \epsilon_{p}, T\right) \equiv u-T \eta
$$

where the entropy density $\eta$ can be derived from $\Psi$ as $\eta=-\partial \Psi / \partial T$. In the current configuration, the second law is expressed by the local Clausius-Duhem inequality given by Eq. (2.5). Using Eq. (2.3), the term in the parenthesis in Eq. (2.5) may be replaced to give

$$
\rho \dot{\eta}-T^{-1}(\rho \dot{u}-\boldsymbol{\sigma}: \mathbf{d})+\mathbf{q} \cdot \nabla_{x} T^{-1} \geq 0 .
$$


Assuming that the heat flux is given by a generalized Fourier law and recognizing that heat always flows from regions of higher temperature to lower temperature, that is $\mathbf{q} \cdot \boldsymbol{\nabla}_{x} T^{-1} \geq 0$, the more restrictive form of the second law is given as

$$
\mathcal{D} \equiv \rho T \dot{\eta}+\boldsymbol{\sigma}: \mathbf{d}-\rho \dot{u} \geq 0
$$

where $\mathcal{D}$ is the total dissipation.

Using Eq. (2.15), the time derivative of $\Psi$ can be expressed as

$$
\dot{\Psi}=\dot{u}-T \dot{\eta}-\eta \dot{T}
$$

Alternatively, using the chain rule, the time derivative of $\Psi$ is also given by

$$
\dot{\Psi}=\frac{\partial \Psi}{\partial \mathbf{b}_{e}} \dot{\mathbf{b}}_{e}+\frac{\partial \Psi}{\partial T} \dot{T}+\frac{\partial \Psi}{\partial \epsilon_{p}} \dot{\epsilon_{p}}
$$

Here, the objective material time derivative of $\mathbf{b}_{e}$ is given by

$$
\dot{\mathbf{b}}_{e}=2 \mathbf{d b}_{e}+L_{\phi}\left(\mathbf{b}_{e}\right)
$$

where $L_{\phi}\left(\mathbf{b}_{e}\right)$ is the Lie derivative of $\mathbf{b}_{e}$ defined as

$$
L_{\phi}\left[\mathbf{b}_{e}\right]=\phi_{*}\left[\frac{d}{d t}\left(\phi_{*}^{-1}\left[\mathbf{b}_{e}\right]\right)\right]
$$

Substituting for $\dot{u}$ in Eq. (2.17) using Eq. (2.18), and then for $\dot{\Psi}$ using Eq. (2.19), and after some rearrangement, the expression for the total dissipation $\mathcal{D}$ is given as

$$
\mathcal{D} \equiv\left(\boldsymbol{\sigma}-2 \rho \frac{\partial \Psi}{\partial \mathbf{b}_{e}} \mathbf{b}_{e}\right): \mathbf{d}+2 \rho \frac{\partial \Psi}{\partial \mathbf{b}_{e}} \mathbf{b}_{e}: \mathbf{d}_{p}-\rho \frac{\partial \Psi}{\partial \epsilon_{p}} \dot{\epsilon_{p}} \geq 0
$$


where $\mathbf{d}_{p}$ is the Eulerian inelastic rate of deformation defined as

$$
\mathbf{d}_{p} \equiv \frac{-1}{2} L_{\phi}\left(\mathbf{b}_{e}\right) \mathbf{b}_{e}{ }^{-1}
$$

For a purely elastic process, the internal plastic variables do not change with time, that is, $\mathbf{d}_{p}=\dot{\epsilon_{p}}=0$. Using this result in Eq. (2.22) gives the constitutive relation for the Cauchy stress tensor as

$$
\boldsymbol{\sigma}=2 \rho \frac{\partial \Psi}{\partial \mathbf{b}_{e}} \mathbf{b}_{e}
$$

Also, assuming that the same constitutive equation remains valid for the plastic process and substituting the previous result in Eq. (2.22) gives the final form of the second law, which must be satisfied by the constitutive theory used in this study, as

$$
\mathcal{D} \equiv \boldsymbol{\sigma}: \mathbf{d}_{p}-\rho \frac{\partial \Psi}{\partial \epsilon_{p}} \dot{\epsilon_{p}} \geq 0
$$

\subsubsection{Isotropic Material Behavior}

In this study, an isotropic formulation of the hyperelastic thermoelastic-viscoplasticity theory is used to describe the constitutive behavior. Implications of this assumption on the constitutive theory are now discussed. Isotropy entails that the relationship between the free energy $\Psi$ and $\mathbf{b}_{e}$ be independent of the material axes chosen. Consequently, $\Psi$ must only be a function of the invariants of $\mathbf{b}_{e}$ and therefore of the form $\Psi\left(I_{\mathbf{b}_{e}}, I I_{\mathbf{b}_{e}}, I I I_{\mathbf{b}_{e}}, \boldsymbol{\kappa}, T\right)$, where $I_{\mathbf{b}_{e}}, I I_{\mathbf{b}_{e}}, I I I_{\mathbf{b}_{e}}$ are the invariants of $\mathbf{b}_{e}$ given by

$$
I_{\mathbf{b}_{e}}=\lambda_{e, 1}^{2}+\lambda_{e, 2}^{2}+\lambda_{e, 3}^{2}, \quad I I_{\mathbf{b}_{e}}=\lambda_{e, 1}^{4}+\lambda_{e, 2}^{4}+\lambda_{e, 3}^{4}, \quad I I I_{\mathbf{b}_{e}}=\left(\lambda_{e, 1} \lambda_{e, 2} \lambda_{e, 3}\right)^{2}
$$


Also, for an isotropic material, the eigenvectors of the Cauchy stress tensor are collinear with the eigenvectors of $\mathbf{b}_{e}$. Subsequently, $\boldsymbol{\sigma}$ can also be decomposed as

$$
\boldsymbol{\sigma}=\sum_{i=1}^{3}\left(\sigma_{i i}\right) \mathbf{n}_{i} \otimes \mathbf{n}_{i}
$$

where $\sigma_{i i}$ are the eigenvalues of $\boldsymbol{\sigma}$ defined as

$$
\sigma_{i i}=\rho \frac{\partial \Psi}{\partial \ln \left(\lambda_{e, i}\right)} .
$$

The analogous expression for the Kirchhoff stress can be obtained from the above analysis by multiplying Eq. (2.24) with $J$ to give

$$
\boldsymbol{\tau}=\sum_{i=1}^{3}\left(\tau_{i i}\right) \mathbf{n}_{i} \otimes \mathbf{n}_{i}
$$

where $\tau_{i i}$ are the eigenvalues of $\boldsymbol{\tau}$ defined as

$$
\tau_{i i}=\frac{\partial \tilde{\Psi}}{\partial \ln \left(\lambda_{e, i}\right)}
$$

\subsection{Constitutive Model}

Constitutive relations describing the stress response to deformation are presented in this section. For convenience, the following discussion is cast in terms of the stored energy potential and the Kirchhoff stress tensor rather than the free energy and Cauchy stress tensor. In this study, an isotropic finite strain theory is coupled with a viscoplastic overstress model to estimate the stress field within particles. A Von Mises type yield condition and an associative flow law is used to describe the evolution of plastic variables. Von Mises plasticity with linear isotropic hardening is defined by a temperature independent yield surface $\phi$, which is a function of $\boldsymbol{\tau}$ and a hardening 
parameter $q$ given by

$$
\phi(\boldsymbol{\tau}, q) \equiv \sqrt{\overline{\boldsymbol{\tau}}: \overline{\boldsymbol{\tau}}}-\sqrt{\frac{2}{3}}\left(\tau_{0}-q\right) \leq 0, \quad q=-\frac{\partial \tilde{\Psi}}{\partial \epsilon_{p}}
$$

where $\tau_{0}$ is the initial yield strength of the material and $\overline{\boldsymbol{\tau}}$ is the deviatoric component of the Kirchhoff stress. The evolution of plastic variables is described using an associative flow law. To this end, the principle of maximum plastic dissipation is invoked, which states that among all the admissible values of variables $(\boldsymbol{\tau}, q)$, that is, which satisfy the yield criterion, the optimal values are those which maximize the total dissipation given by Eq. (2.25). Mathematically, this is formulated as a constrained minimization problem by means of the Lagrange multiplier procedure as

$$
\mathcal{L}^{p}(\boldsymbol{\tau}, q)=\min [\boldsymbol{\tau}, q] \max [\dot{\epsilon}]\{-\mathcal{D}(\boldsymbol{\tau}, q)+\dot{\epsilon} \phi(\boldsymbol{\tau}, q)\}
$$

where $\dot{\epsilon}$ is the Lagrange or consistency parameter. Applying the associated Kuhn-Tucker optimality conditions result in

$$
\frac{\partial \mathcal{L}^{p}}{\partial \boldsymbol{\tau}}=-\mathbf{d}_{p}+\dot{\epsilon} \frac{\partial \phi(\boldsymbol{\tau}, q)}{\partial \boldsymbol{\tau}}=0, \quad \frac{\partial \mathcal{L}^{p}}{\partial q}=-\dot{\epsilon_{p}}+\dot{\epsilon} \frac{\partial \phi(\boldsymbol{\tau}, q)}{\partial q}=0 .
$$

Simplifying the above result gives the plastic evolution equations as

$$
\mathbf{d}_{p}=\dot{\epsilon} \frac{\partial \phi(\boldsymbol{\tau}, q)}{\partial \boldsymbol{\tau}}=\dot{\epsilon} \frac{\overline{\boldsymbol{\tau}}}{\sqrt{\overline{\boldsymbol{\tau}}: \overline{\boldsymbol{\tau}}}}, \quad \dot{\epsilon_{p}}=\dot{\epsilon} \frac{\partial \phi(\boldsymbol{\tau}, q)}{\partial q}=\sqrt{\frac{2}{3}} \dot{\epsilon}
$$

In passing, it is noted that by construction the trace of $\mathbf{d}_{p}=0$, which implies that all volume changes are entirely elastic, that is, $\operatorname{det}\left[\mathbf{F}_{p}\right]=1$. Subsequently, $\operatorname{det}\left[\mathbf{F}_{e}\right]=\operatorname{det}[\mathbf{F}]=J$. As such, the elastic stretches can be decomposed into volumetric and isochoric components as $J^{1 / 3}$ and $\bar{\lambda}_{e, i}=J^{-1 / 3} \lambda_{e, i}$, respectively, which facilitates the decomposition of $\boldsymbol{\tau}$ into purely volumetric and deviatoric components as

$$
\boldsymbol{\tau}=J p \mathbf{1}+\overline{\boldsymbol{\tau}}, \quad \overline{\boldsymbol{\tau}}=\sum_{i=1}^{3}\left(\bar{\tau}_{i i}\right) \mathbf{n}_{i} \otimes \mathbf{n}_{i}
$$


where the volumetric component $p$ (pressure) and the principal deviatoric component $\bar{\tau}_{i i}$ are given by

$$
p=\frac{1}{J} \frac{\partial \tilde{\Psi}}{\partial \ln (J)}, \quad \bar{\tau}_{i i}=\frac{\partial \tilde{\Psi}}{\partial \ln \left(\bar{\lambda}_{e, i}\right)},
$$

respectively.

For impact events, the inelastic material response of HMX is strain rate dependent. Therefore, a Perzyna type viscoplastic flow model is used to model the deformation behavior. Contrary to the rate independent model, where the admissible stress states are constrained to remain on or within the elastic domain, the rate dependent model is characterized by the effective stress, defined as $\tau_{e}=\sqrt{\frac{3}{2} \overline{\boldsymbol{\tau}}: \overline{\boldsymbol{\tau}}}$, no longer being constrained to be less than or equal to the yield stress. In this case, the overstress is defined as $d \equiv \tau_{e}-\left(\tau_{0}-q\right)$, where the brackets $\langle x\rangle$ are called the Macaulay brackets, defined as $\langle x\rangle=0.5(x+|x|)$. For Perzyna type viscoplastic models, the consistency parameter is given by

$$
\dot{\epsilon}=\sqrt{\frac{3}{2}}\left\langle\frac{\tau_{e}-\left(\tau_{0}-q\right)}{\gamma}\right\rangle,
$$

where $\gamma$ is the plastic viscosity. The above equation can be simplified and rearranged such that in the viscoplastic range, a new constraint can be defined, which is the generalization of the classical Von Mises criterion for rate dependent models, as

$$
\bar{\phi}\left(\boldsymbol{\tau}, \epsilon_{p}\right)=\tau_{e}-\left(\tau_{0}-q\right)-\sqrt{\frac{2}{3}}(\gamma \dot{\epsilon})=0
$$

In this study, a simple stretch based stored energy potential is assumed to model the material stress response. This is of the form

$$
\tilde{\Psi}\left(\boldsymbol{\lambda}_{e}, \epsilon_{p}, T\right)=\tilde{\Psi}^{e d}\left(\overline{\boldsymbol{\lambda}}_{e}\right)+\tilde{\Psi}^{e v}(J)+\tilde{\Psi}^{e t}(J, T)+\tilde{\Psi}^{i}\left(\epsilon_{p}\right)+\tilde{\Psi}^{T}(T)
$$

where $\tilde{\Psi}^{e d}$ is the isochoric component of $\tilde{\Psi}$ given by

$$
\tilde{\Psi}^{e d}\left(\overline{\boldsymbol{\lambda}}_{e}\right)=G \sum_{i=1}^{3}\left(\ln \left(\bar{\lambda}_{e, i}\right)\right)^{2}
$$


where $G$ is the shear modulus. $\tilde{\Psi}^{e v}$ is the volumetric component given by

$$
\tilde{\Psi}^{e v}(J)=\frac{k}{2}(\ln (J))^{2}
$$

where $k$ is the bulk modulus. $\tilde{\Psi}^{e t}(J, T)$ is the thermoelastic component given by

$$
\tilde{\Psi}^{e t}(J, T)=\left(T-T_{0}\right)\left(-3 \alpha\left(\frac{\partial \tilde{\Psi}^{e v}}{\partial J}\right)\right)
$$

where $\alpha$ is the linear coefficient of thermal dilatation and $T_{0}$ is a reference temperature. $\tilde{\Psi}^{i}\left(\epsilon_{p}\right)$ is the inelastic hardening component given by

$$
\tilde{\Psi}^{i}\left(\epsilon_{p}\right)=\frac{1}{2} h \epsilon_{p}^{2}
$$

where $h$ is the hardening modulus. Finally, $\tilde{\Psi}^{T}(T)$ is the purely temperature dependent component of the stored energy potential given by

$$
\tilde{\Psi}^{T}(T)=\rho_{0} c_{v}\left(\left(T-T_{0}\right)-T \ln \left(\frac{T}{T_{0}}\right)\right)
$$

where $c_{v}$ is the specific heat at constant volume. Finally, using Eq. (2.36) and the stored energy potential given by Eq. (2.39), the principal volumetric and deviatoric components of $\boldsymbol{\tau}$ are given by

$$
p=\frac{1}{J}\left(k \ln (J)-3 k \alpha\left(T-T_{0}\right) \frac{(1-\ln (J))}{J}\right), \quad \bar{\tau}_{i i}=2 G \ln \left(\bar{\lambda}_{e, i}\right),
$$

respectively.

\subsection{Deformation Energetics}

A key objective of this study is to characterize the partitioning of energy within the quasisteady compaction wave structure and to examine the relative importance of plastic work and friction work as hot-spot mechanisms. Therefore, an overview of the energetics described in 
this study is presented in this section. The total energy of any arbitrary particle undergoing deformation can be first partitioned into kinetic energy and internal energy. The internal energy, given by Eq. (2.3), can change due to heat and work contributions. In this study, the work contribution is solely due to impact induced deformation, which is the deformation power, given by

$$
\mathcal{P}=\frac{1}{\rho_{0}}(\boldsymbol{\tau}: \mathbf{d})
$$

Partitioning $\mathbf{d}$ into elastic and plastic components as $\mathbf{d}=\mathbf{d}_{e}+\mathbf{d}_{p}$, respectively, where $\mathbf{d} \equiv$ $0.5\left(\dot{\mathbf{F F}}^{-1}+\left(\dot{\mathbf{F F}}{ }^{-1}\right)^{T}\right)$ and $\mathbf{d}_{p}$ is given by Eq. (2.23), and partitioning $\boldsymbol{\tau}$ into its volumetric and deviatoric components using Eq. (2.35) the deformation power may be expressed as

$$
\mathcal{P}=\frac{1}{\rho_{0}}\left(\overline{\boldsymbol{\tau}}:\left(\mathbf{d}-\mathbf{d}_{p}\right)\right)+\frac{1}{\rho_{0}}\left(\boldsymbol{\tau}: \mathbf{d}_{p}\right)+\frac{J p}{\rho_{0}}\left(\mathbf{1}:\left(\mathbf{d}-\mathbf{d}_{p}\right)\right) .
$$

The first term on the right side of this equation accounts for non-thermal increases in shear strain energy due to shear work, whereas the remaining terms account for deformation induced heating due to plastic and compression work.

Using the above results, for an arbitrary particle $\Omega$, the temporal evolution of the total deformation work is obtained by first integrating Eq. (2.46) with respect to time and then over its mass $m$ (per unit depth) to give

$$
W_{d}(t)=\int_{m} \int_{0}^{t} \frac{1}{\rho_{0}}(\boldsymbol{\tau}: \mathbf{d}) d t d m
$$

Expressions for the instantaneous total elastic shear strain energy, compression work and plastic work are given by

$$
W_{s}(t)=\int_{m} \int_{0}^{t} \frac{1}{\rho_{0}}\left(\overline{\boldsymbol{\tau}}:\left(\mathbf{d}-\mathbf{d}_{p}\right)\right) d t d m, \quad W_{c}(t)=\int_{m} \int_{0}^{t} \frac{J p}{\rho_{0}}\left(\mathbf{1}:\left(\mathbf{d}-\mathbf{d}_{p}\right)\right) d t d m
$$

and

$$
W_{p}(t)=\int_{m} \int_{0}^{t} \frac{1}{\rho_{0}}\left(\boldsymbol{\tau}: \mathbf{d}_{p}\right) d t d m
$$


respectively. Here, the total elastic work is simply given by the sum of the shear strain energy and compression work as

$$
W_{e}(t)=\int_{m} \int_{0}^{t} \frac{1}{\rho_{0}}\left(\boldsymbol{\tau}:\left(\mathbf{d}-\mathbf{d}_{p}\right)\right) d t d m
$$

Expression for the instantaneous kinetic energy is given by

$$
E_{k}(t)=\int_{m} \frac{\mathbf{v}(t) \cdot \mathbf{v}(t)}{2} d m
$$

Although not discussed here, another important form of work which influences thermal energy is the friction work. In this study, friction is interpreted as a contact boundary condition and will be discussed in Section 2.5. The instantaneous total friction work is given by

$$
W_{f}(t)=\int_{\cup \Gamma_{c}} \int_{0}^{t} \mathbf{t}_{c} \cdot \mathbf{v}_{\mathbf{r}} d t d s
$$

where $\mathbf{t}_{c}$ is the traction force arising due to contact, $\mathbf{v}_{\mathbf{r}}$ is the corresponding relative velocity between surfaces in contact and $\cup \Gamma_{c}$ is the union of all contact surfaces.

The evolution equation for temperature can be derived from the local energy balance given by Eq. (2.3) and basic thermodynamic principles. For brevity, the details of this analysis are excluded and only the final result is presented. Assuming that the mechanical and thermal material properties are independent of temperature, the evolution equation for temperature is given by

$$
\rho c_{v} \frac{d T}{d t}=-\nabla_{x} \cdot \mathbf{q}+\rho r+\dot{Q}^{e}+\dot{Q}^{i}
$$

where $\dot{Q}^{e}$ and $\dot{Q}^{i}$ represent thermoelastic and inelastic contributions to heating given by

$$
\dot{Q}^{e}=-\frac{3 k \alpha T(1-\ln (J))}{J^{2}}\left(\mathbf{1}:\left(\mathbf{d}-\mathbf{d}_{p}\right)\right)
$$


and

$$
\dot{Q}^{i}=\boldsymbol{\sigma}: \mathbf{d}_{p}-\rho\left(h \epsilon_{p} \dot{\epsilon}_{p}\right)
$$

respectively. Here, q is estimated from Fourier's law as

$$
\mathbf{q}=-k_{T} \boldsymbol{\nabla}_{x} T
$$

where $k_{T}$ is the thermal conductivity of the material.

\subsection{Initial and Boundary Conditions}

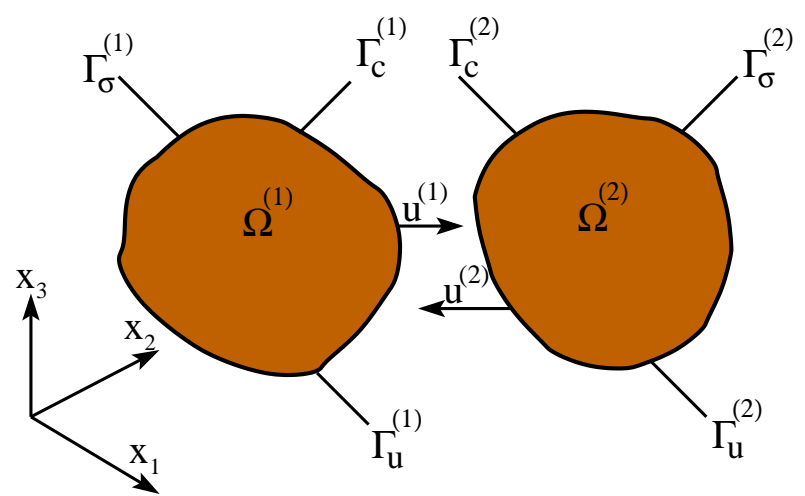

(a)

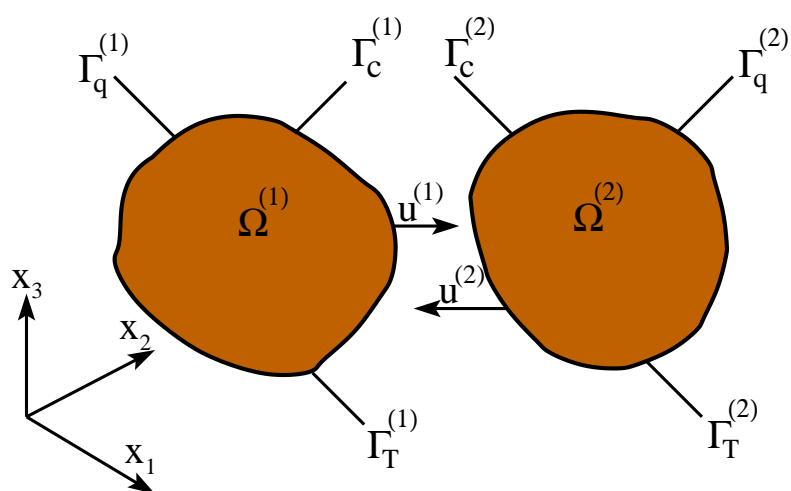

(b)

Figure 2.3: Boundary conditions of the two particle contact problem on the (a) Displacement field (b) Temperature field.

Initial and boundary conditions required to solve the IBVP are presented in this section. The following discussion is split into two segments. Initial and boundary conditions on the displacement field are presented first followed by the temperature field.

Fig. 2.3 illustrates contact between two deformable particles $\Omega^{(i)}(\mathrm{i}=1,2)$ over a time interval $\tau=[0, \mathrm{~T}]$; although the mathematical description given here is restricted to two-particle contact for convenience, it can be extended to multi-particle contact in a straightforward manner. Points within $\bar{\Omega}^{(1)}$ are denoted by $\mathbf{x}$, and points within $\bar{\Omega}^{(2)}$ by $\mathbf{y}$. The boundary of each particle $\Gamma^{(i)}$ is divided into three regions, $\Gamma_{\sigma}^{(i)}$ where tractions are prescribed, $\Gamma_{u}^{(i)}$ where displacements are prescribed, and $\Gamma_{c}^{(i)}$ is the contact boundary, such that, $\Gamma_{\sigma}^{(i)} \cup \Gamma_{u}^{(i)} \cup \Gamma_{c}^{(i)}=\Gamma^{(i)}$ and $\Gamma_{\sigma}^{(i)} \cap \Gamma_{u}^{(i)}=$ 
$\Gamma_{u}^{(i)} \cap \Gamma_{c}^{(i)}=\Gamma_{c}^{(i)} \cap \Gamma_{\sigma}^{(i)}=\emptyset$, where $\emptyset$ is the empty set. Initial and boundary conditions for the displacement field are given by

$$
\begin{array}{r}
\boldsymbol{\sigma}^{(i)} \cdot \mathbf{n}_{\sigma}^{(i)}=\overline{\mathbf{t}}_{\sigma}^{(i)} \text { on } \Gamma_{\sigma}^{(i)} \forall t \in \tau, \\
\mathbf{u}^{(i)}=\overline{\mathbf{u}}^{(i)} \text { on } \Gamma_{u}^{(i)} \forall t \in \tau, \\
\dot{\mathbf{u}}^{(i)}=\mathbf{v}_{\mathbf{0}}^{(i)} \text { on } \Omega^{(i)} \text { at } t=0, \\
\mathbf{u}^{(i)}=\mathbf{u}_{\mathbf{0}}{ }^{(i)} \text { on } \Omega^{(i)} \text { at } t=0,
\end{array}
$$

where $\overline{\mathbf{t}}_{\sigma}^{(i)}: \Gamma_{\sigma}^{(i)} \times \tau \rightarrow \mathrm{R}^{n}$ and $\overline{\mathbf{u}}^{(i)}: \Gamma_{u}^{(i)} \times \tau \rightarrow \mathrm{R}^{n}$ are prescribed traction and displacement vectors, respectively, $\mathbf{n}_{\sigma}^{(i)}$ is the unit outward normal vector to $\Gamma_{\sigma}^{(i)}$, and $\mathbf{v}_{\mathbf{0}}^{(i)}: \bar{\Omega}^{(i)} \rightarrow \mathrm{R}^{n}$ and $\mathbf{u}_{\mathbf{0}}{ }^{(i)}: \bar{\Omega}^{(i)} \rightarrow \mathrm{R}^{n}$ are the initial velocity and displacement fields. In this analysis, no initial displacement is assumed so that $\mathbf{u}_{\mathbf{0}}{ }^{(i)}=\mathbf{0}$. Also, no traction or displacement boundary conditions are prescribed so that $\Gamma_{\sigma}^{(i)}=\Gamma_{u}^{(i)}=\emptyset$. Therefore, the IBVP for each particle is purely driven by contact forces.

To describe the boundary conditions for the temperature field the boundary of $\Gamma^{(i)}$ is divided into three regions: $\Gamma_{T}^{(i)}$ where temperature is prescribed, $\Gamma_{q}^{(i)}$ where heat flux is prescribed, and $\Gamma_{c}^{(i)}$ is the contact boundary, such that, $\Gamma_{T}^{(i)} \cup \Gamma_{q}^{(i)} \cup \Gamma_{c}^{(i)}=\Gamma^{(i)}$ and $\Gamma_{T}^{(i)} \cap \Gamma_{q}^{(i)}=\Gamma_{q}^{(i)} \cap \Gamma_{c}^{(i)}=\Gamma_{c}^{(i)} \cap \Gamma_{T}^{(i)}=$ $\emptyset$. The initial and boundary conditions for the temperature field are given by

$$
\begin{array}{r}
T^{(i)}=\bar{T}^{(i)} \text { on } \Gamma_{T}^{(i)} \forall t \in \tau, \\
\left(-k_{T} \boldsymbol{\nabla}_{\boldsymbol{x}} T^{(i)}\right) \cdot \mathbf{n}_{q}^{(i)}=\bar{q} \text { on } \Gamma_{q}^{(i)} \forall t \in \tau, \\
T^{(i)}=T_{0} \text { on } \bar{\Omega}^{(i)} \text { at } t=0,
\end{array}
$$

where $\mathbf{n}_{q}^{(i)}$ is the unit outward normal to $\Gamma_{q}^{(i)}$ and $T_{0}$ is the initial reference temperature. In this study, no temperature boundary conditions are prescribed so that $\Gamma_{T}^{(i)}=\emptyset$. Also, adiabatic condition is imposed on $\Gamma_{q}^{(i)}$ so that $\bar{q}=0$. 


\subsubsection{Contact Boundary Conditions}

Traditionally, contact/impact problems are modeled as a constrained minimization problem, wherein, the potential energy of the colliding particles is minimized subject to contact constraints. There are various means to implement this numerically, which are discussed more explicitly in the next chapter. In the discussion that follows, only the mathematical interpretation of this approach is presented.

Contact constraints on $\Gamma_{c}^{(i)}$ are imposed on both the displacement and temperature fields. To impose kinematic constraints on $\Gamma_{c}^{(i)}$, a gap function is defined that locally quantifies the extent of inter-particle penetration at $\mathbf{x} \in \Gamma_{c}^{(1)}$ :

$$
g(\mathbf{x})=(\mathbf{x}-\overline{\mathbf{y}}(\mathbf{x})) \cdot \mathbf{n}_{c}^{(1)}(\mathbf{x})
$$

where $\mathbf{n}_{c}^{(1)}$ is the outward normal to $\Gamma_{c}^{(1)}$ and $\overline{\mathbf{y}}(\mathbf{x})$ is the closest point projection of $\mathbf{x}$ onto $\Gamma_{c}^{(2)}$. In a Euclidean sense, the closest point projection of points $\mathbf{x}$ to $\Gamma_{c}^{(2)}$ is defined by $\overline{\mathbf{y}}(\mathbf{x})=\arg \min (\|$ $\mathbf{x}-\mathbf{y} \|) \forall \mathbf{y} \in \Gamma_{c}^{(2)}$. Defining the local compressive normal traction on the contact surface by $t_{N}^{(1)}(\mathbf{x})=-\left(\boldsymbol{\sigma}^{(1)}(\mathbf{x}) \cdot \mathbf{n}_{c}^{(1)}(\mathbf{x})\right) \cdot \mathbf{n}_{c}^{(1)}(\mathbf{x})$, which is positive in compression, then contact constraints are given by the Kuhn-Tucker optimality conditions: $g \geq 0, t_{N} \geq 0$, and $t_{N} g=0$. The first of these constraints requires that the particles be impenetrable, the second requires that contact interaction be compressive and the third (complementarity condition) requires that compressive normal tractions only be induced by contact. Also, during persistent contact $t_{N} \dot{g}=0$, where $\dot{g}$ is the time derivative of the gap function. This condition is called the persistency condition which imposes an additional constraint on the velocity field.

When friction occurs, the influence of stick-slip behavior at the contact surface on its traction must be considered. To this end, the frictional traction vector $\mathbf{t}_{t}^{(1)}$ is estimated by AmontonsCoulomb law of dry friction for which particle stick or slip is possible depending on the system dynamics. A contact is assumed to stick if $\phi_{t} \equiv\left|\mathbf{t}_{t}^{(1)}\right|-\mu t_{N}^{(1)} \leq 0$, where $\mu$ is the limiting friction coefficient; if the contact is sliding, then the tangential traction is given by $\left|\mathbf{t}_{t}^{(1)}\right|=\mu t_{N}^{(1)}$. Thus, 
the traction at the contact surface is given by $\mathbf{t}_{c}^{(1)}=t_{N}^{(1)} \mathbf{n}_{c}^{(1)}+\mathbf{t}_{t}^{(1)}$. Tangential contact conditions due to friction are imposed by introducing a slip function $\mathbf{u}_{t}$, defined as

$$
\mathbf{u}_{t}(\mathbf{x})=\left(\mathbf{u}^{(1)}(\mathbf{x})-\mathbf{u}^{(2)}(\overline{\mathbf{y}}(\mathbf{x}))\right)-\left[\left(\mathbf{u}^{(1)}(\mathbf{x})-\mathbf{u}^{(2)}(\overline{\mathbf{y}}(\mathbf{x}))\right) \cdot \mathbf{n}_{c}^{(1)}(\mathbf{x})\right] \mathbf{n}_{c}^{(1)}(\mathbf{x})
$$

which is a local measure of relative tangential displacement between contacting surfaces. In addition to the Kuhn-Tucker optimality conditions, frictional contact requires that $\left|\mathbf{u}_{t}\right| \geq 0$, $\phi_{t} \leq 0$, and $\left|\mathbf{u}_{t}\right| \phi_{t}=0$. In summary, the contact boundary condition is given by

$$
\boldsymbol{\sigma}^{(i)} \cdot \mathbf{n}_{c}^{(i)}=\mathbf{t}_{c}^{(i)} \text { on } \Gamma_{c}^{(i)} \forall t \in \tau
$$

Contact boundary condition for the temperature field is given by

$$
\left(-k_{T} \boldsymbol{\nabla}_{x} T\right) \cdot \mathbf{n}_{c}^{(i)}=q_{f}^{(i)}+q_{c}^{(i)} \text { on } \Gamma_{c}^{(i)} \forall t \in \tau,
$$

where $q_{f}^{(i)}$ is the heat flux due to frictional heating associated with particle $\Omega^{(i)}$, and $q_{c}^{(i)}$ is the heat flux necessary to impose ideal thermal contact. The local heat flux due to friction at a contact surface is given by

$$
q_{f}=\mathbf{t}_{t} \cdot \dot{\mathbf{u}}_{t}
$$

where $\dot{\mathbf{u}}_{t}$ is the local tangential slip rate given by the time derivative of Eq. (2.61). The heat flux is distributed between the contacting particles based on the relation

$$
q_{f}^{(1)}=\omega q_{f}, \quad q_{f}^{(2)}=(1-\omega) q_{f},
$$

where the partition function $\omega$ is given by

$$
\omega=\left(1+\sqrt{\frac{k_{T}^{(2)} c_{v}^{(2)} \rho^{(2)}}{k_{T}^{(1)} c_{v}^{(1)} \rho^{(1)}}}\right)^{-1}
$$


It is further assumed that heat transfer between the particles is ideal because thermal resistance is ignored for both stick and slip conditions; this assumption is mathematically expressed by

$$
T^{(1)}(\mathbf{x}, t)=T^{(2)}(\overline{\mathbf{y}}(\mathbf{x}), t) \text { on } \Gamma_{c}^{(1)} \cap \Gamma_{c}^{(2)} \forall t \in \tau,
$$

implying that temperature is continuous across a contact interface. The heat flux required to impose this condition is given by

$$
q_{c}^{(1)}=-q_{c}^{(2)}=R\left(T^{(1)}(\mathbf{x}, t)-T^{(2)}(\overline{\mathbf{y}}(\mathbf{x}), t)\right)
$$

where $R$ is the contact conductance. For perfect thermal contact $R \rightarrow \infty$. This constraint is numerically imposed in a similar manner to the kinematic contact constraints with $R$ interpreted as a thermal penalty parameter $p e^{t h}$ and the thermal gap function defined as

$$
g^{t h}(\mathbf{x})=T^{(1)}(\mathbf{x})-T^{(2)}(\overline{\mathbf{y}}(\mathbf{x}))
$$

Finally, because this study focuses on the plane strain response of the particle ensemble for simplicity, all out-of-plane deformations are forced to vanish.

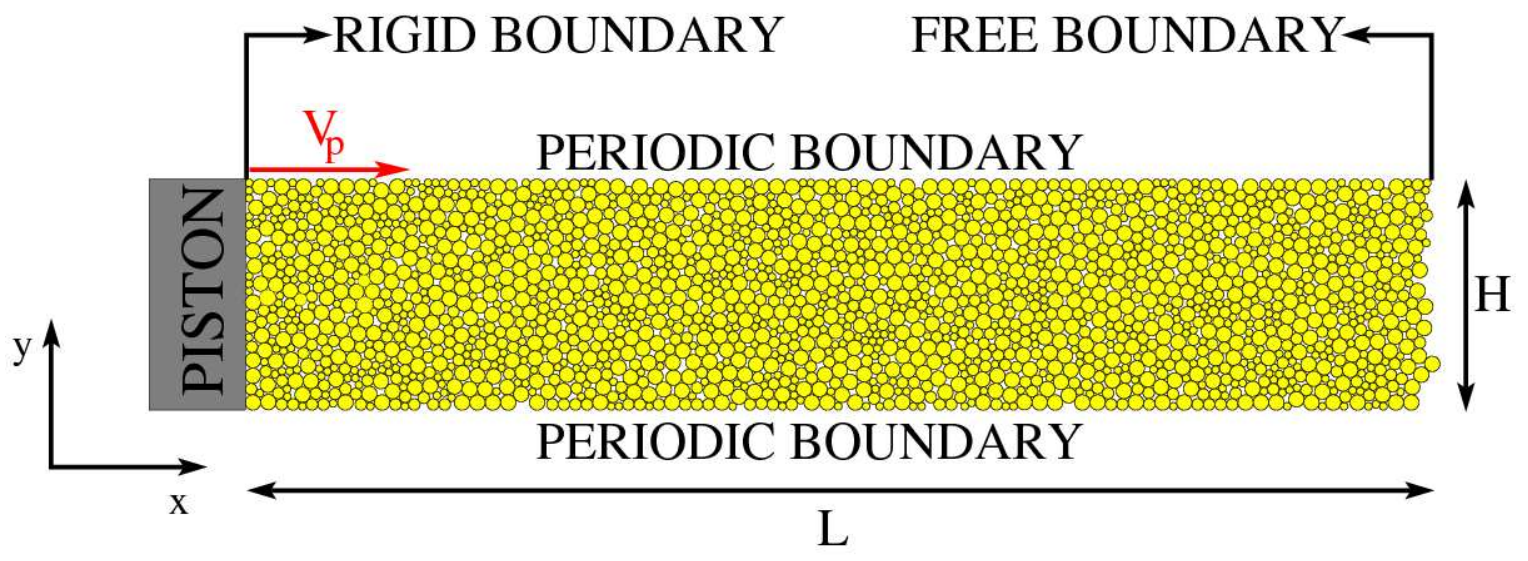

Figure 2.4: Global boundary conditions on the particle ensemble.

A representative initial configuration for the particle ensemble under consideration is shown in Fig. 2.4. The $\mathrm{x}$ and $\mathrm{y}$ components of the displacement and velocity field vectors are denoted 
as $\left[u_{x}, u_{y}\right]$ and $\left[v_{x}, v_{y}\right]$, respectively. At time $\mathrm{t}=0$, the particles are at rest and the piston is given an initial velocity $v_{p}$ in the positive x-direction. Free boundary conditions exist on the right boundary whereas contact boundary conditions are enforced on the left boundary between the rigid moving piston and the particles. Periodic boundary conditions are imposed on the top and bottom boundary. Mathematically, these boundary conditions are given by

$$
\begin{aligned}
& u_{x}(x, y=0, t)=u_{x}(x, y=H, t), \\
& u_{y}(x, y=0, t)=u_{y}(x, y=H, t), \\
& v_{x}(x, y=0, t)=v_{x}(x, y=H, t), \\
& v_{y}(x, y=0, t)=v_{y}(x, y=H, t) .
\end{aligned}
$$




\section{Chapter 3}

\section{Numerical Method}

The numerical techniques used to simulate piston induced deformation waves in $2 \mathrm{D}$ particulate solids is formulated in this chapter. Analytical solutions to the governing equations cannot be obtained for most impact problems due to complicated constitutive relations and complex interaction forces between solids. Consequently, finite element techniques are used to solve the differential forms of the governing equations numerically. In this study, simulations are performed using a combined finite and discrete element method that is well-suited for problems involving discontinua. This method uses the finite element method (FEM) to numerically integrate the time-dependent, 2-D conservation principles and viscoplastic flow rule governing deformation of individual particles, and uses the discrete element method (DEM) to account for interactions between grains.

The conservative equations of motion presented in the previous chapter represent the strong form of the IBVP for continuous media. To numerically solve the problem using the FEM, the variational form of the IBVP must be utilized. Since the finite element algorithm is Lagrangian and the system is closed, the continuity equation is trivially satisfied. In this chapter, the discretized form of the momentum and energy equations are first derived from their respective continuous

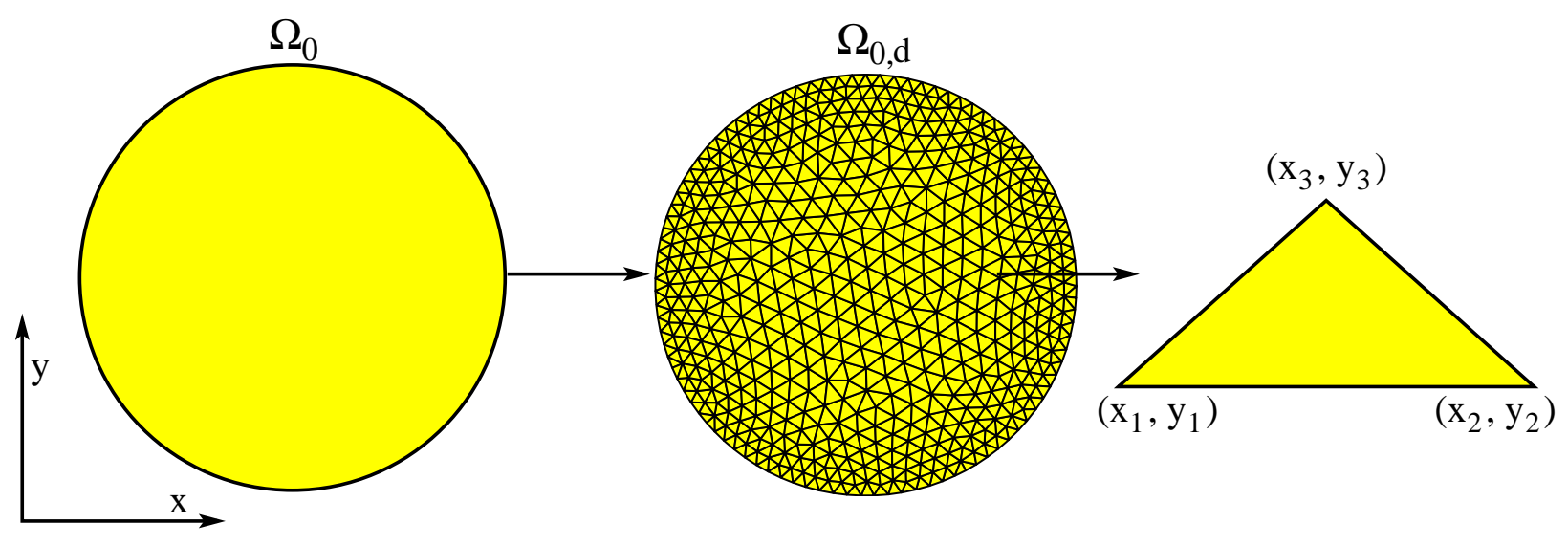

Figure 3.1: Discretization of a representative particle $\Omega_{0}$. 
forms in Section 3.1. Then, the numerical strategies used to evaluate each term of the discretized momentum and energy equations are presented in Section 3.2 and 3.3, respectively.

\subsection{Discretization of the Governing Equations}

Prior to deriving the discrete forms of the equations of motion, a few key features of the discretization procedure are presented. Figure 3.1 shows an example of a representative particle $\Omega_{0}$ discretized using three-node constant strain triangular (CST) finite elements. Here, $\Omega_{0, d}$ represents the discretized form of $\Omega_{0}$. The discretization process transforms the continuous domain into discrete points or nodes. Solution variables such as displacement, velocity and temperature are obtained directly at the nodes and are estimated at non-nodal locations using interpolation techniques, such as locally defined shape functions. Material state variables such as density and stress are estimated at interpolation points within the elements. In this study, discretization is established in the initial configuration using CST finite elements to interpolate the initial geometry in terms of material points $\mathbf{X}_{k}$ defining the initial position of the element nodes as,

$$
\mathbf{X}=\sum_{k=1}^{3} N_{k} \mathbf{X}_{k}
$$

where $N_{k}$ are the standard shape functions associated with node $k$. During motion, the nodes and elements are permanently attached to the material points with which they were initially associated. The subsequent motion is fully described in terms of the current position $\mathbf{x}$ and the displacement $\mathbf{u}$ of the nodes as

$$
\mathbf{x}=\sum_{k=1}^{3} N_{k} \mathbf{x}_{k}, \quad \mathbf{u}=\sum_{k=1}^{3} N_{k} \mathbf{u}_{k},
$$

respectively. The corresponding interpolation for the velocity and temperature field is given by

$$
\mathbf{v}=\sum_{k=1}^{3} N_{k} \mathbf{v}_{k}, \quad T=\sum_{k=1}^{3} N_{k} T_{k}
$$

respectively. As seen later, in deriving the variational form of the governing equations it is common to define and use virtual variables, such as, the virtual velocity $\delta \mathbf{v}$ and the virtual temperature 
$\delta T$, which are interpolated as

$$
\delta \mathbf{v}=\sum_{k=1}^{3} N_{k} \delta \mathbf{v}_{k}, \quad \delta T=\sum_{k=1}^{3} N_{k} \delta T_{k}
$$

respectively. The virtual velocity and temperature fields represent imaginary, arbitrary continuous functions that satisfy the prescribed displacement and temperature boundary conditions, that is, $\delta \mathbf{v}=\mathbf{0}$ on $\Gamma_{u}$ and $\delta T=0$ on $\Gamma_{T}$. Finally, the deformation gradient is interpolated over an element by differentiating Eq. (3.2) with respect to the initial configuration to give

$$
\mathbf{F}=\sum_{k=1}^{3} \mathbf{x}_{k} \otimes \nabla_{0} N_{k}
$$

The above relations can be used to interpolate any spatial or material solution variable at any point in a finite element. For example, using the definition of $\mathbf{d}$ given by Eq. (2.4), the discretized real and virtual rate of deformation is given as

$$
\mathbf{d}=\frac{1}{2} \sum_{k=1}^{3}\left(\mathbf{v}_{k} \otimes \nabla_{x} N_{k}+\nabla_{x} N_{k} \otimes \mathbf{v}_{k}\right),
$$

and

$$
\delta \mathbf{d}=\frac{1}{2} \sum_{k=1}^{3}\left(\delta \mathbf{v}_{k} \otimes \nabla_{x} N_{k}+\nabla_{x} N_{k} \otimes \delta \mathbf{v}_{k}\right)
$$

respectively.

Key features of the CST finite element are now described. The geometry of the 3-node CST finite element, as shown in Fig. 3.1, is specified by the location of its three corner nodes on the 2D Cartesian $\{x-y\}$ plane. The nodes are labelled 1,2, 3 while traversing the sides in counterclockwise fashion. The location of the corners is defined by their current Cartesian coordinates: $\left\{x_{i}, y_{i}\right\}_{i=1,2,3}$. The element has six degrees of freedom, defined by the six nodal displacement components $\left\{u_{x, i}, u_{y, i}\right\}_{i=1,2,3}$.

In Cartesian coordinates, the shape functions for a CST finite element in the current config- 
uration are given by

$$
\left[\begin{array}{c}
N_{1} \\
N_{2} \\
N_{3}
\end{array}\right]=\frac{1}{2 A}\left[\begin{array}{ccc}
\alpha_{1} & \beta_{1} & \gamma_{1} \\
\alpha_{2} & \beta_{2} & \gamma_{2} \\
\alpha_{3} & \beta_{3} & \gamma_{3}
\end{array}\right]\left[\begin{array}{l}
1 \\
x \\
y
\end{array}\right]
$$

where

$$
\left[\begin{array}{ccc}
\alpha_{1} & \beta_{1} & \gamma_{1} \\
\alpha_{2} & \beta_{2} & \gamma_{2} \\
\alpha_{3} & \beta_{3} & \gamma_{3}
\end{array}\right]=\left[\begin{array}{lll}
x_{2} y_{3}-y_{2} x_{3} & y_{2}-y_{3} & x_{3}-x_{2} \\
y_{1} x_{3}-x_{1} y_{3} & y_{3}-y_{1} & x_{1}-x_{3} \\
x_{1} y_{2}-y_{1} x_{2} & y_{1}-y_{2} & x_{2}-x_{1}
\end{array}\right],
$$

where $A$ is the current area of the finite element. In the initial configuration, the shape functions are given by

$$
\left[\begin{array}{c}
N_{1} \\
N_{2} \\
N_{3}
\end{array}\right]=\frac{1}{2 A_{0}}\left[\begin{array}{ccc}
\alpha_{1}^{\prime} & \beta_{1}^{\prime} & \gamma_{1}^{\prime} \\
\alpha_{2}^{\prime} & \beta_{2}^{\prime} & \gamma_{2}^{\prime} \\
\alpha_{3}^{\prime} & \beta_{3}^{\prime} & \gamma_{3}^{\prime}
\end{array}\right]\left[\begin{array}{c}
1 \\
X \\
Y
\end{array}\right]
$$

where

$$
\left[\begin{array}{ccc}
\alpha_{1}^{\prime} & \beta_{1}^{\prime} & \gamma_{1}^{\prime} \\
\alpha_{2}^{\prime} & \beta_{2}^{\prime} & \gamma_{2}^{\prime} \\
\alpha_{3}^{\prime} & \beta_{3}^{\prime} & \gamma_{3}^{\prime}
\end{array}\right]=\left[\begin{array}{ccc}
X_{2} Y_{3}-Y_{2} X_{3} & Y_{2}-Y_{3} & X_{3}-X_{2} \\
Y_{1} X_{3}-X_{1} Y_{3} & Y_{3}-Y_{1} & X_{1}-X_{3} \\
X_{1} Y_{2}-Y_{1} X_{2} & Y_{1}-Y_{2} & X_{2}-X_{1}
\end{array}\right],
$$

where $\left[X_{i}, Y_{i}\right]_{i=1,2,3}$ are the initial Cartesian coordinates of the nodes of the finite element and $A_{0}$ is its initial area.

\subsubsection{Weak Form of the Momentum Equation}

In the present work, the continuous momentum equation is simplified to create a system of algebraic equations. To this end, the principle of virtual work is invoked. If $\delta \mathbf{v}$ denotes an arbitrary virtual velocity from the current position of an arbitrary particle $\Omega$, then, in a state of equilibrium, the work done by residual forces during this virtual motion is obtained by taking the contraction product of Eq. (2.2) with $\delta \mathbf{v}$ and integrating the result over $\Omega$ :

$$
\delta \mathcal{W}=\int_{\bar{\Omega}}\left(\nabla_{x} \cdot \boldsymbol{\sigma}-\rho \ddot{\mathbf{u}}\right) \cdot \delta \mathbf{v} d \bar{\Omega}=0,
$$


where the notation $\delta \mathcal{W}$ is used to symbolically represent the result. Here, body forces are ignored as they are inconsequential compared to the impact induced deformation forces predicted in this study. Using the property $\boldsymbol{\nabla}_{x} \cdot(\boldsymbol{\sigma} \delta \mathbf{v})=\left(\boldsymbol{\nabla}_{x} \cdot \boldsymbol{\sigma}\right) \cdot \delta \mathbf{v}+\boldsymbol{\sigma}: \boldsymbol{\nabla}_{x} \delta \mathbf{v}$ together with the divergence theorem, Eq. (3.12) can be simplified to give

$$
\delta \mathcal{W}=\int_{\Omega}\left(\boldsymbol{\sigma}: \nabla_{x} \delta \mathbf{v}+\rho \ddot{\mathbf{u}} \cdot \delta \mathbf{v}\right) d \Omega-\int_{\Gamma} \mathbf{n} \cdot \boldsymbol{\sigma} \delta \mathbf{v} d \Gamma=0
$$

where $\mathbf{n}$ is the outward unit normal to $\Gamma$. The gradient of $\delta \mathbf{v}$ is, by definition, the virtual velocity gradient $\delta$ l. Also, using the definition of the traction vector, that is, $\mathbf{t}=\boldsymbol{\sigma} \cdot \mathbf{n}$, and the symmetry of $\boldsymbol{\sigma}$ to rewrite $\mathbf{n} \cdot \boldsymbol{\sigma} \delta \mathbf{v}$ as $\delta \mathbf{v} \cdot \mathbf{t}$, the above equation becomes

$$
\delta \mathcal{W}=\int_{\Omega}(\boldsymbol{\sigma}: \delta \mathbf{l}+\rho \ddot{\mathbf{u}} \cdot \delta \mathbf{v}) d \Omega-\int_{\Gamma} \mathbf{t} \cdot \delta \mathbf{v} d \Gamma=0
$$

Expressing $\delta \mathbf{l}$ in terms of the symmetric virtual deformation rate tensor $\delta \mathbf{d}$ and the anti-symmetric virtual spin tensor $\delta \mathbf{w}$ as $\delta \mathbf{l}=\delta \mathbf{d}+\delta \mathbf{w}$, gives the virtual work as

$$
\delta \mathcal{W}=\int_{\Omega}(\boldsymbol{\sigma}: \delta \mathbf{d}+\rho \ddot{\mathbf{u}} \cdot \delta \mathbf{v}) d \Omega-\int_{\Gamma} \mathbf{t} \cdot \delta \mathbf{v} d \Gamma=0
$$

where $\boldsymbol{\sigma}: \delta \mathbf{w}=0$ is used to obtain the above equation. Recognizing that $\Gamma_{\sigma}=\Phi$, and that $\delta \mathbf{v}=\mathbf{0}$ on $\Gamma_{u}$, the above equation can be further simplified to give the final form of the fundamental scalar equation to be used for finite element discretization as

$$
\delta \mathcal{W}=\int_{\Omega}(\boldsymbol{\sigma}: \delta \mathbf{d}+\rho \ddot{\mathbf{u}} \cdot \delta \mathbf{v}) d \Omega-\int_{\Gamma_{c}} \mathbf{t}_{c} \cdot \delta \mathbf{v} d \Gamma_{c}=0,
$$

subject to the weak form of the initial conditions given by

$$
\begin{gathered}
\int_{\bar{\Omega}}\left[\left.\mathbf{u}\right|_{t=0}-\mathbf{u}_{0}\right] \cdot \delta \mathbf{v} d \bar{\Omega}=0, \\
\int_{\bar{\Omega}}\left[\left.\mathbf{v}\right|_{t=0}-\mathbf{v}_{0}\right] \cdot \delta \mathbf{v} d \bar{\Omega}=0 .
\end{gathered}
$$


The last term in Eq. (3.16) corresponds to the contact virtual work on $\Gamma_{c}$. The variational form appropriate for $\mathcal{N}_{p}$ particle contact problem is obtained by summation of all the individual weak forms given by

$$
\delta \mathcal{W}=\sum_{i=1}^{\mathcal{N}_{p}} \delta \mathcal{W}^{(i)}=\delta \mathcal{W}_{m}+\delta \mathcal{W}_{i}+\delta \mathcal{W}_{e}=0
$$

where

$$
\delta \mathcal{W}_{m}=\sum_{i=1}^{\mathcal{N}_{p}}\left(\int_{\Omega^{(i)}}\left(\rho^{(i)} \ddot{\mathbf{u}}^{(i)} \cdot \delta \mathbf{v}^{(i)}\right) d \Omega^{(i)}\right),
$$

and

$$
\delta \mathcal{W}_{i}=\sum_{i=1}^{\mathcal{N}_{p}}\left(\int_{\Omega^{(i)}}\left(\boldsymbol{\sigma}^{(i)}: \delta \mathbf{d}^{(i)}\right) d \Omega^{(i)}\right)
$$

and

$$
\delta \mathcal{W}_{e}=\sum_{i=1}^{\mathcal{N}_{p}}\left(\int_{\Gamma_{c}^{(i)}}-\left(\mathbf{t}_{c}^{(i)} \cdot \delta \mathbf{v}^{(i)}\right) d \Gamma_{c}^{(i)}\right) .
$$

Here, $\delta \mathcal{W}_{m}, \delta \mathcal{W}_{i}$ and $\delta \mathcal{W}_{e}$ represent the total virtual work due to inertial terms, internal stresses and external loads (i.e. contact forces), respectively.

The goal of the discretization procedure is to express Eq. (3.18) in the finite element form

$$
\mathcal{M U}-\mathcal{F}_{i}-\mathcal{F}_{e}=0
$$

where $\mathcal{U}\left[\mathcal{N}_{n}, 3\right]$ is the global nodal displacement matrix, $\mathcal{M}\left[\mathcal{N}_{n}, \mathcal{N}_{n}\right]$ is the global nodal mass matrix, $\mathcal{F}_{e}\left[\mathcal{N}_{n}, 3\right]$ and $\mathcal{F}_{i}\left[\mathcal{N}_{n}, 3\right]$ are the global nodal external and internal force vectors that represent finite element discretizations of $\delta \mathcal{W}_{e}$ and $\delta \mathcal{W}_{i}$, respectively. Here, $\mathcal{N}_{n}$ represents the total number on nodes in the discretized system and the notation $\left[\mathcal{N}_{n}, \mathcal{N}_{n}\right]$ is used to denote a matrix of $\mathcal{N}_{n}$ rows and $\mathcal{N}_{n}$ columns. To this end, consider the contribution to $\delta \mathcal{W}$ caused by a single virtual nodal velocity $\delta \mathbf{v}_{k}$ occurring at a typical node $k$ of finite element $\Delta^{(j)}$. First, substituting for $\mathbf{u}$ and $\delta \mathbf{v}$ using Eqs. (3.2) and (3.3) in the inertial virtual work relation and simplifying the result gives

$$
\delta \mathcal{W}_{m}^{(j)}=\int_{\Delta^{(j)}}\left(N_{l} \rho N_{k}\right)\left(\ddot{\mathbf{u}}_{l} \cdot \delta \mathbf{v}_{k}\right) d v
$$


where $l, k=1,2,3$ are nodal indices. Second, substituting for $\delta \mathbf{v}$ and $\delta \mathbf{d}$ in the internal virtual work relation and using the property $\boldsymbol{\sigma}:\left(\delta \mathbf{v}_{k} \otimes \nabla_{x} N_{k}\right)=\delta \mathbf{v}_{k} \cdot \boldsymbol{\sigma} \boldsymbol{\nabla}_{x} N_{k}$ gives

$$
\delta \mathcal{W}_{i}^{(j)}=\int_{\Delta^{(j)}} \delta \mathbf{v}_{k} \cdot\left(\boldsymbol{\sigma} \nabla_{x} N_{k}\right) d v
$$

Third, substituting for $\delta \mathbf{v}$ in the external virtual work relation gives

$$
\delta \mathcal{W}_{e}^{(j)}=\int_{\Delta_{c}^{(j)}}-\mathbf{t}_{c} \cdot\left(N_{k} \delta \mathbf{v}_{k}\right) d a
$$

where $\Delta_{c}^{(j)}$ represents the contact boundary of $\Delta^{(j)}$. Recognizing that the virtual nodal velocities are arbitrary and therefore independent of integration, $\delta \mathbf{v}_{k}$ can be eliminated from Eqs. (3.23), (3.24) and (3.25) to give the contribution of node $k$ of element $\Delta^{(j)}$ to the element mass, internal and external force matrices as

$$
[\mathcal{M}]_{k l}^{(j)}=\int_{\Delta^{(j)}} 2 \rho N_{k} \delta_{k l} N_{l} d v, \quad\left[\mathcal{F}_{i}\right]_{k}^{(j)}=\int_{\Delta^{(j)}} \boldsymbol{\sigma} \nabla_{x} N_{k} d v, \quad\left[\mathcal{F}_{e}\right]_{k}^{(j)}=\int_{\Delta_{c}^{(j)}} N_{k} \mathbf{t}_{\mathbf{c}} d a
$$

respectively, where $\delta_{k l}$ is the Kronecker delta. The element mass matrix in the above equation corresponds to a lumped mass matrix, where discrete element mass is allocated to finite element nodes thereby eliminating the need to assemble large stiffness and mass matrices that result in complex equations. Here, the factor 2 in the first part of the above equation represents the scaling factor required to obtain the lumped mass matrix from the consistent mass matrix. The global mass and force matrices are obtained by summation over all the finite elements as

$$
\mathcal{M}=\sum_{i=1}^{\mathcal{N}_{p}}\left(\sum_{j=1}^{\mathcal{N}_{e}(i)}[\mathcal{M}]^{(j)}\right), \quad \mathcal{F}_{i}=\sum_{i=1}^{\mathcal{N}_{p}}\left(\sum_{j=1}^{\mathcal{N}_{e}(i)}\left[\mathcal{F}_{i}\right]^{(j)}\right), \quad \mathcal{F}_{e}=\sum_{i=1}^{\mathcal{N}_{p}}\left(\sum_{j=1}^{\mathcal{N}_{e}(i)}\left[\mathcal{F}_{e}\right]^{(j)}\right)
$$

where $\mathcal{N}_{e}(i)$ is the number of finite elements used to discretize $\Omega^{(i)}$. 


\subsubsection{Weak Form of the Temperature Equation}

For an arbitrary particle $\Omega$, the discretized form of the temperature equation is obtained by taking the product between Eq. (2.54) and the virtual temperature field $\delta T$ and integrating over its volume to give

$$
\int_{\bar{\Omega}}\left(\rho c_{v} \frac{d T}{d t}+\nabla_{x} \cdot \mathbf{q}-\dot{Q}^{e}-\dot{Q}^{i}\right) \delta T d \bar{\Omega}=0
$$

Here, it is assumed that there is no internal heat generation, that is, $r=0$. Using the property $\boldsymbol{\nabla}_{x} \cdot(\mathbf{q} \delta T)=\left(\boldsymbol{\nabla}_{x} \cdot \mathbf{q}\right) \delta T+\mathbf{q}: \boldsymbol{\nabla}_{x} \delta T$ together with the divergence theorem in the above expression gives

$$
\int_{\Omega}\left(\rho c_{v} \frac{d T}{d t}-\dot{Q}^{e}-\dot{Q}^{i}\right) \delta T d \Omega-\int_{\Omega}\left(\mathbf{q}: \nabla_{x} \delta T\right) d \Omega=-\int_{\Gamma_{c}}\left(q_{f}+q_{c}\right) \delta T d \Gamma_{c}
$$

where $\delta T=0$ on $\Gamma_{T}, \bar{q}=0$ on $\Gamma_{q}$, and the contact boundary condition given by Eq. (2.63) have been used to obtain the above equation.

The purpose of the discretization procedure is to reduce the variational form of the temperature evolution equation to the finite element form given as

$$
\mathcal{M}_{c} \dot{\mathcal{T}}+\mathcal{K}_{c} \mathcal{T}=\mathcal{F}_{c}
$$

where $\mathcal{M}_{c}\left[\mathcal{N}_{n}, \mathcal{N}_{n}\right]$ is the lumped thermal capacitance matrix, $\mathcal{K}_{c}\left[\mathcal{N}_{n}, \mathcal{N}_{n}\right]$ is the stiffness matrix, $\mathcal{F}_{c}\left[\mathcal{N}_{n}, 1\right]$ is the thermal force matrix and $\mathcal{T}\left[\mathcal{N}_{n}, 1\right]$ is the nodal temperature matrix. To this end, consider the contribution of single virtual temperature $\delta T_{k}$ of a particular node $k$ of finite element $\Delta^{(j)}$ to Eq. (3.29), given by

$$
\begin{array}{r}
\int_{\Delta^{(j)}}\left(\rho c_{v} \frac{d}{d t}\left(N_{l} T_{l}\right)\right) N_{k} \delta T_{k} d v+\int_{\Delta^{(j)}}\left(k_{T} \nabla_{x}\left(N_{l} T_{l}\right): \nabla_{x} N_{k} \delta T_{k}\right) d v \\
=\int_{\Delta^{(j)}}\left(\dot{Q}^{e}+\dot{Q}^{i}\right) N_{k} \delta T_{k} d v-\int_{\Delta_{c}^{(j)}}\left(q_{f}+q_{c}\right) N_{k} \delta T_{k} d a .
\end{array}
$$

Here, Fourier's law has been used to replace q, and Eq. (3.4) to replace $T$ with its discrete counter- 
part. Recognizing that virtual temperature is arbitrary and therefore independent of integration, $\delta T_{k}$ can be eliminated from Eq. (3.31). Rearranging terms, the above expression can be simplified to give

$$
\begin{array}{r}
\frac{d T_{l}}{d t} \int_{\Delta^{(j)}}\left(\rho c_{v} N_{l} N_{k}\right) d v+T_{l} \int_{\Delta^{(j)}}\left(k_{T} \nabla_{x} N_{l}: \nabla_{x} N_{k}\right) d v \\
=\int_{\Delta^{(j)}}\left(\dot{Q}^{e}+\dot{Q}^{i}\right) N_{k} d v-\int_{\Delta_{c}^{(j)}}\left(q_{f}+q_{c}\right) N_{k} d a .
\end{array}
$$

Using the above equation, the contribution of a particular node $k$ of element $\Delta^{(j)}$ to the element thermal capacitance, stiffness and force matrices is obtained as

$$
\begin{array}{r}
{\left[\mathcal{M}_{c}\right]_{k l}^{(j)}=\int_{\Delta^{(j)}} 2 \rho c_{v} N_{k} \delta_{k l} N_{l} d v, \quad\left[\mathcal{K}_{c}\right]_{k l}^{(j)}=\int_{\Delta^{(j)}} k_{T} \nabla_{x} N_{k}: \nabla_{x} N_{l} d v} \\
{\left[\mathcal{F}_{c}\right]_{k}^{(j)}=\int_{\Delta^{(j)}}\left(\dot{Q}^{e}+\dot{Q}^{i}\right) N_{k} d v-\int_{\Delta_{c}^{(j)}}\left(q_{f}+q_{c}\right) N_{k} d a .}
\end{array}
$$

The global capacitance, stiffness and force matrices are obtained by summation over all the finite elements as

$$
\mathcal{M}_{c}=\sum_{i=1}^{\mathcal{N}_{p}}\left(\sum_{j=1}^{\mathcal{N}_{e}(i)}\left[\mathcal{M}_{c}\right]^{(j)}\right), \quad \mathcal{K}_{c}=\sum_{i=1}^{\mathcal{N}_{p}}\left(\sum_{j=1}^{\mathcal{N}_{e}(i)}\left[\mathcal{K}_{c}\right]^{(j)}\right), \quad \mathcal{F}_{c}=\sum_{i=1}^{\mathcal{N}_{p}}\left(\sum_{j=1}^{\mathcal{N}_{e}(i)}\left[\mathcal{F}_{c}\right]^{(j)}\right)
$$

A temporally second-order accurate, explicit numerical technique is used to integrate the finite element equations of motion for the nodal displacements $\mathcal{U}(t)$ and nodal temperatures $\mathcal{T}(t)$. At an arbitrary time step $t_{n}>0$, the finite element equations of motion and temperature evolution equation can be expressed as

$$
\mathcal{M} \ddot{\mathcal{U}}_{n}=\mathcal{F}_{i, n}+\mathcal{F}_{e, n}, \quad \mathcal{M}_{c} \dot{\mathcal{T}}_{n}+\mathcal{K}_{c, n} \mathcal{T}_{n}=\mathcal{F}_{c, n}
$$

The external load vector $\mathcal{F}_{e, n}$ is known at the beginning of the time step, whereas the internal load vector $\mathcal{F}_{i, n}$, the thermal load matrix $\mathcal{F}_{c, n}$ and the stiffness matrix $\mathcal{K}_{c, n}$ are functions of $\mathcal{U}_{n}$ and $\mathcal{T}_{n}$, which are the known nodal displacement and temperature fields at the indicated time step. 
The central difference integration method is used to calculate displacements and velocities at the next time increment:

$$
\mathcal{V}_{n+\frac{1}{2}}=\mathcal{V}_{n-\frac{1}{2}}+\Delta t \ddot{\mathcal{U}}_{n}, \quad \mathcal{U}_{n+1}=\mathcal{U}_{n}+\Delta t \mathcal{V}_{n+\frac{1}{2}}
$$

where $\Delta t$ is the time interval calculated at the beginning of each indicated time increment. Here, $\mathcal{V}\left[\mathcal{N}_{n}, 3\right]$ is the global nodal velocity matrix at the indicated time step. Special consideration must be given to the application of initial conditions $(n=0)$ since the velocity term $\mathcal{V}_{n-\frac{1}{2}}$ in the above equation is undefined at the initial state. For this case, Eq. (3.36) is modified to include the initial condition:

$$
\mathcal{V}_{\frac{1}{2}}=\mathcal{V}_{0}-\frac{\Delta t_{0}}{2} \ddot{\mathcal{U}}_{0}
$$

The velocities at the time increment $t_{n+1}$ are determined as:

$$
\mathcal{V}_{n+1}=\mathcal{V}_{n+\frac{1}{2}}+\frac{\Delta t}{2} \ddot{\mathcal{U}}_{n+1}
$$

Finally, the nodal temperatures are calculated using a forward-difference integration technique with respect to time. Using the known temperatures at time step $t_{n}$ and the calculated temperature rate vector:

$$
\mathcal{T}_{n+1}=\mathcal{T}_{n}+\Delta t \dot{\mathcal{T}}_{n}
$$

Numerical stability requires that each time interval $\Delta t$ be less than the time needed for an elastic wave to traverse the smallest finite element within the domain. The longitudinal elastic wave speed for a material having Young's modulus $E=24$ Gpa and density $\rho_{0}=1903 \mathrm{~kg} / \mathrm{m}^{3}$, which are representative values used in this study, is given by $\sqrt{E / \rho_{0}}=3.5513 \times 10^{3} \mathrm{~m} / \mathrm{s}$. For a representative finite element size of $\Delta h \approx 3 \mu \mathrm{m}$, we have that $\Delta t_{\text {crit }}=\Delta h / \sqrt{E / \rho_{0}}=0.84476 \mathrm{~ns}$. A value of $\Delta t=0.01 \mathrm{~ns}<\Delta t_{\text {crit }}$ was used for all simulations performed in this study. 


\subsection{Discretized Momentum Equation}

The numerical strategies used to evaluate the internal and external force matrices are described in this section in detail. At a given time $t_{n}$, the element lumped mass matrix for an arbitrary CST finite element $\Delta^{(j)}$ is given by

$$
[\mathcal{M}]^{(j)}=\frac{\rho A}{3}\left[\begin{array}{lll}
1 & 0 & 0 \\
0 & 1 & 0 \\
0 & 0 & 1
\end{array}\right] .
$$

The final form of the global lumped mass matrix is obtained by summing over all the finite elements as given by Eq. (3.27).

\subsubsection{Internal Force Matrix}

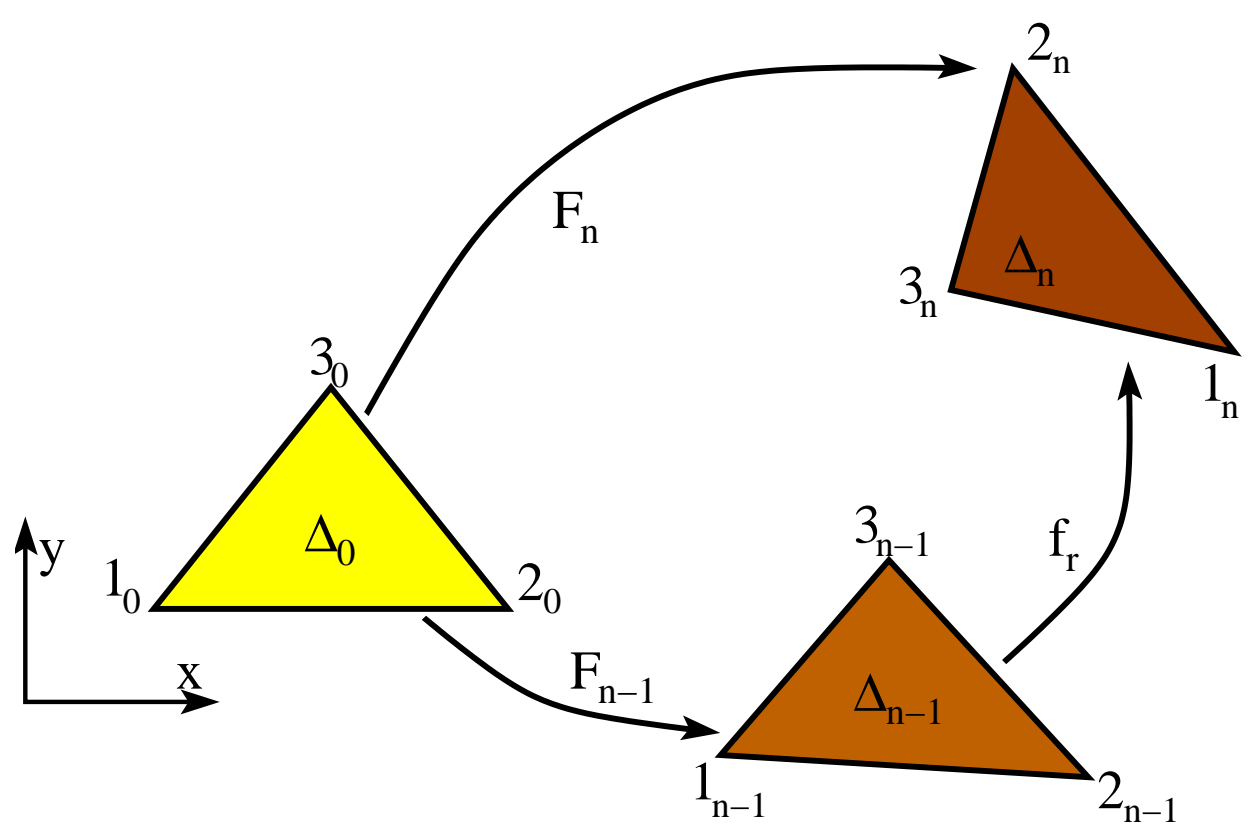

Figure 3.2: Incremental motion of a finite element between time $t_{n-1}$ and $t_{n}$.

Consider the motion of an arbitrary finite element between time $t_{n-1}$ and $t_{n}$ as shown in Fig. 3.2. To estimate $\mathcal{F}_{i, n}$, which implicitly depends on the stress field $\boldsymbol{\tau}_{n}$ through $\mathcal{U}_{n}$, a local timestepping procedure is applied to the constitutive relations. At time $t_{n-1}$, data for $\mathbf{b}_{e, n-1}$ and $\mathbf{F}_{n-1}$ 
are given. At time $t_{n}, \mathbf{F}_{n}$ is obtained from $\mathcal{U}_{n}$ using Eqs. (3.5) and (3.10) as

$$
\mathbf{F}_{n}=\left[\begin{array}{ccc}
\frac{\left(x_{1} Y_{23}+x_{2} Y_{31}+x_{3} Y_{12}\right)}{2 A_{0}} & \frac{\left(x_{1} X_{32}+x_{2} X_{13}+x_{3} X_{21}\right)}{2 A_{0}} & 0 \\
\frac{\left(y_{1} Y_{23}+y_{2} Y_{31}+y_{3} Y_{12}\right)}{2 A_{0}} & \frac{\left(y_{1} X_{32}+y_{2} X_{13}+y_{3} X_{21}\right)}{2 A_{0}} & 0 \\
0 & 0 & 1
\end{array}\right]
$$

where $X_{k l} \equiv X_{k}-X_{l}$ and $Y_{k l} \equiv Y_{k}-Y_{l}$. In this study, plane strain conditions are automatically enforced by setting the principal out of plane component of $\mathbf{F}$ to unity and the out of plane deviatoric components to zero.

The solution procedure is initiated by assuming that the plastic variables remain frozen in time during the current increment and that the incremental deformation is entirely elastic. Subsequently, a trial value for $\mathbf{b}_{e}$ and $\epsilon_{p}$ is computed as $\mathbf{b}_{e}^{\text {trial }}=\mathbf{f}_{r} \mathbf{b}_{e, n-1} \mathbf{f}_{r}^{T}$ and $\epsilon_{p}^{\text {trial }}=\epsilon_{p, n-1}$, respectively, where $\mathbf{f}_{r}=\mathbf{F}_{n} \mathbf{F}_{n-1}^{-1}$ is the incremental deformation gradient. Using Eq. (2.11), $\mathbf{b}_{e}^{\text {trial }}$ is decomposed into its eigenvalues $\boldsymbol{\lambda}_{e}^{\text {trial }}$ and eigenvectors $\mathbf{n}^{\text {trial }}$. Subsequently, using Eq. (2.45), the principal volumetric and deviatoric components of $\boldsymbol{\tau}^{\text {trial }}$ are evaluated as

$$
p^{\text {trial }}=\frac{1}{J_{n}}\left(k \ln \left(J_{n}\right)-3 k \alpha\left(\hat{T}_{n}-T_{0}\right) \frac{\left(1-\ln \left(J_{n}\right)\right)}{J_{n}}\right),
$$

and

$$
\overline{\boldsymbol{\tau}}_{i i}^{\text {trial }}=2 G \ln \left(\frac{\lambda_{e, i}^{\text {trial }}}{J_{n}^{1 / 3}}\right),
$$

respectively, where $\hat{T}_{n}$ is the mass weighted average temperature of the finite element at time $t_{n}$. Because the plastic correction, if needed, is performed at a fixed principal axis, $\mathbf{n}=\mathbf{n}^{\text {trial }}$. Because all volumetric strains are elastic $J_{n}$ is unaffected by the plastic correction procedure. In addition, the plastic correction procedure is performed at a fixed temperature $\hat{T}_{n}$. Both these conditions together imply that $p_{n}=p^{\text {trial }}$.

Based on $\bar{\tau}^{\text {trial }}$ the yield criterion is estimated as

$$
\phi^{\text {trial }}=\sqrt{\overline{\boldsymbol{\tau}}^{\text {trial }}: \overline{\boldsymbol{\tau}}^{\text {trial }}}-\sqrt{\frac{2}{3}}\left(\tau_{0}+h \epsilon_{p}^{\text {trial }}\right) .
$$


If $\phi^{\text {trial }} \leq 0$ then the strain increment is entirely elastic and the trial values are correct, that is, $\mathbf{b}_{e, n}=\mathbf{b}_{e}^{\text {trial }}$ and $\epsilon_{p, n}=\epsilon_{p}^{\text {trial }}$. Consequently $\boldsymbol{\tau}_{n}=\boldsymbol{\tau}^{\text {trial }}$. Otherwise, if $\phi^{\text {trial }}>0$, the evolution equations for the plastic variables Eq. (2.34) are simultaneously integrated using an implicit, forward Euler method to estimate $\mathbf{b}_{e, n}$ and $\epsilon_{p, n}$. Here, the solution is first obtained for the true elastic stretches as $\lambda_{e, i}=\exp \left(-\Delta \epsilon \xi_{i}\right) \lambda_{e, i}^{\text {trial }}$, where

$$
\xi_{i}=\frac{\bar{\tau}_{\text {ii }}^{\text {trial }}}{\sqrt{\overline{\boldsymbol{\tau}}^{\text {trial }}: \overline{\boldsymbol{\tau}}^{\text {trial }}}}
$$

and $\Delta \epsilon$, which is the incremental consistency parameter defined as $\Delta \epsilon=\int_{t_{n-1}}^{t_{n}} \dot{\epsilon} d t$, is obtained as a part of the solution procedure. Subsequently, the elastic left Cauchy-Green tensor is updated as

$$
\mathbf{b}_{e, n}=\sum_{i=1}^{3}\left(\lambda_{e, i}\right)^{2} \mathbf{n}_{i} \otimes \mathbf{n}_{i}
$$

the von Mises equivalent plastic strain is updated as

$$
\epsilon_{p, n}=\epsilon_{p, n-1}+\sqrt{\frac{2}{3}} \Delta \epsilon
$$

and the true deviatoric stresses are updated as

$$
\bar{\tau}_{i i}=\bar{\tau}_{i i}^{\text {trial }}-2 G \Delta \epsilon \xi_{i}
$$

For linear isotropic hardening (i.e., for constant $h$ ), a closed form solution can be obtained for $\Delta \epsilon$. Using Eq. (2.38), for this case, the modified yield criterion given by

$$
\bar{\phi}_{n}=\sqrt{\frac{3}{2} \overline{\boldsymbol{\tau}}_{n}: \overline{\boldsymbol{\tau}}_{n}}-\left(\tau_{0}+h \epsilon_{p, n}\right)-\sqrt{\frac{2}{3}}(\gamma \dot{\epsilon})=0
$$

First, approximating $\dot{\epsilon}$ as $\Delta \epsilon / \Delta t$, and then using this result along with Eqs. (3.47) and (3.48) in 
Eq. (3.49), gives the closed form solution for $\Delta \epsilon$ as

$$
\Delta \epsilon=\frac{1}{2 G}\left\langle\frac{\phi^{\text {trial }}}{1+\left(h+\frac{\gamma}{\Delta t}\right) /(3 G)}\right\rangle .
$$

Once the stress state for each finite element has been numerically updated, the equivalent nodal internal force acting on each finite element is computed using Eq. (3.26) as

$$
\left[\mathcal{F}_{i, n}\right]_{k, p=1,2,3}^{(j)}=\sum_{q=1}^{3} \int_{\Delta^{(j)}} \boldsymbol{\sigma}_{n, p q} \frac{\partial N_{k}}{\partial \mathbf{x}_{q}} d v
$$

where $\boldsymbol{\sigma}_{n}=\boldsymbol{\tau}_{n} / J_{n}$. The above expression can be expanded to give

$$
\left[\mathcal{F}_{i, n}\right]_{k, p=1,2,3}^{(j)}=\int_{\Delta^{(j)}}\left(\boldsymbol{\sigma}_{n, p 1} \frac{\partial N_{k}}{\partial x}+\boldsymbol{\sigma}_{n, p 2} \frac{\partial N_{k}}{\partial y}\right) d v
$$

Using Eqs. (3.10) and (3.11), the above equation can be expressed in matrix notation as

$$
\left[\mathcal{F}_{i, n}\right]^{(j)}=\frac{1}{2}\left[\begin{array}{cccc}
\left(\boldsymbol{\sigma}_{n, 11}\left(y_{2}-y_{3}\right)+\boldsymbol{\sigma}_{n, 12}\left(x_{3}-x_{2}\right)\right) & \left(\boldsymbol{\sigma}_{n, 21}\left(y_{2}-y_{3}\right)+\boldsymbol{\sigma}_{n, 22}\left(x_{3}-x_{2}\right)\right) & 0 \\
\left(\boldsymbol{\sigma}_{n, 11}\left(y_{3}-y_{1}\right)+\boldsymbol{\sigma}_{n, 12}\left(x_{1}-x_{3}\right)\right) & \left(\boldsymbol{\sigma}_{n, 21}\left(y_{3}-y_{1}\right)+\boldsymbol{\sigma}_{n, 22}\left(x_{1}-x_{3}\right)\right) & 0 \\
\left(\boldsymbol{\sigma}_{n, 11}\left(y_{1}-y_{2}\right)+\boldsymbol{\sigma}_{n, 12}\left(x_{2}-x_{1}\right)\right) & \left(\boldsymbol{\sigma}_{n, 21}\left(y_{1}-y_{2}\right)+\boldsymbol{\sigma}_{n, 22}\left(x_{2}-x_{1}\right)\right) & 0
\end{array}\right] .
$$

This procedure is followed for all the finite elements for each time increment and the resulting forces on the nodes are assembled in a matrix form to give the internal force matrix $\mathcal{F}_{i, n}$.

\subsubsection{External Force Matrix}

Contact interaction between discrete elements generally occurs along irregular contact surfaces and involves a number of penetrating finite elements that increases with contact pressure. The contact problem is further complicated by the inclusion of friction dynamics within the penetration zone. Frictional phenomena is usually resolved in computational mechanics using a variational formulation of contact combined with both a force-displacement law and a stick-slip condition. 
Penalty based methods are a popular choice for contact/impact problems because they are both algorithmically simpler and computationally less expensive than Lagrangian or augmented Lagrangian methods. The penalty method works by penalizing violations of the kinematic constraint by associating rather large energies with them. This is done by first establishing a suitable measure to estimate the extent of interpenetration between particles, such as the gap function $g$ given by Eq. (2.60), and then penalizing this measure by multiplying it with the penalty parameter. This method has the advantage of removing the constraints explicitly from the variational formulation by optimizing the problem with respect to only one solution variable, that is, the displacement field $\mathbf{u}$. Formally stated, the problem is equivalent to making the global potential energy functional $\Pi(\mathbf{u})$ stationary subject to the contact constraints. For the simplest case, that is, for a frictional contact problem of two arbitrary linear elastic particles, the functional can be defined as

$$
\bar{\Pi}(\mathbf{u}, \mathbf{p e})=\Pi^{c}\left(\mathbf{u}, p e^{n}, p e^{t}\right)+\sum_{i=1}^{2} \Pi^{(i)}(\mathbf{u}),
$$

where $\Pi^{(i)}(\mathbf{u})$ is the total potential energy functional and $\Pi^{c}\left(\mathbf{u}, p e^{n}, p e^{t}\right)$ is the contribution due to contact to the global functional. Here, $p e^{n}$ and $p e^{t}$ are the normal and tangential penalty parameters, respectively. The quantity $\Pi^{(i)}(\mathbf{u})$, the total potential energy associated with the particle $\Omega^{(i)}$ is given by

$$
\Pi^{(i)}(\mathbf{u})=\int_{\Omega^{(i)}} \frac{1}{2} \boldsymbol{\sigma}^{(i)}: \boldsymbol{\epsilon}^{(i)} d \Omega^{(i)}
$$

where $\boldsymbol{\epsilon}$ is the small strain tensor. The contact functional for the frictional contact problem is given by

$$
\Pi^{c}\left(\mathbf{u}, p e^{n}, p e^{t}\right)=\int_{\Gamma_{c}}\left(\frac{p e^{n}}{2}<g>^{2}+\frac{p e^{t}}{2} \mathbf{u}_{t} \cdot \mathbf{u}_{t}\right) d \Gamma_{c}
$$

The solution to this problem is obtained by minimizing $\bar{\Pi}\left(\mathbf{u}, p e^{n}, p e^{t}\right)$ by rendering it stationary with respect to variations $\delta \mathbf{v}$ of $\mathbf{u}$ given by

$$
0=\left.\frac{d}{d \alpha}\right|_{\alpha=0} \Pi^{c}\left(\mathbf{u}+\alpha \delta \mathbf{v}, p e^{n}, p e^{t}\right)+\left.\sum_{i=1}^{2} \frac{d}{d \alpha}\right|_{\alpha=0} \Pi^{(i)}(\mathbf{u}+\alpha \delta \mathbf{v})
$$


where $\alpha$ is a scalar and $\delta \mathbf{v}$ is the virtual velocity field. Using Eqs. (3.55) and (3.56) the first and the second term on the right hand side of Eq. (3.57) can be simplified as

$$
\left.\frac{d}{d \alpha}\right|_{\alpha=0} \Pi^{c}\left(\mathbf{u}+\alpha \delta \mathbf{v}, p e^{n}, p e^{t}\right)=\left(\int_{\Gamma_{c}} p e^{n}<g>\delta g+p e^{t} \mathbf{u}_{t} \cdot \delta \mathbf{u}_{t}\right) d \Gamma_{c},
$$

and

$$
\left.\sum_{i=1}^{2} \frac{d}{d \alpha}\right|_{\alpha=0} \Pi^{(i)}(\mathbf{u}+\alpha \delta \mathbf{v})=\sum_{i=1}^{2}\left(\int_{\Omega^{(i)}} \boldsymbol{\sigma}^{(i)}: \delta \mathbf{d}^{(i)} d \Omega^{(i)}\right)
$$

respectively. Equation (3.59) represents the internal virtual work analogous to $\delta W_{i}$ shown in Eq. (3.20). Equation (3.58) represents the external virtual work analogous to $\delta W_{e}$ given in Eq. (3.21), which can be formally stated as

$$
\sum_{i=1}^{2}\left(\int_{\Gamma_{c}^{(i)}}-\left(\mathbf{t}_{c}^{(i)} \cdot \delta \mathbf{v}^{(i)}\right) d \Gamma_{c}^{(i)}\right)=\left(\int_{\Gamma_{c}} p e^{n}<g>\delta g+p e^{t} \mathbf{u}_{t} \cdot \delta \mathbf{u}_{t}\right) d \Gamma_{c}
$$

Recognizing that the contact tractions on the two particles are equal and opposite, that is, $\mathbf{t}_{\mathbf{c}}{ }^{(1)}(\mathbf{x})=-\mathbf{t}_{\mathbf{c}}{ }^{(2)}(\overline{\mathbf{y}}(\mathbf{x}))$, the above equation can be expressed as

$$
\begin{array}{r}
\int_{\Gamma_{c}^{(1)}}\left(t_{N}^{(1)} \mathbf{n}_{c}^{(1)}+\mathbf{t}_{t}^{(1)}\right) \cdot\left(\delta \mathbf{v}^{(1)}(\mathbf{x})-\delta \mathbf{v}^{(2)}(\overline{\mathbf{y}}(\mathbf{x}))\right) d \Gamma_{c}^{(1)} \\
=\int_{\Gamma_{c}^{(1)}}\left(p e^{n}<g>\delta g+p e^{t} \mathbf{u}_{t}^{(1)} \cdot \delta \mathbf{u}_{t}^{(1)}\right) d \Gamma_{c}^{(1)}
\end{array}
$$

Using the definitions of the gap function and the slip function given by Eqs. (2.60) and (2.61), respectively, their derivatives can be expressed as

$$
\delta g=\left.\frac{d}{d \alpha}\right|_{\alpha=0} g(\mathbf{u}+\alpha \delta \mathbf{v})=\mathbf{n}_{c}^{(1)} \cdot\left(\delta \mathbf{v}^{(1)}(\mathbf{x})-\delta \mathbf{v}^{(2)}(\overline{\mathbf{y}}(\mathbf{x}))\right)
$$

and

$$
\begin{aligned}
\delta \mathbf{u}_{t}^{(1)}=\left.\frac{d}{d \alpha}\right|_{\alpha=0} \mathbf{u}_{t}^{(1)} & (\mathbf{u}+\alpha \delta \mathbf{v})=\left(\delta \mathbf{v}^{(1)}(\mathbf{x})-\delta \mathbf{v}^{(2)}(\overline{\mathbf{y}}(\mathbf{x}))\right) \\
- & \left(\left(\delta \mathbf{v}^{(1)}(\mathbf{x})-\delta \mathbf{v}^{(2)}(\overline{\mathbf{y}}(\mathbf{x}))\right) \cdot \mathbf{n}_{c}^{(1)}\right) \mathbf{n}_{c}^{(1)}
\end{aligned}
$$


respectively. Finally, substituting the above results in the right hand side of Eq. (3.61) and comparing with the left hand side gives expressions for the normal and tangential traction as

$$
t_{N}^{(1)}=p e^{n}<g>, \quad \mathbf{t}_{t}^{(1)}=p e^{t} \mathbf{u}_{t}^{(1)},
$$

respectively. Here, $\mathbf{u}_{t}^{(1)} \cdot \mathbf{n}_{c}^{(1)}=0$ is used to obtain the second part of the above expression.

The numerical strategies used to evaluate the external force vector $\mathcal{F}_{e}$ are now described. To evaluate the normal forces arising due to contact, given by Eq. (3.64), a modified gap potential is used rather than the standard gap function given by Eq. (2.60). The modified gap potential is not restricted to the contact boundary but it is defined for all points located within the penetration region. This enables contact between discrete elements to be handled in an edge-to-edge or edge-tosurface manner, rather than node-to-node manner which is known to lead to numerical distortion of the strain fields close to the boundary. This method also results in a more realistic distribution of contact forces.

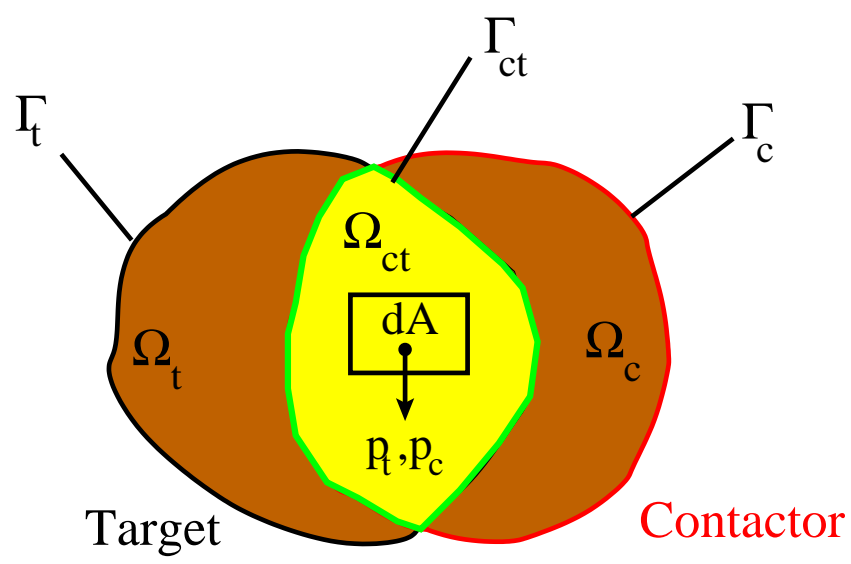

(a)

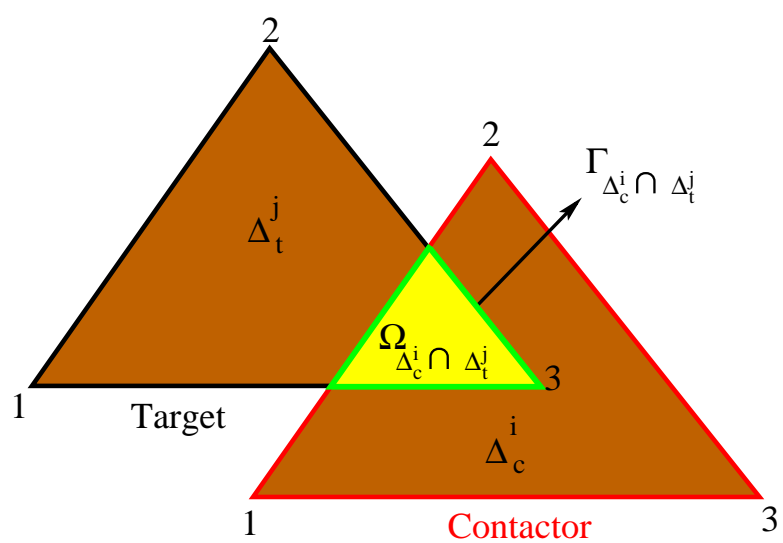

(b)

Figure 3.3: Schematic of the discrete element contact model: (a) interaction of target and contactor discrete elements; (b) interaction of target and contactor finite elements.

Consider a pair of discrete elements in contact in the current configuration, as shown in Fig. 3.3(a). Here, one of the discrete elements is denoted as $\Omega_{c}$ called the contactor element and the other as $\Omega_{t}$ called the target element. The contactor and target element overlap over an area $\Omega_{c t}$, which is bounded by $\Gamma_{c t}$. For a differential element $d A$ within the penetration region, the 
differential contact force prescribed by the 2-D penalty based distributed contact force method is given by

$$
d \mathbf{f}_{N}=p e^{n}\left[\boldsymbol{\nabla}_{x} \Phi_{c}\left(\mathbf{p}_{c}\right)-\nabla_{x} \Phi_{t}\left(\mathbf{p}_{t}\right)\right] d A,
$$

where $\Phi_{c}$ and $\Phi_{t}$ are the modified conservative gap potential fields defined on the contactor and target elements, respectively, and $\mathbf{p}_{c}$ and $\mathbf{p}_{t}$ are global position vectors of the contactor and target element within $d A$. This differential contact force can be decomposed into a component resulting from the target penetrating the contactor $d \mathbf{f}_{c}$ and a component resulting from the contactor penetrating the target $d \mathbf{f}_{t}$ as $d \mathbf{f}_{n}=-d \mathbf{f}_{t}+d \mathbf{f}_{c}$, where $d \mathbf{f}_{t}$ and $d \mathbf{f}_{c}$ are given by

$$
d \mathbf{f}_{t}=-p e^{n} \nabla_{x} \Phi_{c}\left(\mathbf{p}_{c}\right) d A, \quad d \mathbf{f}_{c}=-p e^{n} \nabla_{x} \Phi_{t}\left(\mathbf{p}_{t}\right) d A
$$

The net force is obtained by integrating the differential force over $\Omega_{c t}$ as

$$
\mathbf{f}_{N}=p e^{n} \int_{\Omega_{c t}}\left[\nabla_{x} \Phi_{c}-\nabla_{x} \Phi_{t}\right] d A=p e^{n} \int_{\Gamma_{c t}} \mathbf{n}_{c t}\left[\Phi_{c}-\Phi_{t}\right] d \Gamma_{c t}
$$

where $\mathbf{n}_{c t}$ is the outward unit normal to $\Gamma_{c t}$. Green's theorem is used in this equation to express the surface integral as a line integral. The contactor particle $\left(\Omega_{c}\right)$, containing $n$ finite elements, and the target particle $\left(\Omega_{t}\right)$, containing $m$ finite elements, may be represented by the union of their finite elements $\bigcup_{i=1}^{n} \Delta_{c}{ }^{i}$ and $\bigcup_{j=1}^{m} \Delta_{t}{ }^{j}$, respectively. Similarly, the potentials $\Phi_{c}$ and $\Phi_{t}$ may be represented by the sum of individual finite element potentials. Thus $\mathbf{f}_{N}$ can also be expressed as

$$
\mathbf{f}_{N}=p e^{n} \sum_{i=1}^{n} \sum_{j=1}^{m} \int_{\Gamma_{\Delta_{c}^{i} \cap \Delta_{t}^{j}}} \mathbf{n}_{\Delta_{c}^{i} \cap \Delta_{t}^{j}}\left[\Phi_{c}^{i}-\Phi_{t}^{j}\right] d \Gamma_{\Delta_{c}^{i} \cap \Delta_{t}^{j}} .
$$

Here, $\Phi_{c}^{i}$ and $\Phi_{t}^{j}$ are the potentials of the $i^{t h}$ contactor finite element and the $j^{\text {th }}$ target finite element and $\mathbf{n}_{\Delta_{c}^{i} \cap \Delta_{t}^{j}}$ is the outward unit normal to $\Gamma_{\Delta_{c}^{i} \cap \Delta_{t}^{j}}$, which represents the boundary of the interaction region between finite elements $\Delta_{c}^{i}$ and $\Delta_{t}^{j}$ as shown in Fig. 3.3(b).

The potential field on each finite element is prescribed so that its value is uniform on its boundary; consequently, the potential field is also uniform on the discrete element boundary. 


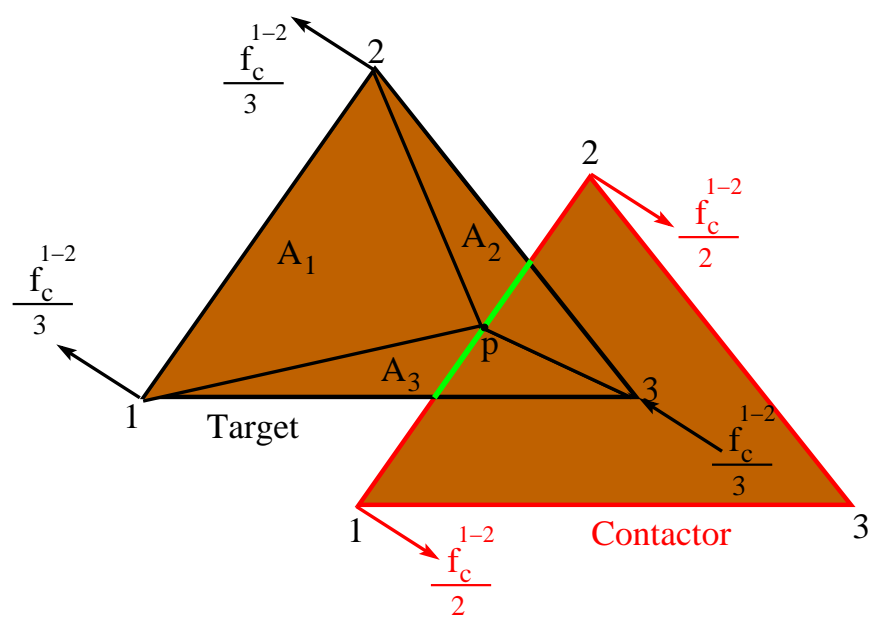

(a)

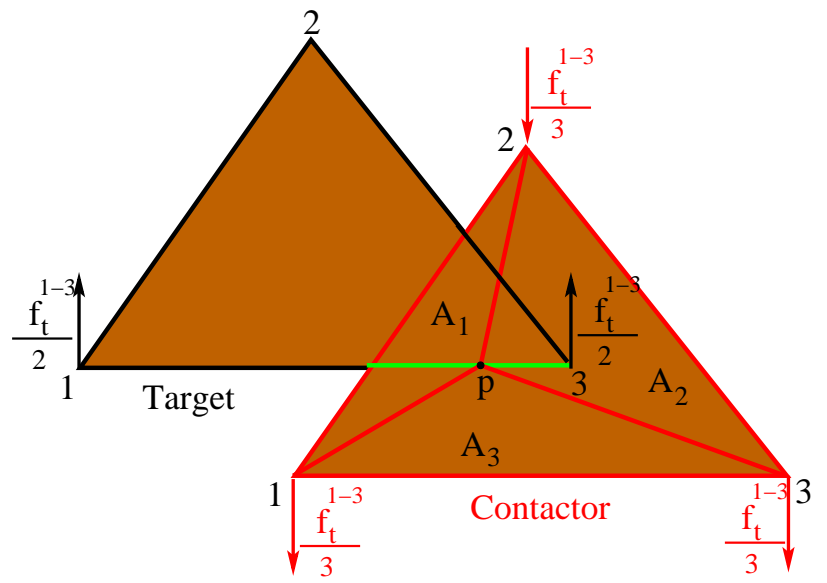

(b)

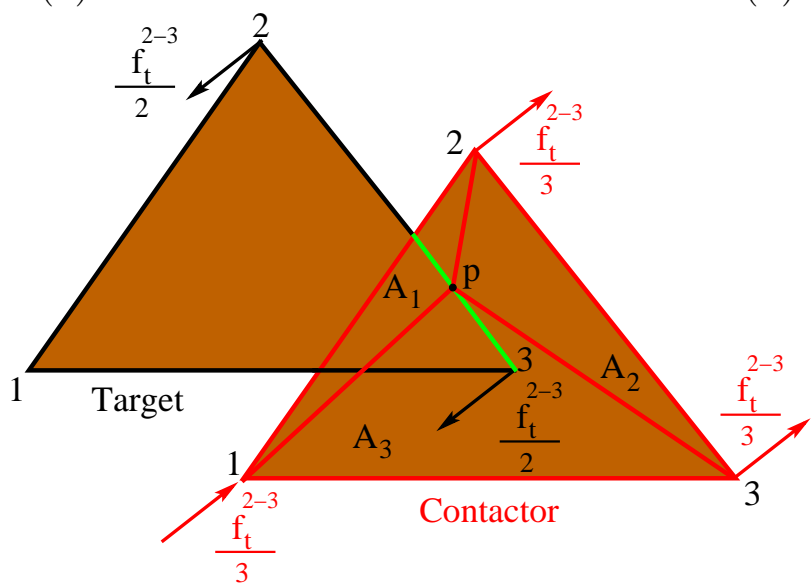

(c)

Figure 3.4: Schematic of the finite element contact model: contactor and target contact forces.

In this analysis, the potential field at a point $\mathbf{p}$ within a finite element is defined as $\Phi(\mathbf{p})=$ $\min (3 A 1 / A, 3 A 2 / A, 3 A 3 / A)$, where $A 1, A 2$, and $A 3$ are the areas of the finite element indicated in Fig. 3.4, with $A=A 1+A 2+A 3$. The net potential for each contactor and target finite element are then estimated by summing the potential $\Phi(\mathbf{p})$ at all points $\mathbf{p}$ of the contactor. For example, contribution to the net potential $\Phi_{t}$ from the contactor edge 1-2 intersecting the target finite element shown in Fig. 3.4(a) is estimated by integrating the individual potentials $\Phi(\mathbf{p})$ associated with point $\mathbf{p}$ over the edge as

$$
\Phi_{t}^{1-2}=\int_{1}^{2} \Phi(\mathbf{p}) d l
$$

For all points $\mathbf{p}$ on the edge 1-2 which do not lie inside the target finite element the potential 
associated with it is zero. The corresponding contactor force associated with the contactor edge $1-2$ is then given by

$$
\mathbf{f}_{c}^{1-2}=-p e^{n}\left[\mathbf{n}_{c}^{1-2} \Phi_{t}^{1-2}\right]
$$

where $\mathbf{n}_{c}^{1-2}$ is the outward normal to the contactor edge 1-2. This force is distributed to the nodes of the corresponding contactor edge and the target element as shown in Fig. 3.4(a). The same procedure is followed to estimate the contribution of the other edges towards the contactor and target force. The forces on the contactor element edges due to the contactor penetrating the target are then given by

$$
\mathbf{f}_{c}^{1-2}=-p e^{n}\left[\mathbf{n}_{c}^{1-2} \Phi_{t}^{1-2}\right], \quad \mathbf{f}_{c}^{2-3}=-p e^{n}\left[\mathbf{n}_{c}^{2-3} \Phi_{t}^{2-3}\right], \quad \mathbf{f}_{c}^{3-1}=-p e^{n}\left[\mathbf{n}_{c}^{3-1} \Phi_{t}^{3-1}\right],
$$

where $\mathbf{n}_{c}^{2-3}$ and $\mathbf{n}_{c}^{3-1}$ are the outward normals to edges 2-3 and 3-1 of the contactor, respectively. In the example shown $\mathbf{f}_{c}^{2-3}=\mathbf{f}_{c}^{3-1}=\mathbf{0}$. The corresponding force on each node of the target resulting from the contactor penetrating the target is given by

$$
\mathbf{f}_{t}^{1-2-3}=\frac{-1}{3}\left[\mathbf{f}_{c}^{1-2}+\mathbf{f}_{c}^{2-3}+\mathbf{f}_{c}^{3-1}\right]
$$

In the same manner, the forces on the target element edges due to the target penetrating the contactor are given by

$$
\mathbf{f}_{t}^{1-2}=-p e^{n}\left[\mathbf{n}_{t}^{1-2} \Phi_{c}^{1-2}\right], \quad \mathbf{f}_{t}^{2-3}=-p e^{n}\left[\mathbf{n}_{t}^{2-3} \Phi_{c}^{2-3}\right], \quad \mathbf{f}_{t}^{3-1}=-p e^{n}\left[\mathbf{n}_{t}^{3-1} \Phi_{c}^{3-1}\right],
$$

where $\mathbf{n}_{t}^{1-2}, \mathbf{n}_{t}^{2-3}$ and $\mathbf{n}_{t}^{3-1}$ are the outward normals to edges 1-2, 2-3 and 3-1 of the target respectively. In the example shown $\mathbf{f}_{t}^{1-2}=\mathbf{0}$. The corresponding force on each node of the contactor resulting from the target penetrating the contactor is given by

$$
\mathbf{f}_{c}^{1-2-3}=\frac{-1}{3}\left[\mathbf{f}_{t}^{1-2}+\mathbf{f}_{t}^{2-3}+\mathbf{f}_{t}^{3-1}\right]
$$


Finally, the element external normal force matrix for the contactor and target elements is given by

$$
\left[\mathcal{F}_{e, n}\right]^{(i)}=\left(\begin{array}{c}
\mathcal{F}_{e, n}^{1} \\
\mathcal{F}_{e, n}^{2} \\
\mathcal{F}_{e, n}^{3}
\end{array}\right)^{(i)}=\left(\begin{array}{c}
\frac{1}{2}\left[\mathbf{f}_{c}^{1-2}+\mathbf{f}_{c}^{3-1}\right]+\mathbf{f}_{c}^{1-2-3} \\
\frac{1}{2}\left[\mathbf{f}_{c}^{1-2}+\mathbf{f}_{c}^{2-3}\right]+\mathbf{f}_{c}^{1-2-3} \\
\frac{1}{2}\left[\mathbf{f}_{c}^{2-3}+\mathbf{f}_{c}^{3-1}\right]+\mathbf{f}_{c}^{1-2-3}
\end{array}\right)
$$

and

$$
\left[\mathcal{F}_{e, n}\right]^{(j)}=\left(\begin{array}{c}
\mathcal{F}_{e, n}^{1} \\
\mathcal{F}_{e, n}^{2} \\
\mathcal{F}_{e, n}^{3}
\end{array}\right)^{(j)}=\left(\begin{array}{c}
\frac{1}{2}\left[\mathbf{f}_{t}^{1-2}+\mathbf{f}_{t}^{3-1}\right]+\mathbf{f}_{t}^{1-2-3} \\
\frac{1}{2}\left[\mathbf{f}_{t}^{1-2}+\mathbf{f}_{t}^{2-3}\right]+\mathbf{f}_{t}^{1-2-3} \\
\frac{1}{2}\left[\mathbf{f}_{t}^{2-3}+\mathbf{f}_{t}^{3-1}\right]+\mathbf{f}_{t}^{1-2-3}
\end{array}\right),
$$

respectively. This procedure is followed for all such finite element interactions for each time increment and the resulting forces on the nodes are assembled in a matrix form to give the normal component of the external force vector $\mathcal{F}_{e, n}$. The numerical strategy used to estimate the tangential component of the external force matrix arising due to frictional tractions is now described.

- Friction model

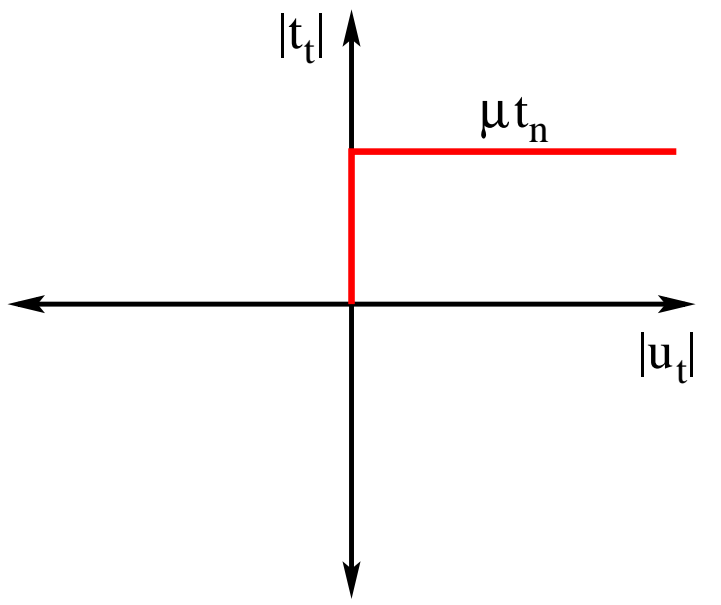

(a)

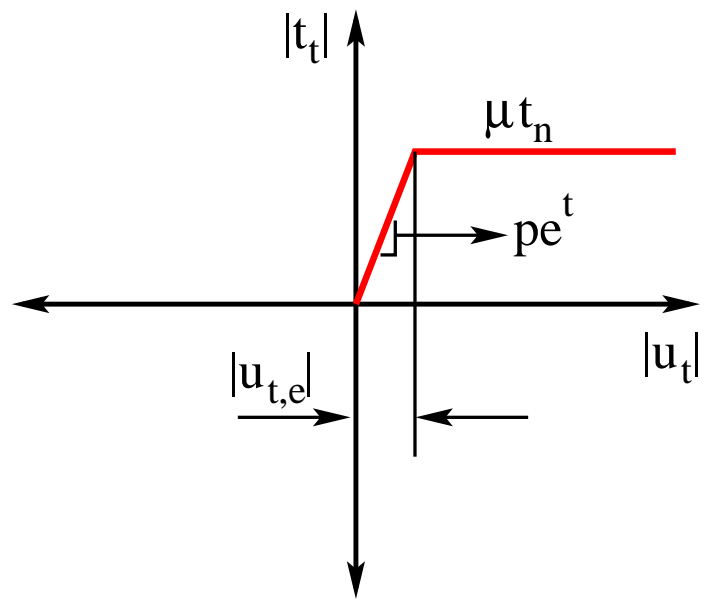

(b)

Figure 3.5: (a) Classical friction law (b) Regularized friction law.

The tangential component of the nodal external force matrix arising due to frictional traction at the contact interfaces is estimated using a penalty regularized Amontons-Coulomb law, which is also known as the plasticity theory of friction. Although the regularized friction law does not enforce the frictional constraints exactly, it is computationally simpler to implement than the 
classical friction law. The key idea of the elasto-plastic approach is a split of the tangential slip $\mathbf{u}_{t}$ into an elastic (stick or adhesion) part $\mathbf{u}_{t, e}$ and a plastic (slip) part $\mathbf{u}_{t, p}$ as

$$
\mathbf{u}_{t}=\mathbf{u}_{t, e}+\mathbf{u}_{t, p}
$$

In order to perform the additive decomposition of the tangential displacement into stick and slip, a slip criterion, analogous to a Von-Mises type yield criterion, must be introduced. To this end, a slip surface $\phi_{t}$ is introduced in the contact stress space where slip will occur. This is given by

$$
\phi_{t} \equiv\left|\mathbf{t}_{t}\right|-\mu t_{N}
$$

Also, a constitutive equation for the tangential traction analogous to a stress-strain relationship must be specified. This is given by

$$
\dot{\mathbf{t}}_{t}=p e^{t}\left(\dot{\mathbf{u}}_{t}-\dot{\mathbf{u}}_{t, p}\right)
$$

where $\dot{\mathbf{u}}_{t}$ is the total frictional slip rate given by the time derivative of Eq. (2.61) and $\dot{\mathbf{u}}_{t, p}$ is the plastic component of the frictional slip rate. The tangential penalty parameter is defined as the slope of the tangential traction-displacement line within the elastic domain. The classical Amontons-Coulomb law is recovered for $p e^{t} \rightarrow \infty$, as shown in Fig. 3.5. However, in practice, to avoid numerical instabilities, the tangential penalty parameter is assumed to scale as the inverse of the time increment $\Delta t$.

The system of equations is closed by assuming an evolution equation for the plastic frictional slip rate of the form

$$
\dot{\mathbf{u}}_{t, p}=\dot{\epsilon}_{t} \frac{\partial \phi_{t}}{\partial \mathbf{t}_{t}}=\dot{\epsilon}_{t} \frac{\mathbf{t}_{t}}{\left|\mathbf{t}_{t}\right|}, \text { where }\left\{\begin{array}{l}
\dot{\epsilon}_{t}=0, \text { if } \phi_{t}<0 \\
\dot{\epsilon}_{t} \geq 0, \text { if } \phi_{t}=0
\end{array}\right.
$$

Here, $\dot{\epsilon}_{t}$ is a frictional multiplier analogous to the plastic multiplier in the rate independent formu- 
lation of perfect plasticity. Given the data for $t_{N, n}$, which is the normal component of the external force matrix at time $t_{n}$, and the incremental frictional slip $\Delta \mathbf{u}_{t, n}$, which can be estimated from the displacement field $\mathbf{u}_{n}$, the frictional traction vector is estimated by applying a forward-difference integration technique with respect to time to Eq. (3.78). This is given by

$$
\mathbf{t}_{t, n}=\mathbf{t}_{t, n-1}+p e^{t}\left(\Delta \mathbf{u}_{t, n}-\Delta \mathbf{u}_{t, p}\right)
$$

where $\Delta \mathbf{u}_{t, p}$ is given by

$$
\Delta \mathbf{u}_{t, p}=\left(\frac{<\left|\mathbf{t}_{t}^{\text {trial }}\right|-\mu t_{N, n}>}{p e^{t}}\right) \frac{\mathbf{t}_{t}^{\text {trial }}}{\left|\mathbf{t}_{t}^{\text {trial }}\right|}
$$

Here, $\mathbf{t}_{t}^{\text {trial }}$ is the trial frictional traction vector estimated as

$$
\mathbf{t}_{t}^{\text {trial }}=\mathbf{t}_{t, n-1}+p e^{t}\left(\Delta \mathbf{u}_{t, n}\right) .
$$

This procedure is followed for all nodes on the contact boundary of each particle and the resulting forces on the nodes are assembled in a matrix form to give the tangential component of the external force vector. The total nodal external force matrix $\mathcal{F}_{e, n}$ is then simply given by the matrix sum of its normal and tangential components.

\section{- Contact Detection}

At any given time, prior knowledge about the pairs of discrete and finite elements in contact is required before external forces due to contact interaction can be estimated. This is accomplished by implementing a contact detection algorithm, whose goal is to generate a contact matrix which includes all the pairs of finite elements in contact. In the following discussion, key features of the contact detection algorithm used in this study are highlighted.

Figure 3.6 shows a snapshot of a typical particle ensemble, where multiple particles are in contact with each other. Finite elements on the boundary of each particle are shown in red. Because fracture is not modeled in this study, the particles themselves do not break up to form new particles. Hence, contact can only occur between finite elements on the boundary of the discrete 


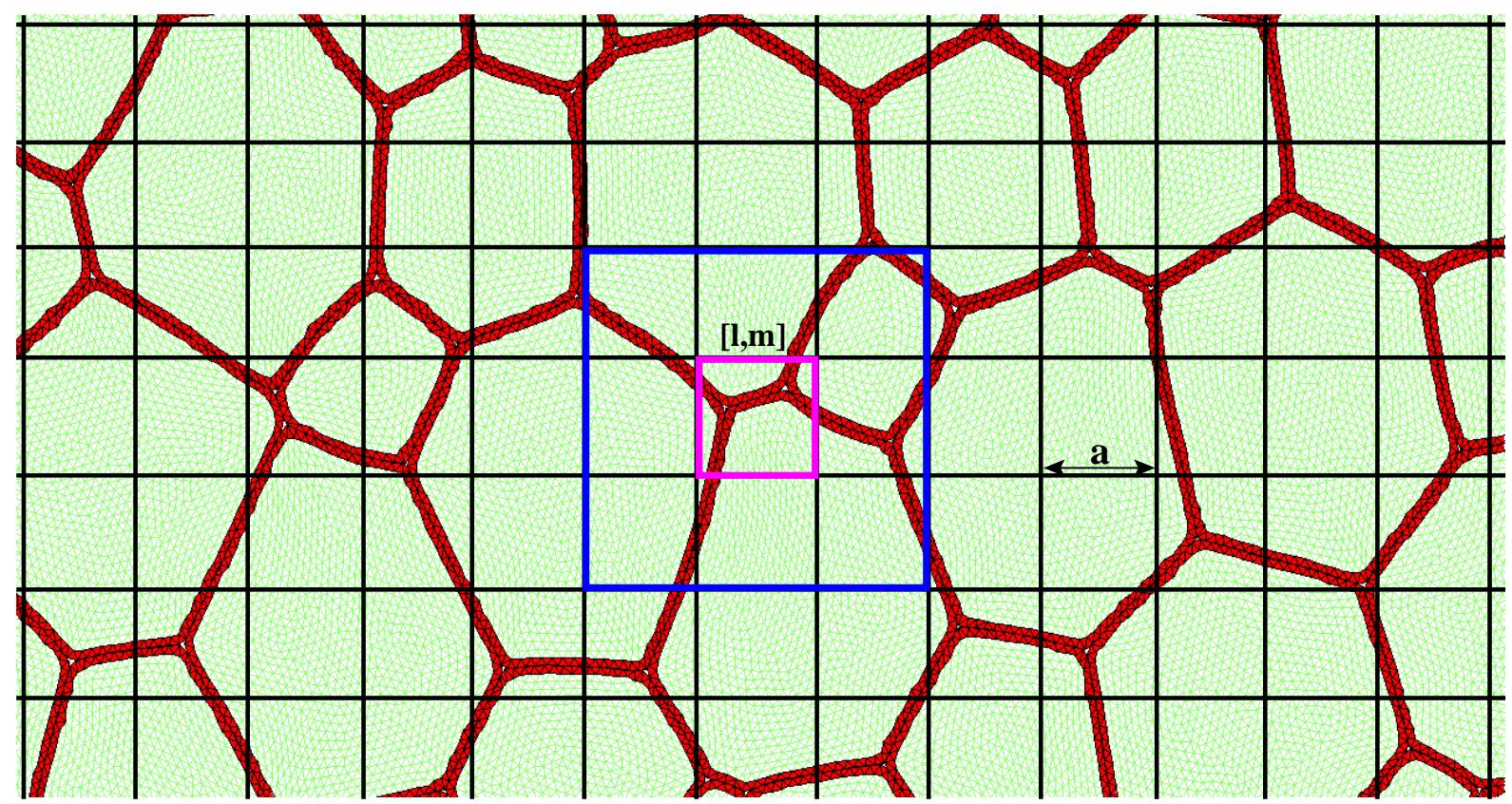

Figure 3.6: General layout for the contact detection algorithm.

elements, and therefore only these finite elements need to be considered for contact detection. In this study, contact detection is performed in two sequential stages. In the first stage, a nearest neighbor search is performed to sort finite elements based on their global positions inside the ensemble. To this end, the spatial domain is partitioned into a prescribed number of square cells of edge length $a$, where each cell is assigned a characteristic index $[l, m]$. Then, finite elements on the

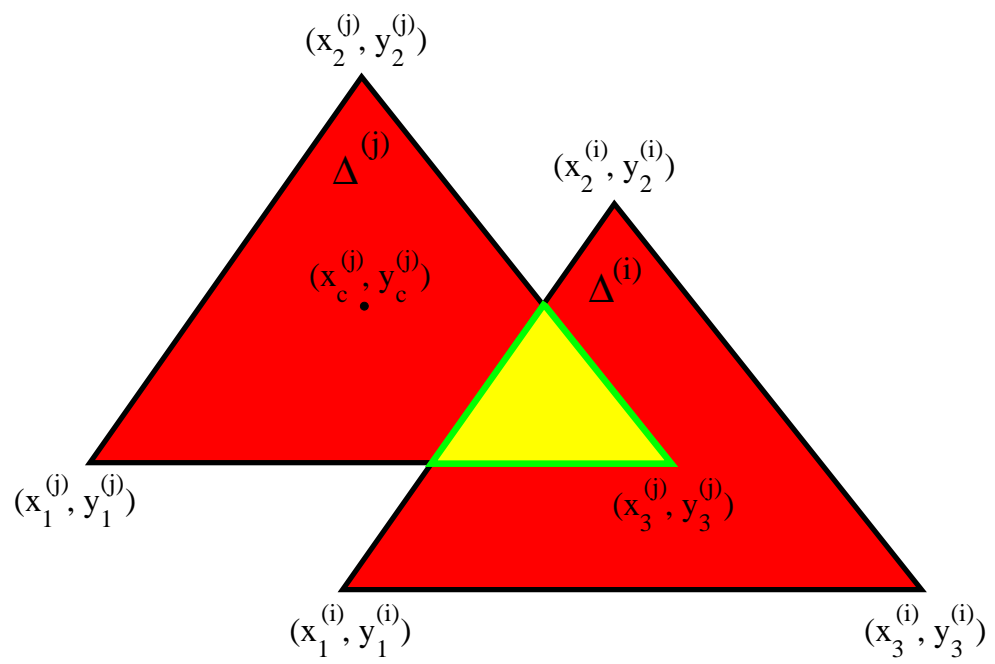

Figure 3.7: Contact between two neighbouring finite elements.

boundaries of all particles are placed within these cells based on the current global coordinates of 
their centroids. For example, a finite element $\Delta^{(j)}$, with the global coordinates of its centroid given by $\left[x_{c}^{(j)}, y_{c}^{(j)}\right]$, is indexed and placed in its respective cell as $l=\operatorname{int}\left(x_{c}^{(j)}\right)+1$ and $m=\operatorname{int}\left(y_{c}^{(j)}\right)+1$, where int $(\mathrm{x})$ is the integer function which gives the largest integer less than or equal to $\mathrm{x}$.

In the second stage, for each finite element inside the cell $[1, \mathrm{~m}]$, a triangle-triangle intersection test is performed with all finite elements within the cell as well as the eight neighboring cells. Here, to check for contact between the finite elements $\Delta^{(i)}$ and $\Delta^{(j)}$, as shown in Fig. 3.7, first a local coordinate system is defined based on the finite element $\Delta^{(i)}$. Choosing $\mathbf{x}_{1}^{(i)}$ to be the origin, the new coordinate axes are given by the vectors

$$
\mathbf{p}_{2}^{(i)}=\mathbf{x}_{2}^{(i)}-\mathbf{x}_{1}^{(i)}, \quad \mathbf{p}_{3}^{(i)}=\mathbf{x}_{3}^{(i)}-\mathbf{x}_{1}^{(i)}
$$

With respect to the new local coordinate system, the position vectors of the nodes of the finite element $\Delta^{(j)}$ are given by

$$
\mathbf{p}_{1}^{(j)}=\mathbf{x}_{1}^{(j)}-\mathbf{x}_{1}^{(i)}, \quad \mathbf{p}_{2}^{(j)}=\mathbf{x}_{2}^{(j)}-\mathbf{x}_{1}^{(i)}, \quad \mathbf{p}_{3}^{(j)}=\mathbf{x}_{3}^{(j)}-\mathbf{x}_{1}^{(i)}
$$

Next, an edge by edge intersection test is performed. To this end, two interaction parameters are estimated for each node. For example, for the node $\mathbf{p}_{1}^{(j)}$, the interaction parameters are given by

$$
\begin{aligned}
& \lambda_{1}=\frac{\left(\mathbf{p}_{2}^{(i)} \cdot \mathbf{p}_{3}^{(i)}\right)\left(\mathbf{p}_{1}^{(j)} \cdot \mathbf{p}_{3}^{(i)}\right)-\left(\mathbf{p}_{3}^{(i)} \cdot \mathbf{p}_{3}^{(i)}\right)\left(\mathbf{p}_{1}^{(j)} \cdot \mathbf{p}_{2}^{(i)}\right)}{\left(\mathbf{p}_{2}^{(i)} \cdot \mathbf{p}_{3}^{(i)}\right)^{2}-\left(\mathbf{p}_{2}^{(i)} \cdot \mathbf{p}_{2}^{(i)}\right)\left(\mathbf{p}_{3}^{(i)} \cdot \mathbf{p}_{3}^{(i)}\right)} \\
& \lambda_{2}=\frac{\left(\mathbf{p}_{2}^{(i)} \cdot \mathbf{p}_{3}^{(i)}\right)\left(\mathbf{p}_{1}^{(j)} \cdot \mathbf{p}_{2}^{(i)}\right)-\left(\mathbf{p}_{2}^{(i)} \cdot \mathbf{p}_{2}^{(i)}\right)\left(\mathbf{p}_{1}^{(j)} \cdot \mathbf{p}_{3}^{(i)}\right)}{\left(\mathbf{p}_{2}^{(i)} \cdot \mathbf{p}_{3}^{(i)}\right)^{2}-\left(\mathbf{p}_{2}^{(i)} \cdot \mathbf{p}_{2}^{(i)}\right)\left(\mathbf{p}_{3}^{(i)} \cdot \mathbf{p}_{3}^{(i)}\right)} .
\end{aligned}
$$

The finite elements interact if $\lambda_{1}>0, \lambda_{2}>0$ and $\lambda_{1}+\lambda_{2}<1$. The same procedure is followed for the two other nodes of element $\Delta^{(j)}$ and then for all the nodes of element $\Delta^{(i)}$. If the interaction criteria is met for any one of the six cases, the finite elements are assumed to interact and the contact matrix is updated with the contacting pair of finite elements. 


\subsection{Discretized Energy Equation}

The numerical strategies used to estimate each component of the discretized energy equation

are described in this section. At a given time $t_{n}$, for an arbitrary finite element $\Delta^{(j)}$, the element lumped capacitance matrix is given by

$$
\left[\mathcal{M}_{c, n}\right]^{(j)}=\frac{\rho c_{v} A}{3}\left[\begin{array}{ccc}
1 & 0 & 0 \\
0 & 1 & 0 \\
0 & 0 & 1
\end{array}\right]
$$

The final form of the global lumped mass matrix is obtained by summing over all the finite elements as given by Eq. (3.34). Using Eq. (3.8) in Eq. (3.33) and integrating over the element area gives the element stiffness matrix as

$$
\left[\mathcal{K}_{c, n}\right]^{(j)}=\frac{k_{T}}{4 A}\left[\begin{array}{cc}
\beta_{1} & \gamma_{1} \\
\beta_{2} & \gamma_{2} \\
\beta_{3} & \gamma_{3}
\end{array}\right]\left[\begin{array}{lll}
\beta_{1} & \beta_{2} & \beta_{3} \\
\gamma_{1} & \gamma_{2} & \gamma_{3}
\end{array}\right]
$$

where the coefficients in the matrix are given by Eq. (3.9). The final form of the global stiffness matrix is obtained by summing over all the finite elements as given by Eq. (3.34). At time $t_{n}$, the element thermal force matrix is given by

$$
\left[\mathcal{F}_{c, n}\right]_{k}^{(j)}=\int_{\Delta^{(j)}}\left(\dot{Q}_{n}^{e}+\dot{Q}_{n}^{i}\right) N_{k} d A-\int_{\Delta_{c}^{(j)}}\left(q_{f, n}+q_{c, n}\right) N_{k} d l
$$

where $\dot{Q}_{n}^{e}$ and $\dot{Q}_{n}^{i}$ are the thermoelastic and inelastic heating rates evaluated using Eqs. (2.55) and (2.56). For perfect plasticity $(h=0)$, these are estimated as

$$
\dot{Q}_{n}^{e}=-\frac{3 k \alpha \hat{T}_{n}\left(1-\ln \left(J_{n}\right)\right)}{J_{n}^{2}}\left(\mathbf{1}:\left(\mathbf{d}_{n}-\mathbf{d}_{p, n}\right)\right), \quad \dot{Q}_{n}^{i}=\frac{1}{J_{n}} \boldsymbol{\tau}_{n}: \mathbf{d}_{p, n}
$$


respectively, where $\mathbf{d}_{n}$ and $\mathbf{d}_{p, n}$ are estimated as

$$
\mathbf{d}_{n}=\frac{1}{2}\left[\left(\frac{\left(\mathbf{F}_{n}-\mathbf{F}_{n-1}\right) \mathbf{F}_{n}^{-1}}{\Delta t}\right)+\left(\frac{\left(\mathbf{F}_{n}-\mathbf{F}_{n-1}\right) \mathbf{F}_{n}^{-1}}{\Delta t}\right)^{T}\right], \quad \mathbf{d}_{p, n}=\left(\frac{\Delta \epsilon}{\Delta t}\right) \frac{\overline{\boldsymbol{\tau}}_{n}}{\sqrt{\overline{\boldsymbol{\tau}}_{n}: \overline{\boldsymbol{\tau}}_{n}}}
$$

Discretized expressions for the heat flux due to friction and the heat flux required to impose ideal thermal contact conditions are derived next. Their continuous forms are given by Eqs. (2.64) and (2.68), respectively. Since $\mathbf{t}_{t}$ and $\dot{\mathbf{u}}_{t, p}$ are nodal quantities, heat flux on the edge of finite element is estimated by taking the average of the heat flux on its nodes. For example, for the finite element shown in Fig. 3.8(a), if the magnitude of the friction heat flux estimated at node 1 and 2 at time $t_{n}$ are given by $q_{f, n}^{1}$ and $q_{f, n}^{2}$, respectively, then the heat flux associated with edge $1-2 q_{f, n}^{1-2}$ is given by their average.

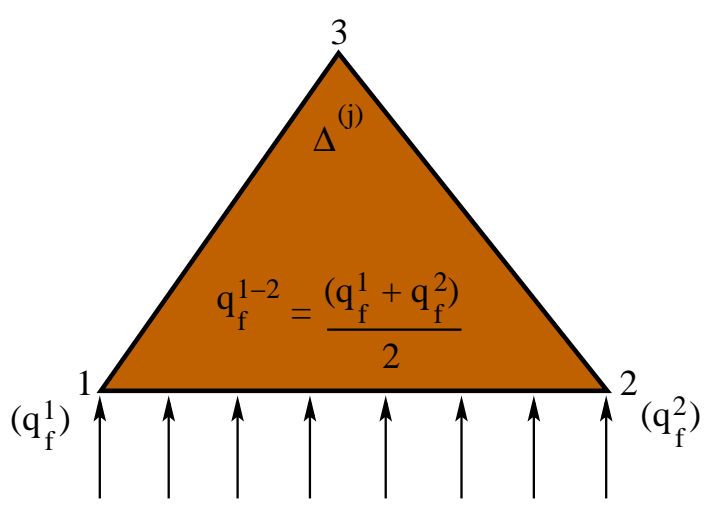

(a)

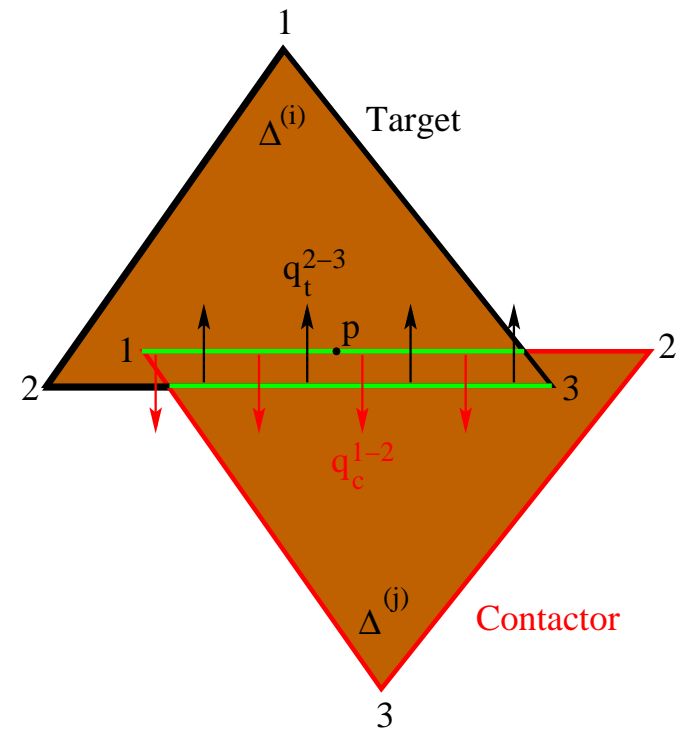

(b)

Figure 3.8: (a) Frictional heat flux. (b) Contact heat flux.

In this study, thermal contact constraints are imposed in the same manner as the kinematic contact constraints, that is, by defining a conservative thermal potential over each finite element and then penalizing the cumulative potential difference between the contacting finite elements. To this end, the thermal potential field at a point $\mathbf{p}$ on the boundary of a contactor finite element 
$\Delta^{(j)}$ that is in contact with a target finite element $\Delta^{(i)}$, as shown in Fig. 3.8(b), is defined as

$$
\Phi_{T}(\mathbf{p})=T_{n}^{(j)}(\mathbf{p})-T_{n}^{(i)}(\mathbf{p})
$$

where $T^{(j)}(\mathbf{p})$ and $T^{(i)}(\mathbf{p})$ are the interpolated temperatures at point $\mathbf{p}$ corresponding to the contactor and target finite elements, respectively. The net potential for $\Delta^{(j)}$ is estimated by summing the potentials at all points $\mathbf{p}$ on the boundary of the contactor element. For example, contribution to the net thermal potential from the contactor edge 1-2 intersecting $\Delta^{(j)}$ is estimated by integrating the individual potentials $\Phi_{T}(\mathbf{p})$ over the edge:

$$
\Phi_{T, c}^{1-2}=\frac{\int_{1}^{2} \Phi_{T}(\mathbf{p}) d l}{l_{1-2}}
$$

where $l_{1-2}$ is the length of the edge 1-2 of $\Delta^{(j)}$. The potential associated with all points $\mathbf{p}$ on the edge 1-2 which do not lie inside $\Delta^{(i)}$ is zero. The corresponding contactor flux associated with the contactor edge 1-2 is then given by

$$
q_{c}^{1-2}=p e^{t h} \Phi_{T, c}^{1-2}
$$

Because the contact heat flux only occurs on the contact boundary of discrete elements, the thermal potential and the contact flux are estimated only for finite element edges on the discretized contact boundary. For all other edges the contact flux is zero. For example, in Fig. 3.8(b), if edge 1-2 of the contactor element belongs to the discretized contact boundary, then $q_{c}^{2-3}=q_{c}^{3-1}=0$. The corresponding contact flux on the target element resulting from the contactor contact flux is given by $-q_{c}^{1-2}$, which is imposed on the edge of the target element that belongs to the discretized contact boundary. For example, if edge 2-3 of the target element is on the discretized contact boundary, then the contact flux on that edge is given by

$$
q_{t}^{2-3}=-q_{c}^{1-2}
$$

The contact flux on edge 1-2 and 3-1 of $\Delta^{(i)}$ is zero. This procedure is followed for all such finite 
element interactions and the resulting contact fluxes on the edges are assembled in a matrix form.

Finally, for any finite element $\Delta^{(j)}$, after integrating over the element area, the first and second term on the right hand side of Eq. (3.89) are given by

$$
\frac{\left(\dot{Q}_{n}^{e}+\dot{Q}_{n}^{i}\right) \Delta}{3}\left[\begin{array}{l}
1 \\
1 \\
1
\end{array}\right]
$$

and

$$
\begin{gathered}
-\frac{\left(q_{f}^{1-2}+q_{c}^{1-2}\right) l_{1-2}}{2}\left[\begin{array}{l}
1 \\
1 \\
0
\end{array}\right] \text { on side 1-2, } \\
-\frac{\left(q_{f}^{2-3}+q_{c}^{2-3}\right) l_{2-3}}{2}\left[\begin{array}{l}
0 \\
1 \\
1
\end{array}\right] \text { on side } 2-3, \\
-\frac{\left(q_{f}^{3-1}+q_{c}^{3-1}\right) l_{3-1}}{2}\left[\begin{array}{l}
1 \\
0 \\
1
\end{array}\right] \text { on side } 3-1,
\end{gathered}
$$

respectively, where $l_{1-2}, l_{2-3}$ and $l_{3-1}$ are the corresponding edge-lengths of $\Delta^{(j)}$. The final form of the global thermal force matrix is obtained by summing over all the finite elements as given by Eq. (3.34). 


\section{Chapter 4}

\section{Verification of the Numerical Method}

The numerical strategies used to model contact between deformable particles, as described in the previous chapter, have been verified against both analytical solutions and computational solutions predicted by the well-established impact mechanics software Ls-Dyna. Results from those studies are presented in this chapter. The discussion is split in two sections. First, studies performed to verify the numerical implementation of the kinematic contact constraints and the constitutive theory are presented in Section 4.1. Second, studies performed to verify the numerical implementation of the thermal contact constraints are presented in Section 4.2. Also presented are convergence studies, which establish the convergence behavior of the numerical method, and sensitivity studies to characterize the dependence of the mechanics and energetics to key numerical parameters such as the normal, tangential and thermal penalty parameters.

\subsection{Verification: Kinematic Contact Constraints}

To verify the numerical implementation of the kinematic contact constraints, the following test problem is considered. Figure 4.1(a) shows two identical right circular cylinders, labeled as $\Omega_{0}^{(a)}$ and $\Omega_{0}^{(b)}$, of radius $R=100 \mu m$ lying with their axes parallel to each other. Both cylinders have identical material properties which are shown in Table 4.1. At time $\mathrm{t}=0, \Omega_{0}^{(a)}$ and $\Omega_{0}^{(b)}$ are given a uniform initial velocity of $\mathbf{v}_{a, 0}=\left[v_{a, 0}^{t}, v_{a, 0}^{n}\right]$ and $\mathbf{v}_{b, 0}=\left[v_{b, 0}^{t}, v_{b, 0}^{n}\right]$, respectively, as shown in the figure.

Three cases are separately considered: elastic, elastic-viscoplastic, and elastic-viscoplasticfrictional impact under plane strain. For all the three cases, the impact period is separated into an initial period of compression and a subsequent period of restitution. During compression, kinetic energy is absorbed by the deformation of the bodies, while during restitution, elastic strain energy generates the force that drives the bodies apart and restores some of the kinetic energy that is absorbed during compression. During elastic-viscoplastic impact some of the initial kinetic 


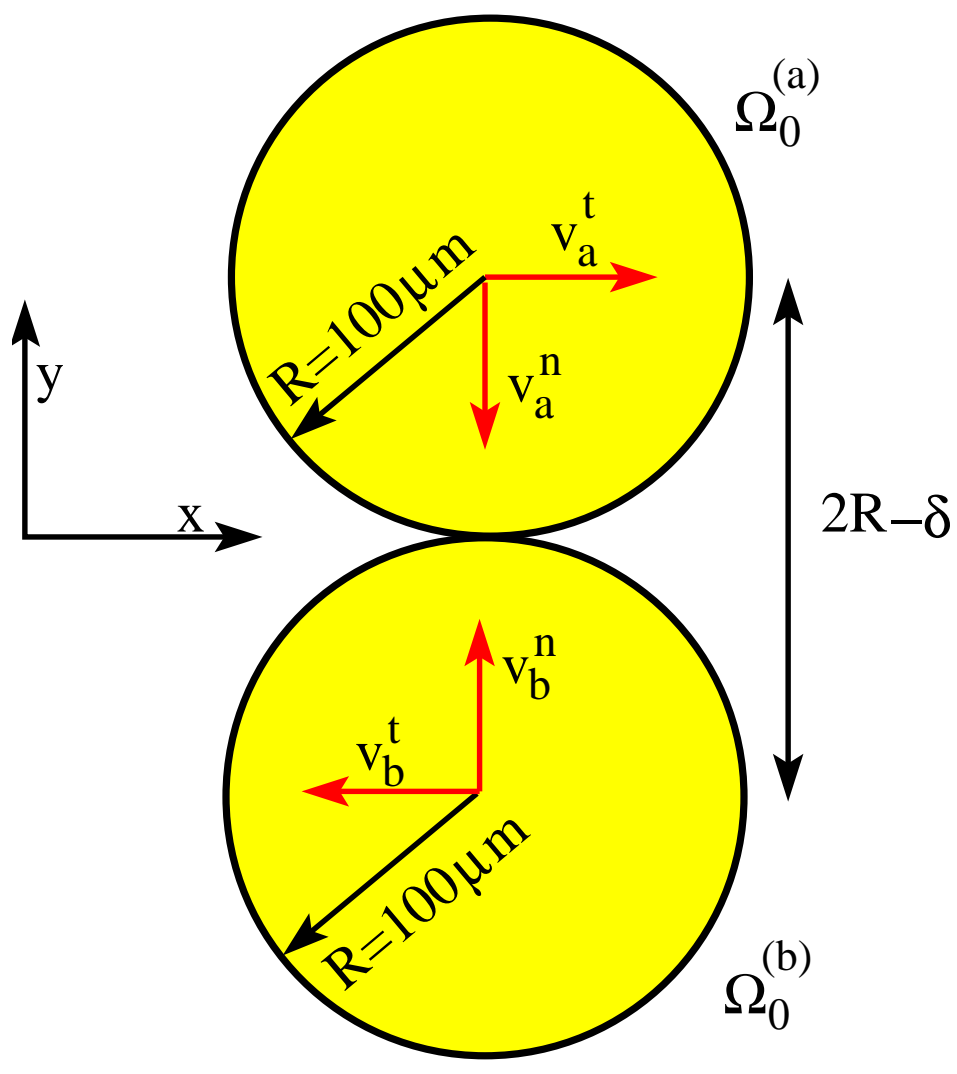

(a)

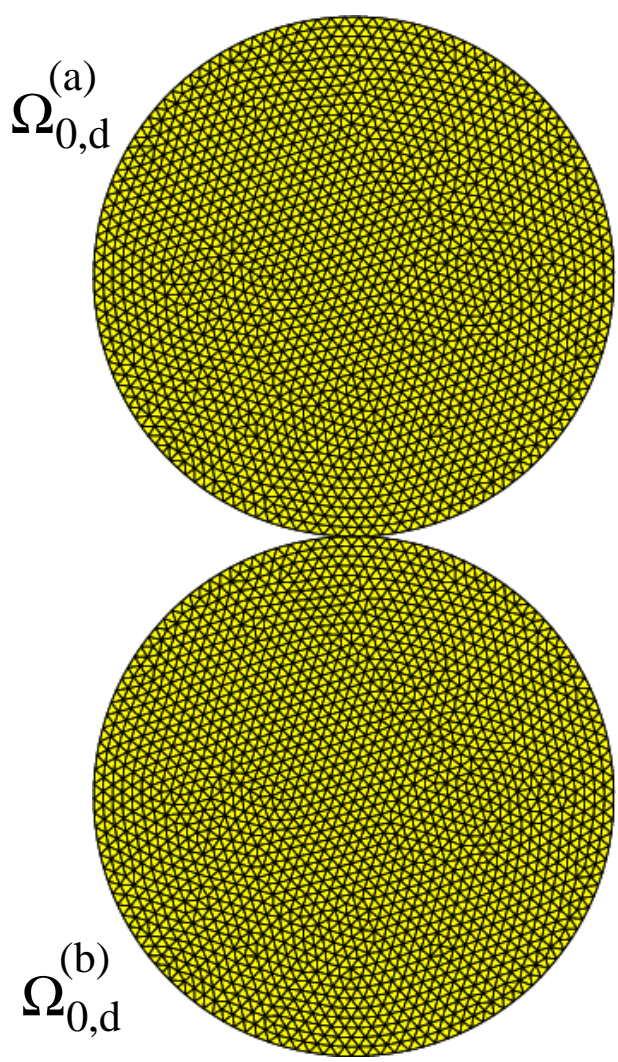

(b)

Figure 4.1: Transverse impact of two identical right circular cylinders in plane strain. (a) Continuous model (b) Discretized model

energy is dissipated due to plasticity. In the elastic-viscoplastic-frictional impact case, energy is dissipated due to plasticity and friction. Numerical solution to all the three test cases is obtained by solving the discretized momentum equation using the combined finite discrete element method on the discretized configuration, as shown in Fig. 4.1(b), where the plane surface of each of the cylinders is discretized into 17803 finite elements comprising of 9060 nodes. Frictionless cases are simulated by setting the friction coefficient to zero. Elastic impact cases are simulated by setting the initial yield strength to a rather large value.

\subsubsection{Elastic Impact}

For purely elastic impact, the numerical results are verified against the analytical solution for non-Hertzian transverse quasi-static contact of two right circular cylinders [63]. Before comparing the numerical and analytical results, the analytical solution is briefly described. When two cylindri- 
Table 4.1: Material properties and numerical parameters used in this study.

\begin{tabular}{lcc}
\hline \hline Parameter & Value & Units \\
\hline \hline Young's Modulus, $E$ & 24.0 & $\mathrm{GPa}$ \\
Poisson's Ratio, $\nu$ & 0.2 & - \\
Initial Density, $\rho_{0}$ & 1903.0 & $\mathrm{~kg} / \mathrm{m}^{3}$ \\
Initial Yield Strength, $\tau_{o}$ & 0.3 & $\mathrm{GPa}$ \\
Friction Coefficient, $\mu$ & 0.25 & - \\
Viscosity, $\gamma$ & 100 & $\mathrm{~Pa} . \mathrm{s}$ \\
Hardening Modulus, $h$ & 0.0 & - \\
Thermal Conductivity, $k_{T}$ & 0.5 & $\mathrm{~W} / \mathrm{m}-\mathrm{K}$ \\
Specific Heat, $c_{v}$ & 1500.0 & $\mathrm{~J} / \mathrm{kg}-\mathrm{K}$ \\
Thermal Expansion Coefficient, $\alpha$ & $1.0 \times 10^{-5}$ & $/ \mathrm{K}$ \\
Penalty Parameter Normal, $p e^{n}$ & $24 \times 10^{9}$ & - \\
Penalty Parameter Tangential, $p e^{t}$ & $1 \times 10^{11}$ & - \\
Penalty Parameter Thermal, $p e^{t h}$ & $1 \times 10^{11}$ & - \\
Time Increment, $\Delta t$ & 0.01 & $\mathrm{~ns}$ \\
\hline \hline
\end{tabular}

cal bodies with their axes parallel are pressed together in contact by a force $p$ per unit length, they make contact over a long strip of width $2 a$ parallel to their axis. Under the effect of the compressive load the bodies approach each other by a distance $\delta$. Assuming that both the contact surfaces are continuous, non-conforming, and frictionless, and that the strains are small compared to the size of the bodies, the Hertzian solution relates radial compression $\delta$ to the compressive load $p$ as

$$
\delta=\frac{2 p}{\pi E^{*}}\left(\log \left(\frac{4 \pi R E^{*}}{p}\right)-1\right)
$$

where $E^{*}=E /\left(1-\nu^{2}\right)$. Here, $E$ is the Young's modulus and $\nu$ is the Poisson's ratio. During contact, the compressive force $p$ acts on each cylinder to change its velocity. At any instant in time, if $\mathbf{v}_{a}=\left[v_{a}^{t}, v_{a}^{n}\right]$ and $\mathbf{v}_{b}=\left[v_{b}^{t}, v_{b}^{n}\right]$ are the velocities of the center of mass of the particles $\Omega^{(a)}$ and $\Omega^{(b)}$, respectively, then for purely normal impact, that is, $v_{a, 0}^{t}=v_{b, 0}^{t}=0$, the equations of motion reduce to

$$
m \frac{d v_{a}^{n}}{d t}=p, \quad m \frac{d v_{b}^{n}}{d t}=-p
$$


where $m$ is the mass per unit length of $\Omega^{(a)}$ and $\Omega^{(b)}$. Also, during contact

$$
\frac{d \delta}{d t}=v_{b}^{n}-v_{a}^{n}
$$

Using Eq. (4.2) in Eq. (4.3) gives

$$
\frac{d^{2} \delta}{d t^{2}}=\frac{-2 p}{m}
$$

The initial conditions for the above ODE are given as: at $\mathrm{t}=0 ; \delta=0, p=0$ and $d \delta / d t=v_{0}^{n}$, where $v_{0}^{n}=v_{b, 0}^{n}-v_{a, 0}^{n}$ is the initial relative normal velocity. For a given $v_{0}^{n}$, the analytical solution is obtained by solving the ODE simultaneously with Eq. (4.1) for $\delta$ and $p$. Here, it is assumed that the deformation is quasi-static, the elastic wave motion has no effect on the solution, and that at any instant each cylinder is moving with the velocity of its center of mass. For verification purposes, numerous sets of numerical and analytical results are obtained by varying $v_{0}^{n}$. The cases presented here are shown in Table 4.2. Predictions from these cases are discussed next.

Table 4.2: Cases presented for verification of elastic, elastic-viscoplastic and elastic-viscoplastic-

\begin{tabular}{|c|c|c|c|c|c|c|c|c|}
\hline \multicolumn{3}{|c|}{ Elastic } & \multicolumn{3}{|c|}{ Elastic-Viscoplastic } & \multicolumn{3}{|c|}{ Elastic-Viscoplastic-Frictional } \\
\hline Case & $v_{0}^{n}$ & $v_{0}^{t}$ & Case & $v_{0}^{n}$ & $v_{0}^{t}$ & Case & $v_{0}^{n}$ & $v_{0}^{t}$ \\
\hline $\mathbf{E}_{1}$ & $5 \mathrm{~m} / \mathrm{s}$ & $0 \mathrm{~m} / \mathrm{s}$ & $\mathbf{P}_{1}$ & $50 \mathrm{~m} / \mathrm{s}$ & $0 \mathrm{~m} / \mathrm{s}$ & $\mathbf{F}_{1}$ & $50 \mathrm{~m} / \mathrm{s}$ & $120 \mathrm{~m} / \mathrm{s}$ \\
\hline $\mathbf{E}_{2}$ & $50 \mathrm{~m} / \mathrm{s}$ & $0 \mathrm{~m} / \mathrm{s}$ & $\mathbf{P}_{2}$ & $100 \mathrm{~m} / \mathrm{s}$ & $0 \mathrm{~m} / \mathrm{s}$ & $\mathbf{F}_{2}$ & $300 \mathrm{~m} / \mathrm{s}$ & $360 \mathrm{~m} / \mathrm{s}$ \\
\hline $\mathbf{E}_{3}$ & $100 \mathrm{~m} / \mathrm{s}$ & $0 \mathrm{~m} / \mathrm{s}$ & $\mathbf{P}_{3}$ & $200 \mathrm{~m} / \mathrm{s}$ & $0 \mathrm{~m} / \mathrm{s}$ & - & - & - \\
\hline $\mathbf{E}_{4}$ & $300 \mathrm{~m} / \mathrm{s}$ & $0 \mathrm{~m} / \mathrm{s}$ & $\mathbf{P}_{4}$ & $300 \mathrm{~m} / \mathrm{s}$ & $0 \mathrm{~m} / \mathrm{s}$ & - & - & - \\
\hline
\end{tabular}
frictional impact.

Shown in Fig. 4.2 are the energy history profiles of the particles through the impact duration for all the cases. All the quantities are plotted as a percentage of the total initial energy of the particles, estimated as, $E_{0}=0.5 m\left(v_{0}^{n}\right)^{2}$. Each plot shows the variation in the total kinetic energy $E_{k}$ and total deformation work $W_{d}$ during impact, obtained by numerically integrating Eqs. (2.52) and (2.48), respectively. Here, $W_{d}$ consists of total elastic shear strain energy $W_{s}$ and compression work $W_{c}$. Also plotted is the the total energy of the two bodies, $E_{T}$, estimated as $E_{T}(t)=E_{k}(t)+W_{d}(t)$. For each case, impact results in reversible conversions between $E_{k}$ and 
$W_{d}$. Although $E_{k}$ and $W_{d}$ change during impact, their sum must remain constant and equal to $E_{0}$. The percentage numerical error $\epsilon$, which is estimated as $\epsilon=100 *\left|E_{0}-E_{T}\left(t_{f}\right)\right| / E_{0}$, for all the simulations is less than $1.0 \times 10^{-2} \%$. This error is due to the small amount of numerical dissipation inherent in the method.

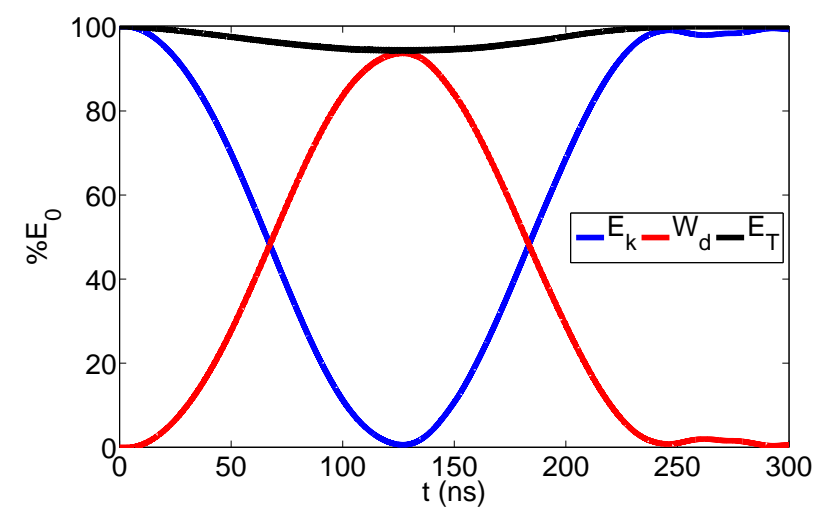

(a) Case $\mathbf{E}_{1}$

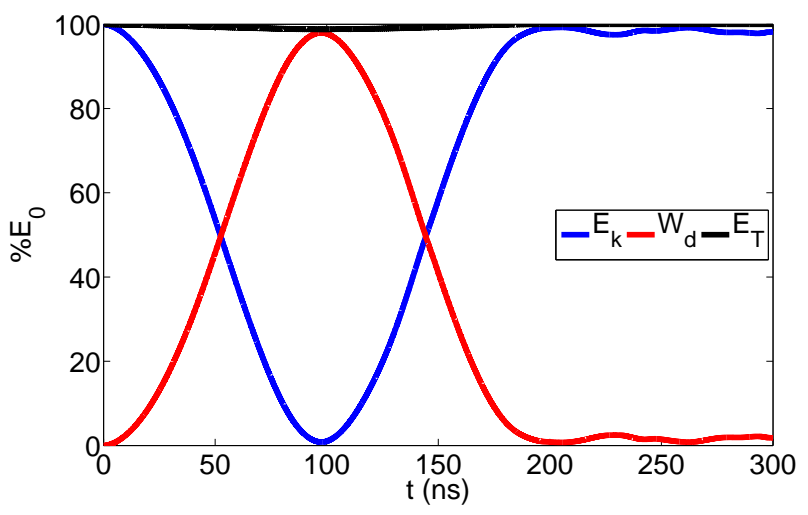

(c) Case $\mathbf{E}_{3}$

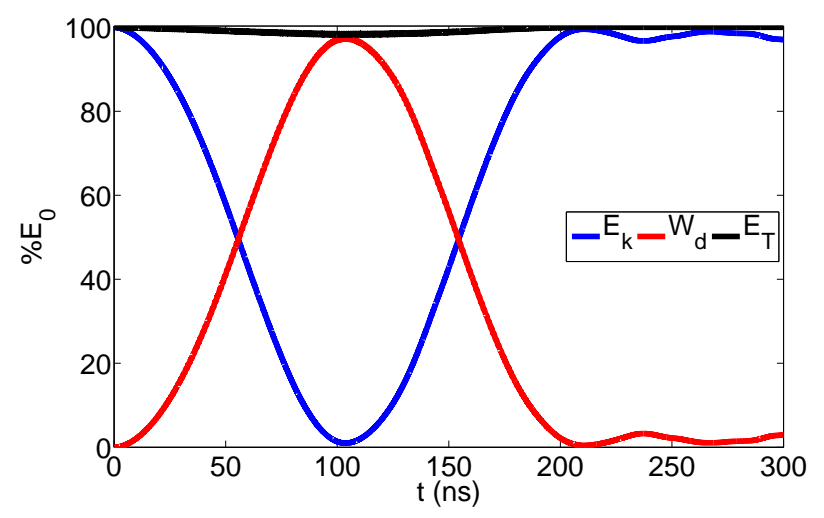

(b) Case $\mathbf{E}_{2}$

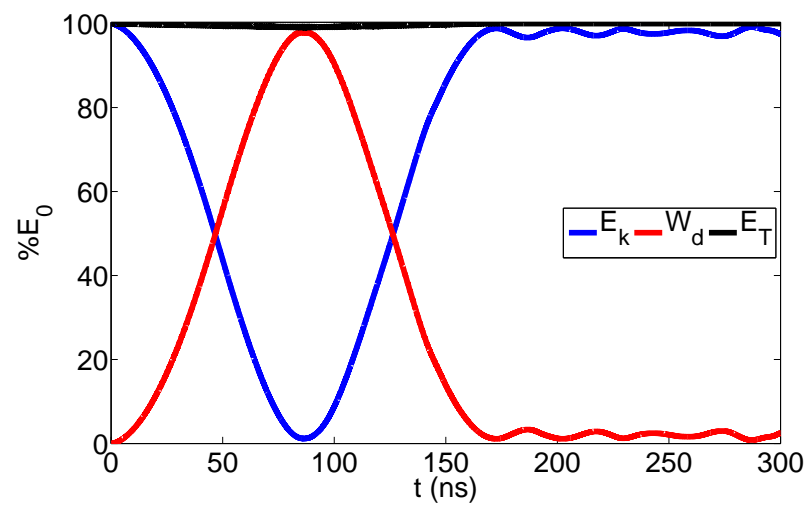

(d) Case $\mathbf{E}_{4}$

Figure 4.2: Energy history profiles for elastic impact of right circular cylinders.

A few other observations are noteworthy. First, the total contact duration time $t_{f}$ decreases with $v_{0}^{n}\left(t_{f} \approx 250,210,197\right.$ and 170 ns for Case $\mathbf{E}_{1}, \mathbf{E}_{2}, \mathbf{E}_{3}$ and $\mathbf{E}_{4}$, respectively). Second, the slight decrease in total energy that is predicted during impact is a consequence of the artificial penetration energy associated with penalty based contact methods. In this method, the conservative gap potential acts as a reservoir, which stores energy in the form of recoverable penetration energy during the compression phase and feeds it back to the body during the release phase analogous to an elastic spring. This artificial energy, which is estimated as $E_{\phi}(t)=E_{0}-E_{T}(t)$, directly depends on the amount of penetration between the two contacting surfaces through the normal penalty 
parameter $p e^{n}$ and the size of the finite elements at the contact interface. Penetration decreases with the size of the finite elements and is minimum when $p e^{n} \rightarrow \infty$, which implies $E_{\phi}(t) \rightarrow 0$. However, large penalty terms result in numerical stiffness problems that can cause a simulation to crash. Whereas, small penalty terms result in high residual error resulting from an inaccurate description of the strain field. Therefore, choosing the right penalty parameter is essential to obtaining an accurate and physical solution. The sensitivity of the predictions to the normal penalty parameter will be discussed later. For the verification studies, the penalty parameter is chosen to be equal to the Young's modulus of the particles. Third, impact, in the absence of dissipation, induces higher amplitude elastic vibrations (high frequency elastic ringing) within the particles. These are indicated by the oscillations in $E_{k}$ and $W_{d}$ profiles after impact. These oscillations are indicative of alternating compression and expansion of the particles resulting from stress wave reflections within them. The amplitude and frequency of these oscillations increase with $v_{0}^{n}$. Therefore, even for purely elastic impact, not all of the initial kinetic energy is recovered after impact.

The numerical and analytical predictions for the evolution of $\delta$ and $p$ for Cases $\mathbf{E}_{1}, \mathbf{E}_{2}$ and $\mathbf{E}_{3}$ are shown in Fig. 4.3. The numerical estimate for radial compression $\delta_{n}$ is obtained by tracking the displacement of the center of mass of the particles through the impact duration time, whereas the numerical compressive load $p_{n}$ for each cylinder is obtained by summing up all the normal nodal forces on the contact surface. Profiles of $p_{n}$ show high frequency oscillations whose amplitude increases with $v_{0}^{n}$. This is an inherent characteristic of the penalty method. As mentioned, this method penalizes violations in the gap constraints by multiplying them with a rather large parameter $p e^{n}$. Because $p e^{n}$ is predetermined and the gap potential varies during impact, contact constraints are not exactly enforced at each time step. Subsequently, the penalty method leads to unwanted and non-physical oscillations in contact forces. However, the amplitude of these oscillations can be minimized by choosing the right penalty parameter. As shown later, for the value of $p e^{n}$ used in this study, these oscillations have minimal effect on the solution. The summarized numerical and analytical predictions for the elastic case are presented in Fig. 4.4. These figures 
Case $\mathbf{E}_{1}$
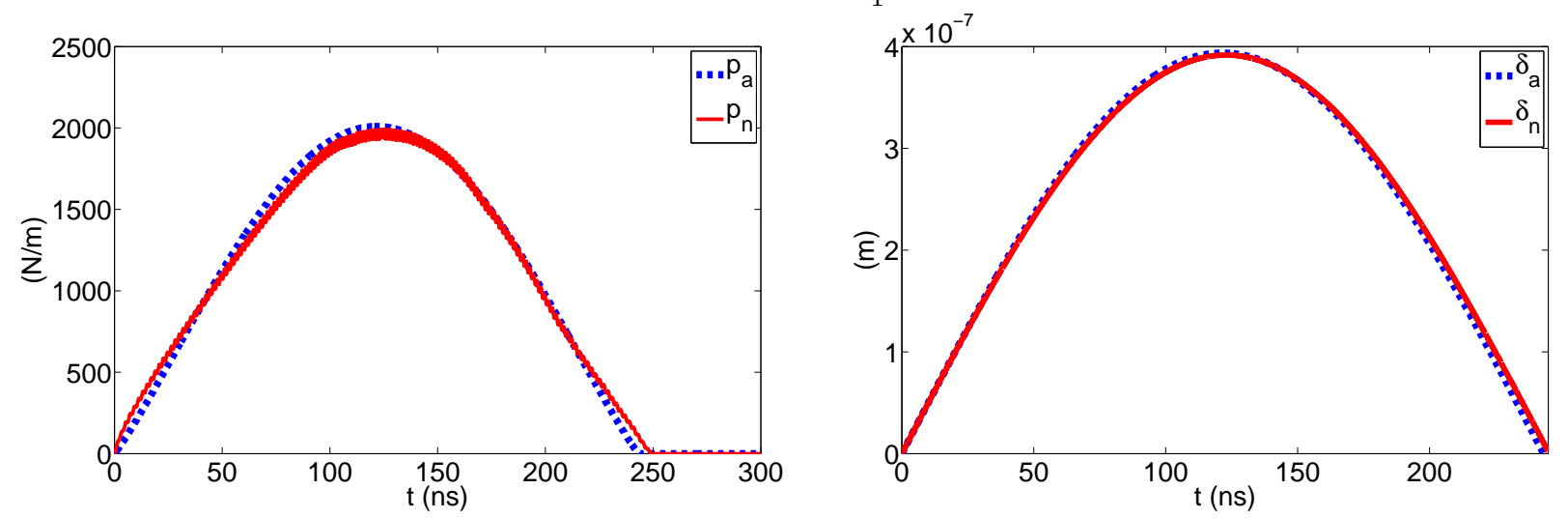

Case $\mathbf{E}_{2}$
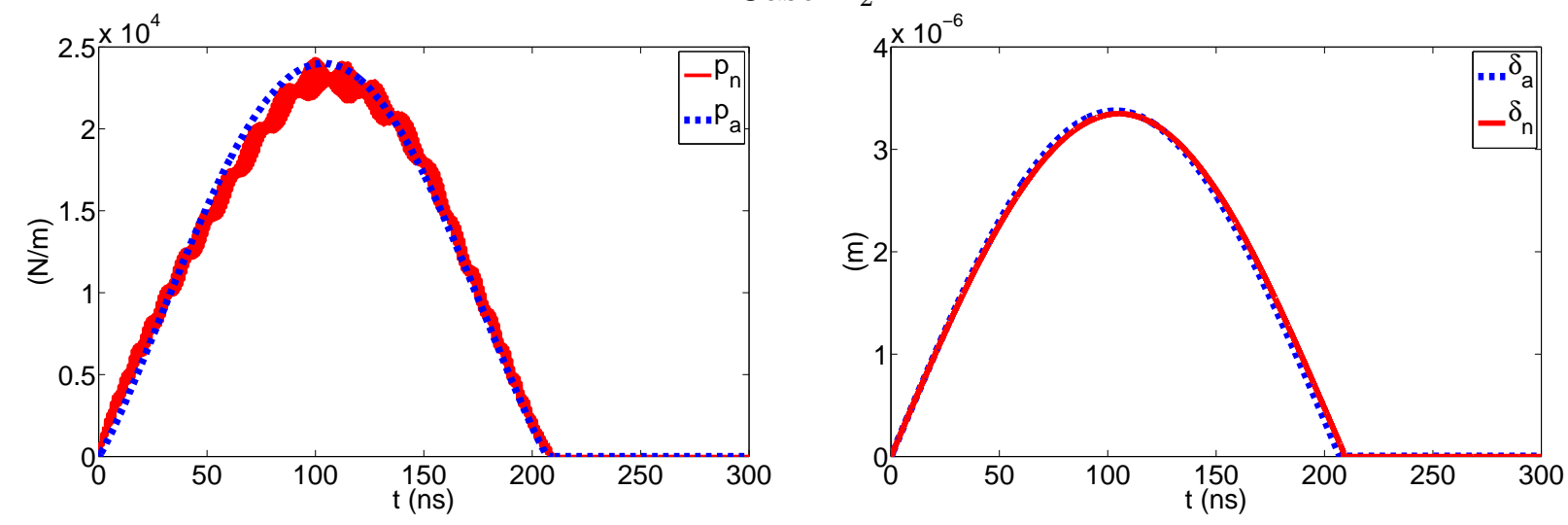

Case $\mathbf{E}_{3}$

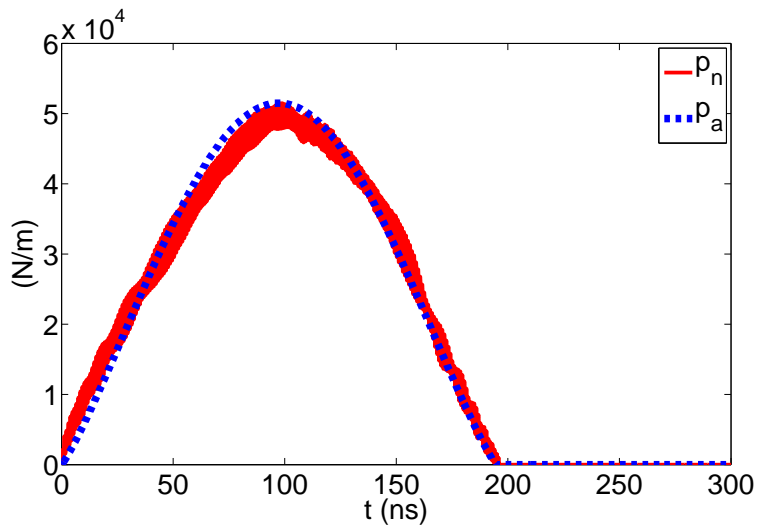

(a) Compressive Load

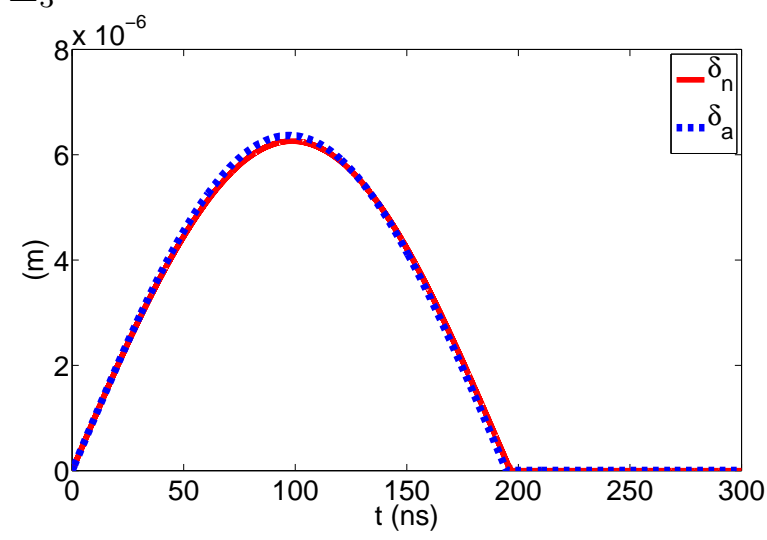

(b) Radial Compression

Figure 4.3: History profiles of (a) Compressive Load and (b) Radial Compression for elastic impact of right circular cylinders.

show the variation in the maximum radial compression $\delta^{\text {max }}$ and maximum compressive load $p^{\max }$ with respect to $v_{0}^{n}$. From the figures, it is evident that the numerical predictions agree very well with the analytical results. 


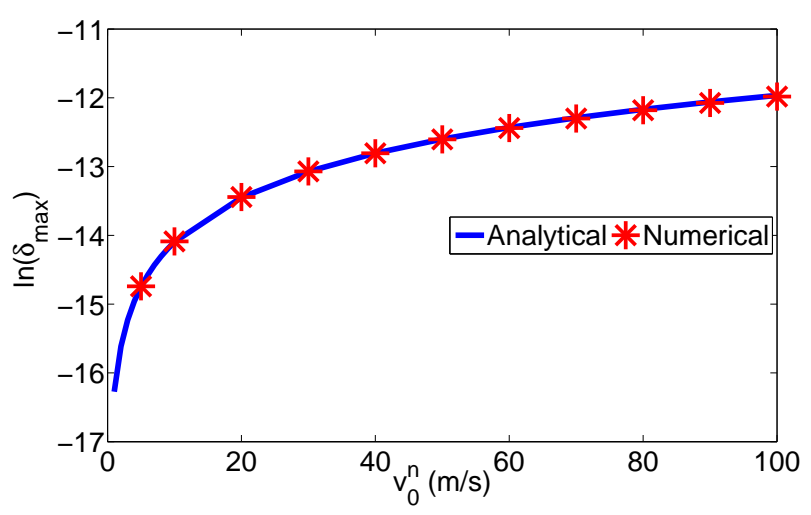

(a)

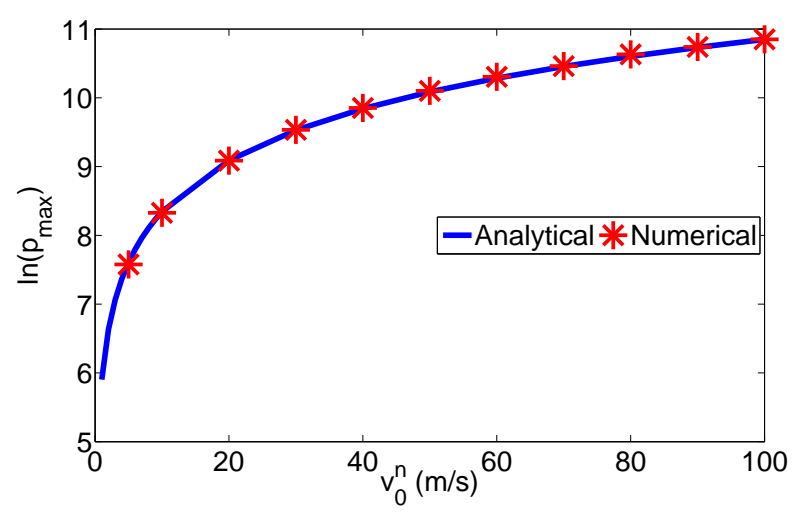

(b)

Figure 4.4: Variation of (a) maximum radial compression and (b) maximum compressive load with $v_{0}^{n}$.

\subsubsection{Elastic-Viscoplastic Impact}

Due to lack of analytical results, for verification purposes, the numerical predictions for the elastic-viscoplastic test cases are compared to the numerical solutions obtained from the well established commercially available impact mechanics software Ls-Dyna. The test case is recreated using Ls-Dyna with the same discretized initial configuration and material model. For comparison purposes, numerous sets of numerical predictions are obtained using the FDEM (combined finite discrete element method) and Ls-Dyna by varying the initial relative normal velocity. The cases presented in this study are shown in Table 4.2. Predictions from these cases are discussed next.

First, to illustrate the impact process, contours of the total plastic work $W_{p}$ within the two particles are shown in Fig. 4.5. Here, $v_{0}^{n}=500 \mathrm{~m} / \mathrm{s}$. During impact, plastic yield is initiated underneath the contact surface when the maximum effective stress exceeds the initial yield strength. As impact proceeds further, the plastic zone expands towards the contact surface but still being encompassed by the surrounding material that is elastically deformed. After approximately $20 \mathrm{~ns}$, plastic zone reaches the surface after which it expands until the entire contact region consists of the plastic zone. Plastic work, which is concentrated below the contact interface during the initial phase $(t<40 \mathrm{~ns})$, is concentrated near the periphery during the later stages.

Shown in Fig. 4.6 are the energy history profiles of the particles for all the cases. All the quantities are plotted as a percentage of the total initial energy of the particles $E_{0}$. Each plot 

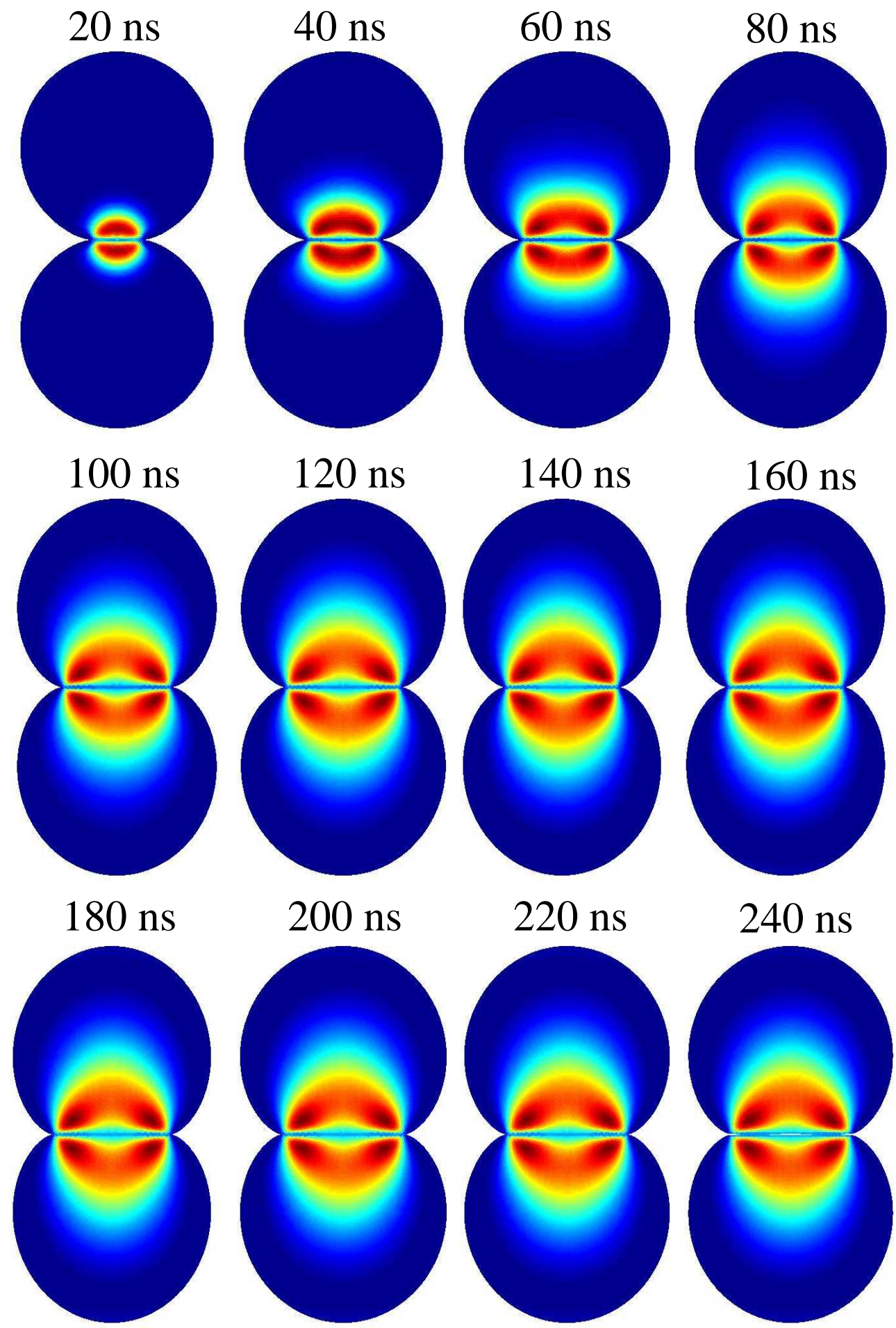

Figure 4.5: Spatial and temporal evolution of plastic work per unit mass resulting from elasticviscoplastic impact at $v_{0}^{n}=500 \mathrm{~m} / \mathrm{s}$. 
shows the variation in $E_{k}, W_{d}$ and $E_{T}$ during impact. Also plotted are the predictions for the total kinetic energy $E_{k}^{*}$ and deformation work $W_{d}^{*}$ obtained from Ls-Dyna. For these cases, $W_{d}$ represents the sum of the total elastic strain energy $W_{s}$, compression work $W_{c}$ and plastic work $W_{p}$. Like the elastic cases, elastic-viscoplastic impact also results in conversions between $E_{k}$ and $W_{d}$. However, for these cases, a large portion of the initial kinetic energy is dissipated due to plastic work and only a small portion is recovered after impact. Approximately, $50 \%$ of $E_{0}$ is dissipated for Case $\mathbf{P}_{1}$, which increases to $95 \%$ for Case $\mathbf{P}_{4}$. Also, dissipation eliminates high frequency elastic ringing within the particles. Figure 4.7(a) summarizes the predicted variation in coefficient of restitution $(e)$, which is estimated as $e=\left|E_{k}(t=0)-E_{k}\left(t=t_{f}\right)\right| / E_{k}(t=0)$, with $v_{0}^{n}$. Also shown are corresponding values predicted by Ls-Dyna. Both sets of predictions indicate that $e$ is close to unity for small impact velocities but decreases with increasing $v_{0}^{n}$. The predictions of the FDEM and those of Ls-Dyna agree well. The percentage numerical error $\epsilon$, which is estimated as $\epsilon=100 *\left|E_{0}-E_{T}\left(t_{f}\right)\right| / E_{0}$, for all the simulations is less than $1.0 \times 10^{-2} \%$.

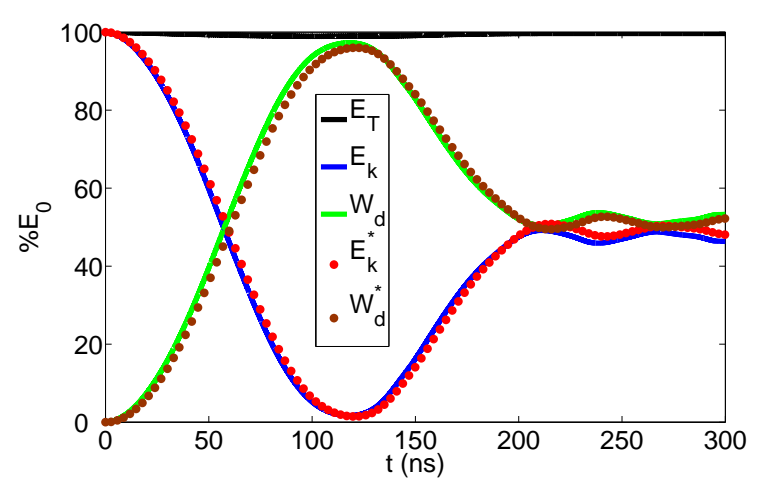

(a) Case $\mathbf{P}_{1}$

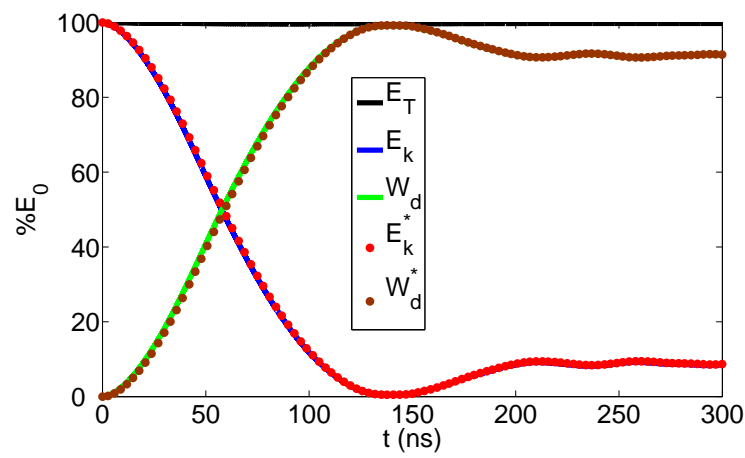

(c) Case $\mathbf{P}_{3}$

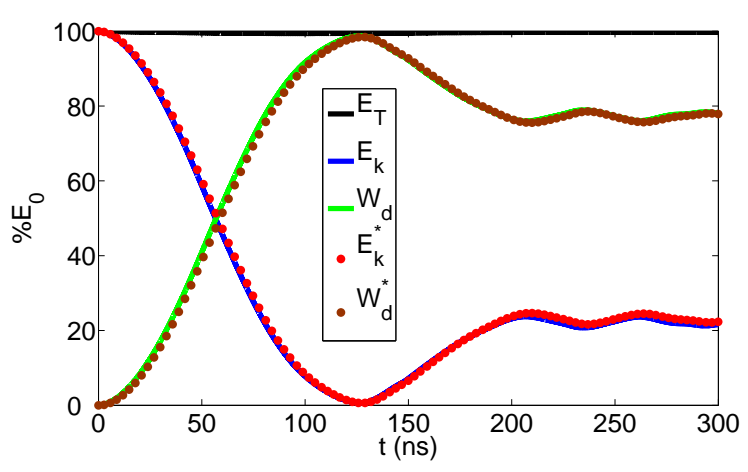

(b) Case $\mathbf{P}_{2}$

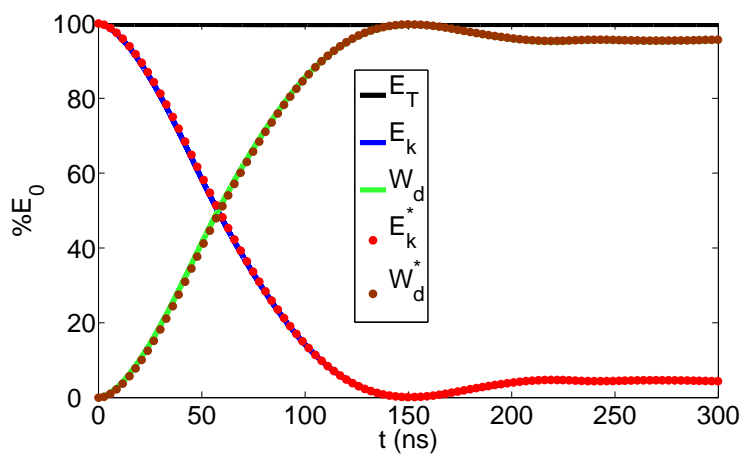

(d) Case $\mathbf{P}_{4}$

Figure 4.6: Energy history profiles for elastic-viscoplastic impact of right circular cylinders. 


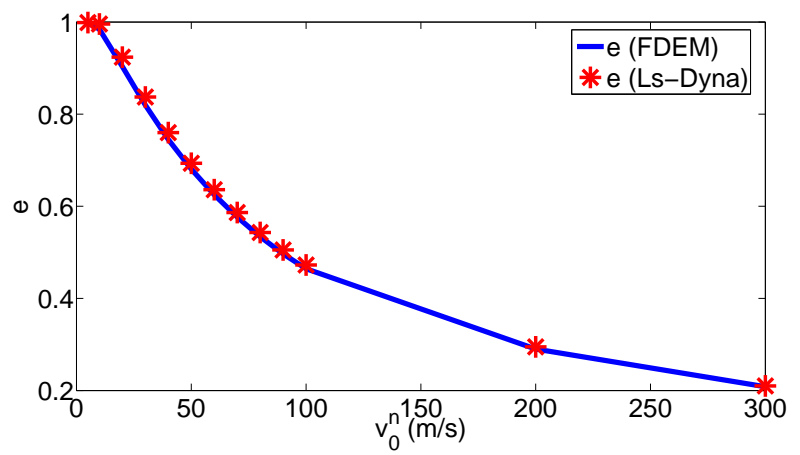

Figure 4.7: (a) Variation in coefficient of restitution with $v_{0}^{n}$ for elastic-viscoplastic impact

Figure 4.8 show contours of pressure and in-plane shear stress within the two particles at $t=$ 150 ns for Case $\mathbf{P}_{4}$. Figures shown on the left are Ls-Dyna predictions whereas figures on the right are predictions from the FDEM. Values in the color-bars are in GPa. It is evident in the figures that impact results in complex stress fields within the particles. Here, an exceptionally fine mesh is used to capture the spatial variation of the stress fields. In practice, it is not computationally feasible to use this level of discretization to solve the particle ensemble problem. The figures indicate that normal impact induces symmetric pressure fields and anti-symmetric shear stress fields. Also, stress concentrations occur near the periphery of the contact interface resulting in localized plastic work as shown in Fig. 4.5. The pressure and shear stress fields predicted by the FDEM and Ls-Dyna agree well.

\subsubsection{Elastic-Viscoplastic-Frictional Impact}

Comparisons are presented in this section between numerical predictions from the FDEM and Ls-Dyna for elastic-viscoplastic-frictional impact of two identical right circular cylinders in plane strain. For comparison purposes, numerous sets of results are obtained using the FDEM and LsDyna by varying the initial normal $\left(v_{0}^{n}\right)$ and tangential $\left(v_{0}^{t}\right)$ relative velocity. The cases presented in this study are shown in Table 4.2. Predictions from these cases are discussed next.

Figure 4.9 shows the energy history profiles of the particles for both the cases. All the quantities are plotted as a percentage of the total initial energy of the particles $E_{0}$, which is evaluated as $E_{0}=0.5 m\left(\left(v_{0}^{n}\right)^{2}+\left(v_{0}^{t}\right)^{2}\right)$. Each plot shows the variation in the total kinetic energy $E_{k}$, 


\section{Pressure}

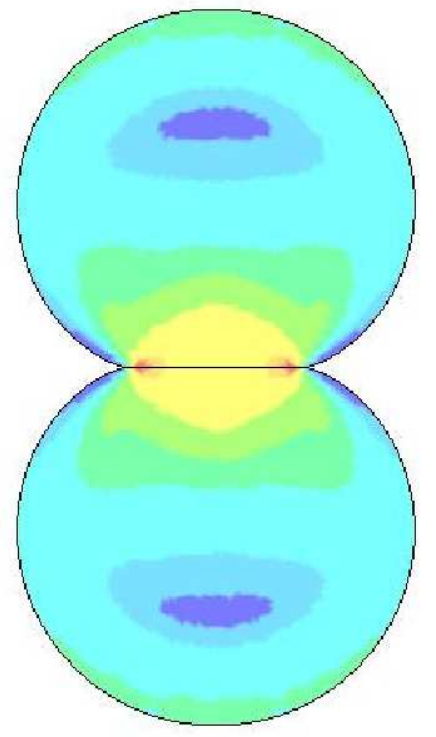

Fringe Levels

4.934e-01

4.280e-01

$3.626 e-01$

2.972e-01

$2.317 e-01$

$1.663 \mathrm{e}-01$

$1.009 \mathrm{e}-01$

$3.553 e-02$

$-2.988 \mathrm{e}-02$

$-9.529 \mathrm{e}-02$

$-1.607 \mathrm{e}-01$

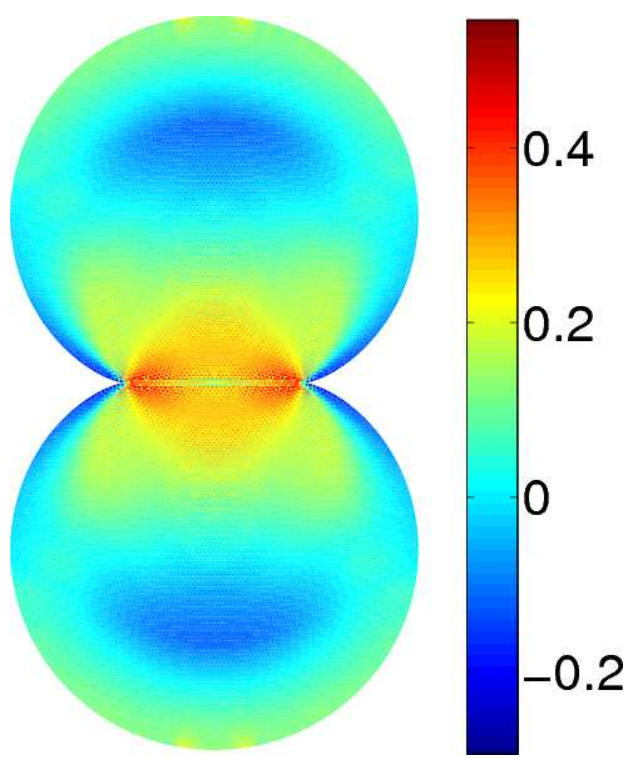

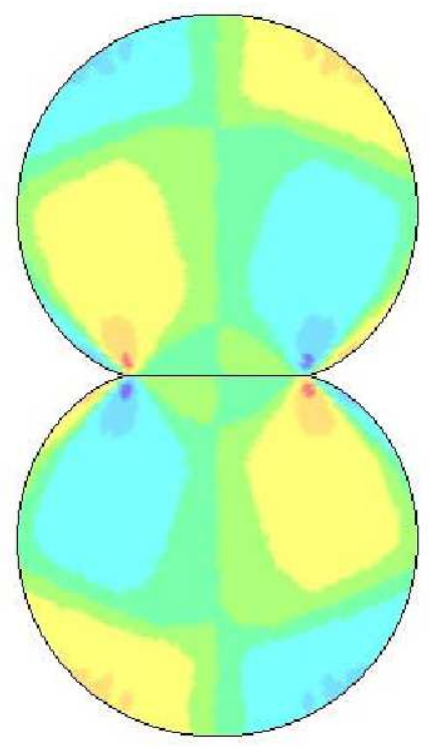

(a)
In-Plane Shear Stress
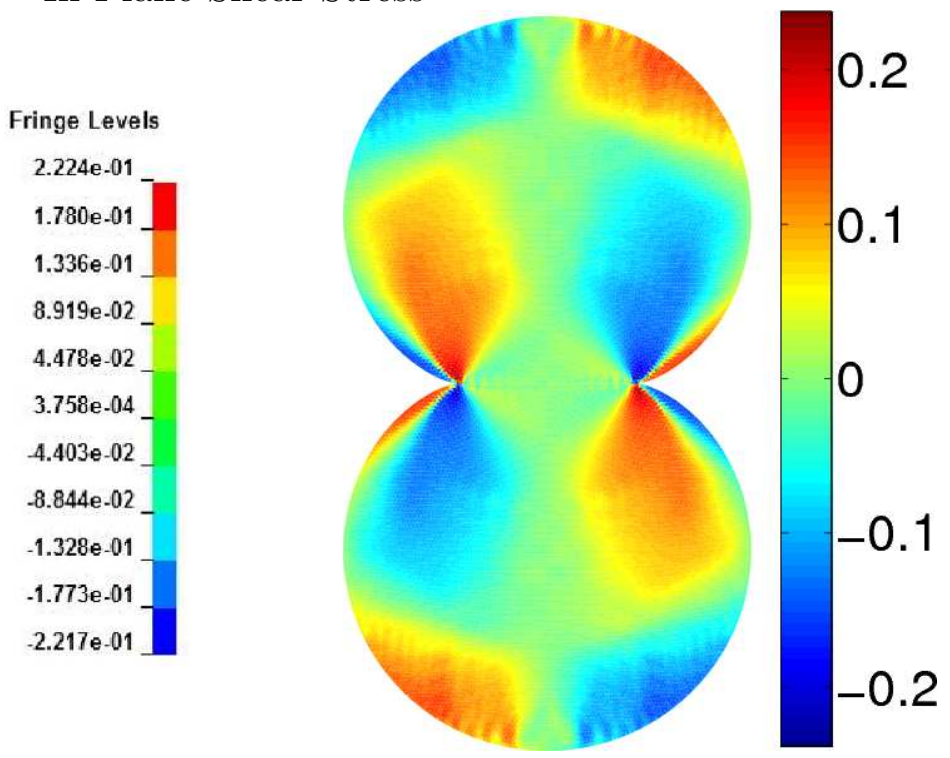

(b)

Figure 4.8: Spatial contours of pressure and in-plane shear stress for Case $\mathbf{P}_{4}$ (a) Ls-Dyna and (b) FDEM. All values shown in the color-bar are in GPa. 


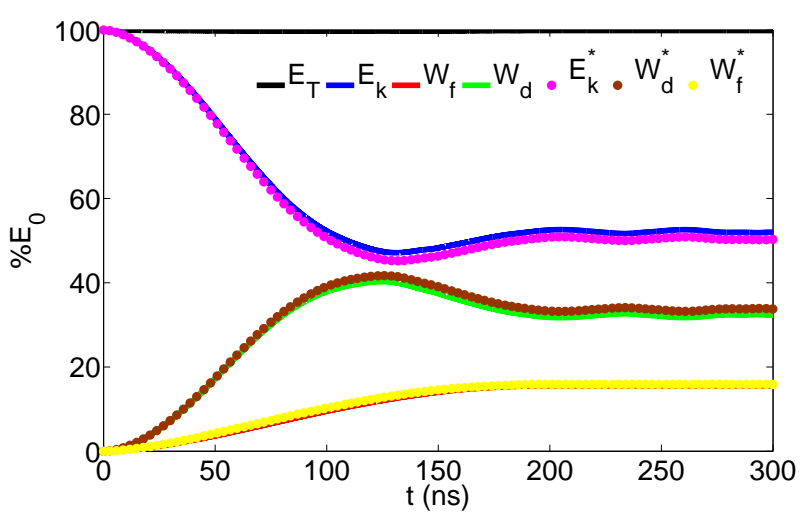

(a) Case $\mathbf{F}_{1}$

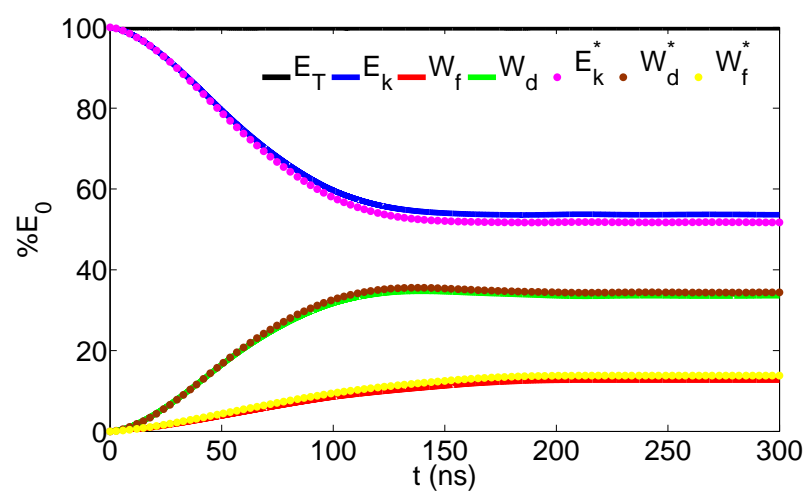

(b) $\mathbf{F}_{2}$

Figure 4.9: Energy history profiles for elastic-viscoplastic-frictional impact of right circular cylinders.

the total deformation work $W_{d}$, and the total friction work $W_{f}$, which is estimated by numerically integrating Eq. (2.53) during impact. For these cases, the total energy $E_{T}$ is estimated as $E_{T}(t)=E_{k}(t)+W_{d}(t)+W_{f}(t)$. Also plotted are the Ls-Dyna predictions for the total kinetic energy $E_{k}^{*}$, deformation work $W_{d}^{*}$ and friction work $W_{f}^{*}$. During contact, $W_{f}$ increases steadily from time $t=0$ till the particles separate. It constitutes $16 \%$ and $13 \%$ of the initial energy for Cases $\mathbf{F}_{1}$ and $\mathbf{F}_{2}$, respectively. Plastic work constitutes $33 \%$ and $36 \%$ of the initial energy for Cases $\mathbf{F}_{1}$ and $\mathbf{F}_{2}$, respectively. The net plastic work for the Cases $\mathbf{P}_{2}$ and $\mathbf{F}_{1}$, whose initial normal velocity is the same $\left(v_{0}^{n}=100 \mathrm{~m} / \mathrm{s}\right)$, is 0.1052 and $0.1073 \mathrm{~J} / \mathrm{m}$. This indicates that plastic work in minimally influenced by friction. The numerical predictions from the FDEM agree very well with Ls-Dyna results. The percentage numerical error $\epsilon$, which is estimated as $\epsilon=100 *\left|E_{0}-E_{T}\left(t_{f}\right)\right| / E_{0}$, for all the simulations is less than $1.0 \times 10^{-2} \%$.

Figure 4.10 shows spatial contours of shear stress within the particles for Cases $\mathbf{F}_{1}$ and $\mathbf{F}_{2}$ at $t=100 \mathrm{n} / \mathrm{s}$. Figures shown on the left are Ls-Dyna predictions whereas figures on the right are predictions from the FDEM. Values shown in the color-bars are in GPa. The figures indicate that oblique impact induces spatially biased fields. During impact, complex stick-slip frictional dynamics at the contact interface induce local stress concentrations on the periphery of the contact interface resulting in localized plastic work. It is also generally accepted that friction work is most significant in regions where the surface shear stress is large [1]. These predictions are consistent 
with experiments which indicate that hot-spots often occur on particle contact surfaces [26]. The results from the FDEM match very well with Ls-Dyna results.

Figure 4.11 shows the summarized plots of the variation in the frictional dissipation $W_{f}$ with respect to the impact incidence angle $\phi^{\circ}$ and the friction coefficient $\mu$. In this analysis, the impact angle is changed by changing the initial normal and tangential velocity while keeping the net initial relative speed constant at $300 \mathrm{~m} / \mathrm{s}$. For example, impact at an angle $\phi^{\circ}$ is simulated by setting $v_{a, 0}^{n}=v_{b, 0}^{n}=150 * \cos \left(\phi^{\circ}\right) \mathrm{m} / \mathrm{s}$ and $v_{a, 0}^{t}=v_{b, 0}^{t}=150 * \sin \left(\phi^{\circ}\right) \mathrm{m} / \mathrm{s}$. Sensitivity of the friction work to $\mu$ is demonstrated by fixing $\phi^{\circ}=45^{\circ}$ and varying $\mu$. Also plotted are the corresponding values obtained from Ls-Dyna. Detailed examination of the variation in frictional mechanics with $\phi^{\circ}$ indicates a complex response involving stick, slip-stick reversal, and gross slip. For approximately $0^{\circ} \leq \phi^{\circ} \leq 20^{\circ}$, much of the contact surface sticks due to locally high normal stresses, though some micro-slip occurs near the surface edge, where normal stresses become small, resulting in small frictional dissipation. For approximately $20^{\circ}<\phi^{\circ} \leq \phi_{c}^{\circ}=70^{\circ}$, the contact surface initially slips, followed by a brief period of stick, before slipping again in the reverse direction, collectively resulting in an increase in dissipation. Here, slip is not restricted to the surface edge, but largely occurs over the entire surface. Gross slip is predicted to occur for $\phi^{\circ}>\phi_{c}^{\circ}$, where the decrease in dissipation results from a significant reduction in normal contact stress. Figure 4.11(a) indicates that the FDEM results match very well with Ls-Dyna results for $\phi^{\circ}<65^{\circ}$ beyond which the FDEM under-predicts the frictional dissipation.

The results of Fig. 4.11(b) indicate that there exists a particular value of friction coefficient, for fixed value of initial relative speed and impact angle, that maximizes frictionally dissipated energy. A maximum value of $16.16 \%$ occurs near the critical value $\mu_{c}=0.3$. A sharp increase in the percentage of frictionally dissipated energy is predicted for $\mu<\mu_{c}$, whereas a more gradual decrease is predicted for larger friction coefficients. Again, detailed examination of the frictional mechanics indicates a complex response. Gross slip occurs along the contact surface for approximately $0 \leq \mu \leq 0.05$ resulting in the rapid increase in dissipation. Slip-stick reversal occurs for approximately $0.05<\mu \leq \mu_{c}=0.3$, where the increase in dissipation results from larger $\mu$ even 
Case $\mathbf{F}_{1}$
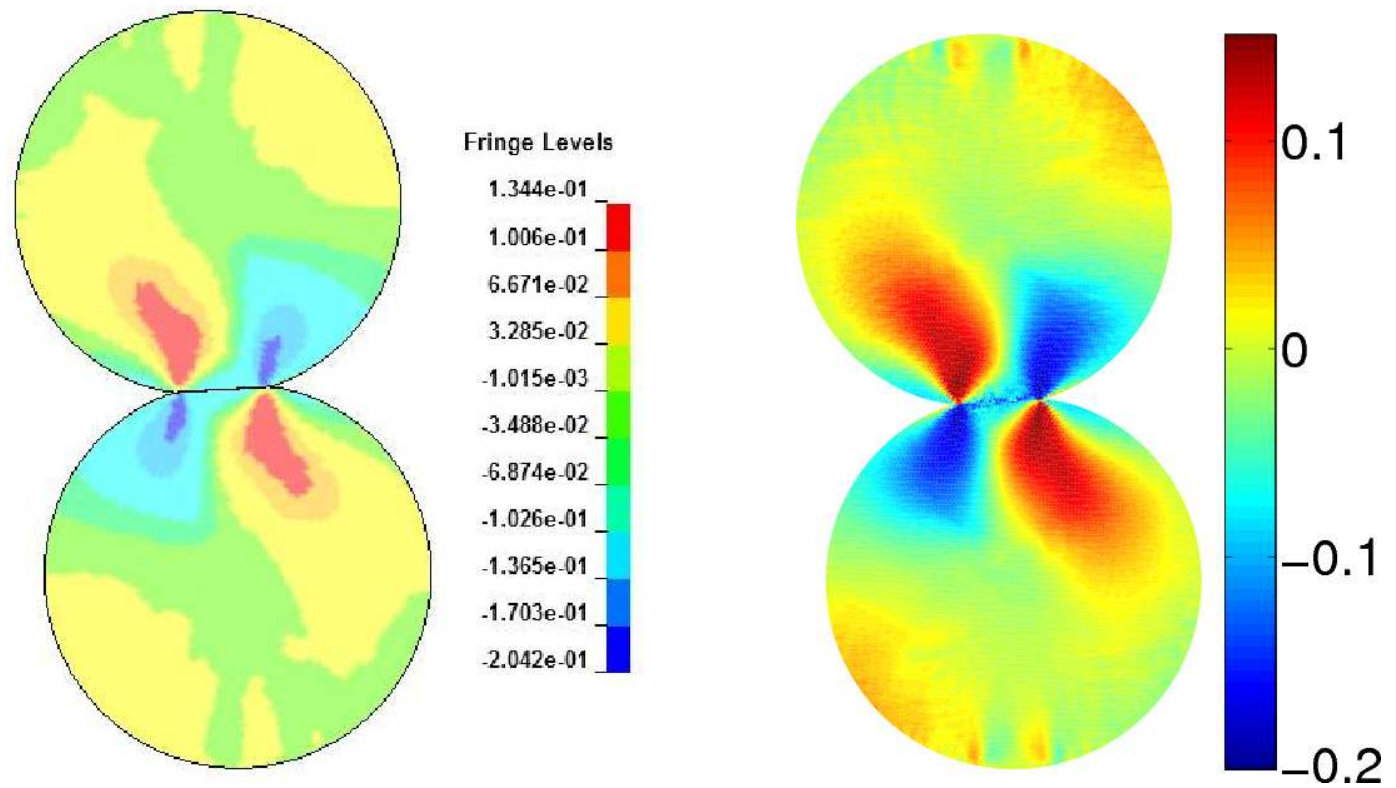

Case $\mathbf{F}_{2}$

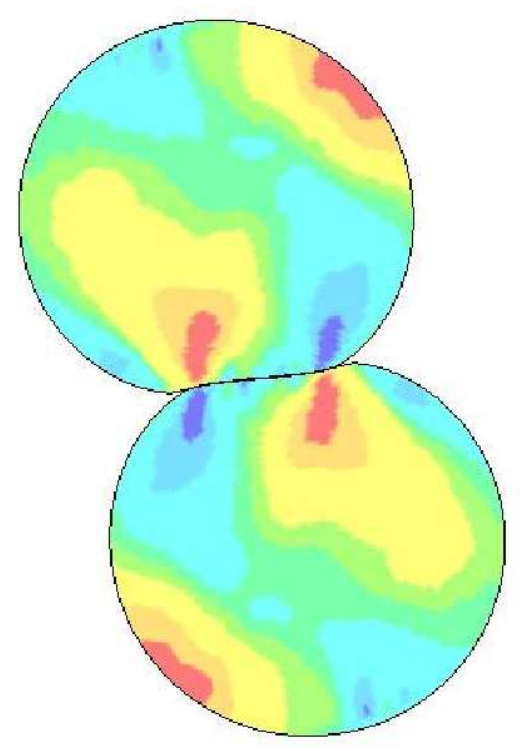

(a)

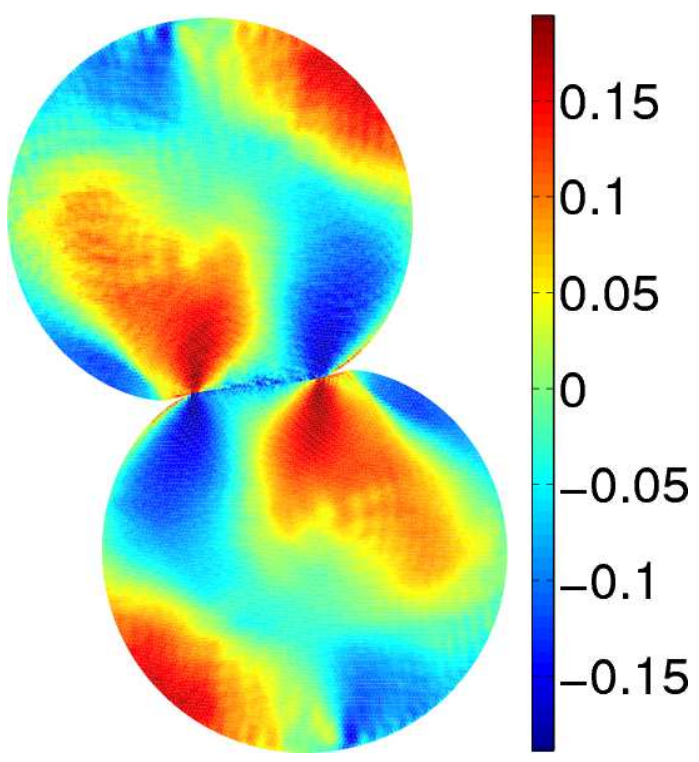

(b)

Figure 4.10: Spatial contours of in-plane shear stress : (a) Ls-Dyna and (b) FDEM. All values shown in the color-bar are in GPa. 
as stick becomes increasingly important. Frictional dissipation subsequently decreases for $\mu>\mu_{c}$ due to significant stick along the contact surface. Clearly, from the Fig. 4.11(b), the FDEM results and the Ls-Dyna results match very well.

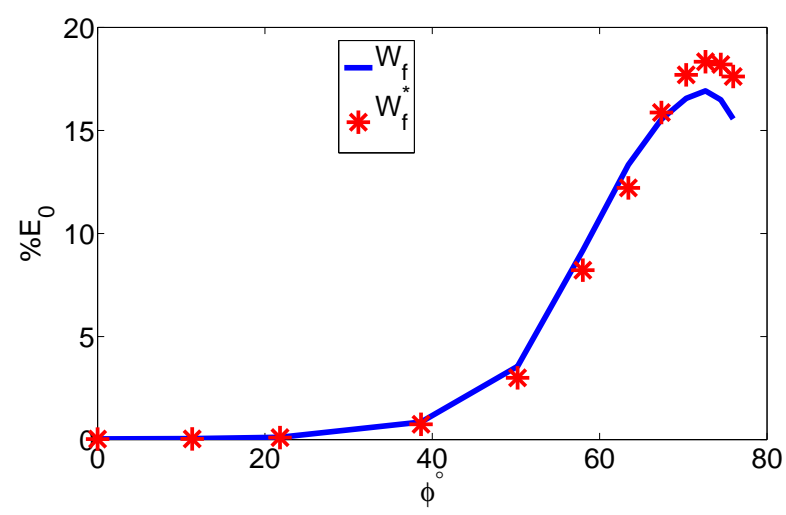

(a)

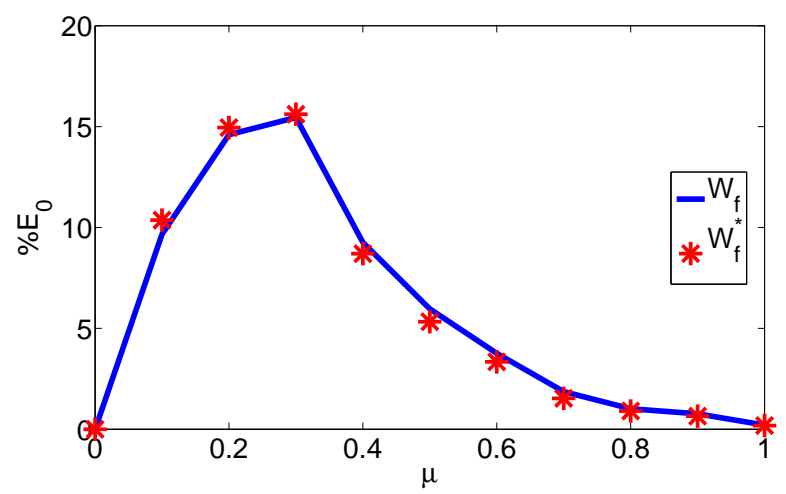

(b)

Figure 4.11: Variation in frictional dissipation with respect to (a) impact angle and (b) friction coefficient.

Spatial and temporal convergence of the FDEM is established for the elastic-viscoplasticfrictional with $v_{0}^{n}=300 \mathrm{~m} / \mathrm{s}$ and $v_{0}^{t}=300 \mathrm{~m} / \mathrm{s}$. To establish spatial convergence, the particle is discretized using finite element edge lengths of $\Delta h=1,2,3,4$ and $7.25 \mu \mathrm{m}$, corresponding to $71882,17803,7845,4373$ and 1385 finite elements per body, respectively. A constant time increment of $\Delta t=0.01 \mathrm{~ns}$ is used for all the simulations. The percent relative error, $\epsilon$, for the elastic-viscoplastic-frictional case is computed for each simulation based on the predicted coefficient of restitution, $e$, during impact: $\epsilon=|e-\hat{e}| / \hat{e} \times 100 \%$. Here, $\hat{e}$ is the numerical estimate predicted by the most resolved simulation. The results are summarized on the $\ln (\Delta h)-\ln (\epsilon)$ plot in Fig. 4.17 (a). The numerical method is shown to have close to quadratic spatial convergence based on a least squares linear fit of the data. To establish the temporal convergence, the time domain is discretized using time intervals $\Delta t=0.01,0.02,0.04$ and $0.08 \mathrm{~ns}$. The spatial domain is discretized using $\Delta h=2 \mu \mathrm{m}$. Here, for each simulation, $\epsilon$ is computed in the same manner as the spatial convergence study with $\hat{e}$ representing the numerical estimate predicted for the case $\Delta t=0.01 \mathrm{~ns}$. The results are summarized on the $\ln (\Delta t)-\ln (\epsilon)$ plot in Fig. 4.17(b). The numerical method is 


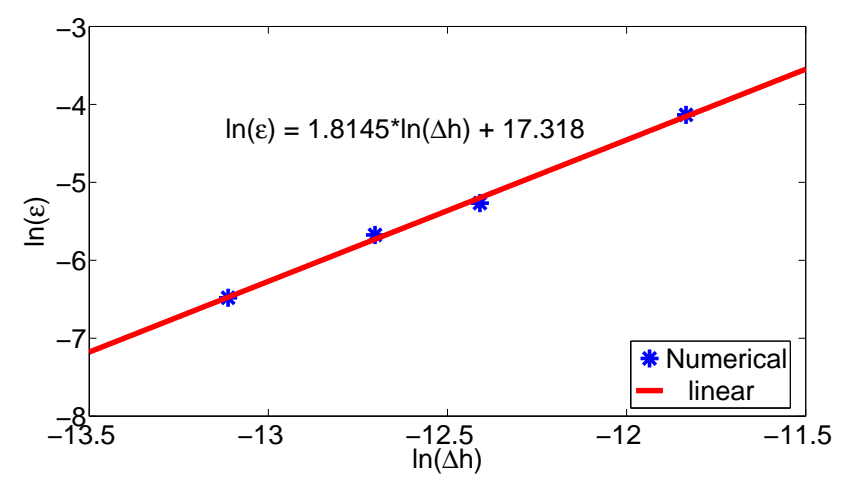

(a)

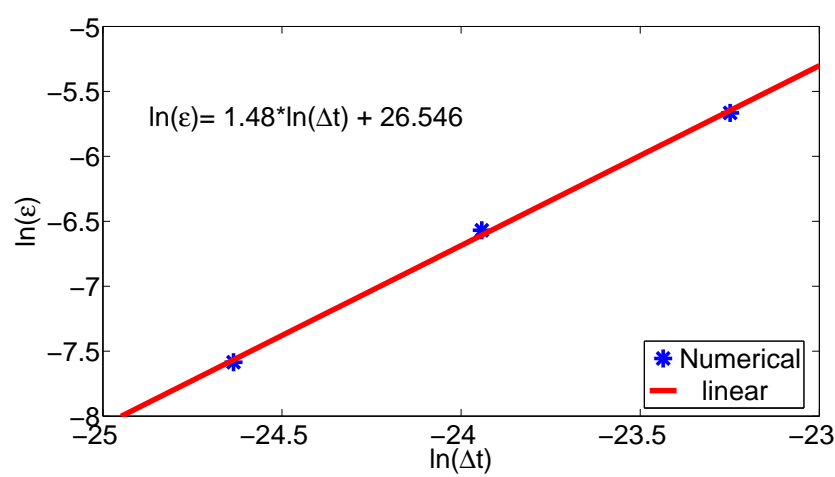

(b)

Figure 4.12: Convergence study for (a) elastic-viscoplastic impact and (b) elastic-viscoplasticfrictional impact.

shown to have close to quadratic temporal convergence based on a least squares linear fit of the data.

\subsubsection{Sensitivity to Penalty Parameter}

Numerical predictions obtained using the combined finite discrete element method are sensitive to the choice of the penalty parameter. Both the normal and the tangential penalty parameter, can change the kinematics of impact and subsequently the energetics. Therefore, different penalty values can lead to completely different results. The key idea in choosing the right penalty parameter is to make it large enough to limit penetration and minimize residual errors while avoiding numerical instabilities. Recognizing the importance of the penalty parameter, it is instructive to characterize the sensitivity of the numerical predictions to variations in penalty parameter values.

To this end, the test case of elastic-viscoplastic-frictional impact with $v_{0}^{n}=v_{0}^{t}=300 \mathrm{~m} / \mathrm{s}$ is considered. Figures 4.13 and 4.14 show the sensitivity of the kinematics and energetics to $p e^{n}$ and $p e^{t}$, respectively. Figures $4.13(\mathrm{a})$ and $4.14(\mathrm{a})$ show the variation in final plastic work $W_{p}$ and friction work $W_{f}$, given as a percentage of total initial energy $E_{0}$, with $p e^{n}$ and $p e^{t}$. Figures 4.13(b) and 4.14(b) show the variation in distance separating the particle center of masses at fixed time with $p e^{n}$ and $p e^{t}$; these plots are intended to highlight how the kinematics is affected by the penalty parameters. 
For the predictions shown in Fig. 4.13, the value of the tangential penalty parameter is fixed at $p e^{t}=1 \times 10^{11}$. Large penetration occurs for approximately $p e^{n} \leq 24 \times 10^{8}$ resulting in inaccurate stress and strain fields, whereas numerical instabilities occur for $p e^{n} \geq 120 \times 10^{9}$ resulting in spurious predictions. For the predictions shown in Fig. 4.14, the value of the normal penalty parameter is fixed at $p e^{n}=24 \times 10^{9}$. Essentially frictionless impact results for approximately $p e^{t} \leq 1 \times 10^{10}$, whereas numerical instabilities again develop for $p e^{t} \geq 1 \times 10^{12}$. Minimal variation in $W_{p}, W_{f}$, and $d_{c m}$ is predicted between these critical ranges of values. Based on these predictions, in this study $p e^{n}=24 \times 10^{9}$ and $p e^{t}=1 \times 10^{11}$ is used for all the simulations.

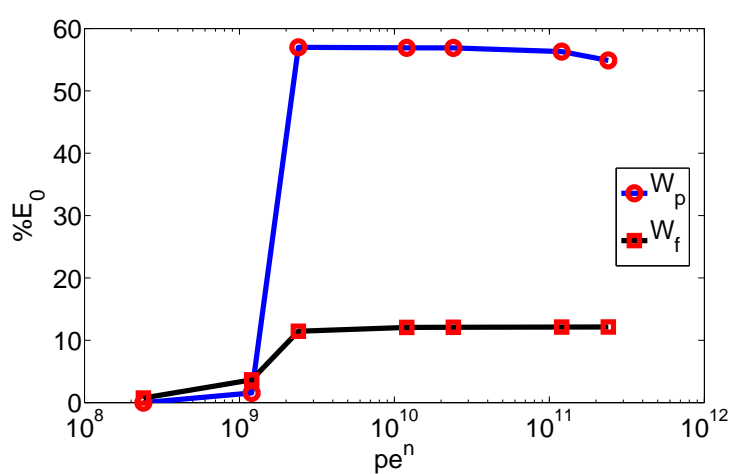

(a)

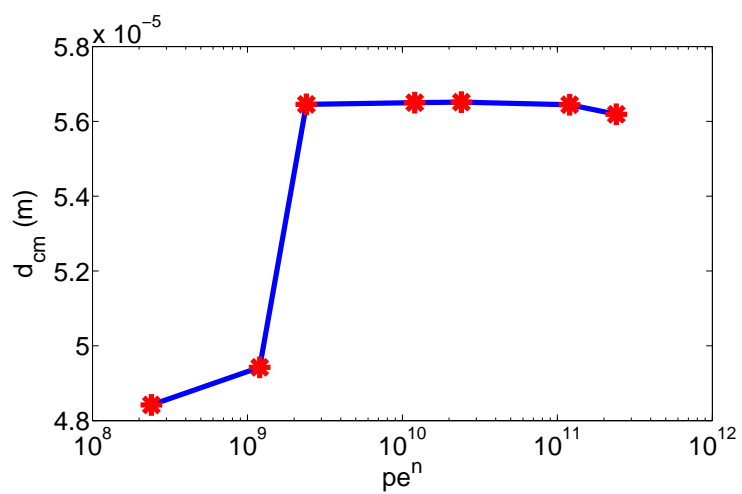

(b)

Figure 4.13: Sensitivity to the normal penalty parameter: (a) Variation in plastic and friction work. (b) Variation in distance separating the center of masses of the two bodies at fixed time.

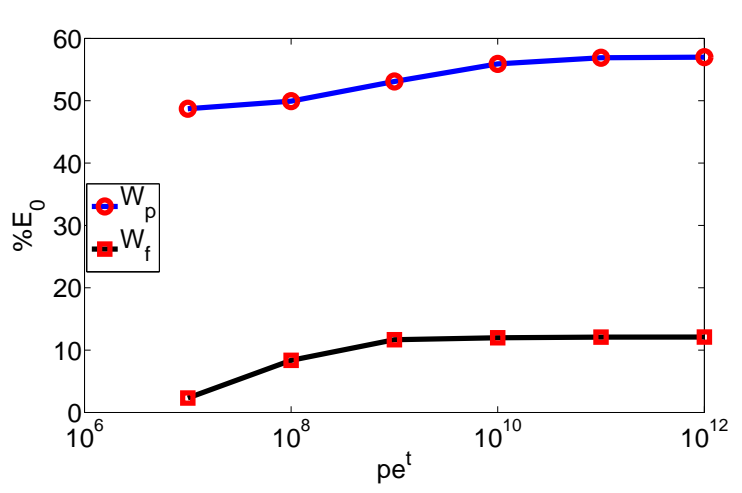

(a)

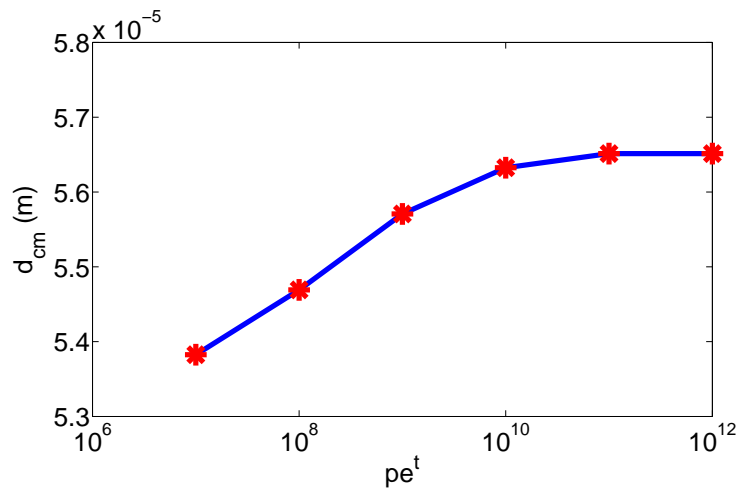

(b)

Figure 4.14: Sensitivity to the tangential penalty parameter: (a) Variation in plastic and friction work. (b) Variation in distance separating the center of masses of the two bodies at fixed time. 


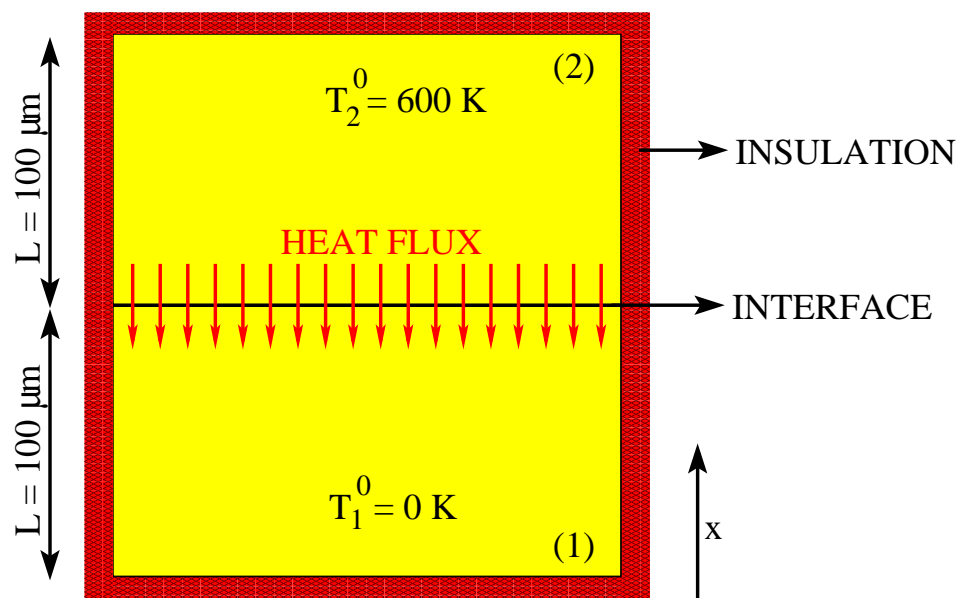

(a)

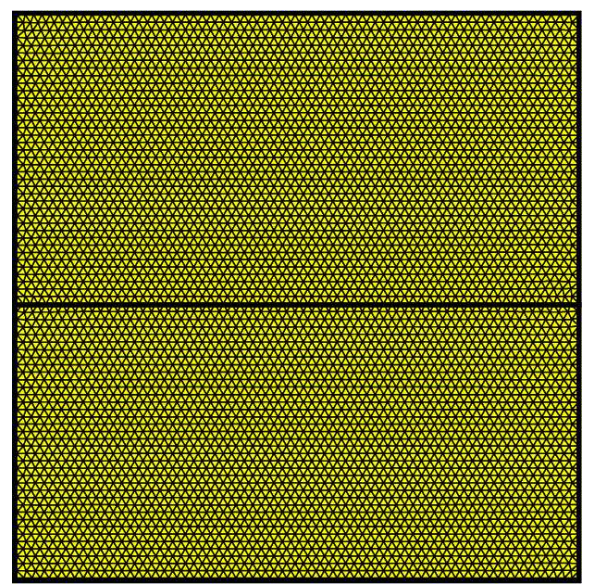

(b)

Figure 4.15: Initial configuration for thermal contact of rectangular blocks (a) Continuous model. (b) Discretized model.

\subsection{Verification: Thermal Contact Constraints}

Comparisons are given in this section between the numerical prediction and known analytical solution of ideal thermal contact between two rectangular blocks with different initial temperatures. The initial configuration for this test case is shown in Fig. 4.15(a). The two blocks, labeled as block (1) and block (2), of size $L \times 2 L$, where $L=100 \mu m$, have identical material properties, which are given in Table 4.1. The value for the thermal conductivity chosen in this study is not representative of HMX. A rather large value of $k_{T}=0.5 \times 10^{5} \mathrm{~W} / \mathrm{m}-K$ is chosen to examine the effects of thermal diffusion within the computational time domain. Blocks (1) and (2), which have uniform initial temperatures $T_{1}^{0}=600 \mathrm{~K}$ and $T_{2}^{0}=0 \mathrm{~K}$, respectively, are brought into contact at time $t=0$. Both blocks are insulated on all sides except on the side where contact occurs. When contact occurs, the temperatures at the contact interface instantly equilibriate to the steady state temperature. With time, heat flows from the hotter block to the cooler block until both blocks attain steady state temperature.

Before comparing the analytical and numerical predictions, the analytical solution to the problem is briefly described. For the problem at hand, the steady state temperature for the two blocks is simply given by $T_{s}=0.5\left(T_{1}^{0}+T_{2}^{0}\right)$. Due to symmetry, the problem reduces to a $1 \mathrm{D}$ transient heat conduction problem whose solution is obtained by solving the partial differential 
equation (PDE):

$$
\frac{\partial^{2} T_{a}}{\partial x^{2}}=\frac{\rho_{0} c_{v}}{k_{T}} \frac{\partial T_{a}}{\partial t}
$$

subject to initial conditions:

$$
T_{a}(x, 0)=T_{1}^{0} \text { for block (1), } \quad T_{a}(x, 0)=T_{2}^{0} \text { for block }(2) .
$$

The boundary conditions for block (1) are given by

$$
\frac{\partial T_{a}}{\partial x}=0 \text { at } x=0, \quad T_{a}=T_{s} \text { at } x=L .
$$

The boundary conditions for block (2) are given by

$$
\frac{\partial T_{a}}{\partial x}=0 \text { at } x=2 L, \quad T_{a}=T_{s} \text { at } x=L .
$$

The second boundary condition in Eqs. (4.7) and (4.8) represents the ideal thermal contact boundary conditions. The analytical solution to the above problem is given by

$$
T_{a}(x, t)=T_{s}+\sum_{m=0}^{\infty} e^{-\alpha t \lambda_{m}^{2}} A_{m} \cos \left(\lambda_{m} x\right),
$$

where coefficients $\lambda_{m}$ and $A_{m}$ are given by

$$
\lambda_{m}=\frac{m \pi}{2 L}, \quad A_{m}=\frac{2 \sin \left(\lambda_{m} L\right)\left(T_{1}^{0}-T_{2}^{0}\right)}{m \pi} .
$$

Numerical solution to the problem is obtained by solving the energy equation using the combined discrete finite element method on the discretized configuration as shown in Fig. 4.15(b), where the plane surface of each block is discretized into 5020 finite elements comprising of 2615 nodes.

Comparisons between the numerical and analytical solutions are shown in Fig. 4.16. Figure 4.16(a) shows the variation in the maximum and minimum temperatures within the two blocks 
with respect to time. At time $\mathrm{t}=0$, the maximum and minimum temperatures correspond to the initial temperatures of the two blocks. With time, block (1) loses heat and block (2) gains heat such that both bodies approach the steady state temperature at end time. Figure 4.16(b) shows the spatial variation of temperature within the two blocks after 500, 1000 and 1500 ns. The temperature at the interface approaches the steady state temperature at a rate governed by the thermal penalty parameter. Ideal thermal contact conditions are enforced for $p e^{t h} \rightarrow \infty$. However, large penalty values lead to numerical instabilities. The sensitivity of the predictions to the thermal penalty parameter is demonstrated later. From the figure it is evident that the numerical predictions and the analytical results agree very well.

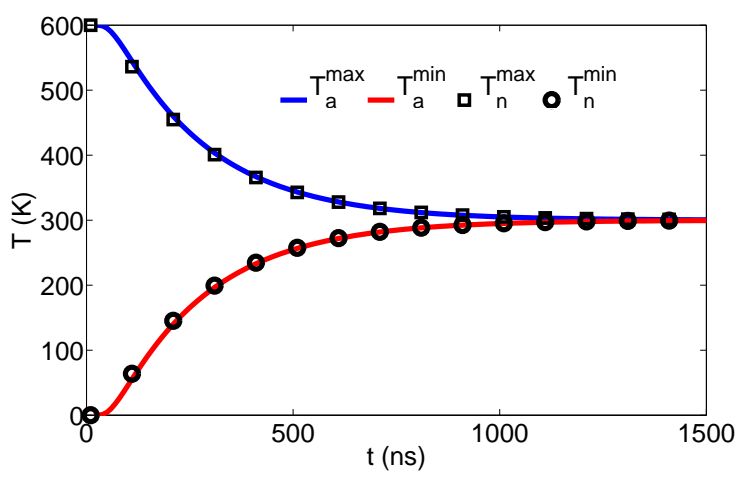

(a)

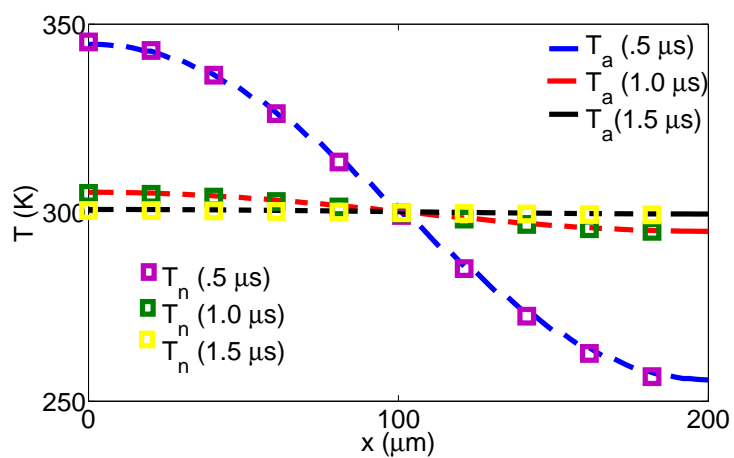

(b)

Figure 4.16: (a) Temporal variation of the maximum and minimum temperatures within the blocks: Analytical and numerical predictions. (b) Spatial variation of temperature after $500 \mathrm{~ns}$, $1000 \mathrm{~ns}$ and $1500 \mathrm{~ns}$ : Analytical and numerical predictions.

Convergence of the FDEM is established for the ideal thermal contact test case with $T_{1}^{0}=$ $600 \mathrm{~K}$ and $T_{2}^{0}=0 \mathrm{~K}$. To this end, the plane surface of the blocks is discretized using finite element edge lengths of $\Delta h=2,3,7$ and $10 \mu \mathrm{m}$, corresponding to 5020, 3773, 545 and 305 finite elements per body, respectively. The percent relative error, $\epsilon$, is computed for each simulation based on the predicted time required for the blocks to reach steady state behavior, $t_{f}, \epsilon=\left|t_{f}-\hat{t}_{f}\right| / \hat{t}_{f} \times 100 \%$. Here, $\hat{t}_{f}$ is the analytical solution. The results are summarized on the $\ln (\Delta h)-\ln (\epsilon)$ plot in Fig. 4.17 (a). The numerical method is shown to have close to quadratic convergence based on a least squares linear fit of the data.

Sensitivity to the thermal penalty parameter is demonstrated by varying the thermal penalty 


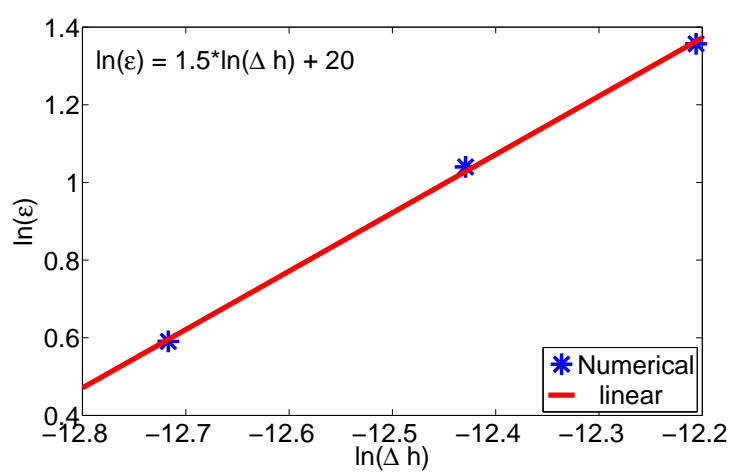

(a)

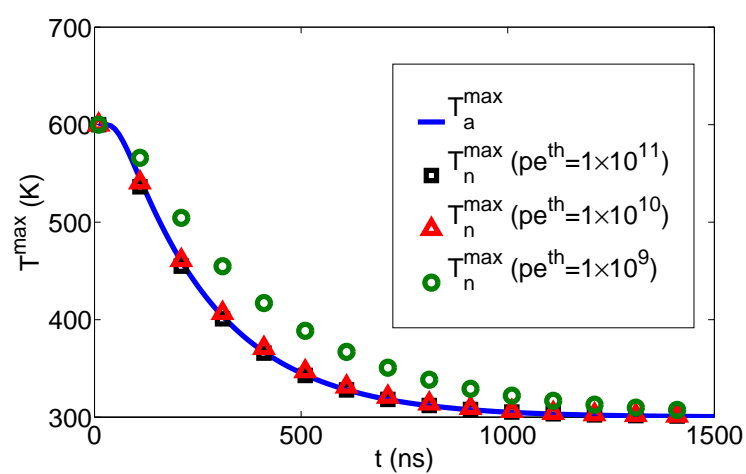

(b)

Figure 4.17: (a) Convergence study for thermal contact problem. (b) Sensitivity to the thermal penalty parameter: Variation of the temporal evolution of the maximum temperatures within the blocks.

parameter for the problem at hand. To this end, $p e^{t h}$ is varied from $1 \times 10^{9}$ to $1 \times 10^{11}$. The numerical predictions for the evolution of the maximum temperature within the 2 blocks along with the analytical result are shown in Fig. 4.17 (b). The results indicate that for large penalty values the thermal contact constraints are enforced more rigorously, therefore the numerical predictions closely approximate the analytical solution. Based on these predictions, in this study $p e^{t h}=$ $1 \times 10^{11}$ is used for all the simulations. 


\section{Chapter 5}

\section{Micro-Particle Cluster Impact}

Predictions are given in this chapter for the simulation of inert, plane strain (2-D), particle scale impact occurring in the vicinity of a rigid, planar impactor surface, as illustrated in Fig. 5.1. Emphasis is placed on characterizing the variation in temporal and spatial partitioning of energy within the material with impact angle $\phi^{\circ}$, and on identifying the relative importance of plastic and friction work as potential hot-spot formation mechanisms. Rather than modeling the configuration shown in Fig. 5.1(a), an equivalent configuration shown in Fig. 5.1(b) is addressed, where the particles impact a stationary, rigid wall at uniform speed and angle. For tractability, attention is focused on describing the response of a small, close, randomly packed, and numerically wellresolved micro-particle cluster $(\approx 25$ particles). This simpler problem retains important features of the more general problem, and provides a rational foundation for additional study on the influence of far-field particle interactions (i.e., particle self-confinement) and combustion on the 3-D near wall impact response.

This analysis is important for the following reasons. First, the impact angle will always vary for loading events in practice, and locally depends on impactor geometry. Second, bulk experiments indicate that combustion of energetic solids often first occurs near the impactor surface, implying

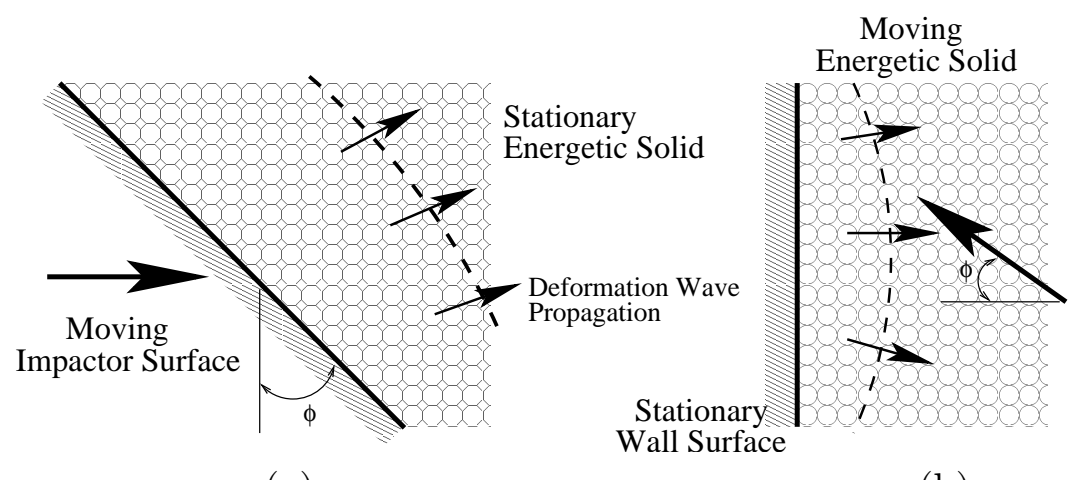

(a)

Figure 5.1: Schematic of a (a) moving impactor and stationary energetic solid and (b) stationary wall and moving energetic solid. 
that particle heating is largest at that location. Third, uncertainty exists about the coupled effects of particle-piston and particle-particle frictional interactions on stress and energy states within dynamically loaded granular materials and how they can facilitate reactive hot-spot formation. Last, the development of suitable multi-dimensional boundary conditions for use with bulk scale models requires a firm understanding of particle scale physics occurring in the immediate vicinity of solid boundaries.

An outline of this chapter is as follows. First, predictions for both normal and oblique impact of a micro-particle cluster with a stationary, planar, rigid wall are presented in Section 5.1. Emphasis is placed on examining the temporal and spatial partitioning of cluster energy. Particular emphasis is placed on estimating the fraction of cluster mass that is heated to elevated temperature because of its relevance to combustion initiation. Sensitivity of the predictions to the initial particle packing configuration is presented in Section 5.2.

\subsection{Cluster Impact}

The numerical model described and verified in the previous chapters is used to simulate the impact of a micro-particle cluster with a stationary, rigid planar wall. Emphasis is placed on characterizing how viscoplastic deformation, friction, impact angle, and cluster configuration affect the spatial and temporal partitioning of cluster energy. For compactness, the notation $\mathbf{P} . \phi^{\circ}$ is used to represent elastic-viscoplastic-frictionless impact cases, where $\phi^{\circ}$ is the impact angle expressed in degrees; likewise, the notation $\mathbf{F} . \phi^{\circ}$ is used to represent elastic-viscoplastic-frictional impact cases. The initial close-packed cluster configuration used for these cases is shown in Fig. 5.2(a); it consists of 25 well-resolved particles, where each particle has an initial radius of $50 \mu \mathrm{m}$, and contains $N_{e}=1033$ finite elements and $N_{n}=567$ nodes. Its initial speed is fixed at $300 \mathrm{~m} / \mathrm{s}$ for all cases. Though this specific cluster configuration and initial speed are used here, variations in these quantities, and in particle size, will affect impact energetics. To demonstrate the influence of initial cluster configuration on the energetics of elastic-viscoplastic-frictional impact, several simulations are also performed for the configurations shown in Fig. 5.2(b-d), where the initial 


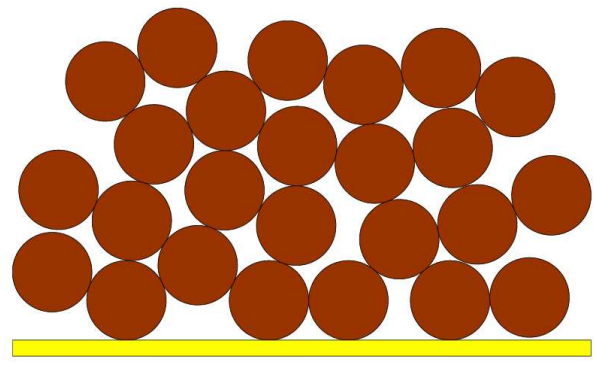

(a)

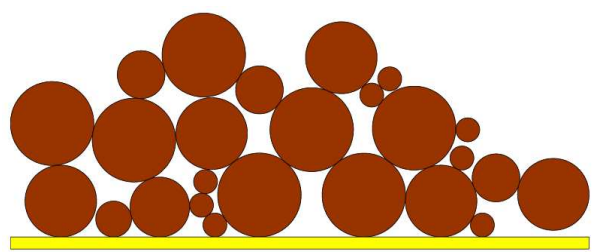

(c)

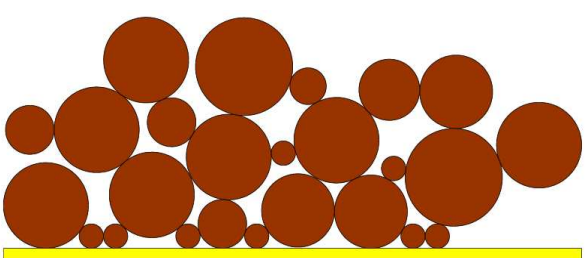

(b)

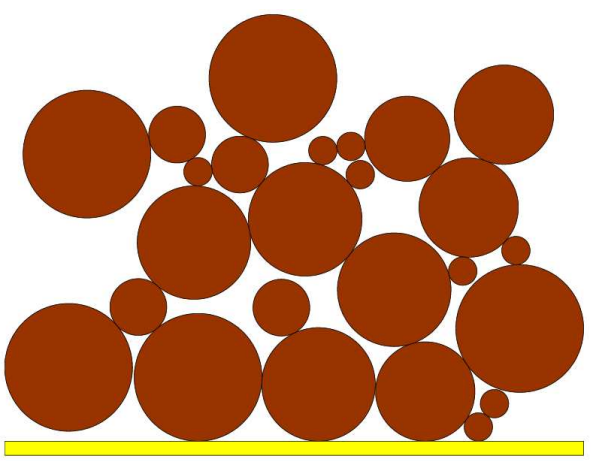

(d)

Figure 5.2: Initial particle packing configurations used in this study: (a) Configuration 1, (b) Configuration 2, (c) Configuration 3, and (d) Configuration 4.

cluster speeds are the same as those of configuration 1. The cluster mass and initial energy for all configurations are listed in Table 5.1. Also listed in this table are values for mean particle radius $(\bar{R})$, standard deviation of particle radius $(\widetilde{R})$, and initial solid volume fraction (SVF) for all configurations. The initial solid volume fraction is computed as the ratio of the total particle area and the area of the smallest rectangle that encompasses all particles at time $t=0$. Values for the material and numerical parameters are listed in Table 4.1. The numerical algorithm, implemented using FORTRAN 90, was executed on a 2.66 GHz Dual Core Xeon 64-bit workstation having 4 GB RAM. A typical run time for a single simulation was approximately one hour.

The principal concern of this analysis is to describe the short time response of the particle cluster immediately following initial impact when dissipation is most significant. Before focusing on this response, however, the longer time response is briefly illustrated for case $\mathbf{F . 0}{ }^{\circ}$ in Fig. 5.3. Shown in this figure are the spatial contours of kinetic energy at several times up to $1 \mu \mathrm{s}$ following initial impact. Particles closest to the wall experience a rapid decrease in kinetic energy 
$100 \mathrm{~ns}$

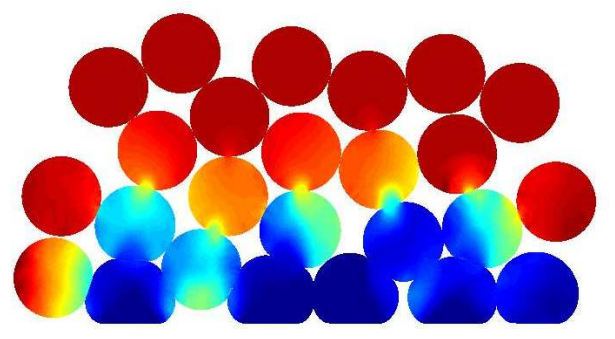

$300 \mathrm{~ns}$

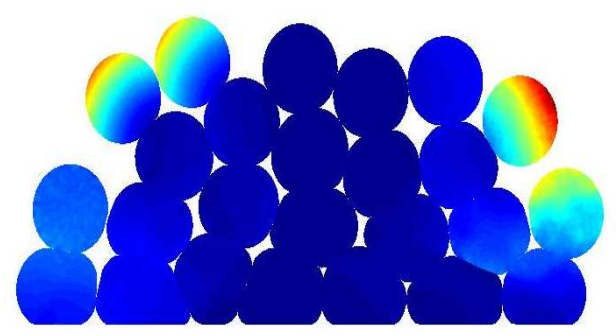

$500 \mathrm{~ns}$

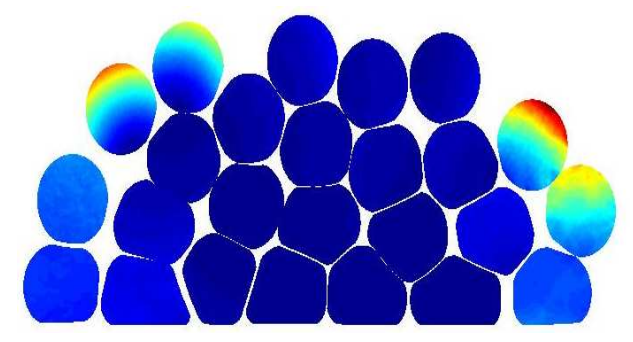

$700 \mathrm{~ns}$

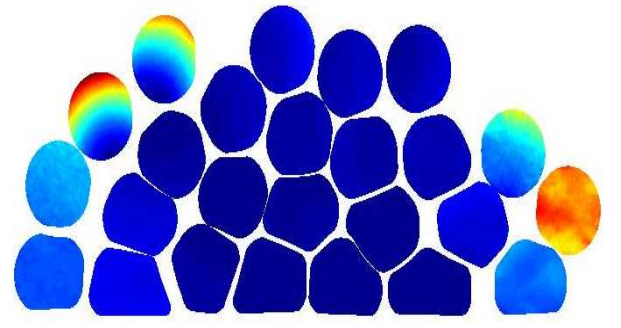

$900 \mathrm{~ns}$

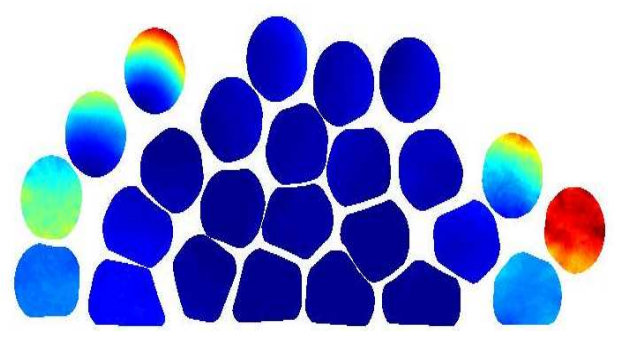

$200 \mathrm{~ns}$

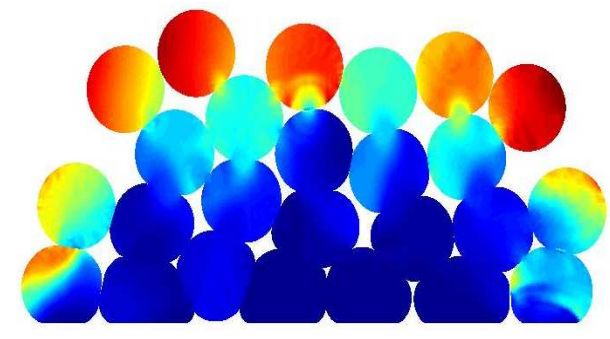

$400 \mathrm{~ns}$

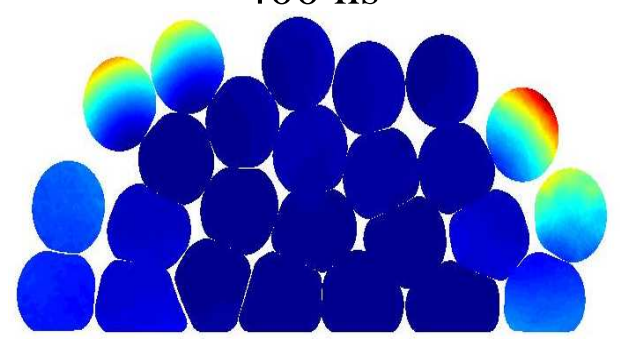

$600 \mathrm{~ns}$

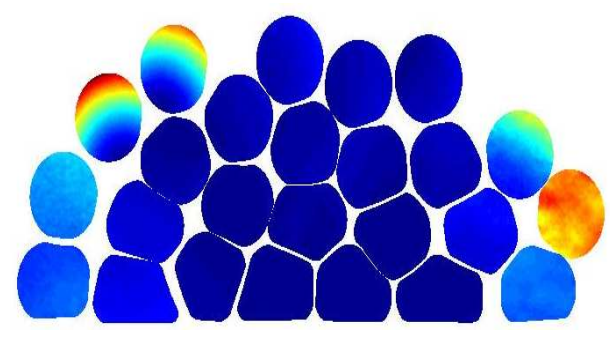

$800 \mathrm{~ns}$

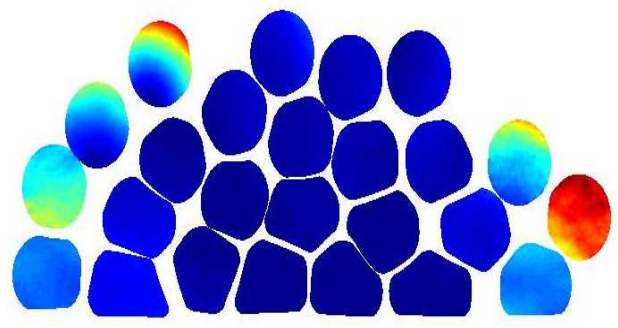

$1000 \mathrm{~ns}$

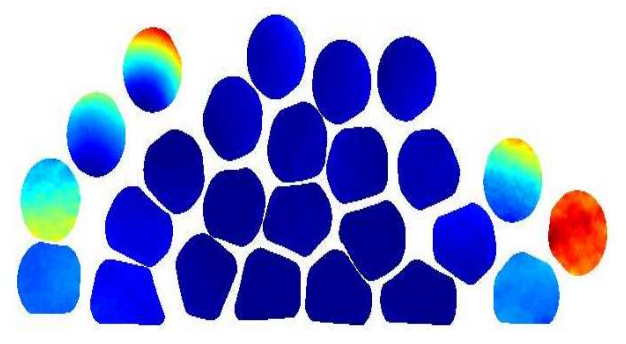

Figure 5.3: Predicted particle positions and kinetic energy contours for case $\mathbf{F} . \mathbf{0}^{\circ}$. 
Table 5.1: Cluster mass and initial energy for all the configurations.

\begin{tabular}{cccccc}
\hline \hline Configuration & $\mathcal{B}(\mathrm{Kg} / \mathrm{m})$ & $E_{0}(\mathrm{~J} / \mathrm{m})$ & $\bar{R}(\mu \mathrm{m})$ & $\widetilde{R}(\mu \mathrm{m})$ & $S V F$ \\
\hline \hline 1 & $3.7338 \times 10^{-4}$ & 16.8022 & 50.0 & 0 & $64.6 \%$ \\
2 & $4.1404 \times 10^{-4}$ & 18.6318 & 47.6 & 22.96 & $60.5 \%$ \\
3 & $3.8057 \times 10^{-4}$ & 17.1257 & 46.0 & 21.21 & $55.1 \%$ \\
4 & $5.6170 \times 10^{-4}$ & 25.2765 & 54.4 & 28.88 & $60.2 \%$ \\
\hline \hline
\end{tabular}

upon impact that is accompanied by significant viscoplastic deformation. A deformation wave subsequently diffracts away from the wall through inter-particle contact, affecting the stress and energy of particles located within the cluster and along its periphery. The deformation, stress, and energy fields within particles located along the wall continues to evolve due to repeated impact by adjacent particles until the cluster is dispersed at later time due to a loss of peripheral confinement. Larger close-packed particle ensembles $(N>100)$ may result in a significantly different near wall response than predicted here due to the influence of far-field confinement; this issue is addressed in the next chapter. Because predictions indicate that much of the dissipation occurs close to the wall shortly after initial impact, the remainder of this analysis is restricted to the early cluster response for $0 \leq t \leq 300 \mathrm{~ns}$.

\subsubsection{Cluster Energetics}

Figure 5.4 gives predicted history profiles for the partitioning of total cluster energy $\left(E_{T}\right)$ into potential $\left(W_{e}\right)$, kinetic $\left(E_{k}\right)$, plastic $\left(W_{p}\right)$, and frictional $\left(W_{f}\right)$ components for the representative cases $\mathbf{P . 0}{ }^{\circ}, \mathbf{P . 3 0}{ }^{\circ}, \mathbf{P . 6 0}{ }^{\circ}, \mathbf{F} .0^{\circ}, \mathbf{F} . \mathbf{3 0}^{\circ}$ and $\mathbf{F} .60^{\circ}$. These predictions, which highlight the influence of viscoplastic deformation, friction, and impact angle on cluster energetics, are qualitatively similar to the verification predictions for elastic-plastic-frictional impact shown in the previous chapter, in that exchange between kinetic energy and plastic work dominates the early time response. Weak oscillations are observed in potential and kinetic energy due to successive impact and release between particles. The energy components approach nearly uniform values at $t=$ $300 \mathrm{~ns}$ as particles disperse and lose contact. Values for the final partitioning of total cluster energy for each of these cases, and other impact cases, are summarized in Tables 5.2 and 5.3. 


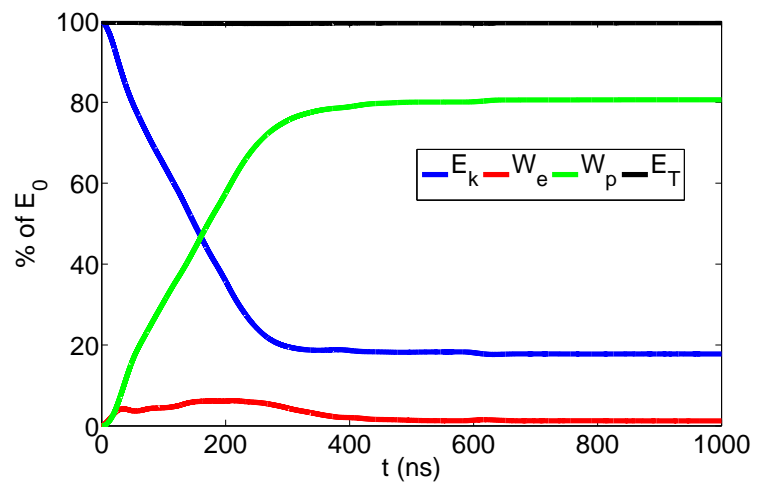

$\left(\mathbf{P} .0^{\circ}\right)$

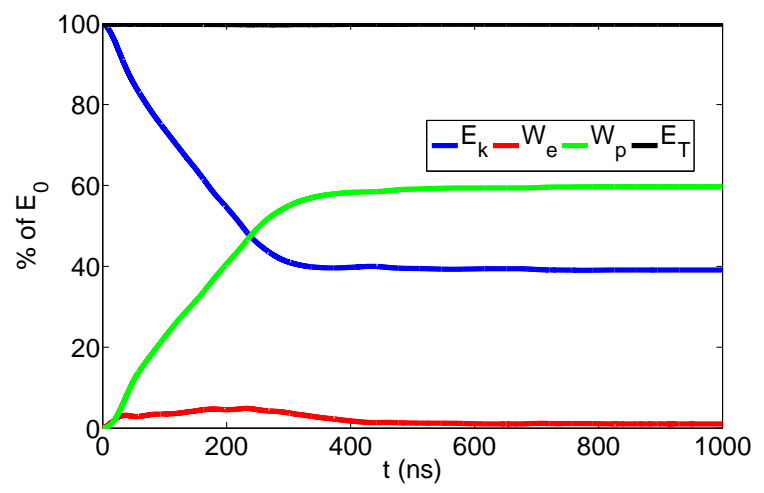

$\left(\mathrm{P} .30^{\circ}\right)$

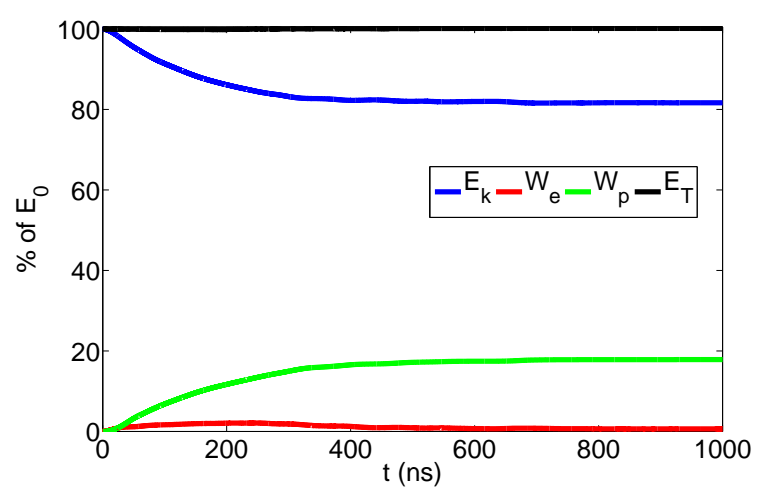

$\left(\mathbf{P . 6 0} 0^{\circ}\right)$

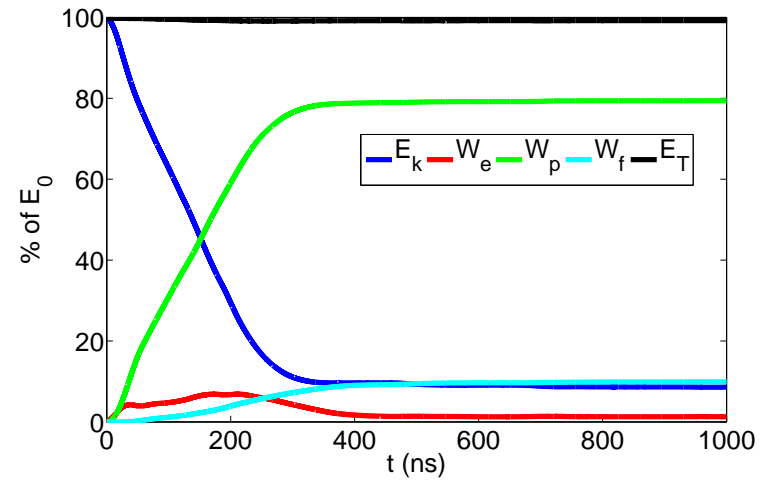

$\left(\mathbf{F} .0^{\circ}\right)$

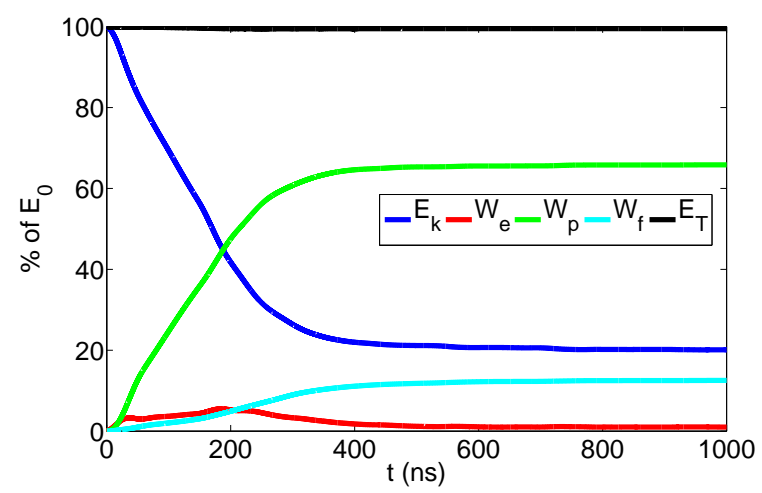

$\left(\mathrm{F} .30^{\circ}\right)$

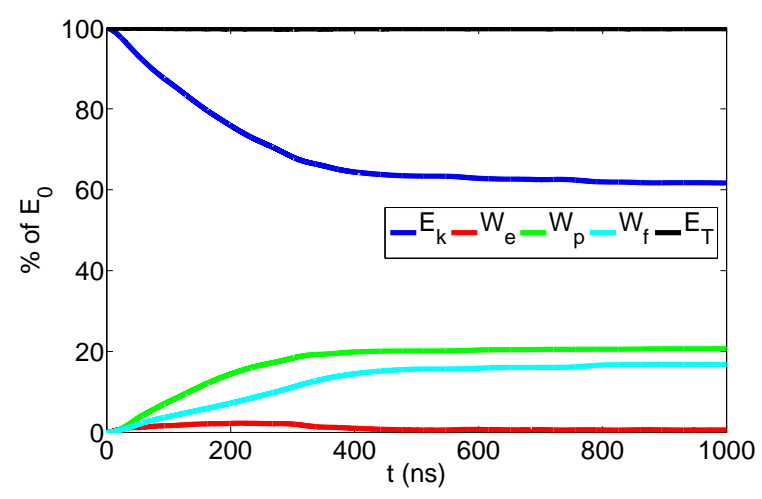

$\left(\mathbf{F} .60^{\circ}\right)$

Figure 5.4: Predictions for the partitioning of total cluster energy. 
Comparing results for cases $\mathbf{P} . \mathbf{0}^{\circ}-\mathbf{P} . \mathbf{8 0} 0^{\circ}$ with those for cases $\mathbf{F} . \mathbf{0}^{\circ}-\mathbf{F} . \mathbf{8 0}^{\circ}$ shows that the potential energy and plastic work histories are both qualitatively and quantitatively similar indicating that they are minimally influenced by friction. Indeed, the final potential energy and plastic work values for cases $\mathbf{P} . \mathbf{0}^{\circ}-\mathbf{P} . \mathbf{8 0}^{\circ}$ are only marginally higher $(<2.48 \%)$ than those for cases $\mathbf{F} . \mathbf{0}^{\circ} \mathbf{- F} . \mathbf{8 0}^{\circ}$. As with the verification predictions, the major effect of friction is to significantly reduce cluster kinetic energy as time evolves. The final kinetic energy for case $\mathbf{P . 0}{ }^{\circ}$ is approximately twice that of case $\mathbf{F} . \mathbf{0}^{\circ}$, and similar differences are predicted between cases $\mathbf{P . 1 5}{ }^{\circ}-\mathbf{P . 8 0}$ and the corresponding cases $\mathbf{F} . \mathbf{1 5}^{\circ} \mathbf{- F} \cdot \mathbf{8 0}^{\circ}$. Plastic work is largest for normal impact, and consists of $80.63 \%$ and $79.91 \%$ of the initial total energy for cases $\mathbf{P . 0 ^ { \circ }}$ and $\mathbf{F} . \mathbf{0}^{\circ}$, respectively. Because plastic work is primarily driven by the normal component of cluster velocity, it monotonically decreases with impact angle. The percentage of friction work increases with impact angle to a maximum value of $18.03 \%$ for $\phi_{c}^{\circ}=50^{\circ}$, and then decreases for impact angles greater than this value. This trend is similar to the results shown in Fig. 4.11(a) for transverse impact of two identical cylinders in plane strain, though the peak percentage of frictionally dissipated energy occurs at a higher impact angle as the kinematics of multiparticle impact are drastically different from the transverse impact cases. However, a higher percentage of frictionally dissipated energy does result for normal cluster impact (case $\mathbf{F} . \mathbf{0}^{\circ}$ ) than for two particle impact due to interactions between particles near the wall. Importantly, this result suggests that considerable frictional heating may occur near penetrator surfaces even for normal impact.

Also given in this table is the numerical error between the predicted final total energy and the exact value $E_{0}=16.8022 \mathrm{~J} / \mathrm{m}$. The numerical error is less than $0.34 \%$ of $E_{0}$ for all cases studied, and is slightly larger for the normal impact cases due to higher induced stresses and inter-particle penetration. Because inter-particle penetration is directly proportional to the normal component of particle velocity, the numerical error reduces with increasing $\phi^{\circ}$.

The variation in the bulk partitioning of energy with position normal to the wall, is now considered. Defining $d m \equiv \rho \psi d A$ in Eq. (2.50), where $\psi=\psi(\mathbf{x}, t)$ is a scalar material point function whose value equals unity within particles and vanishes elsewhere, $\mathbf{x}$ is position in the 
Table 5.2: Partition of total energy and numerical error for cluster Configuration 1.

\begin{tabular}{cccccc}
\hline \hline Case & $W_{p}$ & $W_{e}$ & $E_{k}$ & $W_{f}$ & $\epsilon$ \\
\hline \hline $\mathbf{P . 0}^{\circ}$ & $80.63 \%$ & $1.25 \%$ & $17.79 \%$ & - & $0.34 \%$ \\
$\mathbf{P . 5}^{\circ}$ & $80.73 \%$ & $1.23 \%$ & $18.48 \%$ & - & $0.3 \%$ \\
$\mathbf{P . 1 0}^{\circ}$ & $78.72 \%$ & $1.22 \%$ & $20.27 \%$ & - & $0.22 \%$ \\
$\mathbf{P . 1 5}^{\circ}$ & $75.48 \%$ & $1.19 \%$ & $23.38 \%$ & - & $0.05 \%$ \\
$\mathbf{P . 2 0}^{\circ}$ & $71.38 \%$ & $1.15 \%$ & $27.65 \%$ & - & $0.18 \%$ \\
$\mathbf{P . 2 5}^{\circ}$ & $66.28 \%$ & $1.11 \%$ & $32.89 \%$ & - & $0.28 \%$ \\
$\mathbf{P . 3 0}^{\circ}$ & $59.71 \%$ & $1.05 \%$ & $39.1 \%$ & - & $0.15 \%$ \\
$\mathbf{P . 3 5}^{\circ}$ & $52.96 \%$ & $0.99 \%$ & $45.96 \%$ & - & $0.09 \%$ \\
$\mathbf{P . 4 0}^{\circ}$ & $45.66 \%$ & $0.94 \%$ & $53.26 \%$ & - & $0.14 \%$ \\
$\mathbf{P . 4 5}^{\circ}$ & $38.38 \%$ & $0.84 \%$ & $60.69 \%$ & - & $0.08 \%$ \\
$\mathbf{P . 5 0}^{\circ}$ & $31.01 \%$ & $0.8 \%$ & $68.13 \%$ & - & $0.06 \%$ \\
$\mathbf{P . 5 5}^{\circ}$ & $24.1 \%$ & $0.7 \%$ & $75.19 \%$ & - & $0.02 \%$ \\
$\mathbf{P . 6 0}^{\circ}$ & $17.85 \%$ & $0.63 \%$ & $81.66 \%$ & - & $0.14 \%$ \\
$\mathbf{P . 6 5}^{\circ}$ & $12.22 \%$ & $0.58 \%$ & $87.39 \%$ & - & $0.19 \%$ \\
$\mathbf{P . 7 0}^{\circ}$ & $7.29 \%$ & $0.53 \%$ & $92.32 \%$ & - & $0.15 \%$ \\
$\mathbf{P . 7 5}^{\circ}$ & $3.67 \%$ & $0.31 \%$ & $96.11 \%$ & - & $0.09 \%$ \\
$\mathbf{P . 8 0}^{\circ}$ & $1.37 \%$ & $0.18 \%$ & $98.56 \%$ & - & $0.11 \%$ \\
\hline \hline
\end{tabular}

stationary, 2-D computational plane, and $d A=d y d x$ is a differential area element in this plane, then the plastic work in the direction normal to the wall for a transverse volume of width $\delta x$, centered at $x$, may be expressed by

$$
\bar{w}_{p}(x, t)=\frac{1}{\overline{\mathcal{B}}_{p}(x, t)} \int_{x-\frac{\delta x}{2}}^{x+\frac{\delta x}{2}} \int_{y_{1}}^{y_{2}} \int_{0}^{t} \psi \boldsymbol{\sigma}: \mathbf{d}_{p} d t^{\prime} d y^{\prime} d x^{\prime}
$$

where $y_{1}$ and $y_{2}$ are the lower and upper limits of the transverse section parallel to the wall and $\overline{\mathcal{B}}_{p}$ is the instantaneous cluster mass affected by plastic work within the transverse volume. Because friction is a surface phenomenon, friction work cannot be expressed on a per unit mass basis. However, for purposes of comparison with the mass specific plastic work, friction work is locally distributed to elements connected to the finite element nodes that are affected by the frictional heat flux. As such, the evolution of mass specific friction work for the cluster is described by

$$
\bar{w}_{f}(x, t)=\frac{1}{\overline{\mathcal{B}}_{f}(x, t)} \int_{x-\frac{\delta x}{2}}^{x+\frac{\delta x}{2}} \int_{y_{1}}^{y_{2}} \int_{0}^{t} \psi \dot{W}_{f} d t^{\prime} d y^{\prime} d x^{\prime}
$$


Table 5.3: Partition of total energy and numerical error for cluster Configuration 1.

\begin{tabular}{cccccc}
\hline \hline Case & $W_{p}$ & $W_{e}$ & $E_{k}$ & $W_{f}$ & $\epsilon$ \\
\hline \hline $\mathbf{F . 0}$ & $79.91 \%$ & $1.28 \%$ & $8.6 \%$ & $9.91 \%$ & $0.3 \%$ \\
$\mathbf{F . 5}^{\circ}$ & $79.81 \%$ & $1.27 \%$ & $8.99 \%$ & $9.65 \%$ & $0.28 \%$ \\
$\mathbf{F . 1 0}$ & $79.01 \%$ & $1.23 \%$ & $9.97 \%$ & $9.6 \%$ & $0.19 \%$ \\
$\mathbf{F . 1 5}$ & $77.36 \%$ & $1.19 \%$ & $11.55 \%$ & $9.71 \%$ & $0.19 \%$ \\
$\mathbf{F . 2 0}$ & $74.75 \%$ & $1.13 \%$ & $13.7 \%$ & $10.29 \%$ & $0.13 \%$ \\
$\mathbf{F . 2 5}$ & $71.1 \%$ & $1.09 \%$ & $16.5 \%$ & $11.2 \%$ & $0.11 \%$ \\
$\mathbf{F . 3 0}$ & $66.23 \%$ & $1.01 \%$ & $20.12 \%$ & $12.55 \%$ & $0.09 \%$ \\
$\mathbf{F . 3 5}$ & $59.63 \%$ & $0.95 \%$ & $25.02 \%$ & $14.35 \%$ & $0.05 \%$ \\
$\mathbf{F . 4 0}$ & $51.79 \%$ & $0.88 \%$ & $31.07 \%$ & $16.22 \%$ & $0.04 \%$ \\
$\mathbf{F . 4 5}$ & $43.66 \%$ & $0.82 \%$ & $37.95 \%$ & $17.53 \%$ & $0.04 \%$ \\
$\mathbf{F . 5 0}$ & $35.7 \%$ & $0.78 \%$ & $45.41 \%$ & $18.08 \%$ & $0.03 \%$ \\
$\mathbf{F . 5 5}$ & $27.97 \%$ & $0.7 \%$ & $53.49 \%$ & $17.82 \%$ & $0.02 \%$ \\
$\mathbf{F . 6 0}$ & $20.92 \%$ & $0.6 \%$ & $61.67 \%$ & $16.79 \%$ & $0.02 \%$ \\
$\mathbf{F . 6 5}$ & $14.41 \%$ & $0.52 \%$ & $69.76 \%$ & $15.3 \%$ & $0.01 \%$ \\
$\mathbf{F . 7 0}$ & $9.04 \%$ & $0.4 \%$ & $77.45 \%$ & $13.1 \%$ & $0.01 \%$ \\
$\mathbf{F . 7 5}^{\circ}$ & $4.7 \%$ & $0.31 \%$ & $84.39 \%$ & $10.59 \%$ & $0.01 \%$ \\
$\mathbf{F . 8 0}$ & $1.72 \%$ & $0.17 \%$ & $90.28 \%$ & $7.82 \%$ & $0.01 \%$ \\
\hline \hline
\end{tabular}

where $\overline{\mathcal{B}}_{f}$ is the instantaneous cluster mass affected by friction work within the transverse volume.

For this analysis, a value of $\delta x=5 \mu \mathrm{m}$ is chosen, and attention is focused only on cases $\mathbf{F . 0} \mathbf{0}^{\circ}, \mathbf{F} . \mathbf{3 0 ^ { \circ }}$ and $\mathbf{F} . \mathbf{6 0}^{\circ}$ as the plastic work predictions are similar to those for the corresponding frictionless cases. Predictions are shown in Fig. 5.5 for $\bar{w}_{p}$ and $\bar{w}_{f}$. For each case, the specific plastic work increases rapidly following initial impact in the vicinity of the wall. Plastic work is concentrated near the wall over the region $0 \leq x \leq 30 \mu \mathrm{m}$ for early time, but gradually diffuses away from the wall with increasing time due to stress transmission between particles. Frictional work is more highly localized along the wall surface due to particle-wall friction, but it also diffuses outward with time due to inter-particle friction. For normal impact, predictions indicate that particles in contact with the wall initially stick resulting in little frictional dissipation; in fact, inter-particle friction during this early time is comparable to particle-wall friction. Subsequent inter-particle contact induces gross slip along the wall inducing a sharp rise in frictional dissipation. For larger impact angles, complex slip-stick-slip reversal occurs leading to an almost instantaneous 
$\left(\mathbf{F} .0^{\circ}\right)$
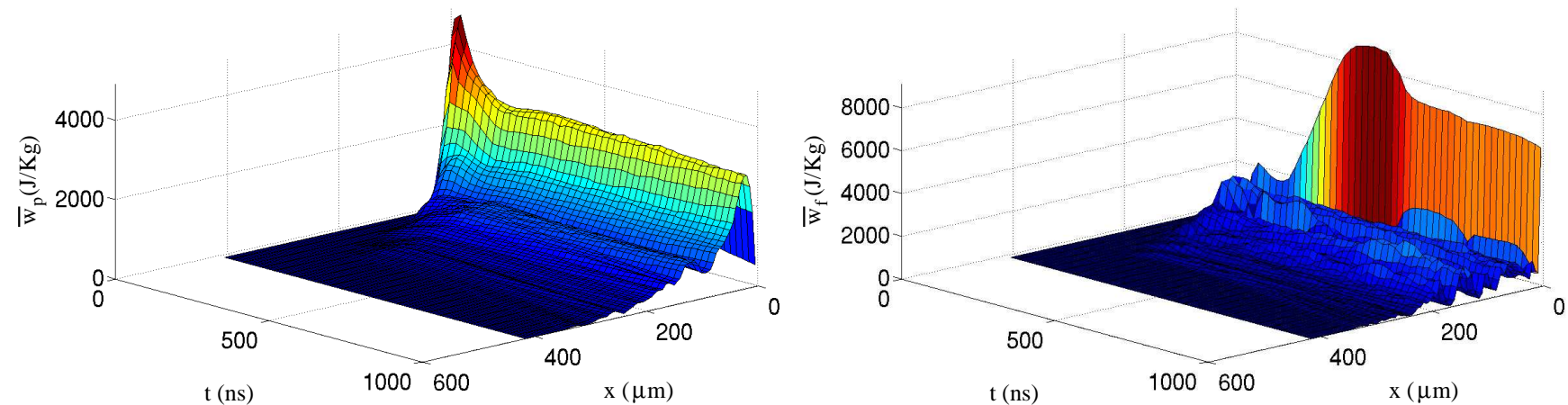

$\left(\mathbf{F} .30^{\circ}\right)$
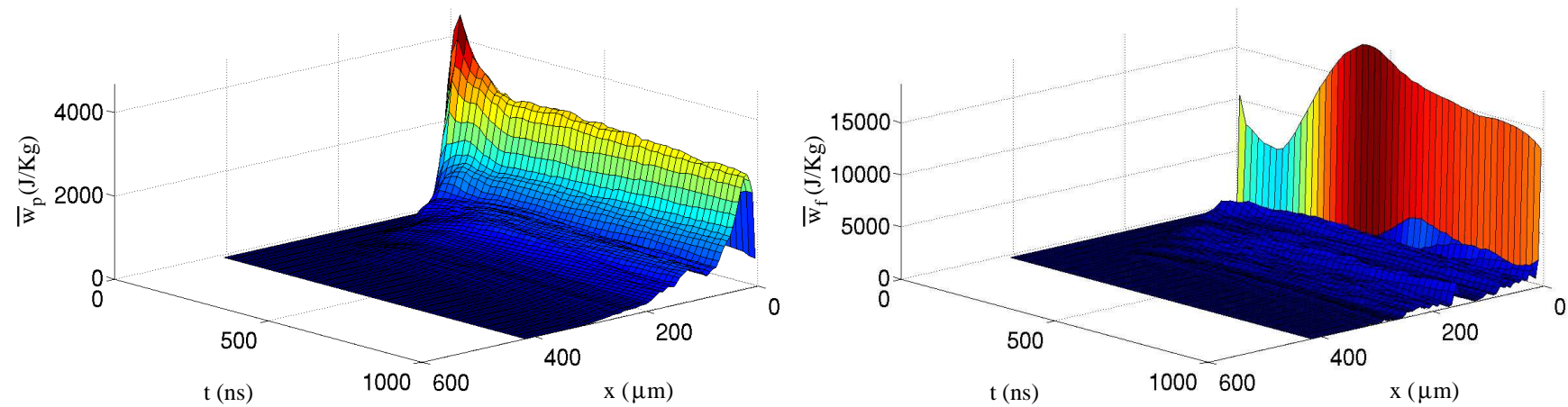

$\left(\mathbf{F} .60^{\circ}\right)$

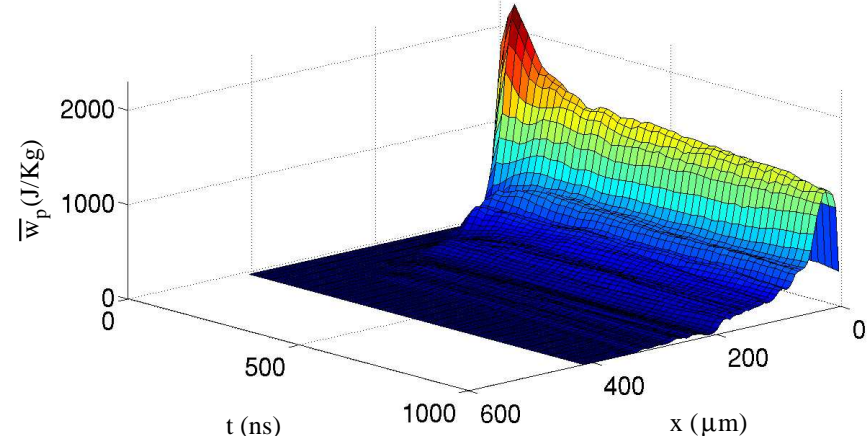

(a)

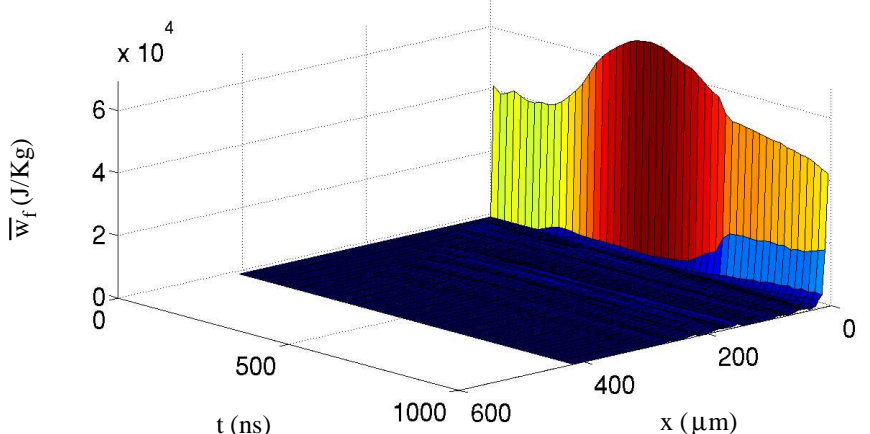

(b)

Figure 5.5: Predictions for the temporal and spatial variation in average specific (a) plastic work and $(b)$ friction work. Here, $x$ is normal distance from the wall.

rise in frictional dissipation. Spatial undulations in plastic and friction work are predicted normal to the wall due successive impact between particles, where the frequency of these undulations is largely dependent on initial particle size and cluster configuration. The mass specific plastic work near the wall is approximately two times larger for case $\mathbf{F} . \mathbf{0}^{\circ}$ than for case $\mathbf{F} . \mathbf{6 0}{ }^{\circ}$, whereas the 
mass specific friction work at the wall is approximately ten times larger for case $\mathbf{F} . \mathbf{6 0}{ }^{\circ}$ than for case $\mathbf{F} . \mathbf{0}^{\circ}$. Particle-wall friction is clearly seen to be more important than inter-particle friction for these simulations, particularly for oblique impact.

\subsubsection{Cluster Temperature}

Predictions for the evolution of temperature within the cluster are now given to highlight the relative importance of plastic and friction work as local heating mechanisms. Here, specific emphasis is placed on characterizing the fraction of mass that is locally heated to elevated temperature as it may have a significant effect on the ignition and subsequent combustion of energetic clusters. Because compression work is small compared to dissipated work for the impact conditions imposed by this study $(<1 \%)$, it is ignored for this analysis.

Temperature fields within individual particles at $t=300 \mathrm{~ns}$ are shown in Fig. 5.6 for the cases $\mathbf{P . 0 ^ { \circ }}$ and $\mathbf{F} \cdot \mathbf{0}^{\circ}$. A complex spatial structure is predicted for each case due to the combined effects of non-homogeneous deformation wave propagation within particles and stress bridging through the cluster by inter-particle contact. Though the spatial fields are dependent on the initial cluster configuration, these predictions are representative of other close-packed configurations in that most potential and dissipated energy is concentrated in particles adjacent to the wall. It is clear from Fig. 5.6 that, for all frictionless cases, the peak temperatures within particles are predicted in the proximity of the wall due to plastic work. Predictions for the corresponding frictional cases indicate that peak temperatures induced by friction work are nearly an order of magnitude larger than those induced by plastic work, and that the frictionally induced thermal fields are much more highly localized near the locations of inter-particle and particle-wall contact. Consequently, the frictionally induced temperature rises are difficult to observe in the figure. Nonetheless, two subtle observations are noteworthy concerning the role of friction on cluster mechanics. First, careful examination and comparison of the frictionless and frictional cases indicates that friction results in the formation of better defined spatially longitudinal energy bands through the cluster. Similar observations have been reported by others for the quasi-static compaction of granular materials 
with friction [137]. Second, friction slightly increases the bulk deformation wave propagation speed within the cluster by effectively making it more "rigid-like." While such a increase in bulk propagation speed may be inconsequential for small particle clusters, it may have meaningful consequences for significantly larger, close-packed particle ensembles.
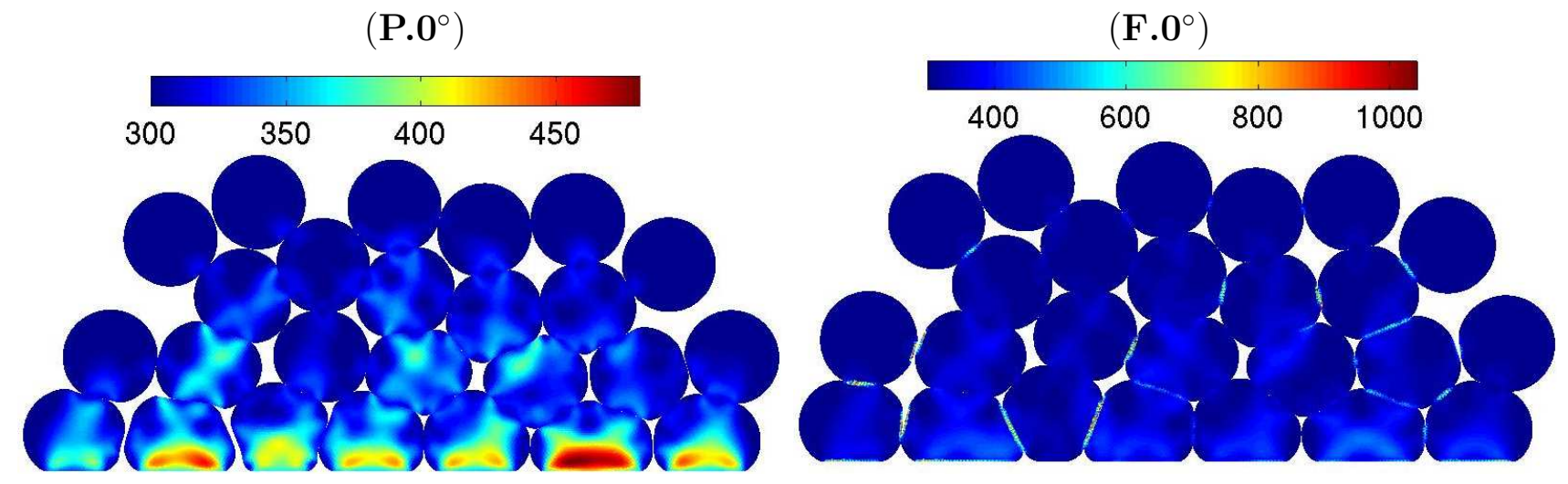

Figure 5.6: Predicted temperature contours within particles at $t=300$ ns. All values shown in the colorbars are in Kelvin.

These predictions indicate that plastic and friction work result in significantly different particle temperature fields. Because plastic work is a volumetric phenomenon, whereas friction work is a surface phenomenon, substantially more mass is affected by plasticity than by friction. For example, nearly $90 \%$ of the cluster mass experiences plastic work for normal impact (case $\mathbf{P . 0 ^ { \circ }}$ ), which reduces to $55 \%$ for highly oblique impact (case $\mathbf{P . 8 0}{ }^{\circ}$ ), whereas approximately $3.5 \%$ of the cluster mass experiences friction work for normal impact (case $\mathbf{F} .0^{\circ}$ ), which reduces to $1.6 \%$ for oblique impact (case $\mathbf{F} \cdot \mathbf{8 0}^{\circ}$ ). However, frictionally induced temperature rises greatly exceed plastically induced temperature rises, as indicated in Fig. 5.6.

To compute the cluster hot-spot mass fraction distribution, the temperature rise axis is partitioned into a finite number of equally spaced intervals, and the fraction of cluster mass having temperature within each interval at a fixed time is determined. In this study, a temperature interval of $\delta T=5 K$ was used for all cases. Figure 5.7 summarizes the predicted hot-spot mass fraction distribution histories for cases $\mathbf{P . 0}{ }^{\circ}, \mathbf{P . 3 0}, \mathbf{P . 6 0}{ }^{\circ}, \mathbf{F . 0}, \mathbf{F . 3 0}{ }^{\circ}$ and $\mathbf{F . 6 0}{ }^{\circ}$. In these plots, the vertical and horizontal axes represent temperature rise and time, respectively; values shown in the colorbar represent the logarithm of mass fraction. The predictions indicate that approximately 
$\left(\mathbf{P} .0^{\circ}\right)$

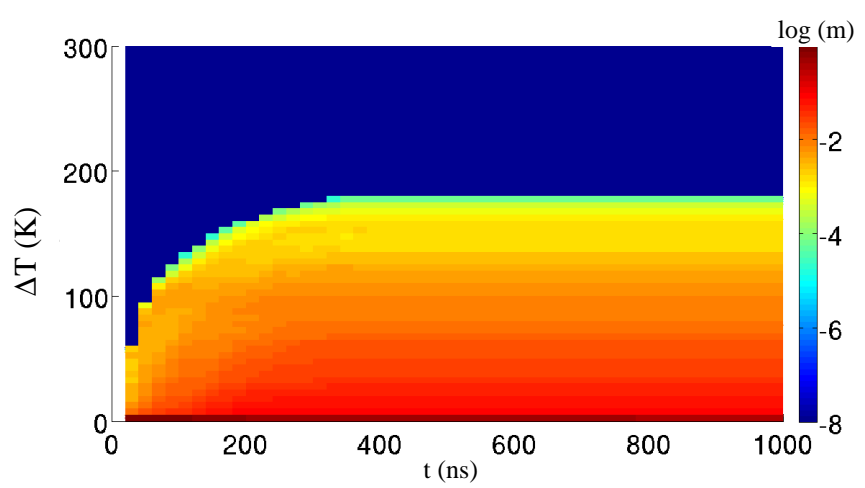

$\left(\mathbf{P} .30^{\circ}\right)$

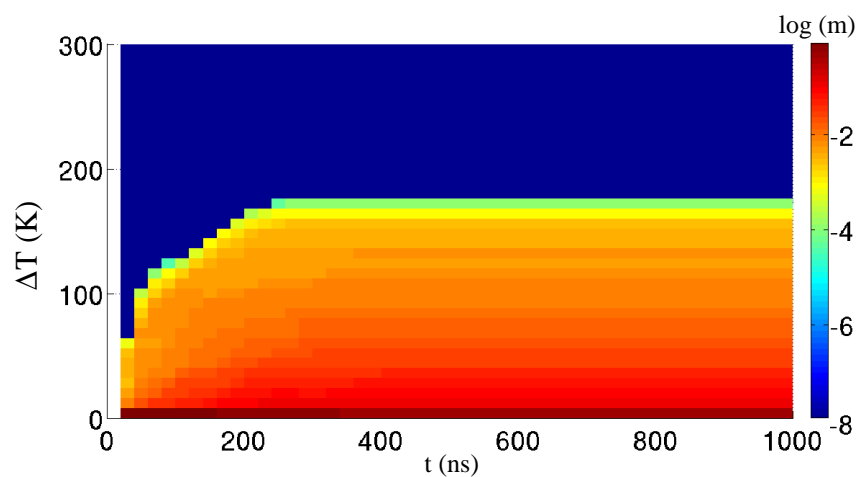

$\left(\mathbf{P . 6 0} 0^{\circ}\right.$

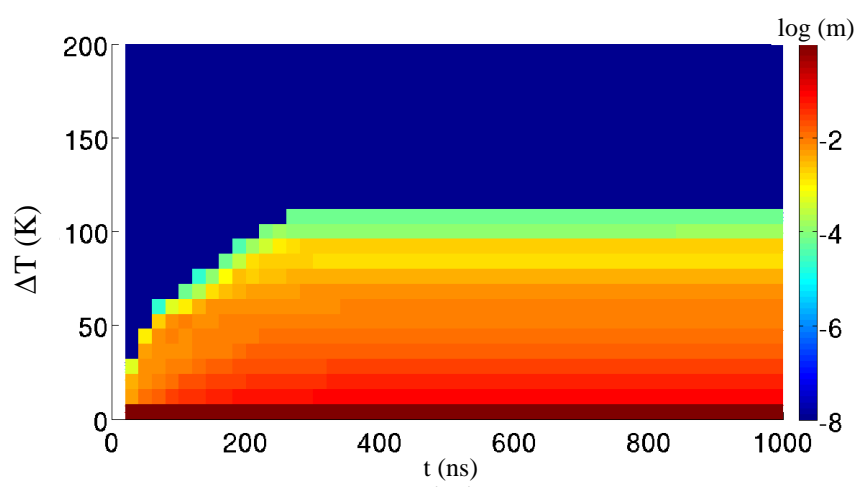

$\left(\mathbf{F} .0^{\circ}\right)$

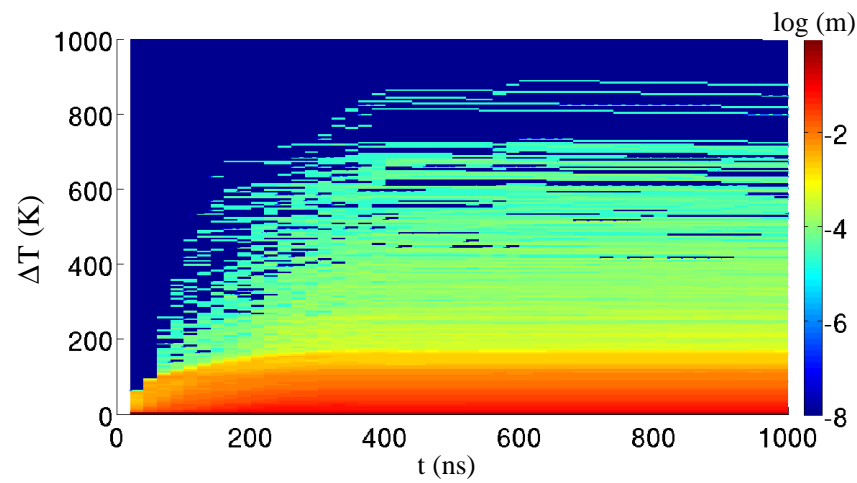

$\left(\mathbf{F} .30^{\circ}\right)$

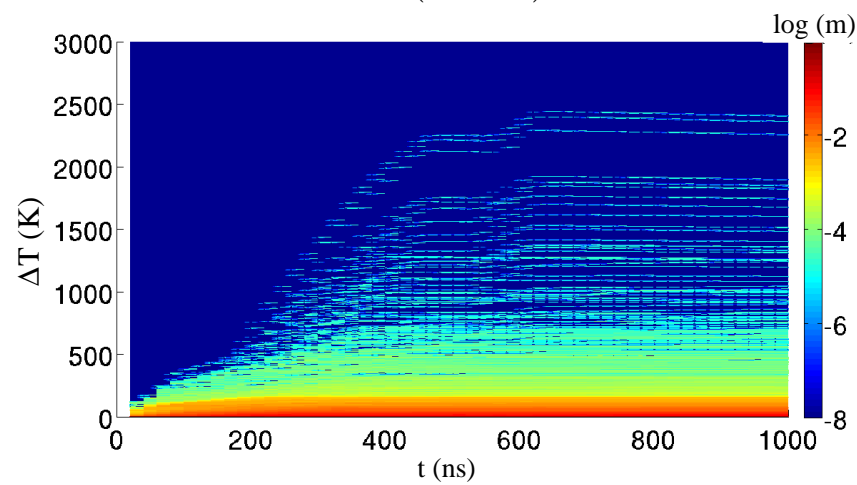

$\left(\mathbf{F} .60^{\circ}\right)$

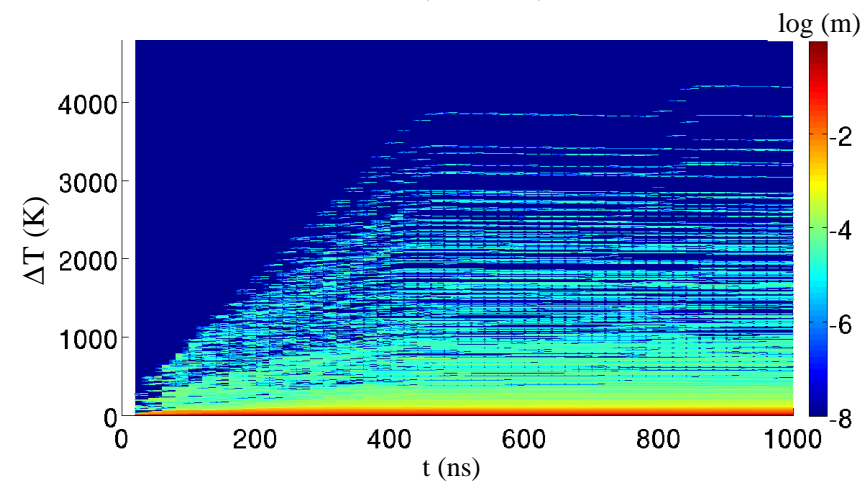

Figure 5.7: Predictions for distribution of hot-spot mass fractions.

$98 \%$ of the cluster mass experiences temperature rises within the range of $0 \leq \Delta T \leq 200 \mathrm{~K}$ for case F. $0^{\circ}$, with most mass only modestly increasing in temperature by $\Delta T \leq 100 \mathrm{~K}$. Much of this bulk mass heating is the result of plastic work. Because these temperature rises are only marginally close to that required for ignition of energetic solids $\left(\Delta T_{i g} \approx 300 \mathrm{~K}[95]\right)$, it is questionable whether plastic heating is a viable ignition mechanism for low impact speeds. Though the cluster mass 
affected by friction work is significantly smaller than that affected by plastic work, frictionally induced local temperature rises far exceed the ignition temperature. For example, approximately $2 \%$ of the cluster mass attains a temperature rise of $\Delta T \geq 200 \mathrm{~K}$ for case $\mathbf{F} . \mathbf{0}^{\circ}$, with a much smaller percentage (.01\%) experiencing temperature rises in excess of $500 \mathrm{~K}$. For case $\mathbf{F} . \mathbf{6 0}^{\circ}$, approximately $1.4 \%$ of the cluster mass experiences $\Delta T \geq 200 \mathrm{~K}$, with less than $.01 \%$ of the cluster mass experiencing $\Delta T>1000 \mathrm{~K}$. In this case, all plastically heated mass has $\Delta T \leq 130 \mathrm{~K}$.

Other observations are noteworthy concerning the hot-spot predictions. First, for the frictionless cases, the maximum plastically heated temperature rise within the cluster monotonically decreases from approximately $180 \mathrm{~K}$ for case $\mathbf{P . 0 ^ { \circ }}$ to approximately $40 \mathrm{~K}$ for case $\mathbf{P} . \mathbf{8 0}^{\circ}$, whereas the maximum total temperature rise within the cluster for the frictional cases increases from approximately $900 \mathrm{~K}$ for case $\mathbf{F} .0^{\circ}$ to $4400 \mathrm{~K}$ for case $\mathbf{F} . \mathbf{8 0}^{\circ}$. Second, almost the entire temperature rise induced by plastic work occurs shortly following initial impact $(<300 \mathrm{~ns})$, beyond which friction work continues to heat due to gross sliding along the wall and between particles. Third, for the short impact times simulated here, effects of thermal conduction are not computationally resolved. As such, mass locally heated to elevated temperature remains at that temperature for the duration of the simulation. These predictions collectively indicate that frictionally induced temperature rises may be very high for a small fraction of the cluster mass. It is plausible that this high temperature mass may play a more important role in combustion initiation of energetic clusters than plastic heating, particularly for weak impact, provided that they possess enough thermal inertia.

\subsection{Sensitivity to Initial Cluster Configuration}

Predictions for configurations 2-4 illustrated in Fig. 5.2 (their properties are listed in Table 5.1) are now briefly summarized to demonstrate the sensitivity of the predictions to variations in initial close-packed particle arrangement for elastic-viscoplastic-frictional impact. The initial close-packed cluster for each configuration consists of 25 well-resolved particles, where the initial particle radii range between $20-70 \mu \mathrm{m}$ for configuration $2,20-80 \mu \mathrm{m}$ for configuration 3 , and 


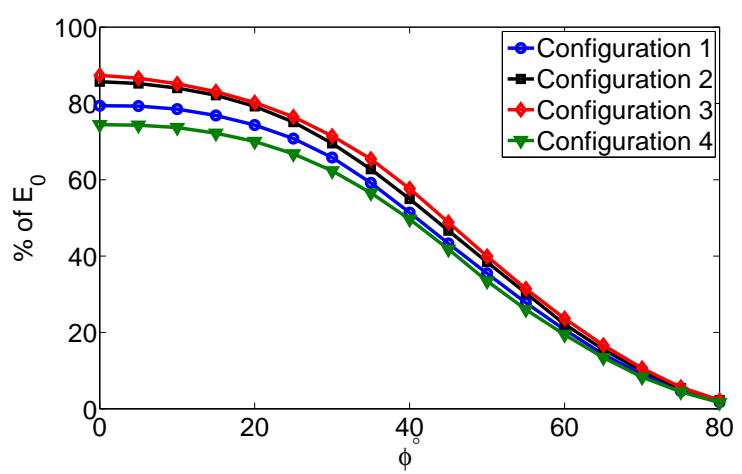

(a)

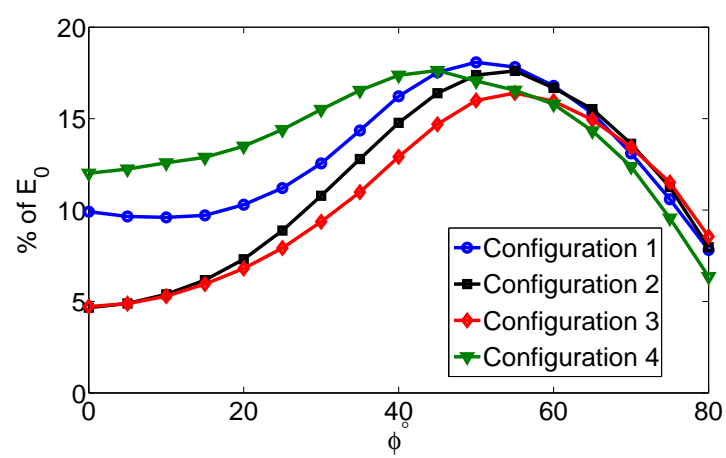

(b)

Figure 5.8: Predicted variation in (a) plastic work (b) friction work with impact angle for all the configurations.

20-90 $\mu \mathrm{m}$ for configuration 4 . Their initial speed is $300 \mathrm{~m} / \mathrm{s}$. The predicted variation in final plastic work and friction work, expressed as a percentage of the initial cluster kinetic energy $E_{o}$, with impact angle is shown in Fig. 5.8 for all configurations. The predictions for plastic work are similar, with the largest disparity occurring for the normal impact case; as such, plastic work is largely insensitive to the initial cluster configuration. For normal impact, the final plastic work for configurations $2-4$ is $85.7 \%, 87.37 \%$ and $74.45 \%$ of their respective $E_{o}$ values. However, the final friction work is sensitive to the initial configuration, with the normal impact cases again exhibiting greatest sensitivity. The final friction work for configurations $2-4$ is $4.66 \%, 4.72 \%$ and $12.01 \%$ of their respective $E_{o}$ values. The maximum friction work occurs for $\phi_{c}^{\circ}=50^{\circ}$ for configurations 1-3, whereas the critical angle for configuration 4 is $\phi_{c}^{\circ}=40^{\circ}$.

The predicted temperature fields for both frictionless and frictional, normal impact cases are shown in Fig. 5.9 for configurations 2-4. The spatial fields are qualitatively similar to that for configuration 1, but differ quantitatively. For frictionless impact, maximum temperature rises of 330,200 and $330 \mathrm{~K}$ are induced for configurations 2-4, respectively, whereas for frictional impact, maximum temperature rises of 800,800 and $1600 \mathrm{~K}$ are predicted. Generally, the net plastic and friction work resulting from cluster impact will depend on its particle size distribution (characterized by $\bar{R}$ and $\widetilde{R}$ ). From this study, it is clear that clusters having non-uniform particle sizes induce greater heating by plastic work. For such cases, maximum temperatures are predicted 
Configuration 2

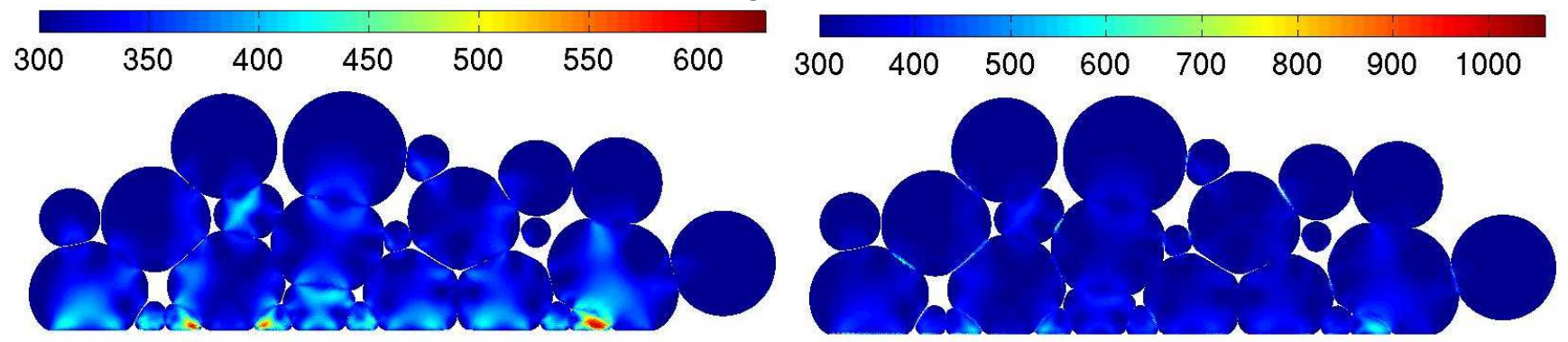

Configuration 3

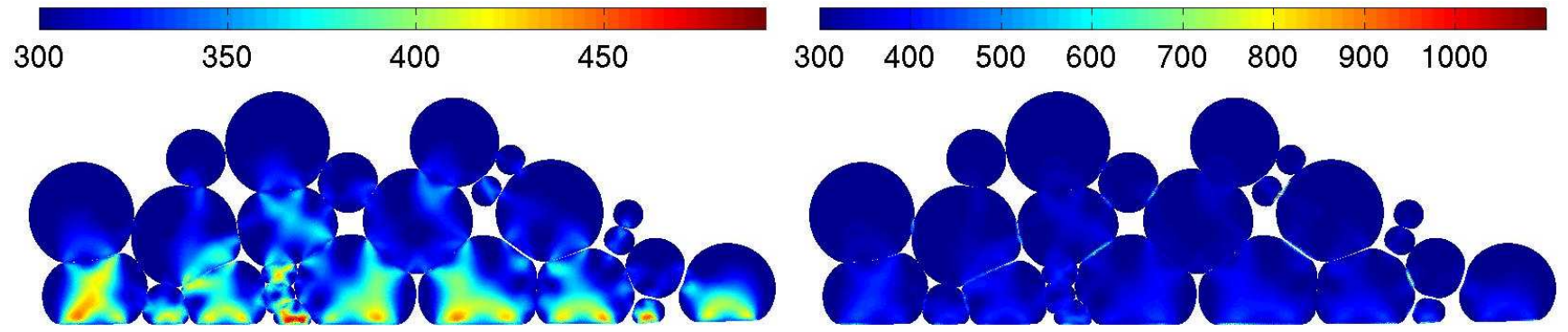

Configuration 4

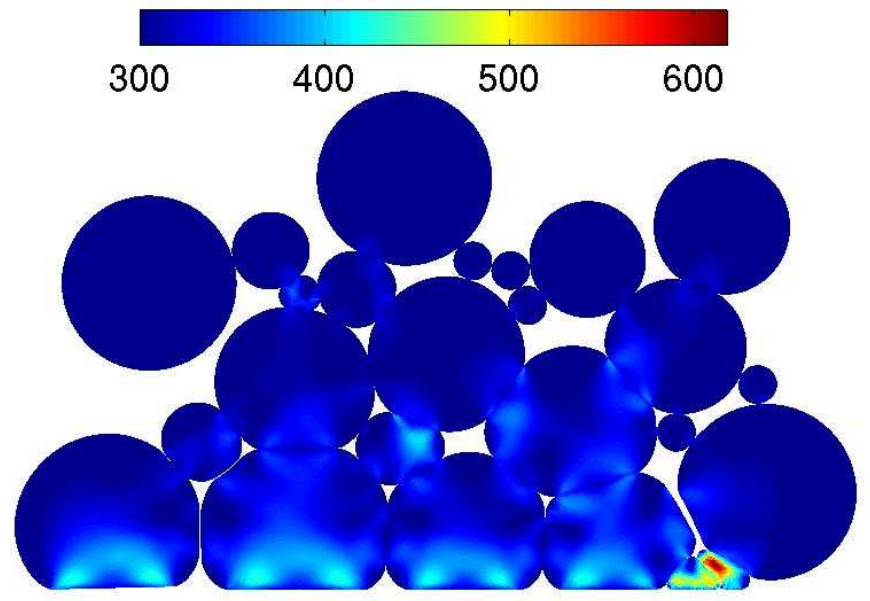

Frictionless

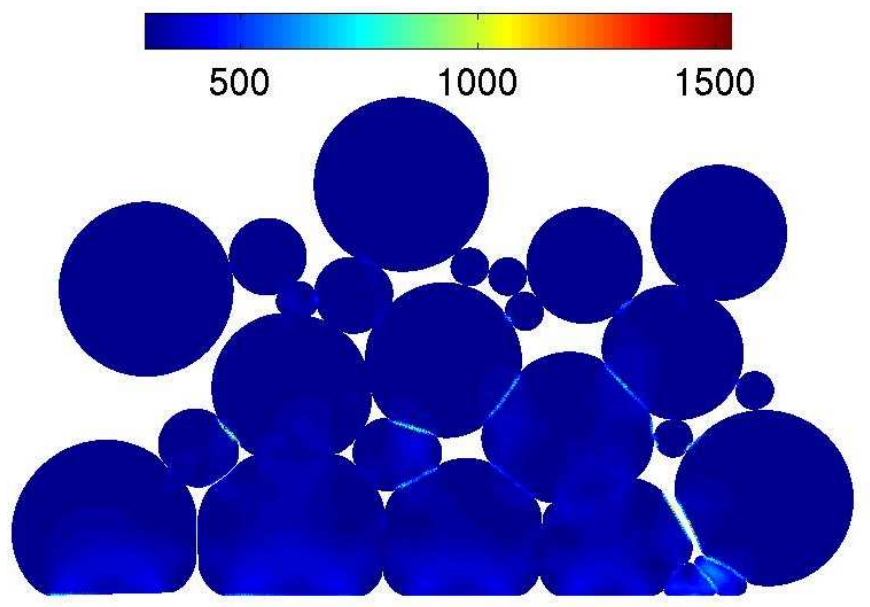

Frictional

Figure 5.9: Predicted temperature contours within particles at $t=300$ ns. All values shown in the colorbars are in Kelvin.

to occur within smaller particles that become pinched between larger particles and the wall. It is also observed that frictional heating is also strongly dependent on particle size. Configuration 4, which has the largest mean particle radius, and the largest particle-wall and particle-particle contact area, experiences the greatest frictionally induced temperature rises. 


\section{Chapter 6}

\section{Uniaxial Deformation Waves}

Predictions are given in this chapter for the simulation of piston supported uniaxial deformation waves in $2 \mathrm{D}$ energetic solids. Techniques used to numerically generate the initial particle ensemble and to post-process simulation data are presented in Section 6.1. Predictions for thermomechanical fields within deformation wave structures and their dependence on piston speed are presented in Section 6.2. Emphasis is placed on characterizing both spatial and temporal variations in local stress and energy states, and the bulk response obtained by spatially averaging these variations over suitable volumes. This discussion is divided into two parts: the mechanical response is first discussed, followed by a discussion of the thermal response.

\subsection{Ensemble Generation and Post-Processing}

To examine the impact response of granular energetic solids, it is important that the particle ensemble reflect actual materials. Ultimately, an accurate meso-scale model for granular explosives should reflect a stochastic geometry that is described by a mixture of energetic particles having a non-uniform size distribution. To this end, several methods such as pseudo-gravity drop methods [15], quantitative stereological methods [128], x-ray computed tomography methods [131], laser scanning confocal microscopy [45] and Monte Carlo/molecular dynamics methods [125] have been used to create realistic initial geometries. In this study, a conventional pseudo-gravity drop method is used to create the initial particle ensemble.

\subsubsection{Ensemble Configuration}

Important things to consider in choosing an initial ensemble configuration are ensemble size, particle size distribution, and initial porosity. The initial particle ensemble used in this study is shown in Fig. 6.1. It consists of 2000, tightly packed, randomly distributed circular particles inside a rectangular domain. Compaction waves are driven by a rigid, constant velocity, planar piston 


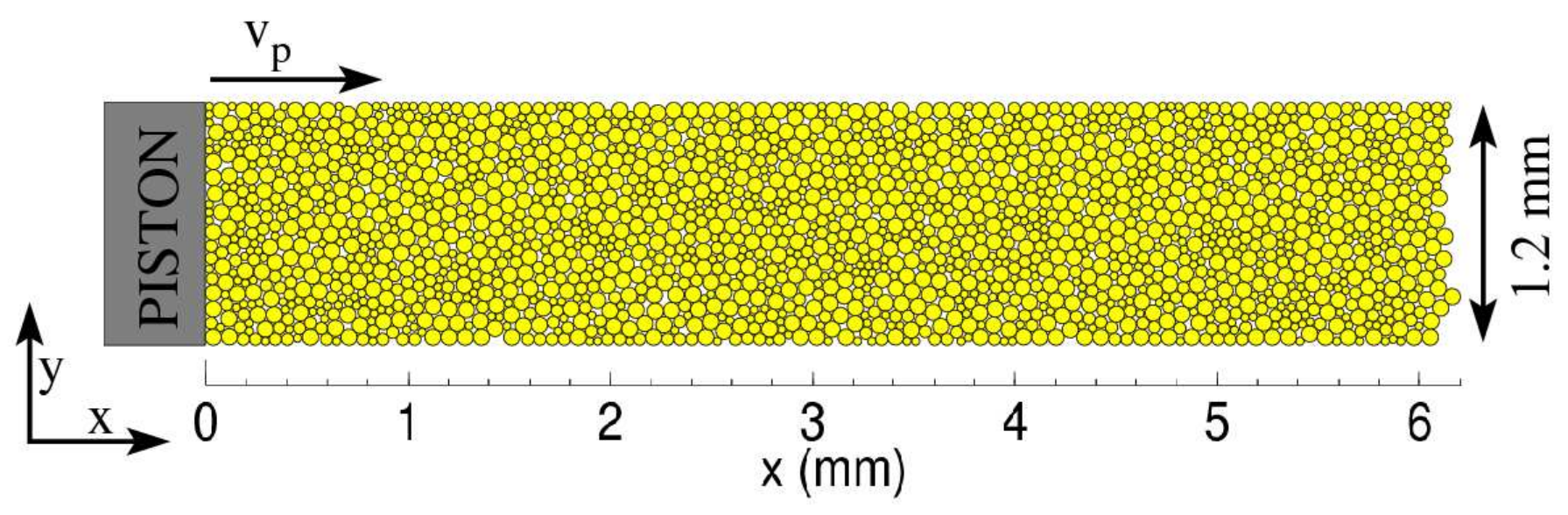

Figure 6.1: Schematic of the initial system configuration used in this study. moving from left to right. The $x$-axis is aligned in the direction of piston and wave propagation, and the $y$-axis is aligned in the transverse direction. The length of the domain is chosen sufficiently large to enable quasi-steady bulk wave structures to evolve. The width of the domain is chosen sufficiently large to accurately describe $2 \mathrm{D}$ effects arising from heterogeneities, and to minimize the effect of transverse boundaries on wave propagation. The ensemble shown in the figure consists of three particle sizes, that is, with an initial radius of 20,30 and $40 \mu \mathrm{m}$ which constitute 33,32 and $35 \%$, by number, of the ensemble, respectively. This configuration corresponds to a conventional fine, non-uniform distribution having a mean particle radius of $30 \mu \mathrm{m}$.

The particle packing algorithm used in this study consists of two steps. In the first step, a preliminary packing arrangement is generated by randomly placing particles within the domain; in so doing, it is required that the centers of newly introduced particles not be located within the volume occupied by existing particles. This preliminary configuration, therefore, has less than $50 \%$ overlap between particle pairs. The domain width, the number of particles, and the particle size distribution are fixed during this process. The second step is based on a molecular dynamics approach whereby forces arising from particle overlap are used to adjust particle positions to eliminate overlap while settling under an applied gravity force. Forces between particles are assumed to be proportional to the amount of overlap. The particles are assumed rigid, and their mass is proportional to their size. Particle displacements are calculated in a manner that reduces 


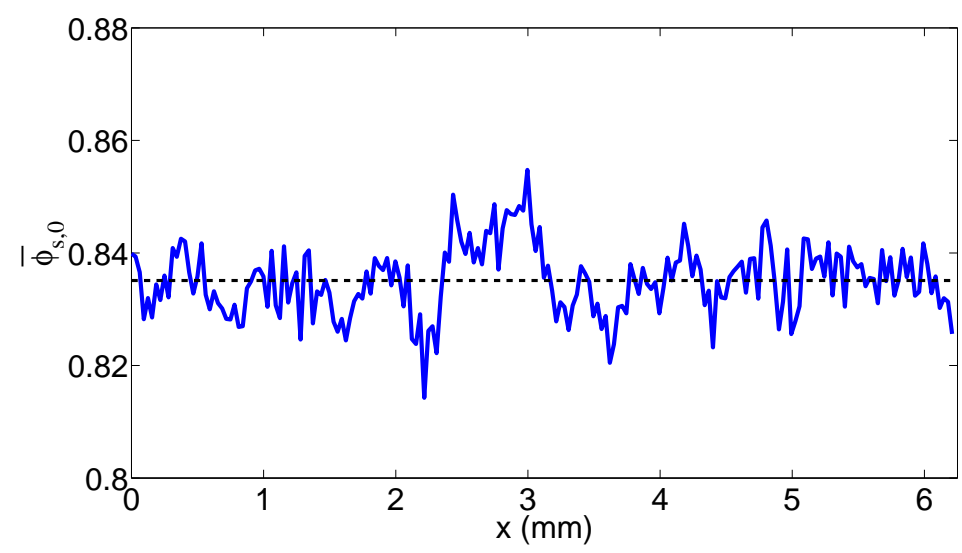

Figure 6.2: Average initial solid volume fraction $\bar{\phi}_{s, 0}$.

the sum of forces acting on each particle. The left, upper, and bottom boundaries of the domain are fixed for this process, whereas the right boundary is open.

Figure 6.2 shows the initial solid volume fraction profile, $\bar{\phi}_{s, 0}(x)$, based on an averaging volume that is 3 particle diameters long $(\approx 200 \mu \mathrm{m})$ and spans the transverse domain width. The numerical strategies used for computing averages are discussed later. This profile is characterized by short wavelength, large amplitude fluctuations due to the heterogeneity. The ensemble has an average solid volume fraction of $83.5 \%$, with global minimum and maximum values of $81.5 \%$ and $84.5 \%$. Though not shown here, the particle setting algorithm also introduces spatial fluctuations in porosity in the $y$-direction. The porosity is maximum on the boundaries and minimum in the interior of the ensemble. This is because the upper and lower boundaries are fixed during settling, which restricts the motion of particles near the boundaries. These observations are indicative of spatially varying meso-structure which can influence deformation wave behavior.

\subsubsection{Parallelization}

In this study particles are discretized using CST finite elements with a uniform element edge length of $\Delta h=3 \mu \mathrm{m}$. The resulting discrete ensemble consists of $\mathcal{N}_{\text {ele }}=1142356$ finite elements and $\mathcal{N}_{n}=633968$ nodes, where each particle contains 280 to 760 finite elements, depending on size. This resolution was chosen based on the available computing power. The numerical algorithm, implemented using a serial FORTRAN 90 code, was executed on a 2.66 GHz Dual Core Xeon 


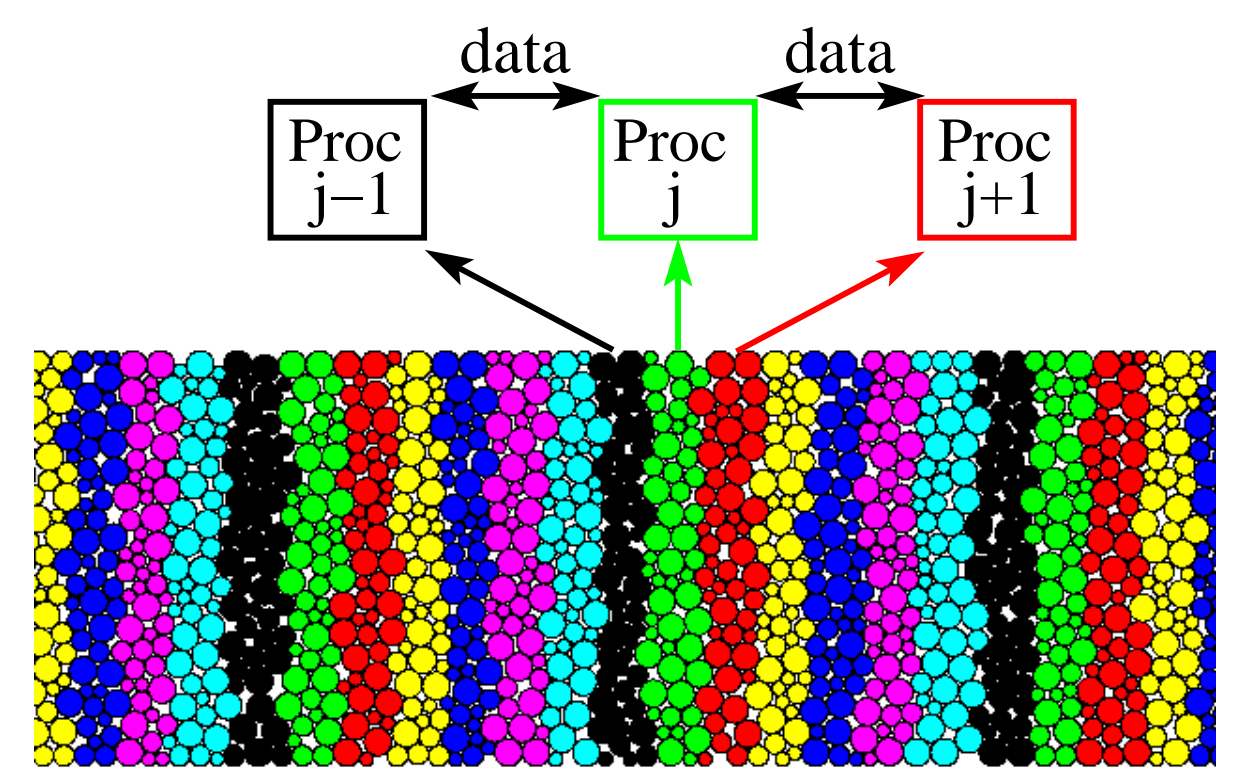

Figure 6.3: Schematic of the initial system configuration used in this study.

64-bit workstation having 4 GB RAM. A typical run time for a single simulation is approximately 10 days. Run times for systems comprising of a larger number of finite elements, for example, an ensemble of 4000 particles with the same particle size distribution or 2000 particles with a wider particle size distribution was approximately 25-30 days. Substantially larger particulate systems may require considerably more computational time. Therefore, to reduce computational time, a parallel version of the FORTRAN 90 code was developed using message passing interface (MPI) and domain decomposition fundamentals. Details of the parallelization procedure are presented next.

The parallel code developed in this study uses multiple processors, each with an individually assigned memory allocation, to perform the same computations as the serial code on different portions of the particle ensemble. Subsequently, the run times for the parallel code are considerably smaller than the serial code. Parallelizing a serial FORTRAN code using domain decomposition schemes generally involves dividing the spatial domain into sub-domains and assigning each subdomain to a specific processor. Each processor then runs the serial version of the FORTRAN code on the sub-domain allocated to it while exchanging information on the boundaries of the sub-domain with other processors. Shown in Fig. 6.3 is the initial particle ensemble along with 


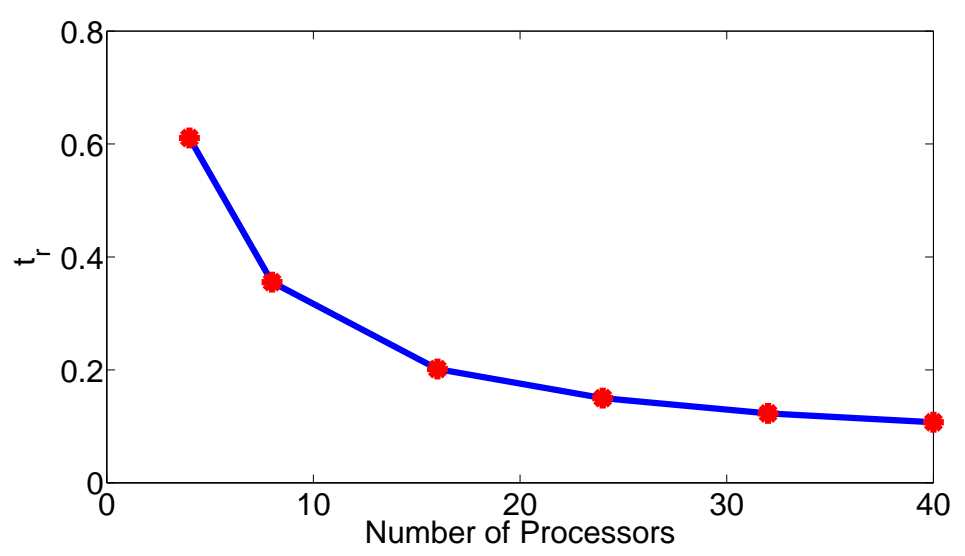

Figure 6.4: Scaling curve for the parallel FORTRAN code.

the sub-domains. Here, the set of particles belonging to different sub-domains are shown using different colours. Generally, the choice of sub-domains is problem specific. For the problem under consideration the simplest choice of the sub-domains are transverse slices of the particle ensemble. After assigning the sub-domains to different processors, each processor proceeds to integrate the solution variables within particles assigned to that processor. However, to model contact between particles belonging to two different processors information needs to be exchanged between the two processors. This is accomplished by using the MPI standard SEND and RECV subroutines. Detailed information on the MPI standard subroutines can be found in reference [54].

To estimate the speed up achieved by by parallelizing the serial code, simulations were performed on the current ensemble using multiple processors. Figure 6.4 plots the variation in the scaling time $t_{r}$ with the number of processors. Here, for a given number of processors $N_{\text {proc }}, t_{r}$ is the ratio of the run time for the parallel code and the serial code. As the figure suggests, the run times for the parallel code are substantially smaller than the serial code. As the number of processors are increased by a small number $\left(N_{\text {proc }}<10\right)$, substantial speed up is achieved due to decreased computation time. In this range the processor communication time is minimal. However, with further increase in $N_{\text {proc }}, t_{r}$ only decreases asymptotically because the speed up achieved due to decreased computation time becomes comparable to the increase in communication time. 


\subsubsection{Postprocessing}

Three types of plots are used in this study to display predicted data. One-dimensional, transverse average plots are used to illustrate bulk axial profiles, color 2D contour plots are used to illustrate thermomechanical fields, and color 2D contour plots are used to display hot-spot mass-fractions. Numerical procedures used to postprocess data into a form suitable for these plots are discussed next.

\section{- Bulk Axial Profiles}

Because bulk, uniaxial deformation waves propagate in the axial $(x)$ direction for this study, it is desirable to characterize wave structure in terms of transverse average, $1 \mathrm{D}$ plots which highlight axial variations in thermomechanical variables. To this end, local fields are averaged with respect to $y$-position to obtain a mean value over $0 \leq x \leq L$, where $L=6.209 \mathrm{~mm}$ is the length of the domain. To compute transverse running averages, a scalar material point function $\psi$ is defined which specifies whether a point in space lies within a particle or a void:

$$
\psi(x, y)= \begin{cases}1 & \text { if } \quad(x, y) \text { is a point inside a particle } \\ 0 & \text { otherwise }\end{cases}
$$

For $2 \mathrm{D}$ particles, the function $\psi$ may be used to locally define the solid volume fraction within an averaging area by

$$
\phi_{s}=\frac{A_{\text {particles }}}{A_{\text {total }}}
$$

where $A_{\text {particles }}$ is the planar area of particles contained within the planar averaging area $A_{\text {total }}$. Figure 6.5 shows a schematic of a typical ensemble with relevant dimensions indicated. For any arbitrary value of $x=\bar{x}$, the averaging area $A_{\text {total }}$ represents a rectangle of length $\delta x$ and width $H$. If $\bar{x}$ is chosen such that $\delta x \leq \bar{x} \leq L-\delta x$, then the averaging area is centered at $\bar{x}$ and is given as $A_{\text {total }}=H \delta x$. If $\bar{x}<\delta x$, which occurs near the piston boundary, then the averaging area is not centered about $\bar{x}$, but is chosen so that its left edge is located at the piston surface, and its right 


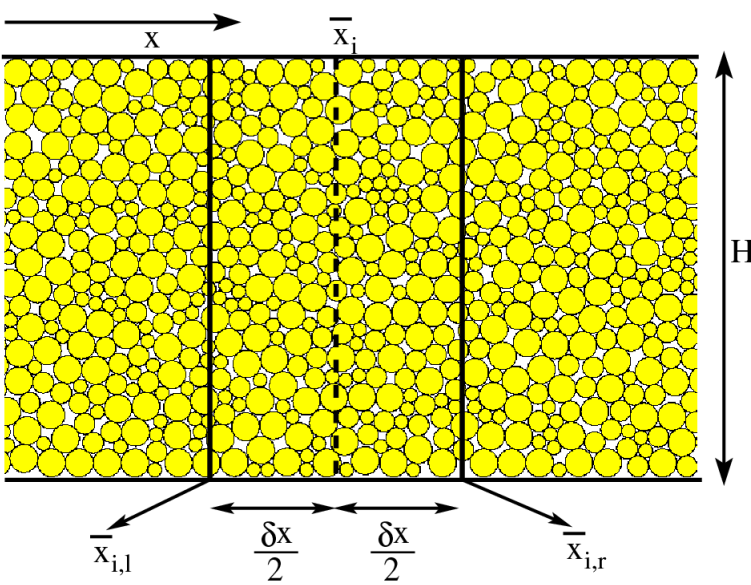

(a)

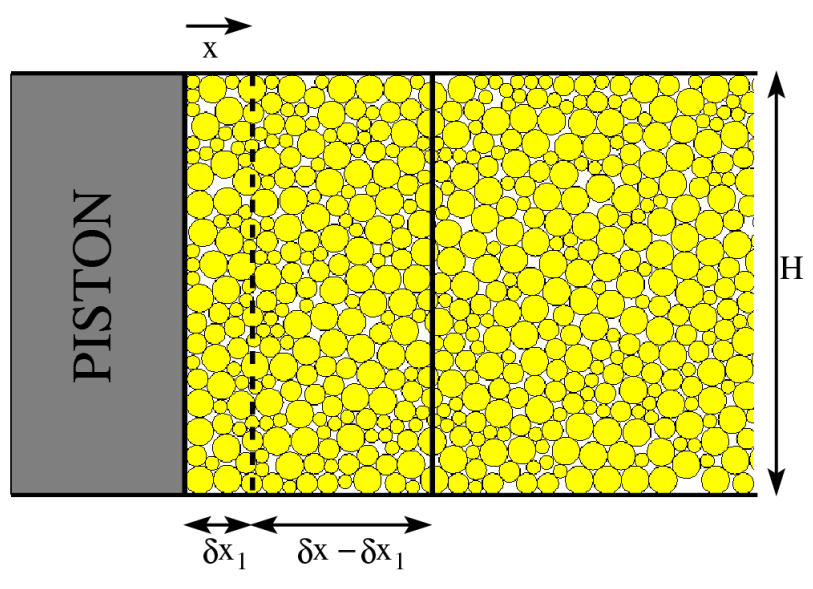

(b)

Figure 6.5: Schematic of the transverse averaging procedure used to obtain 1D profiles. edge is chosen so that $A_{\text {total }}=H \delta x$, as shown in Fig. 6.5(b). An analogous procedure is used near the right (open) axial boundary.

At any point $(x, y)$ within an averaging area, the differential mass per unit length can be defined as $d m=\rho \psi d A$, where $d A=d x d y$ is the differential area. The solid volume fraction may then be expressed by:

$$
\bar{\phi}_{s}(\bar{x})=\frac{\int_{0}^{H} \int_{\bar{x}-\frac{\delta x}{2}}^{\bar{x}+\frac{\delta x}{2}} \psi(x, y) d x d y}{\int_{0}^{H} \int_{\bar{x}-\frac{\delta x}{2}}^{\bar{x}+\frac{\delta x}{2}} d x d y}=\frac{1}{H \delta x} \int_{0}^{H} \int_{\bar{x}-\frac{\delta x}{2}}^{\bar{x}+\frac{\delta x}{2}} \psi(x, y) d x d y
$$

Both mass and area weighted averages are used to display results in this study. Variables which represent mass specific quantities, such as temperature (based on mass specific thermal energy), plastic and friction work (mass specific energy), and velocity (mass specific momentum) are obtained using mass weighted averages. Variables which represent volume specific quantities, such as stress (volume specific energy) are obtained using area weighted averages. For any arbitrary variable $\Phi$, its mass and area weighted transverse averages at $\bar{x}$ are given by

$$
\bar{\Phi}_{m}(\bar{x})=\frac{\int_{0}^{H} \int_{\bar{x}-\frac{\delta x}{2}}^{\bar{x}+\frac{\delta x}{2}} \Phi \rho \psi d x d y}{\int_{0}^{H} \int_{\bar{x}-\frac{\delta x}{2}}^{\bar{x}+\frac{\delta x}{2}} \rho \psi d x d y}, \quad \bar{\Phi}_{a}(\bar{x})=\frac{\int_{0}^{H} \int_{\bar{x}-\frac{\delta x}{2}}^{\bar{x}+\frac{\delta x}{2}} \Phi \psi d x d y}{\int_{0}^{H} \int_{\bar{x}-\frac{\delta x}{2}}^{\bar{x}+\frac{\delta x}{2}} \psi d x d y}
$$


respectively.

Equations (6.3) and (6.4) are continuous transverse averages; these equations must be discretized to estimate approximate averages for the discrete ensemble. To this end, suitable interpolation points must be introduced for the discrete particles. In this study, the interpolation point for each finite element is taken at its centroid. However, because variables such as temperature and velocity are considered nodal quantities, they are determined at finite element nodes. Nodal solution variables are approximated at element centroids using a mass weighted average of their values at the corresponding element nodes. For example, the mass averaged temperature at the centroid of an arbitrary finite element $\Delta^{(j)}$, having global nodal indices $(k, l, m)$, is given by

$$
\hat{T}^{(j)}=\frac{\mathcal{M}(k, k) \mathcal{T}(k)+\mathcal{M}(l, l) \mathcal{T}(l)+\mathcal{M}(m, m) \mathcal{T}(m)}{\mathcal{M}(k, k)+\mathcal{M}(l, l)+\mathcal{M}(m, m)}
$$

where $\mathcal{M}$ and $\mathcal{T}$ are the global mass and temperature matrices, respectively. Similar procedure is adopted to estimate the mass averaged velocity of the finite element on a component by component basis.

Prior to obtaining transverse average profiles for the discrete ensemble, a method to partition the ensemble into averaging areas, and to identify finite elements belonging to each averaging area, must be developed. To this end, a specific number of axial positions $\left(\bar{x}_{1}, \bar{x}_{2} \ldots \bar{x}_{n}\right)$ are first chosen for which transverse averages are to be estimated. Each axial position $\bar{x}_{i}$ is assigned a left and right boundary denoted by $\bar{x}_{i, l}$ and $\bar{x}_{i, r}$, respectively. Each finite element is then placed in its respective averaging area based on the axial coordinate of its centroid. For example, an arbitrary finite element having axial coordinate $x_{c}$ is placed into all averaging areas that satisfy the criteria: $\bar{x}_{i, l} \leq x_{c} \leq \bar{x}_{i, r}$

The smoothness of average profiles depends on both the number of axial positions $n$ and the width of the averaging area $\delta x$, and is more sensitive to the choice of $\delta x$ than $n$. All transverse averages shown in this study were computed using $n=199$ and $\delta x=200 \mu \mathrm{m}$. Minimal variation in the average profiles were predicted for modest variations in $n$ and $\delta_{x}$ about these values. 
Finally, the average solid volume fraction at axial position $\bar{x}_{i}$ is given by

$$
\bar{\phi}_{s}\left(\bar{x}_{i}\right)=\frac{1}{H \delta x} \sum_{j=1}^{\mathcal{N}_{a}(i)} A^{(j)}
$$

where $A^{(j)}$ is the current area of finite element $\Delta^{(j)}$ and $\mathcal{N}_{a}(i)$ is the number of finite elements inside the averaging area associated with axial position $\bar{x}_{i}$. Similarly, discrete forms of the mass and area weighted averages for $\Phi$ are given by

$$
\bar{\Phi}_{m}\left(\bar{x}_{i}\right)=\frac{\sum_{j=1}^{\mathcal{N}_{a}(i)} \hat{\Phi}^{(j)} \rho^{(j)} A^{(j)}}{\sum_{j=1}^{\mathcal{N}_{a}(i)} \rho^{(j)} A^{(j)}}, \quad \bar{\Phi}_{a}\left(\bar{x}_{i}\right)=\frac{\sum_{j=1}^{\mathcal{N}_{a}(i)} \hat{\Phi}^{(j)} A^{(j)}}{\sum_{j=1}^{\mathcal{N}_{a}(i)} A^{(j)}}
$$

respectively, where $\hat{\Phi}^{(j)}$ is the value of the solution variable at the centroid of element $\Delta^{(j)}$, and $\rho^{(j)}$ is its current density. The root mean square (RMS) fluctuations are estimated in the standard way as

$$
\widetilde{\Phi}_{m}\left(\bar{x}_{i}\right)=\sqrt{\frac{1}{\mathcal{N}_{a}(i)} \sum_{j=1}^{\mathcal{N}_{a}(i)}\left(\hat{\Phi}^{(j)} \rho^{(j)} A^{(j)}-\bar{\Phi}_{m}\left(\bar{x}_{i}\right)\right)^{2}}
$$

and

$$
\widetilde{\Phi}_{a}\left(\bar{x}_{i}\right)=\sqrt{\frac{1}{\mathcal{N}_{a}(i)} \sum_{j=1}^{\mathcal{N}_{a}(i)}\left(\hat{\Phi}^{(j)} A^{(j)}-\bar{\Phi}_{a}\left(\bar{x}_{i}\right)\right)^{2}}
$$

\section{- Contour Plots}

Color contour plots are used to display spatial variations in solution variables with the 2D ensemble. Two types of contour plots are presented in this study: finite element contour plots and particle averaged contour plots. In finite element contour plots, each finite element is represented by a color, which depends on the value of the solution variable at its centroid. In particle averaged contour plots, each particle is represented by a color, which depends on the average value of the solution variable for the particle. Variables such as pressure are area averaged, whereas temperature is mass averaged. For example, for an arbitrary particle $\Omega^{(i)}$, which is discretized using $\mathcal{N}_{e}(i)$ finite elements, the discretized form of its mass and area weighted average are given 
by

$$
\breve{\Phi}_{m}\left(\bar{x}_{i}\right)=\frac{\sum_{j=1}^{\mathcal{N}_{e}(i)} \hat{\Phi}^{(j)} \rho^{(j)} A^{(j)}}{\sum_{j=1}^{\mathcal{N}_{e}(i)} \rho^{(j)} A^{(j)}}, \quad \breve{\Phi}_{a}\left(\bar{x}_{i}\right)=\frac{\sum_{j=1}^{\mathcal{N}_{e}(i)} \hat{\Phi}^{(j)} A^{(j)}}{\sum_{j=1}^{\mathcal{N}_{e}(i)} A^{(j)}}
$$

respectively. Finite element contours are useful in demonstrating spatial fluctuations in thermomechanical fields within particles. This is useful in analyzing the number and nature of hot-spots which generally occur on a sub-particle scale. However, regions with large fluctuating thermomechanical fields are expected to be extremely localized within the ensemble. Therefore, from the bulk perspective finite element contours appear uniform and give little information about the spatial variation within the bed. Subsequently, particle averaged contours are used to demonstrate particle scale variations in the ensemble.

\section{- Hot-spot Contours}

A convenient way to illustrate the spatial variation in hot-spot mass-fraction through the deformation wave structure is by $2 \mathrm{D}$ hot-spot contours. To estimate hot-spot mass-fraction for the discrete ensemble, the ensemble is first partitioned into averaging areas, and finite elements are placed within their corresponding averaging areas, by the method illustrated in Fig. 6.5. Furthermore, within each averaging area, finite elements are grouped according to their temperature rise, defined by $\Delta T=\hat{T}-T_{0}$, where $T_{0}$ is the initial ensemble temperature, and $\hat{T}$ is given by Eq. (6.5). This procedure is schematically illustrated in Fig. 6.6. Here, the temperature rise axis is discretized into a prescribed number of equally spaced intervals centered at temperature rise values $\Delta T_{1}, \Delta T_{2}, \ldots, \Delta T_{m}$, such that $\Delta T_{o+1}-\Delta T_{o}=\delta T$, where $\delta T$ is also prescribed. Each interval is bounded by maximum and minimum values given by

$$
\Delta T_{o, t}=\Delta T_{o}+\frac{\delta T}{2}, \quad \Delta T_{o, b}=\Delta T_{o}-\frac{\delta T}{2}
$$

respectively. Each finite element $\Delta^{(j)}$ within an averaging area is placed in the temperature rise interval which satisfies the criterion $\Delta T_{o, b} \leq \Delta T^{(j)} \leq \Delta T_{o, t}$. Finally, the mass-fraction corresponding to each cell $(i, o)$, which is bounded by $\Delta T_{o, t}, \Delta T_{o, b}, \bar{x}_{i, l}$, and $\bar{x}_{i, r}$ on the top, bottom, left, and 


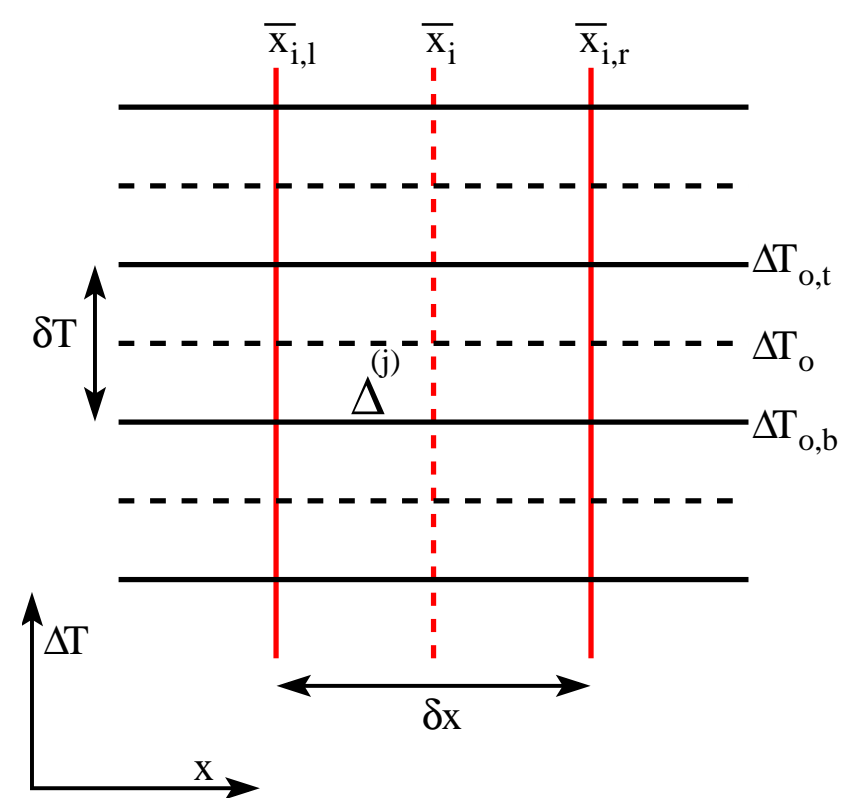

Figure 6.6: Schematic of the discretization procedure used to obtain hot-spot contour plots.

right, respectively, is estimated by

$$
m_{i, o}=\frac{\sum_{j=1}^{\mathcal{N}_{a}(i, o)} \rho^{(j)} A^{(j)}}{\sum_{j=1}^{\mathcal{N}_{a}(i)} \rho^{(j)} A^{(j)}},
$$

where $\mathcal{N}_{a}(i, o)$ is the number of finite elements in cell $(i, o)$. For all the hot-spot contours shown in this study, $n=199, \delta T=5 K$, and $\delta x=200 \mu m$.

\subsection{Predictions}

In this section, predictions are given that illustrate how piston speed $v_{p}$ affects deformation wave structure. To this end, piston speed is varied from $v_{p}=50 \mathrm{~m} / \mathrm{s}$ to $500 \mathrm{~m} / \mathrm{s}$, in $50 \mathrm{~m} / \mathrm{s}$ increments. For each simulation, all material and numerical parameters are fixed at the baseline values listed in Table 4.1. The piston is initially located at $x=0$ for $t=0$. Wave profiles and contour plots given in this chapter correspond to $t=2.75 \mu \mathrm{s}$, unless otherwise stated.

\subsubsection{Mechanical Response}

Though the response of the particle ensemble depends on piston speed $v_{p}$, predictions generally indicate the presence of a two-wave structure within the compaction zone. This structure 


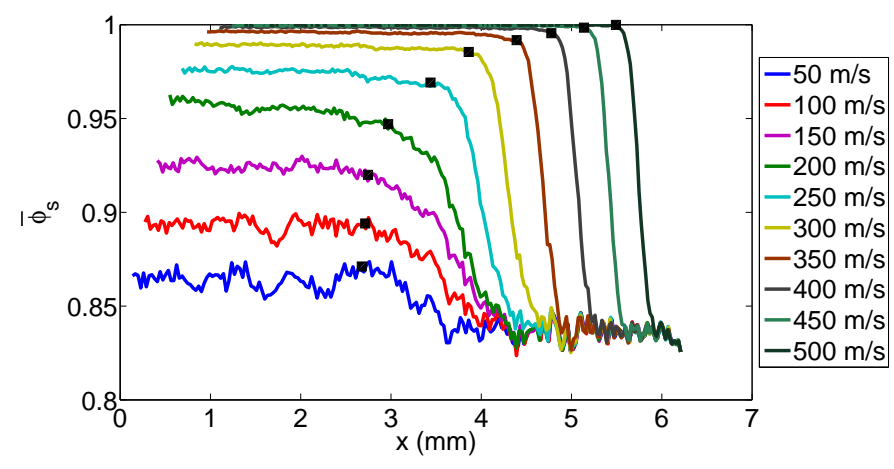

(a)

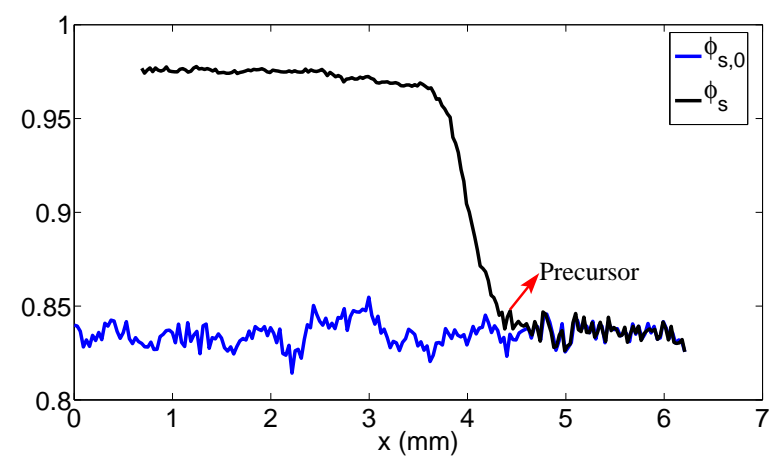

(b)

Figure 6.7: Predictions for the average solid volume fraction $\bar{\phi}_{s}$ at $2.75 \mu \mathrm{s}$ for (a) $v_{p}=50-500 \mathrm{~m} / \mathrm{s}$ (b) $v_{p}=250 \mathrm{~m} / \mathrm{s}$.

consists of a weak, largely elastic precursor followed by a much stronger plastic trailing wave. The precursor is characterized by mild variations in thermomechanical fields, whereas the trailing wave is characterized by more intense changes in these fields. To illustrate the effect of piston speed on compaction dynamics, variations in solid volume fraction $\phi_{s}$ within the compaction zone are first discussed.

\section{- Compaction Dynamics}

All profiles predicted in this study are shown in a fixed, laboratory frame; therefore, the piston boundary location varies with impact speed in the plots. To demonstrate the influence of piston speed on compaction dynamics, predictions are shown for $v_{p}=50,250$, and $500 \mathrm{~m} / \mathrm{s}$, which bound the range of speeds considered in this study. Figure 6.7(a) shows spatial profiles for the average solid volume fraction $\bar{\phi}_{s}$ for all cases. Shown in Fig. 6.7(b) is the corresponding profile for $v_{p}=250 \mathrm{~m} / \mathrm{s}$, along with $\bar{\phi}_{s, 0}$. The predictions indicate that, for all piston speeds, $\bar{\phi}_{s}$ increases from its initial ambient value $\bar{\phi}_{s, 0}$ to a quasi-steady final compacted value $\bar{\phi}_{s, f}$ within the compaction zone; the end of the compaction zones are indicated by black squares in the figure. Details of the compaction zone structure and length are discussed later. However, a few cursory observations are noteworthy. First, $\bar{\phi}_{s, f}$ increases with piston speed; for $v_{p}=50 \mathrm{~m} / \mathrm{s}$, the average solid volume fraction only marginally increases to $\bar{\phi}_{s, f}=0.852$ following compaction, which increases to $\bar{\phi}_{s, f}=$ 0.97 and 1.0 for $v_{p}=250$ and $500 \mathrm{~m} / \mathrm{s}$,respectively. Thus, little compaction occurs for low 
impact speeds, which is mostly attributable to rigid particle rearrangement rather than particle deformation. The particle ensemble is fully compacted (i.e., $\phi_{s, f}=1$ ) for $v_{p} \geq 400 \mathrm{~m} / \mathrm{s}$ due to extensive inelastic particle deformation. Second, the compaction wave is more dispersed at low piston speeds. In this case, it is difficult to identify separate precursor and plastic waves, whereas the split-wave structure is readily apparent for higher piston speeds. For $v_{p}=250 \mathrm{~m} / \mathrm{s}$, the precursor region begins at $x=4.4 \mathrm{~mm}$ and terminates at $x=4.8 \mathrm{~mm}$, as shown in Fig. 6.7(b). Third, the high frequency oscillations present in the initial volume fraction profile remain in the compacted state for $v_{p} \leq 200 \mathrm{~m} / \mathrm{s}$, though the amplitude of the oscillations decreases with piston speed as the meso-structure is consolidated by compaction, resulting in a mostly uniform profile for $v_{p} \geq 350 \mathrm{~m} / \mathrm{s}$.

To further illustrate the effect of piston speed on compaction dynamics, particle configurations near the piston boundary are shown in Fig. 6.8 for $v_{p}=50,250$ and $500 \mathrm{~m} / \mathrm{s}$ at $t=1.5 \mu$ s. Piston displacement is indicated by the shaded rectangular box in the figures, and the positions of the head and tail of the compaction zone are respectively indicated by the solid and dashed lines. The predictions indicate very little particle deformation for $v_{p}=50 \mathrm{~m} / \mathrm{s}$, whereas significantly more compaction occurs for $v_{p}=250 \mathrm{~m} / \mathrm{s}$ due to plastic deformation and particle rearrangement. Close inspection of the predictions reveals that some void space persists within local regions having a larger number of similar sized particles; some of these regions are highlighted by solid circles in Fig. 6.8. In contrast, void space is effectively eliminated in regions having a large number of different size particles, which are indicated by dashed circles in the figure. This observation may be attributed to two factors. First, the initial local porosity for regions having multiple size particles is lower due to higher packing efficiency. Second, because small particles have greater mobility, they are more easily displaced by the wave into void spaces existing between larger particles. This observation is consistent with the experiments of Lowe and Longbottom [85], which indicate that particle morphology and mobility dictate ensemble strength and, thus, $\bar{\phi}_{s, f}$. This observation has two important implications. First, greater particle mobility may result in higher frictional dissipation which can enhance hot-spot formation during compaction. Second, 


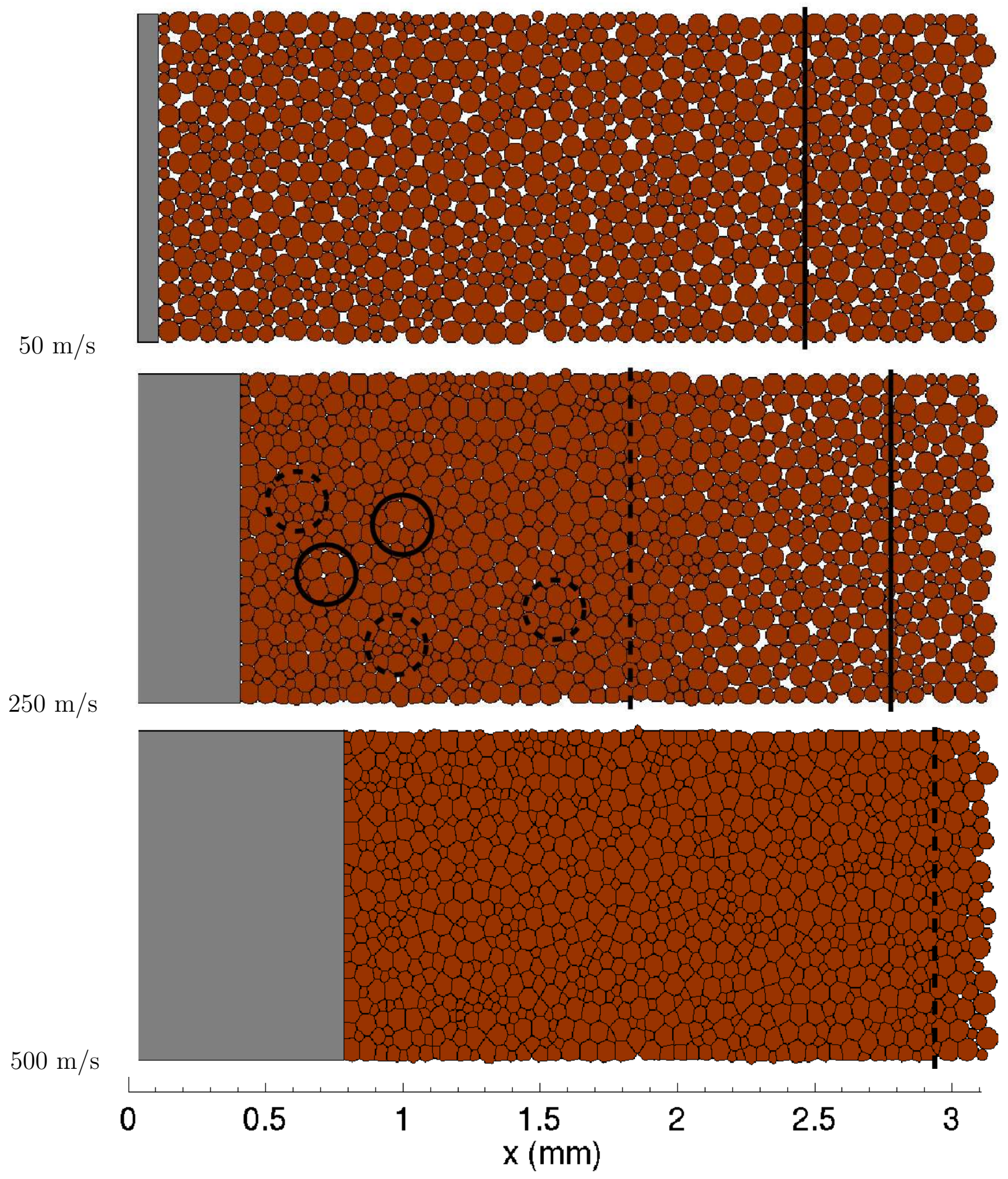

Figure 6.8: Predicted particle positions for $v_{p}=50,250$ and $500 \mathrm{~m} / \mathrm{s}$ at $1.5 \mu \mathrm{s}$. 
compaction of ensembles having a large particle size distribution may result in higher $\phi_{s, f}$ for a given piston speed. Most bulk descriptions of compaction typically model material strength as a function of porosity, where porosity is taken to be independent of particle morphology; this modeling assumption may only be appropriate for high impact speeds (i.e., $v_{p} \geq 400 \mathrm{~m} / \mathrm{s}$ ) which result in complete compaction behind the wave. Therefore, it is plausible that compaction wave behavior is more sensitive to particle morphology at lower piston speeds. Therefore, an additional study is warranted to better characterize how particle morphology affects material strength and compaction wave behavior for mild impact. A detailed discussion on the influence of particle size distribution on the system thermomechanics is presented in the next chapter.

\section{- Velocity Response}

Figure $6.9(\mathrm{a})$ shows profiles of the average axial velocity $\bar{v}_{x}$ for $v_{p}=50$ and $500 \mathrm{~m} / \mathrm{s}$ at time increments $0.25 \mu \mathrm{s}$ following initial impact. These profiles illustrate compaction wave propagation through the particle ensemble. Profiles corresponding to $t=2.75 \mu$ s are highlighted in red in the figure. Though heterogeneities give rise to fluctuations behind the compaction wave, the axial velocity profiles remain largely smooth, even for weak impact. Predictions for $v_{p}=50 \mathrm{~m} / \mathrm{s}$ indicate that $\bar{v}_{x}$ gradually increases from its ambient state through the thick wave structure and matches the piston speed close to the piston surface, as required by the boundary condition. For larger piston speeds, $\bar{v}_{x}$ increases sharply at the wave front and displays largely uniform behavior behind the compaction wave. The compaction wave exhibits a dispersed structure for low piston speeds, and a "shock-like" structure for high piston speeds.

Based on particle motion during the compaction process, the granular bed can be largely divided into three regions; the compaction region, where particles experience the largest accelerations; the compacted region, where particles move at the speed of the piston after the compaction wave has gone through; and the ambient region where particles have not yet been influenced by

the wave. As mentioned, the compaction zone itself is divided into two sub-regions, that is, the precursor and plastic wave region. The wave structure is best illustrated by the RMS fluctuation profiles, computed by Eq. (6.8), and shown in Fig. 6.10(a) for $v_{p}=50$ and $500 \mathrm{~m} / \mathrm{s}$; shown 
$50 \mathrm{~m} / \mathrm{s}$

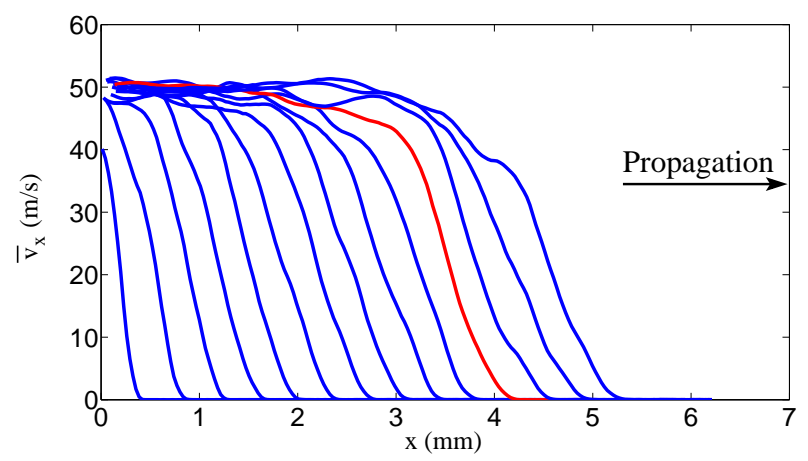

(a)
$500 \mathrm{~m} / \mathrm{s}$

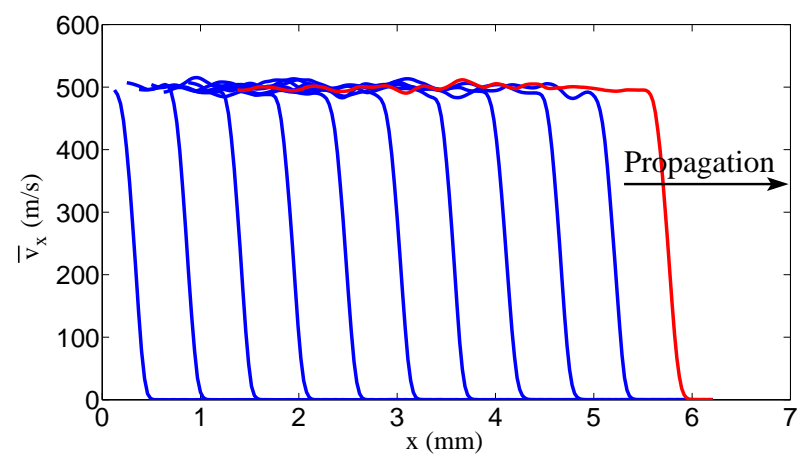

(b)

Figure 6.9: Predictions for the temporal variation of the average axial velocity $\bar{v}_{x}$ for (a) $50 \mathrm{~m} / \mathrm{s}$ and (b) $500 \mathrm{~m} / \mathrm{s}$ case. Profiles are shown in time-steps of $0.25 \mu \mathrm{s}$.

in Fig. 6.10(b) are the corresponding profiles at $t=2.75 \mu \mathrm{s}$, with the ambient and compacted quasi-equilibrium states, and the compaction zones, indicated. Profiles of $\widetilde{v}_{x}$ have a "pulse-like" structure, with the peak occurring within the compaction zone, and the amplitude increasing with $v_{p}$. The fluctuations rapidly decay outside of the compaction zone. Here, the compaction zone length is characterized by the pulse length. This analysis predicts compaction wave lengths of approximately 1.8 and $0.55 \mathrm{~mm}$ for $v_{p}=50$ and $500 \mathrm{~m} / \mathrm{s}$, respectively.

Predictions for the position and length of the compaction zone can also be estimated from the average transverse velocity profiles $\widetilde{v}_{y}$ in a similar manner. These profiles, along with profiles of the RMS fluctuations $\bar{v}_{y}$, are shown in Fig. 6.11 for $v_{p}=50$ and $500 \mathrm{~m} / \mathrm{s}$. Although 2D heterogeneities lead to minor fluctuations in $\bar{v}_{y}$, for all piston speeds, it is only a small fraction of $v_{p}$, and is nearly zero, as expected for uniaxial waves. Profiles of $\widetilde{v}_{y}$ are qualitatively similar to those of $\widetilde{v}_{x}$. Two observations are noteworthy. First, for all cases, $\widetilde{v}_{x}$ is highest close to the piston boundary following impact due to the material and geometry mismatch between the rigid, planar, piston and the initially stationary, circular, deformable particles. For this same reason, the largest inelastic deformation rates $\left(\approx 10^{5}-10^{7} \mathrm{~s}^{-1}\right.$ for $\left.v_{p}=50-500 \mathrm{~m} / \mathrm{s}\right)$ also occur in the immediate vicinity of the piston boundary, as discussed later. This observation is consistent with the micro-particle cluster predictions discussed in the previous chapter, which also indicated that the maximum plastic work within the cluster occurs close to the piston surface immediately following impact. 
$50 \mathrm{~m} / \mathrm{s}$
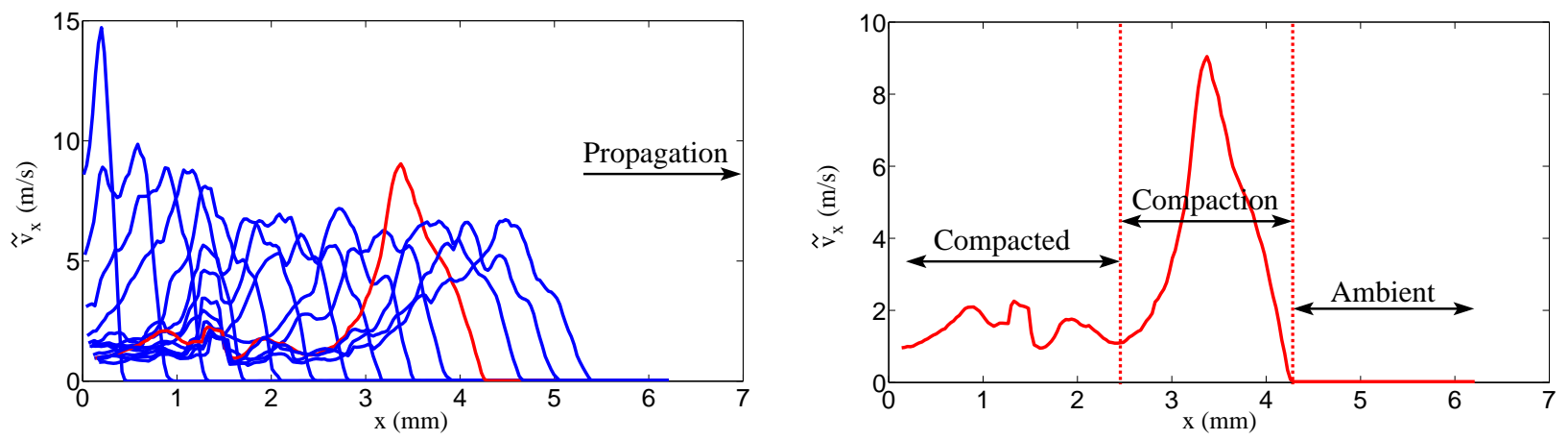

$500 \mathrm{~m} / \mathrm{s}$

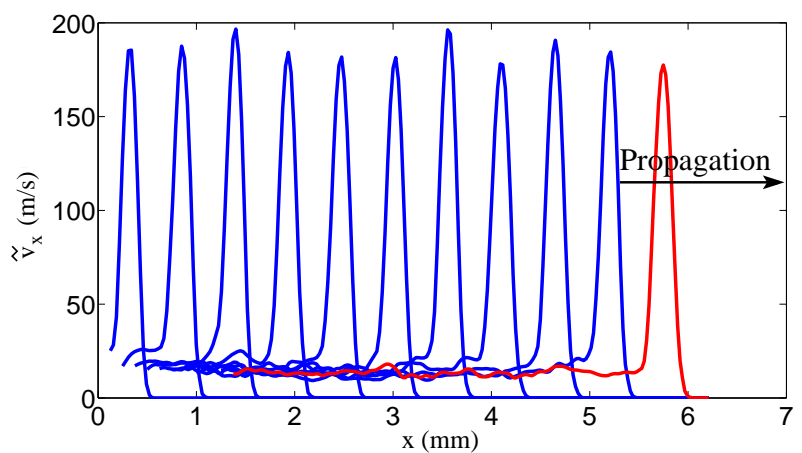

(a)

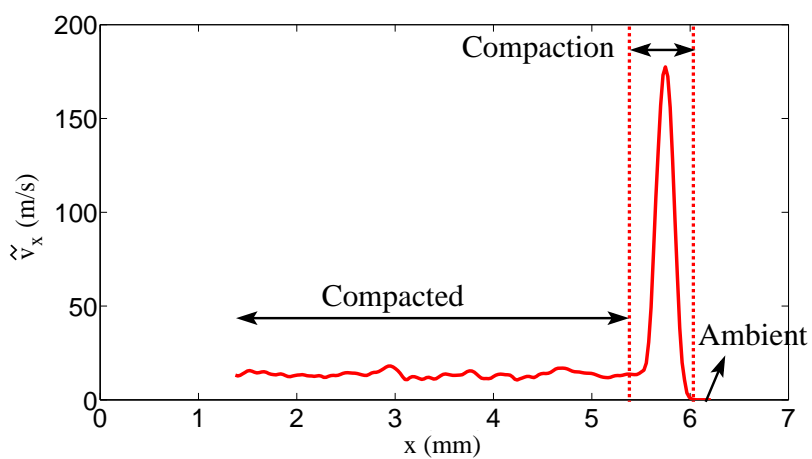

(b)

Figure 6.10: (a) Predictions for the temporal variation of the RMS fluctuation in axial velocity $\widetilde{v}_{x}$ for $v_{p}=50$ and $500 \mathrm{~m} / \mathrm{s}$. Profiles are shown in time-steps of $0.25 \mu \mathrm{s}$. (b) Predictions for the RMS fluctuation in axial velocity $\widetilde{v}_{x}$ at $2.75 \mu \mathrm{s}$ for $v_{p}=50$ and $500 \mathrm{~m} / \mathrm{s}$.

Second, particles within the quasi-equilibrium compacted region subsequently experience only small residual deformations due to internal wave reflections. Because small deformations can induce large stresses within solids, these reflections may cause significant spatial fluctuations in stress fields behind the wave. Importantly, residual particle motion in the compacted region can enhance frictional dissipation that may further increase the intensity of existing hot-spots.

\section{- Stress Response}

Predicted profiles for the average pressure $\bar{p}$ are shown in Fig. 6.12(a) for $v_{p}=50,250$, and $500 \mathrm{~m} / \mathrm{s}$ in time increments of $0.25 \mu \mathrm{s}$. These profiles are a convenient illustration of compaction wave strength and its dependence on piston speed. Profiles corresponding to $t=2.75 \mu s$ are shown in Fig. 6.12(b); the compaction zone lengths are indicated in the figure. These pressure profiles are qualitatively similar to the average axial velocity profiles, with the exception that $\bar{p}$ exhibits more 
$50 \mathrm{~m} / \mathrm{s}$

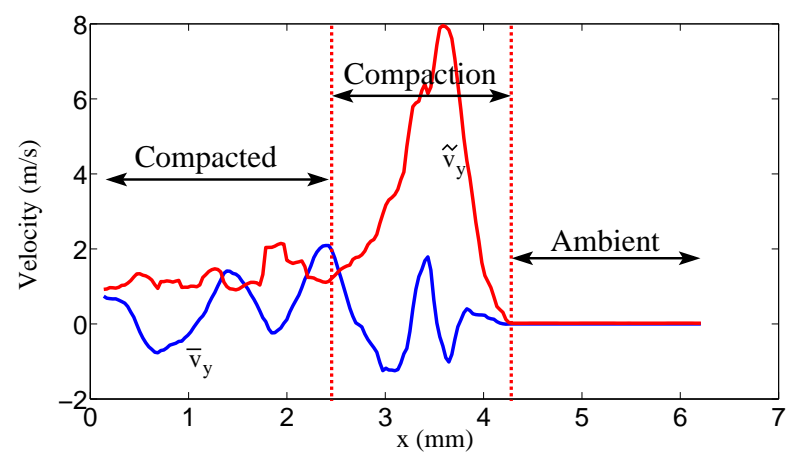

$500 \mathrm{~m} / \mathrm{s}$

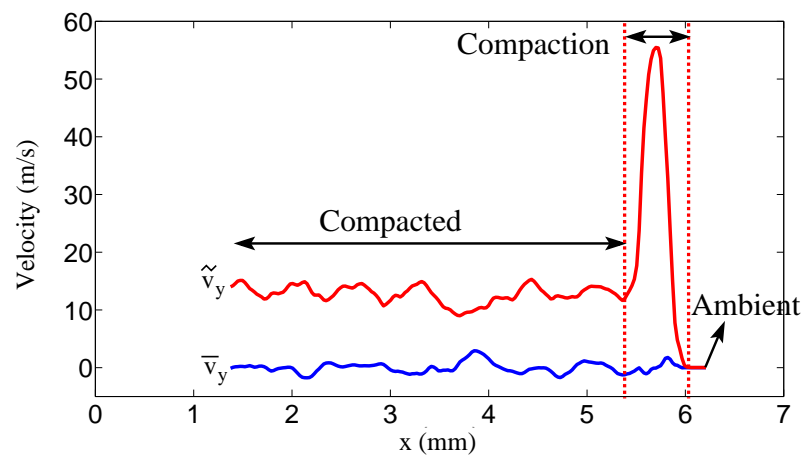

Figure 6.11: Predictions for the RMS fluctuation in transverse velocity $\widetilde{v}_{y}$ at $2.75 \mu \mathrm{s}$ for $v_{p}=50$ and $500 \mathrm{~m} / \mathrm{s}$.

appreciable fluctuations within the compacted region due to wave reflections within and between particles. The predicted pressure increases with $v_{p}$. Behind the compaction wave, $\bar{p} \approx 0.08,0.5$, and 1.6 GPa for $v_{p}=50,250$, and $500 \mathrm{~m} / \mathrm{s}$, respectively. The crush-up pressure $p_{c}$ is defined as the minimum equilibrium pressure for which porosity is eliminated; for $\mathrm{HMX}, p_{c} \approx 0.3 \mathrm{GPa}$ [96]. Though the wave pressure for $v_{p}=250 \mathrm{~m} / \mathrm{s}$ exceeds this value of $p_{c}$, corresponding predictions for $\bar{\phi}_{s}$ indicate that a small amount of void space remains in the compacted region. This small amount of porosity remains because, for reasonably low piston speeds, stress states within the particle ensemble are highly non-uniform due to heterogeneities occurring within the meso-structure. This assertion is best illustrated by particle averaged spatial contours of pressure within the ensemble, which are shown in Fig. 6.13 for $v_{p}=50,250$ and $500 \mathrm{~m} / \mathrm{s}$. Values shown in the color-bar are in GPa. For high piston speeds, the pressure far exceeds $p_{c}$ behind the wave, and is fairly uniform as the amplitude of fluctuations is small relative to its average value.

Predicted profiles for the Cauchy stress components and the stress deviators are shown in Figs. 6.14(a) and (b), respectively, for $v_{p}=50,250$ and $500 \mathrm{~m} / \mathrm{s}$. For all piston speeds, $\overline{\boldsymbol{\sigma}}_{x x}$ is the largest of the normal stress components, which is expected for uniaxial waves. However, the magnitude of the normal stress components nearly equlibriate for high piston speeds. Because their average values must approach $\bar{p}$ by definition, it further implies that the average deviatoric stresses must relax to zero in the limit of high speed impact. This observation is evident in Fig. 6.14(b) where the deviatoric stress components remain largely uniform behind the compaction 
$50 \mathrm{~m} / \mathrm{s}$
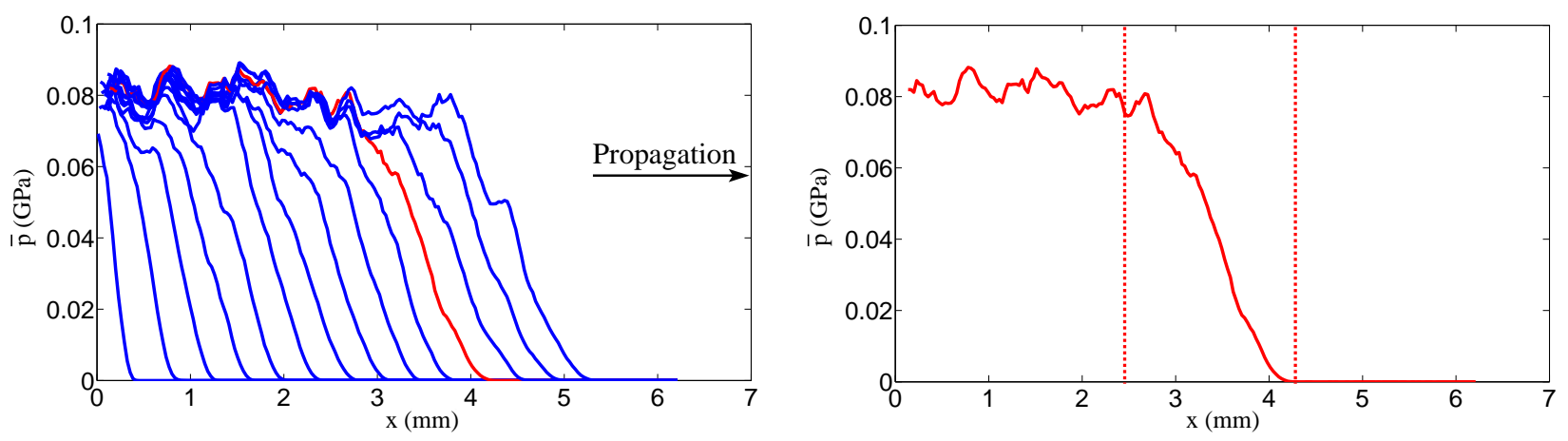

$250 \mathrm{~m} / \mathrm{s}$
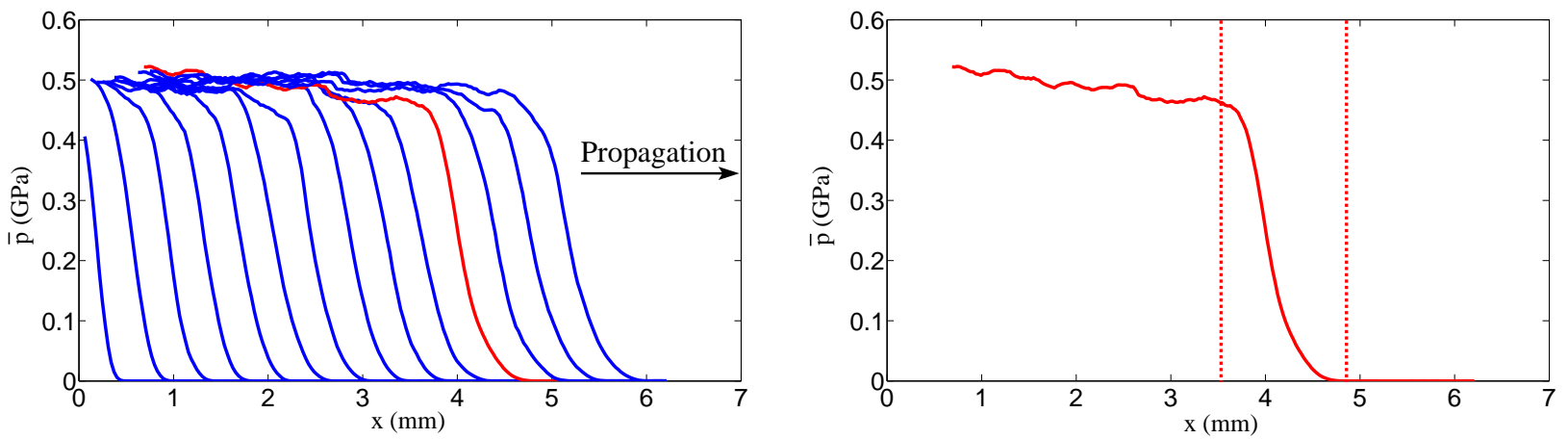

$500 \mathrm{~m} / \mathrm{s}$

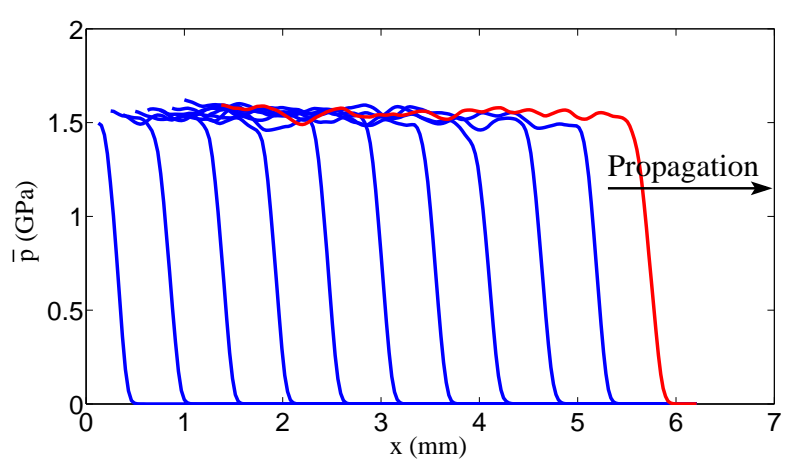

(a)

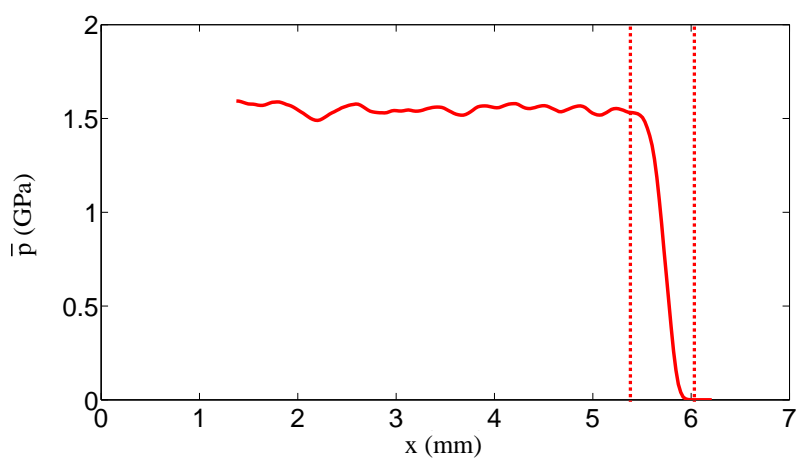

(b)

Figure 6.12: (a) Predictions for the temporal variation of the average pressure $\bar{p}$ for $v_{p}=50,250$ and $500 \mathrm{~m} / \mathrm{s}$. Profiles are shown in time-steps of $0.25 \mu \mathrm{s}$. (b) Predictions for the average pressure $\bar{p}$ at $2.75 \mu \mathrm{s}$ for $v_{p}=50,250$ and $500 \mathrm{~m} / \mathrm{s}$. 

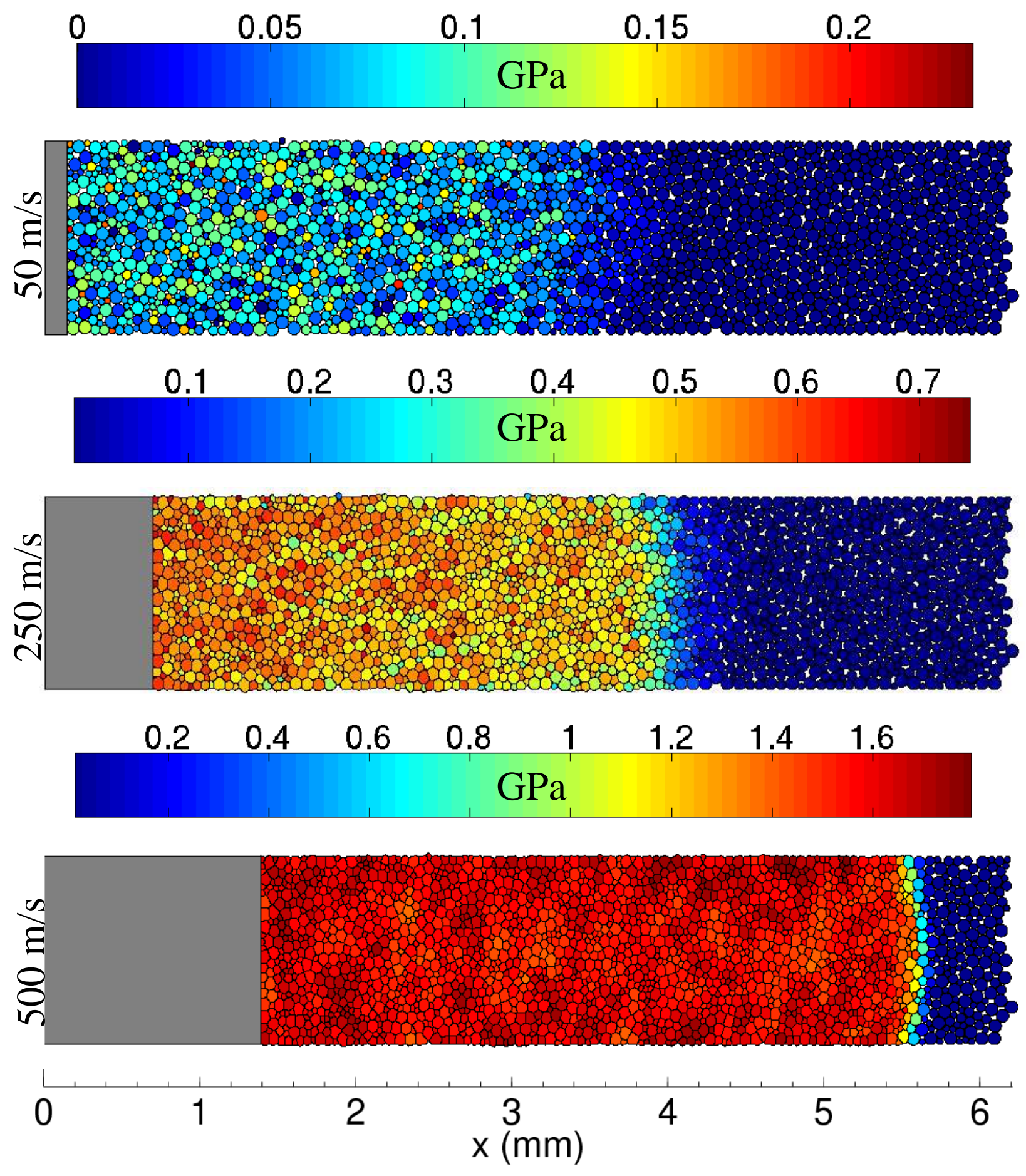

Figure 6.13: Predicted particle averaged pressure contours at $2.75 \mu \mathrm{s}$ for $v_{p}=50,250$ and $500 \mathrm{~m} / \mathrm{s}$. Values shown in the color-bar are in GPa. 
wave for $v_{p}=50$ and $250 \mathrm{~m} / \mathrm{s}$, whereas significant relaxation in the deviatoric components is observed for the $500 \mathrm{~m} / \mathrm{s}$ case. Similar observations were made by Menikoff [95] for granular HMX. Visco-elastic continuum models $[99,100]$ have been used to describe this bulk relaxation behavior in porous materials. The magnitude of deviatoric stresses are capped by the pressure independent yield criterion. Therefore, at high piston speeds, the pressure substantially exceeds the deviatoric components indicating that, in an average sense, the mechanical response approaches hydrodynamic behavior; indeed, this observation has motivated the exclusion of deviatoric stresses in most bulk compaction models [48].

Figure 6.15(a) shows predicted profiles for the average Von Mises stress $\overline{\boldsymbol{\tau}}_{e}$ for $v_{p}=50$ and $500 \mathrm{~m} / \mathrm{s}$ in time increments of $0.25 \mu \mathrm{s}$. The corresponding profiles at $t=2.75 \mu \mathrm{s}$ are shown in Fig. 6.15(b). The dotted horizontal line represents the initial particle yield strength $\tau_{0}=0.37$ GPa. For $v_{p}=50 \mathrm{~m} / \mathrm{s}$, the quasi-equilibrium value $\overline{\boldsymbol{\tau}}_{e}=0.15 \mathrm{GPa}$ behind the wave is well below the yield strength indicating that little plasticity occurs for low piston speeds, being largely confined to the immediate vicinity of particle contact surfaces. However, for $v_{p}=500 \mathrm{~m} / \mathrm{s}$, the profile for $\overline{\boldsymbol{\tau}}_{e}$ exceeds the value of $\tau_{0}$ because of rate-dependent plasticity. Unlike rate independent descriptions, the Von Mises stress is not confined to the yield surface; rather, it can overshoot and relax back to the yield surface, where the extent of overshoot increases with plastic viscosity. The profile for $\overline{\boldsymbol{\tau}}_{e}$ subsequently relaxes to well below the yield surface behind the wave because of relaxation of the deviatoric stress components.

Figure 6.16 summarizes profiles for the average effective plastic strain $\bar{\epsilon}_{p}$ for all piston speeds. Also shown in the figure is the prediction corresponding to $v_{p}=250 \mathrm{~m} / \mathrm{s}$. Here, $\bar{\epsilon}_{p}$ increases with piston speed, and is maximum near the piston for each case. The predictions indicate that plastic strain vanishes within the compaction zone; this location denotes the terminal end precursor region. Within the precursor region, deformation is mostly elastic, and dissipation is largely due to interparticle friction. Profiles for $\overline{\boldsymbol{\tau}}_{e}$ and $\bar{\epsilon}_{p}$ collectively highlight the spatial variation of plastic deformation within the wave structure and its dependence on piston speed. Temperature rise resulting from plastic work depends on $\bar{\epsilon}_{p}$ through the yield strength $\tau_{0}$, density $\rho$, and specific 
$50 \mathrm{~m} / \mathrm{s}$
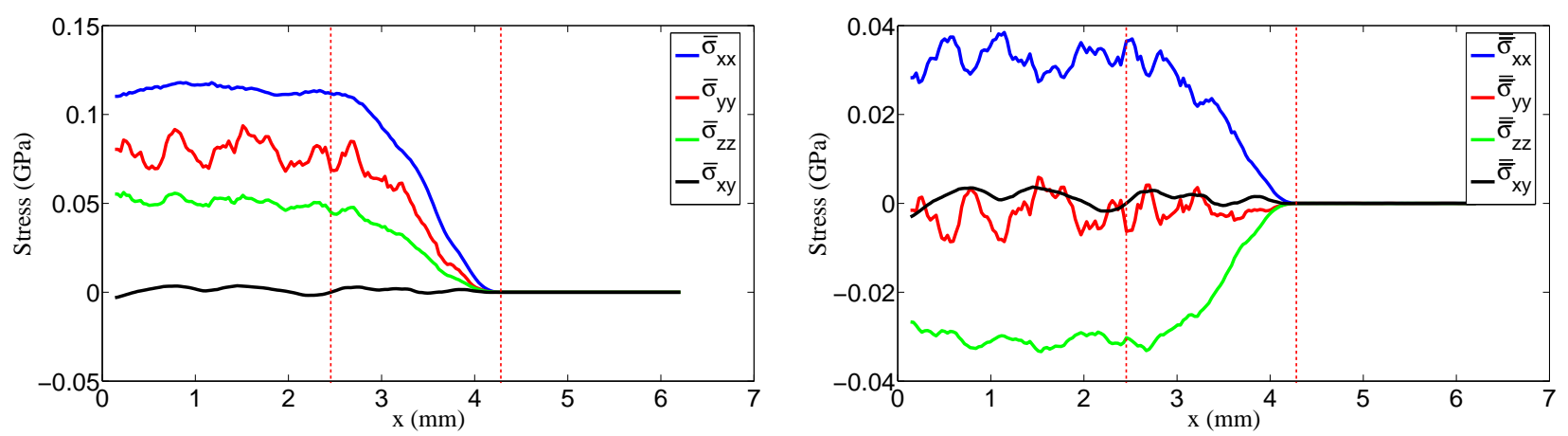

$250 \mathrm{~m} / \mathrm{s}$
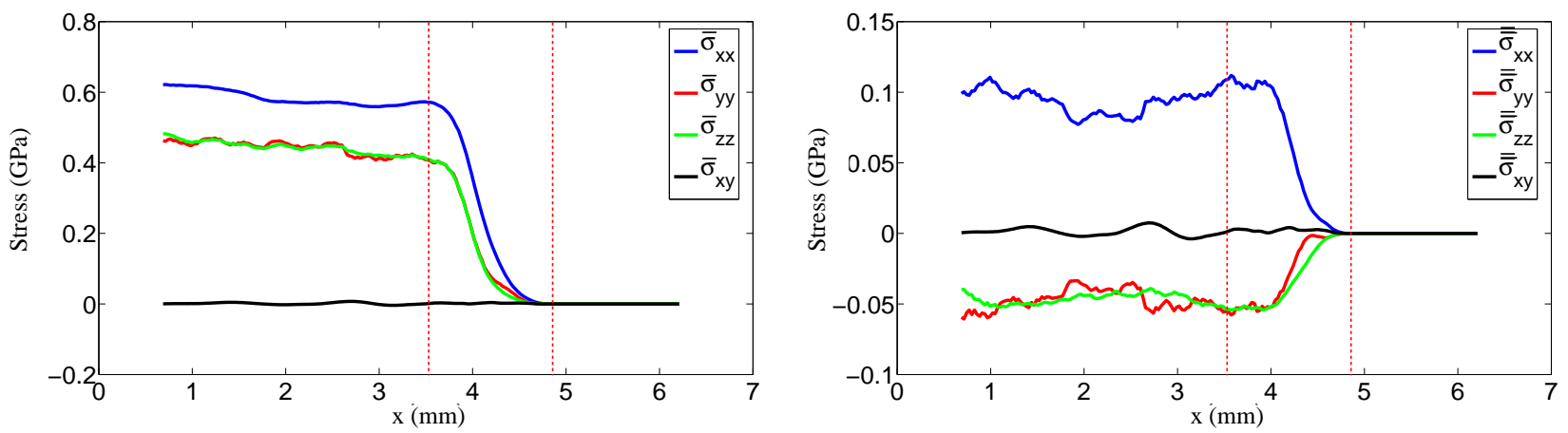

$500 \mathrm{~m} / \mathrm{s}$

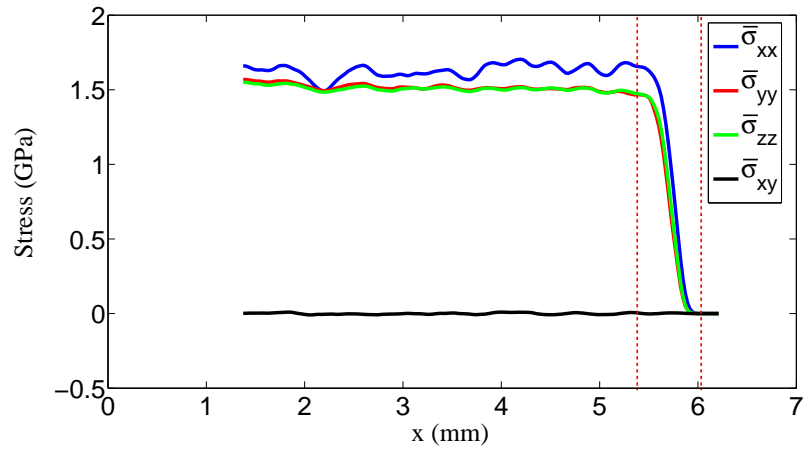

(a)

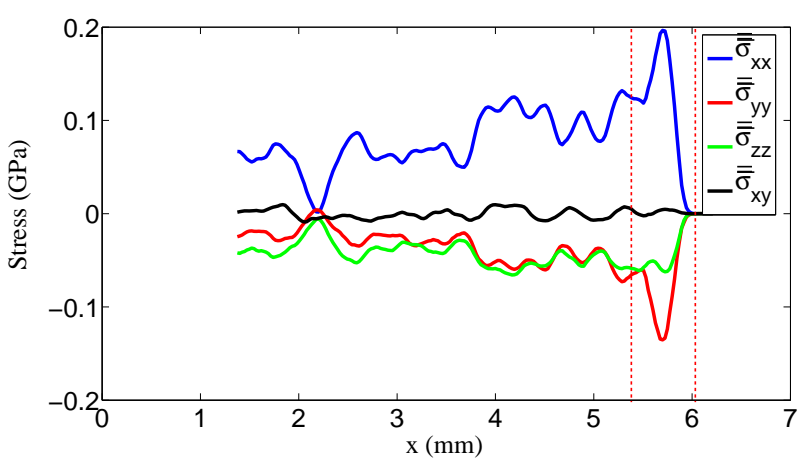

(b)

Figure 6.14: Predictions for the average (a) Cauchy stress components $\overline{\boldsymbol{\sigma}}$ and (b) deviatoric stress components $\overline{\overline{\boldsymbol{\sigma}}}$ at $2.75 \mu \mathrm{s}$ for $v_{p}=50,250$ and $500 \mathrm{~m} / \mathrm{s}$. 
$50 \mathrm{~m} / \mathrm{s}$
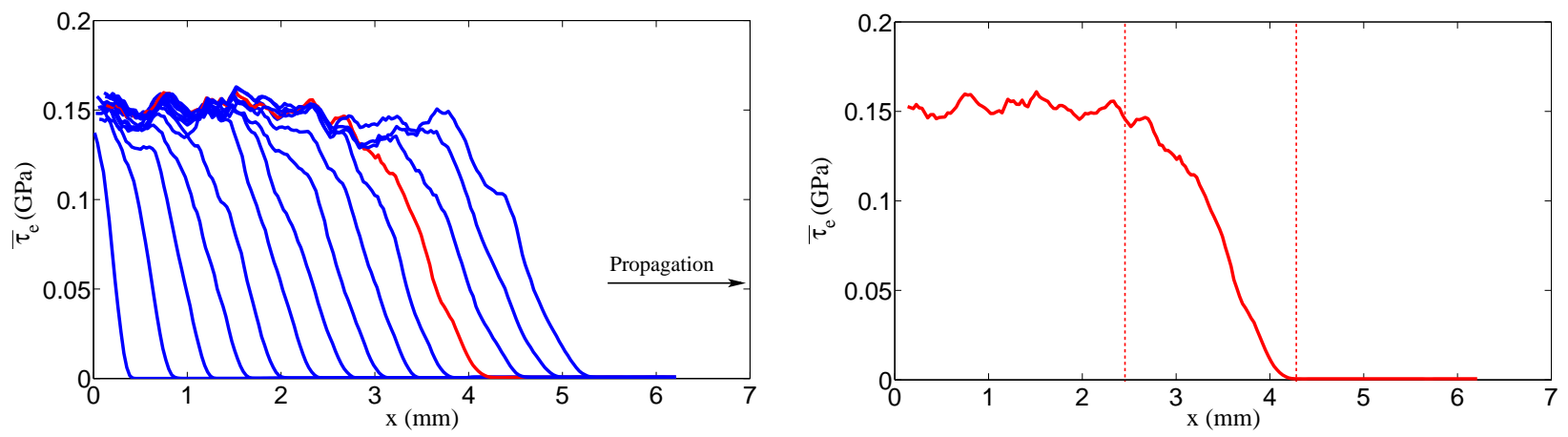

$500 \mathrm{~m} / \mathrm{s}$

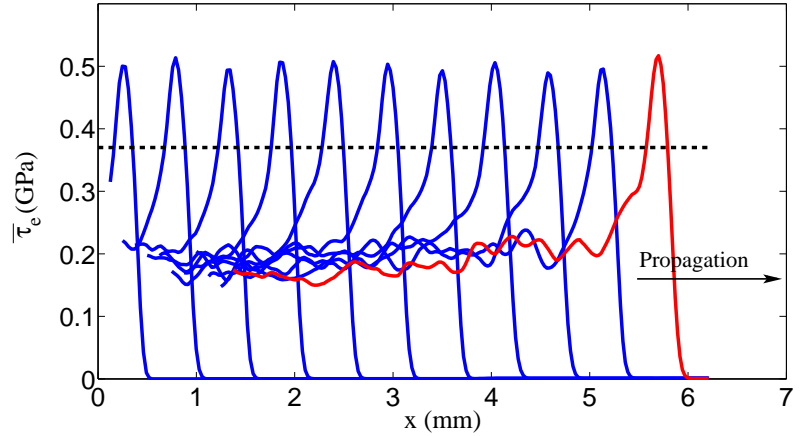

(a)

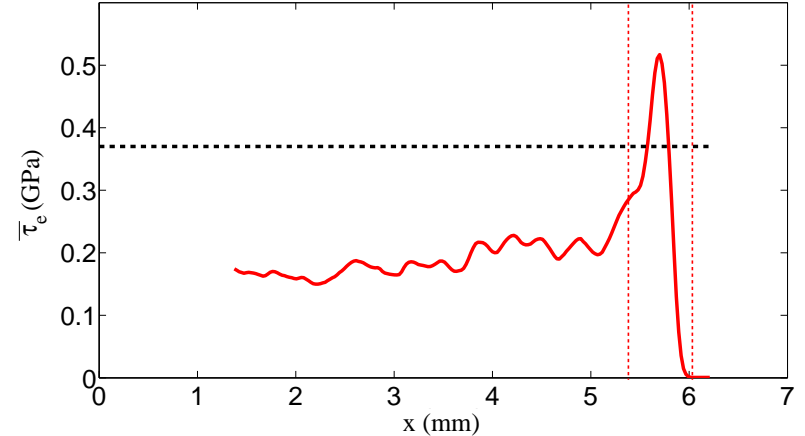

(b)

Figure 6.15: (a) Predictions for the temporal variation of the average Von Mises stress $\overline{\boldsymbol{\tau}}_{e}$ for $v_{p}$ $=50$ and $500 \mathrm{~m} / \mathrm{s}$. Profiles are shown in time-steps of $0.25 \mu \mathrm{s}$. (b) Predictions for the average Von Mises stress $\overline{\boldsymbol{\tau}}_{e}$ at $2.75 \mu \mathrm{s}$ for $v_{p}=50$ and $500 \mathrm{~m} / \mathrm{s}$.

heat $c_{v}$, because $\Delta T_{p} \approx \bar{\epsilon}_{p} \tau_{0} / \rho c_{v}$, with $\rho \approx \bar{\rho}$. For $v_{p}=500 \mathrm{~m} / \mathrm{s}$, this relation predicts a maximum average temperature rise of only $15 \mathrm{~K}$. However, as will be shown later, localized plastic dissipation near particle contact surfaces leads to much higher temperature rises $(\Delta T \geq 100 \mathrm{~K})$, even for low speed impact.

Figure 6.17 shows finite element contours for $\tau_{e}$ at $t=2.75 \mu \mathrm{s}$ for $v_{p}=50,250$, and $500 \mathrm{~m} / \mathrm{s}$. Values shown in the color-bars are in GPa. These contours provide detailed information about stress propagation through the particle ensemble, and highlight key qualitative and quantitative differences in wave structure for different piston speeds. Particle positions are shown in a laboratory frame, and piston displacements are indicated by shaded boxes. For low piston speeds, a weak, dispersed compaction wave structure is predicted. The head of the wave is difficult to identify, and no discernible wave structure exists. Here, stress is transmitted in preferential directions 


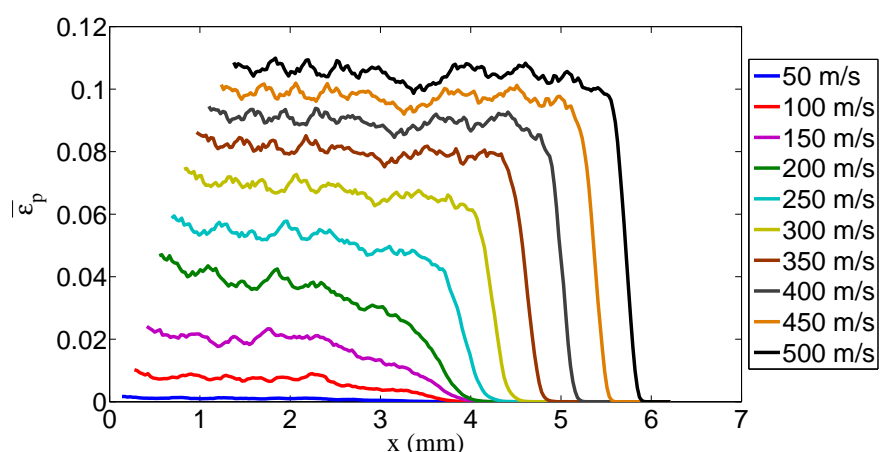

(a)

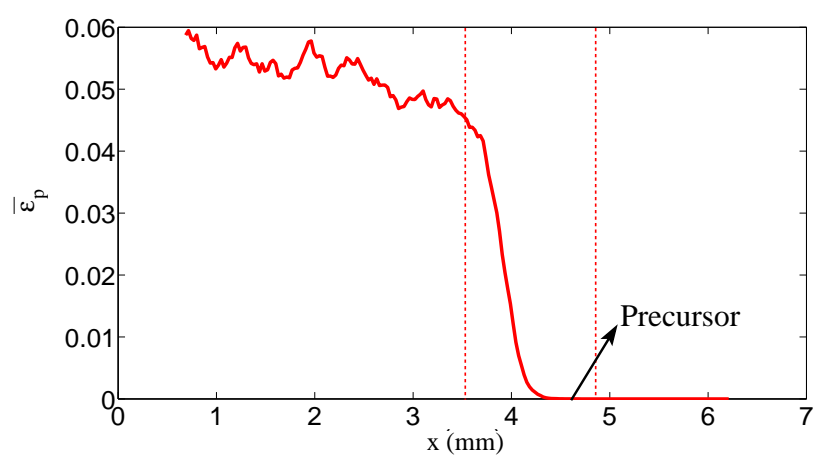

(b)

Figure 6.16: Predictions for the average effective plastic strain $\bar{\epsilon}_{p}$ at $2.75 \mu$ s for (a) $v_{p}=50-500 \mathrm{~m} / \mathrm{s}$ (b) $v_{p}=250 \mathrm{~m} / \mathrm{s}$.

by interparticle contact giving rise to significant stress bridging within the meso-structure; these stress bridges correspond to high stress pathways that are mostly aligned in the axial direction. Particles not involved in these pathways experience little stress, whereas particles involved experience stress concentrations and large plastic deformation, even for low piston speeds. A maximum value of approximately $\tau_{e}=0.55 \mathrm{GPa}$ is predicted for $v_{p}=50 \mathrm{~m} / \mathrm{s}$. This value is significantly larger than the corresponding average value of $\overline{\boldsymbol{\tau}}_{e}=0.15 \mathrm{GPa}$. A magnified portion of the ensemble is shown in Fig. 6.18 to highlight the stress chains. The wave strength increases with piston speed, and the two region (precursor/trailer) structure is apparent for $v_{p} \geq 250 \mathrm{~m} / \mathrm{s}$, but is difficult to observe in the plot for $v_{p}=500 \mathrm{~m} / \mathrm{s}$ because of the large range of stresses involved. Also, the wave front for $v_{p}=500 \mathrm{~m} / \mathrm{s}$ appears curved rather than planar due to transverse variations in local porosity generated by the particle settling algorithm. The porosity is slightly lower along the transverse boundaries than in the interior of the ensemble, resulting in slightly faster stress transmission within the interior.

In this study, wave speeds are computed based on data for $\widetilde{v}_{x}$. To this end, the axial positions of both the precursor and trailing wave are identified and recorded with time; data obtained for $v_{p}=250 \mathrm{~m} / \mathrm{s}$ are shown in Fig. 6.19(a). Here, points plotted in red and blue correspond to the precursor and trailing waves, respectively. Wave speeds are determined by a least squares fit to linear portions of the long-time data, where the slope of the line corresponds to the wave speed. In general, the data points correlate well with the linear fit indicating steady wave behavior. Scatter 


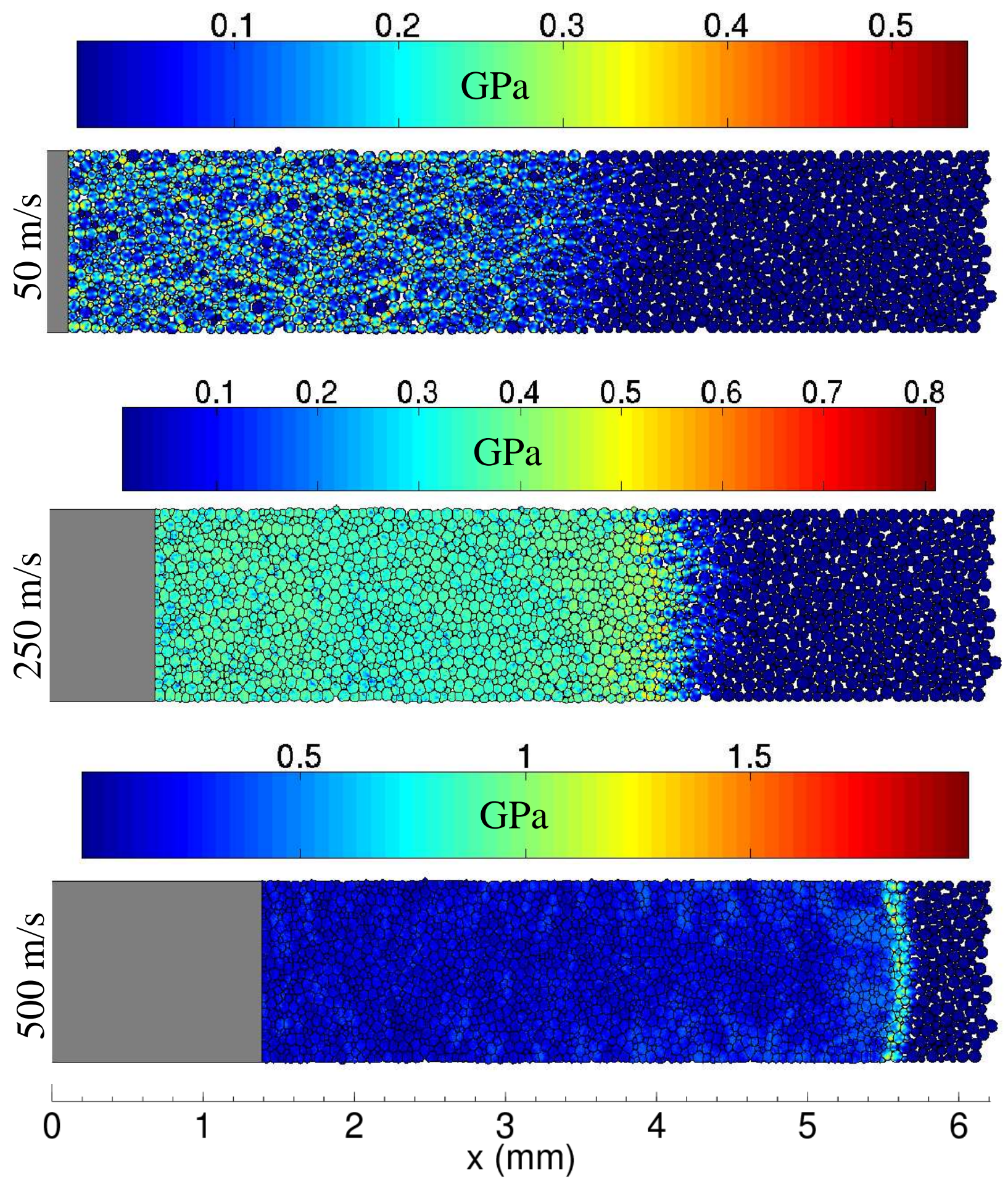

Figure 6.17: Predicted finite element contours of Von Mises stress $\boldsymbol{\tau}_{e}$ within particles at $2.75 \mu \mathrm{s}$ for $v_{p}=50,250$ and $500 \mathrm{~m} / \mathrm{s}$. Values shown in the color-bars are in GPa. 


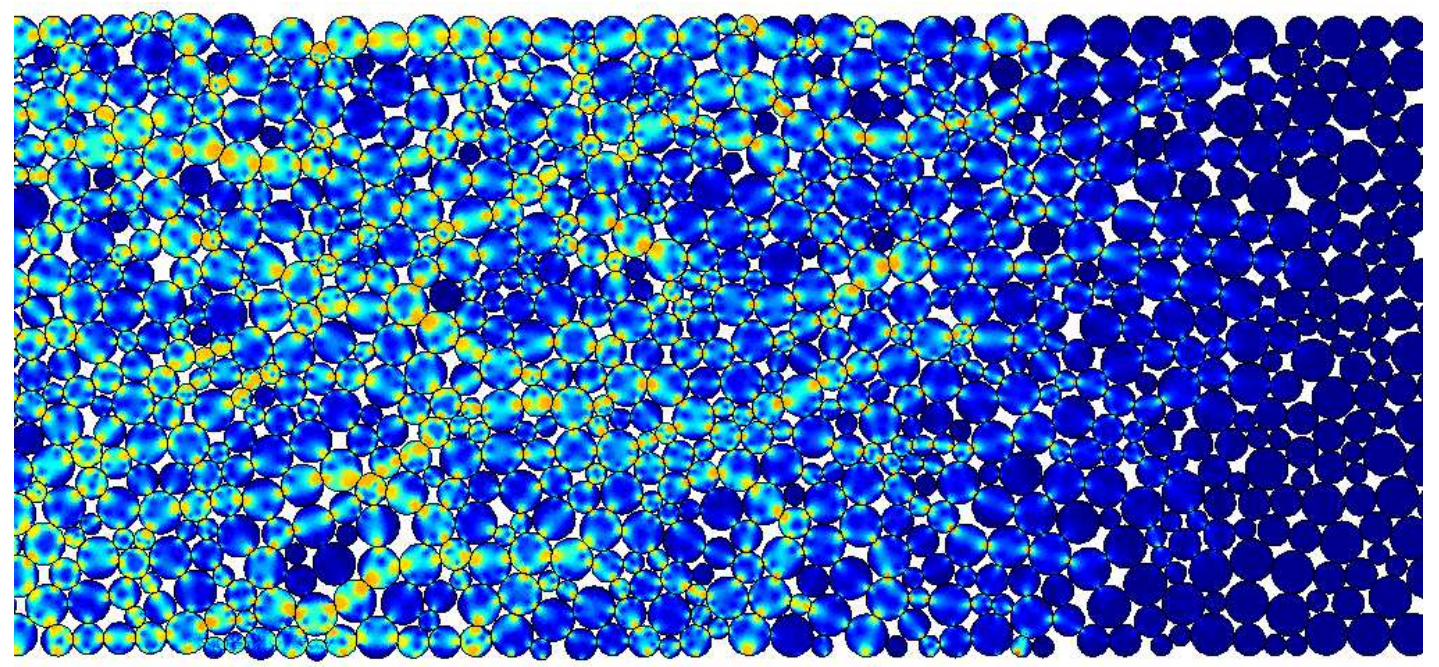

Figure 6.18: Magnified portion of the finite element contour of Von Mises stress $\boldsymbol{\tau}_{e}$ within particles at $2.75 \mu \mathrm{s}$ for $v_{p}=50 \mathrm{~m} / \mathrm{s}$.

in the data is largest for low piston speeds, and is probably due to initial impact transients. The variation in lead $\left(D_{l}\right)$ and trailing $\left(D_{t}\right)$ wave speed with piston speed, referred to as a Hugoniot plot [101], is summarized in Fig. 6.19(b). The wave speeds plateau between $100 \geq v_{p} \geq 250 \mathrm{~m} / \mathrm{s}$, and increase linearly with piston speed for $v_{p} \geq 250$. The precursor outruns the trailing wave for low piston speeds, and they coalesce for higher piston speeds. Steady-state compaction wave modeling performed by Gonthier [49] for strain hardened granular explosive showed qualitatively similar trends for the Hugoniot. For increasing piston speed, his steady analysis predicted wave structures consisting of: 1) a single viscoelastic wave whose speed linearly increases with piston speed up to a viscoelastic limit; 2) a viscoelastic precursor wave followed by a slower viscoplastic wave, where the viscoplastic wave speed increases linearly with piston speed while the precursor speed remains fixed; and 3) a single viscoplastic wave whose speed increases linearly with piston speed. These structures are qualitatively analogous to those described by classical theory for elastic-plastic impact of homogeneous solids, as discussed in Ref. [49].

Comparisons are given in Fig. 6.19 between the FDEM predictions and available experimental data for $D_{t^{-}} v_{p}$ and $\bar{p}_{f}-v_{p}$ Hugoniot curves, where $\bar{p}_{f}$ is the quasi-equilibrium value of the average pressure behind the wave. Four sets of data are shown; three data sets are for HMX and one is for PBX 9404. The explosive PBX-9404 is a HMX based Plastic Bonded explosive consisting of 


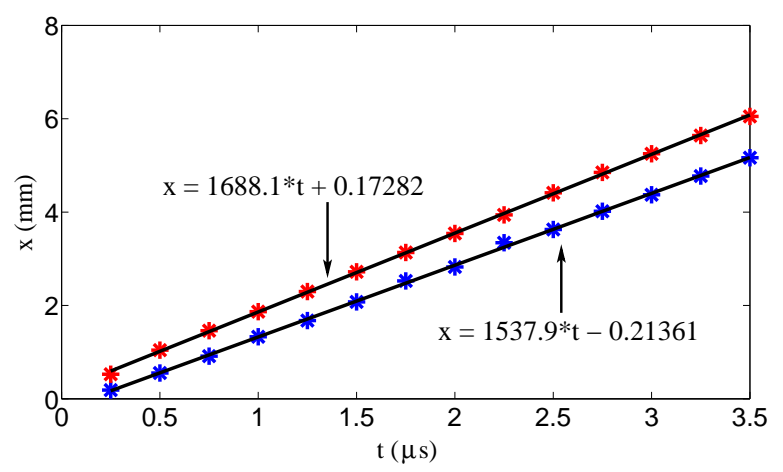

(a)

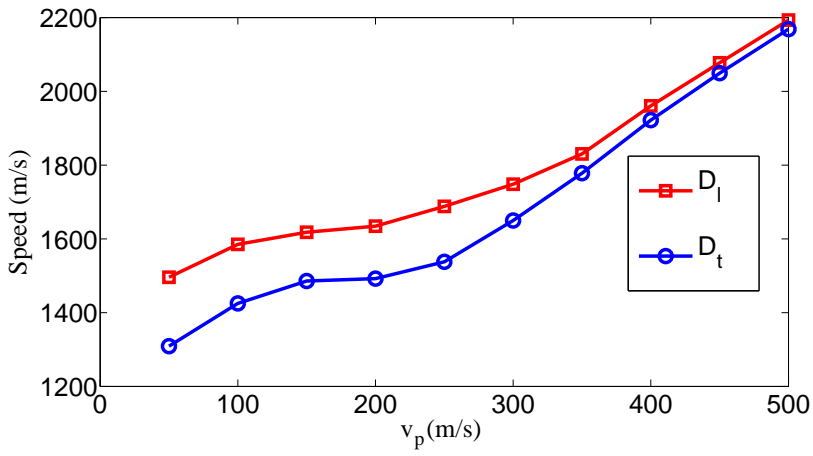

(b)

Figure 6.19: (a) Predicted wave speed for $v_{p}=250 \mathrm{~m} / \mathrm{s}$. (b) Predicted variation in precursor $\left(D_{l}\right)$ and plastic $\left(D_{t}\right)$ wave speed with piston speed $\left(v_{p}\right)$.

95\% HMX, 2.5\% estane, and 2.5\% of bisdinitropropyl acetal-bisdinitropropyl formal eutectic by weight. Percentages indicated in the legends represent initial bulk solid volume fraction $\phi_{s, b}$. For fixed piston speed, both $D_{t}$ and $\bar{p}_{f}$ increase with $\phi_{s, b}$ due to enhanced stress transmission between tightly packed particles. Because it is well established that particle morphology significantly influences deformation wave speed and strength [85], data obtained from one experiment may not correlate well with data obtained from other experiments conducted under similar loading conditions but with different particle size distributions and shapes. Making comparisons with 2D numerical predictions are even more cumbersome due to dimensional effects. Despite these difficulties, comparisons indicate that the FDEM predictions qualitatively agree with measured data.

To ensure that the bulk mechanical response of the particle ensemble is independent of domain size and that the compaction wave behavior is indeed representative of quasi-steady behavior, simulations were also performed on a larger particle ensemble comprising of 4000 particles. The alternate ensemble had the same particle size distribution and initial porosity, but its domain was $1.5 \mathrm{~mm}$ in width and $10.34 \mathrm{~mm}$ in length, which allowed for the equations of motion to be integrated through a total time of $6 \mu \mathrm{s}$ before the wave reached the open end of the domain. Comparisons of the variation in wave-speed and quasi-steady bulk pressure with piston speed between the current and alternate particle ensemble are presented in Fig. 6.21. Also shown in the figure are the predictions by Gonthier [49] from his continuum mechanics bulk compaction model. 


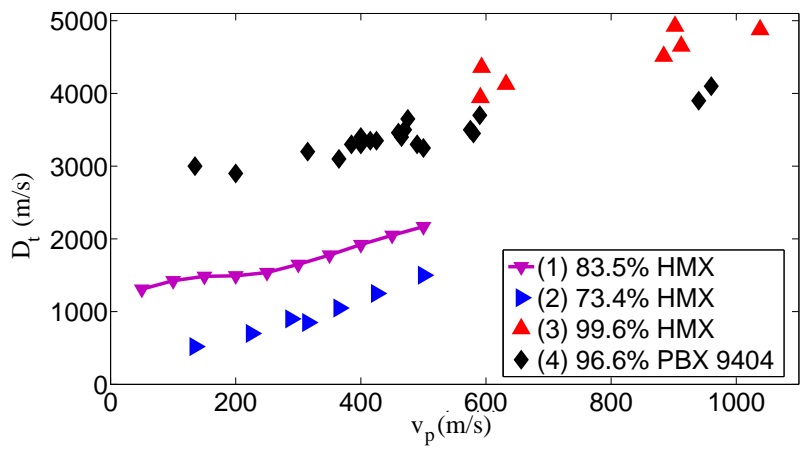

(a)

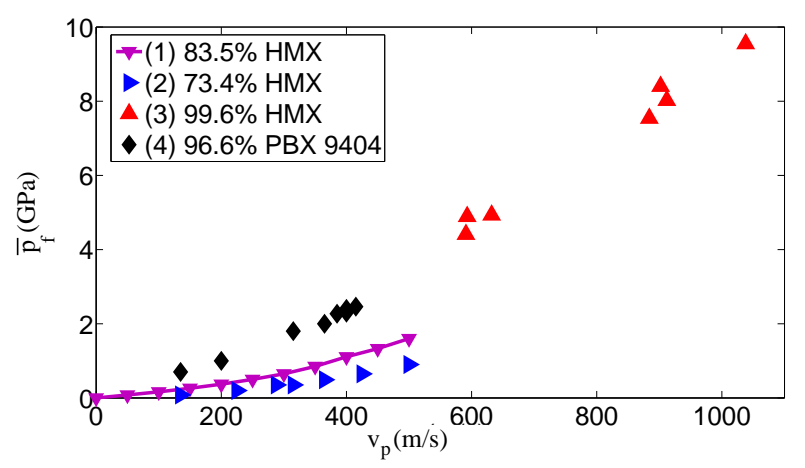

(b)

Figure 6.20: Hugoniot for (a) wave-piston speed and (b) pressure-piston speed. (1) Numerical predictions from the FDEM (2) Experimental data from ref. [121]. (3) Experimental data from ref. [92]. (4) Experimental data from ref. [121].

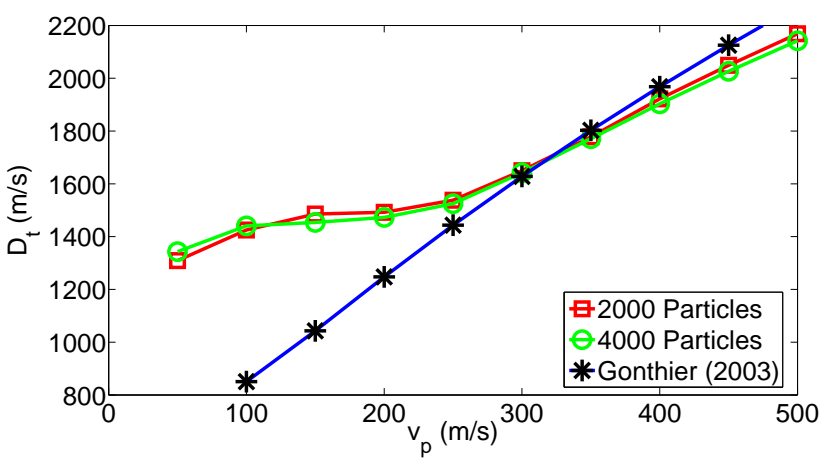

(a)

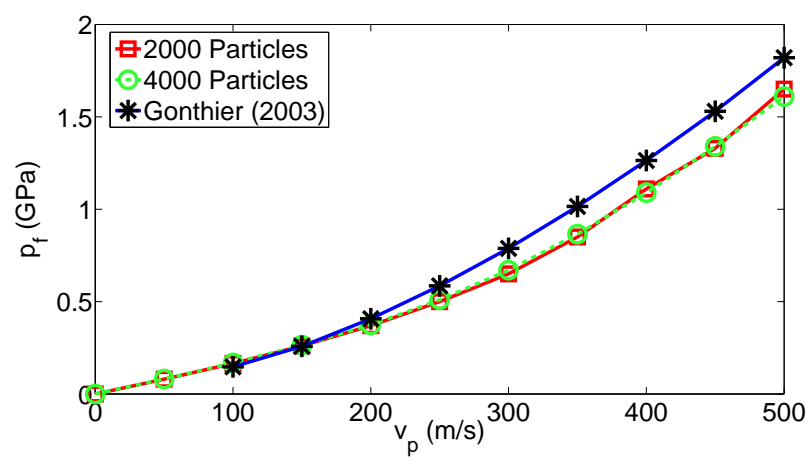

(b)

Figure 6.21: Comparison of the Hugoniot for (a) wave-piston speed and (b) pressure-piston speed between the 2000 and 4000 particle ensemble.

From the figures it is evident that the Hugoniots for both ensembles display similar qualitative and quantitative trends confirming that the wave behavior is indicative of quasi-steady behavior and independent of domain size. In comparison with the bulk predictions, the predictions from the FDEM under predict the wave speeds at low piston speeds $\left(v_{p} \leq 300 \mathrm{~m} / \mathrm{s}\right)$ but match the bulk predictions at higher piston speeds. Predictions for the quasi-steady pressure from the bulk and meso-scale models are in good qualitative and quantitative agreement.

\subsubsection{Thermal Response}

The thermal response of the particle ensemble is now addressed. In this section, predicted variations in particle energetics through the compaction wave structure are first characterized, 


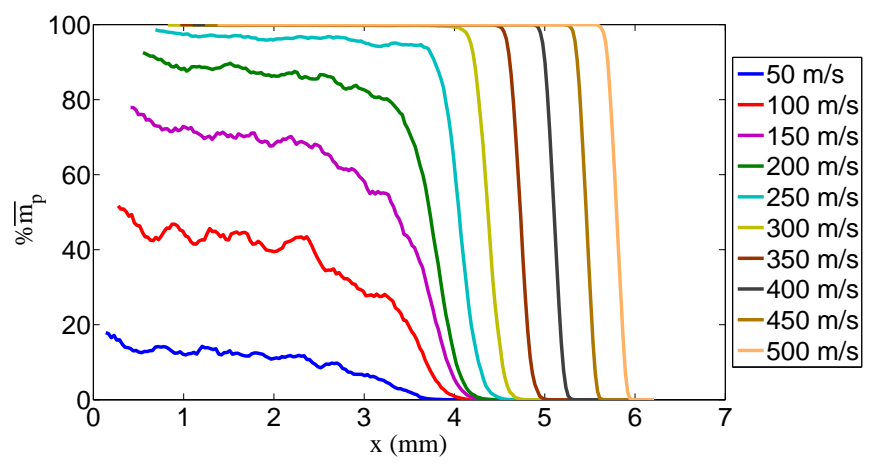

(a)

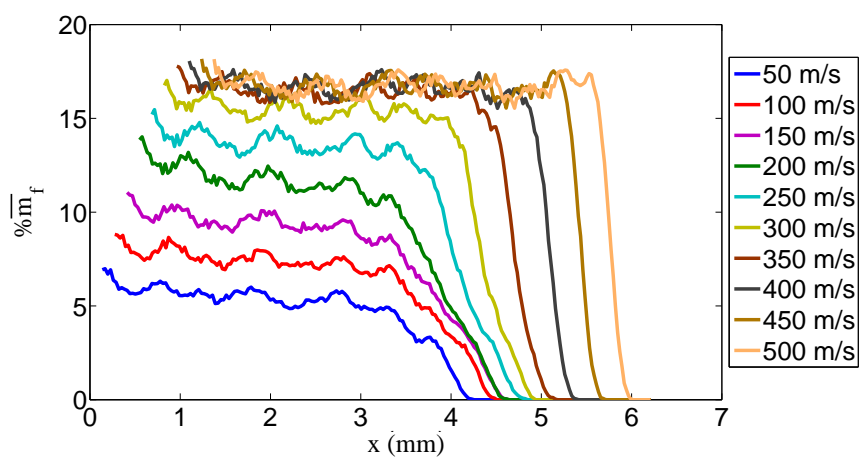

(b)

Figure 6.22: Predictions for the (a) plastic mass-fraction $\bar{m}_{p}$ and (b) friction mass fraction $\bar{m}_{f}$ at $2.75 \mu \mathrm{s}$ for $v_{p}=50-500 \mathrm{~m} / \mathrm{s}$.

followed by an analysis of hot-spot temperature and mass fractions. Particular emphasis is placed on identifying the relative importance of plastic and friction work as hot-spot mechanisms that may locally ignite the material.

\section{- Energetics}

Figure 6.22 summarizes the percentage of mass that is locally influenced by plastic and friction work within the compaction zone for different piston speeds. These plots are intended to highlight the extent of bulk plastic dissipation versus locally dissipated energy by friction. The local plastic mass-fraction $\bar{m}_{p}$ is estimated by taking the ratio of the instantaneous mass affected by plastic work within an averaging area to the total mass within that area. Because friction is a surface phenomenon, the mass directly influenced by friction work is zero. However, for purposes of comparison with plastic work, friction work is locally distributed to finite elements that are directly connected to nodes affected by the frictional heat flux; this assumption is reasonable because thermal conduction has little influence over the fast time scales of these simulations. For all speeds, $\bar{m}_{p}$ and $\bar{m}_{f}$ are maximum near the piston boundary. For $v_{p}=50 \mathrm{~m} / \mathrm{s}$, only $18 \%$ of local mass experiences plasticity, which increases to $100 \%$ for $v_{p} \geq 300 \mathrm{~m} / \mathrm{s}$. Less than $7 \%$ of the local mass undergoes friction work for $v_{p}=50 \mathrm{~m} / \mathrm{s}$, which increases to $17 \%$ for $v_{p} \geq 400 \mathrm{~m} / \mathrm{s}$.

The partitioning of total system energy into potential energy $\left(W_{e}\right)$, kinetic energy $\left(E_{k}\right)$, plastic work $\left(W_{p}\right)$, and friction work $\left(W_{f}\right)$ is now considered. To this end, average profiles of $\bar{W}_{e}, \bar{W}_{p}$, 
$\bar{W}_{f}$, and $\bar{E}_{k}$ are plotted in Fig. 6.23(a) for $v_{p}=50,250$, and $500 \mathrm{~m} / \mathrm{s}$. Predictions in this figure indicate that kinetic energy dominates the energy response for all piston speeds, while friction work is smallest. This prediction is expected because friction work is localized to contact surfaces. Both plastic work and friction work increase with $v_{p}$, with the maximum dissipation due each occurring at the piston surface. Though energy dissipated by plastic work is substantially larger than by friction work, it is difficult to characterize their relative importance based on total work components because considerably more mass experiences plastic work. Therefore, it is instructive to consider mass-specific energy and work components, which are plotted in Fig. 6.23(b). Here, profiles for each energy/work component are estimated based on the local mass that experiences that form of energy/work. For example, mass-specific plastic work within an averaging area is estimated as the ratio of the net plastic work to the instantaneous mass affected by plastic work. Here, two observations are noteworthy. First, friction work $\left(\bar{w}_{f}\right)$ dominates plastic work $\left(\bar{w}_{p}\right)$ for $v_{p}=50 \mathrm{~m} / \mathrm{s}$, but they are comparable for $v_{p}=250 \mathrm{~m} / \mathrm{s}$. This prediction indicates that friction work may be a more important hot-spot mechanism than plastic work for low speed impact. Second, both $\bar{W}_{f}$ and $\bar{m}_{f}$ vary substantially with axial position due to heterogeneities within the meso-structure. Thus, high frequency oscillations result in $\bar{w}_{f}$ indicating that friction work is sensitive to the meso-structure. This prediction is important because both static and dynamic compaction experiments on HMX [84] reveal substantial particle fracture which introduces additional heterogeneities within the meso-structure. It is our belief that fracture, and subsequent frictional rearrangement of particles, is a primary hot-spot mechanism that can initiate DDT, especially for low impact speeds.

The relative importance of plastic and friction work can be further examined by considering average profiles for the plastic and frictional heating rates, which are shown in Fig. 6.24 for $v_{p}=50$, 250, and $500 \mathrm{~m} / \mathrm{s}$. In these plots, $\overline{\dot{w}}_{p}$ and $\overline{\dot{w}}_{f}$ represent average mass-specific plastic and frictional heating rates. These profiles have a similar pulse-like structure to $\widetilde{v}_{x}$ because the heating rates largely vanish in the ambient and quasi-equilibrium final states. The maximum heating rate, given by the peak of the pulse, occurs within the compaction zone. Both $\overline{\dot{w}}_{p}$ and $\overline{\dot{w}}_{f}$ increase with piston 
$50 \mathrm{~m} / \mathrm{s}$
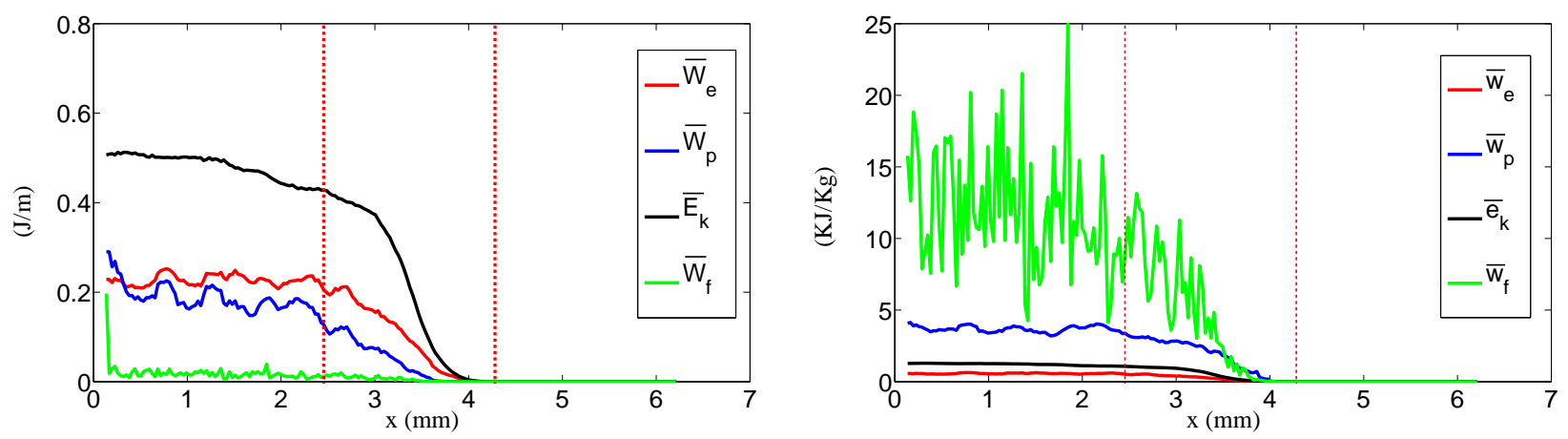

$250 \mathrm{~m} / \mathrm{s}$
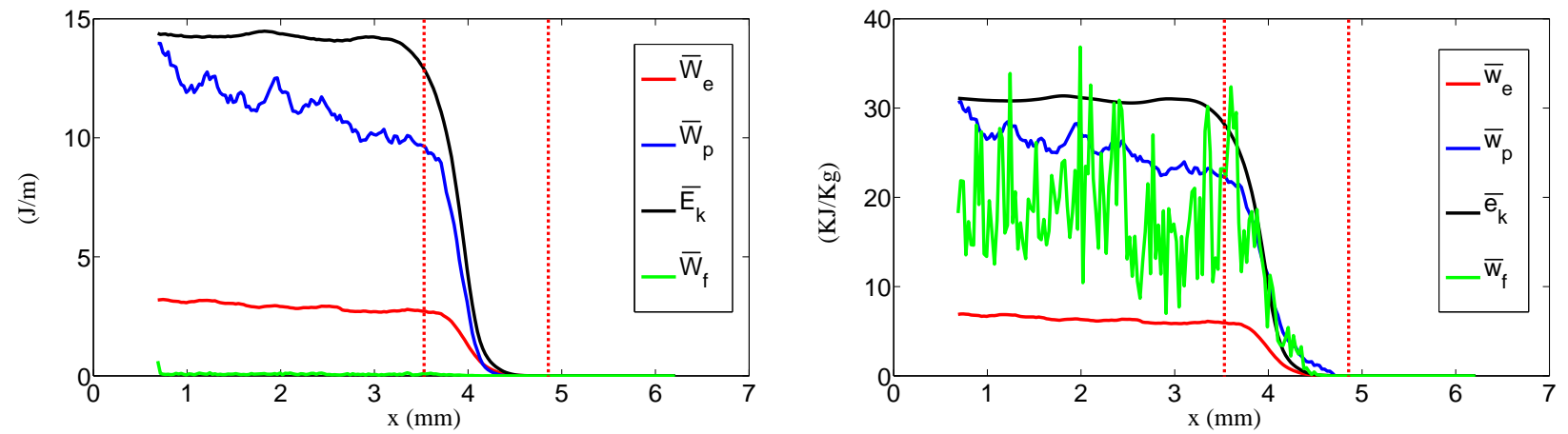

$500 \mathrm{~m} / \mathrm{s}$

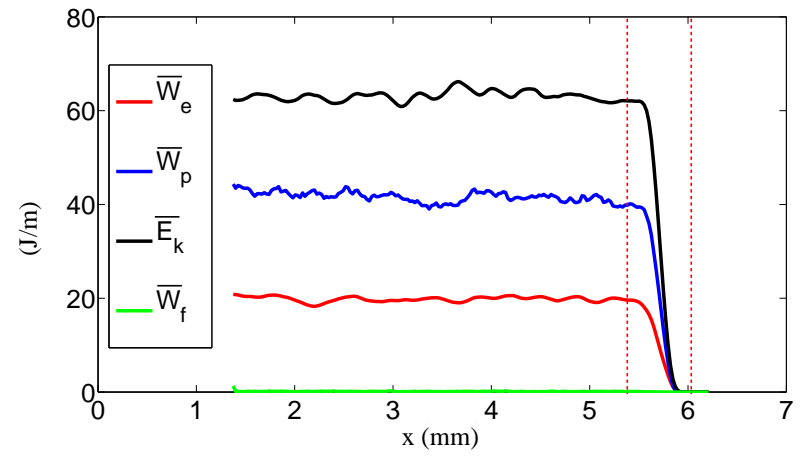

(a)

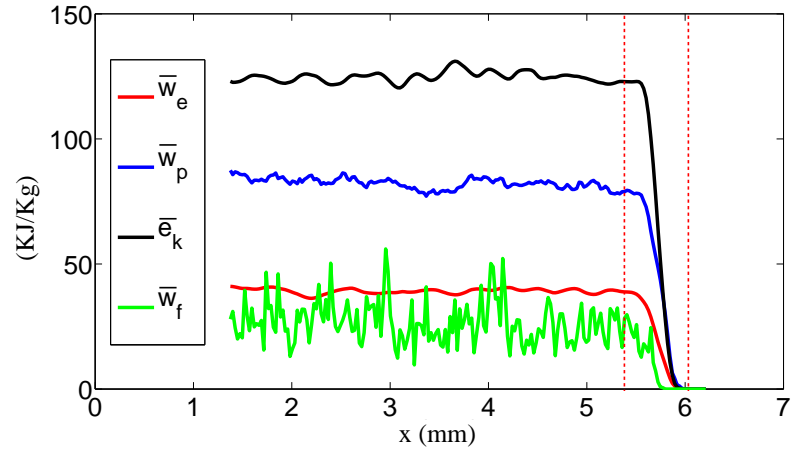

(b)

Figure 6.23: Predictions for the partitioning of (a) total system energy and (b) mass-specific system energy at $2.75 \mu \mathrm{s}$ for $v_{p}=50,250$ and $500 \mathrm{~m} / \mathrm{s}$. 
speed, and are comparable for low to moderate piston speeds. For higher piston speeds, the plastic heating rate substantially exceeds the frictional heating rate. The peak heating rates both decay with time, with maximal heating occurring close to the piston boundary immediately following impact. Both quantities exhibit substantial temporal variations at low piston speeds due to the influence of heterogeneities, but approach nearly uniform profiles for $v_{p}=500 \mathrm{~m} / \mathrm{s}$. For fixed time, the average frictional heating rate leads the average plastic heating rate because plastic heating is minimal within the precursor region of the wave. It is possible that frictional heating within the precursor can seed heat particles prior to the onset of more extensive heating induced by the trailing, plastic region of the wave.

\section{- Temperature Response}

The temperature response of the particle ensemble is now described. Figure 6.25(a) summarizes predicted average temperature profiles $\bar{T}$ for all piston speeds considered. Here, two observations are noteworthy. First, profiles of $\bar{T}$ correlate very well with the predicted average plastic strain $\bar{\epsilon}_{p}$ profiles shown in Fig. 6.16. Figure 6.25(c) illustrates a linear variation of $\bar{T}$ with $\bar{\epsilon}_{p}$ within the compaction zone, where the slope depends on $v_{p}$. The cluster of points occurring at the end of each line correspond to the data within the long quasi-steady compacted region behind the wave where $\bar{T}$ and $\bar{\epsilon}_{p}$ are largely constant. This prediction indicates that the average temperature is largely the result of volumetric plastic heating. Second, Fig. 6.25(a) indicates that even for $v_{p}=500 \mathrm{~m} / \mathrm{s}$, the predicted maximum average temperature rise is well below the ignition threshold for HMX (ignition temperature $~ 600 \mathrm{~K}$ ). However, DDT experiments have shown that ignition occurs for impact speeds as low as $100 \mathrm{~m} / \mathrm{s}$. This observation can be explained by considering the local variation in peak temperature within the compaction zone, as shown in Fig. 6.25(b). These temperatures are the maximum values that locally occur within averaging areas throughout the wave; the staircase appearance in this plot is reflective of the averaging area length. The figure indicates that local mass is heated to substantially higher temperatures than that suggested by the average temperature profiles of Fig. 6.25(a). Maximum temperatures of approximately $600 \mathrm{~K}$ occur in localized regions within the ensemble, even for $v_{p}=50 \mathrm{~m} / \mathrm{s}$, which 
$50 \mathrm{~m} / \mathrm{s}$
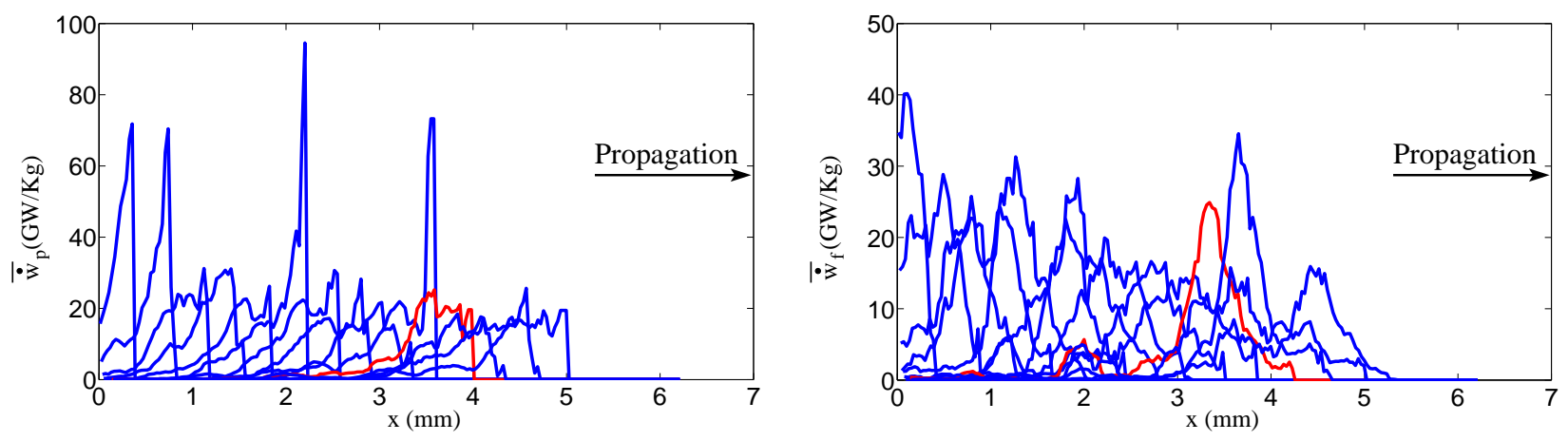

$250 \mathrm{~m} / \mathrm{s}$
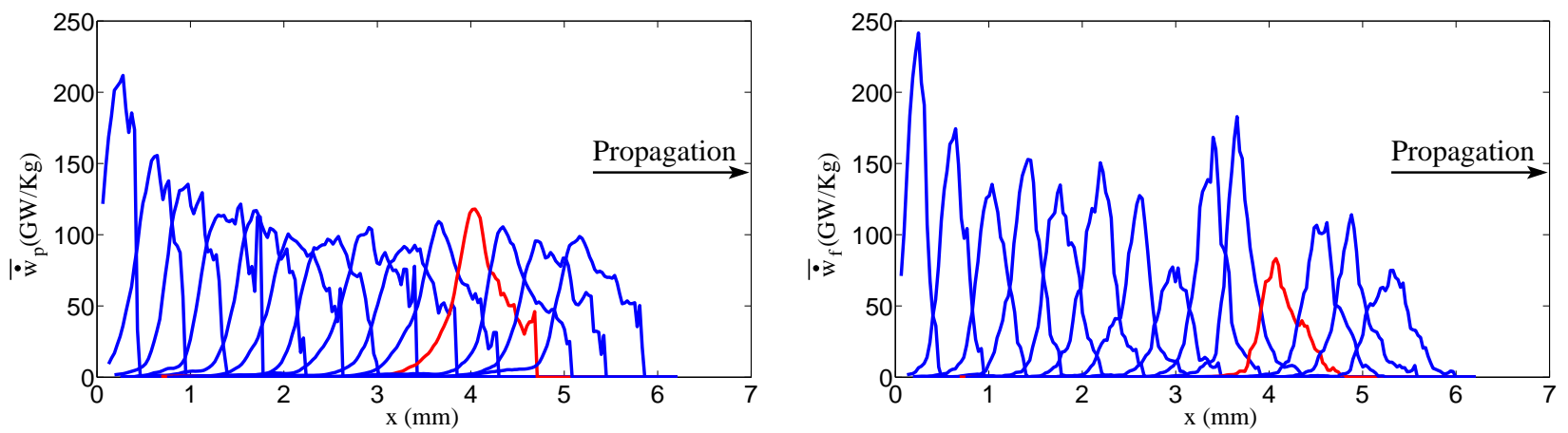

$500 \mathrm{~m} / \mathrm{s}$

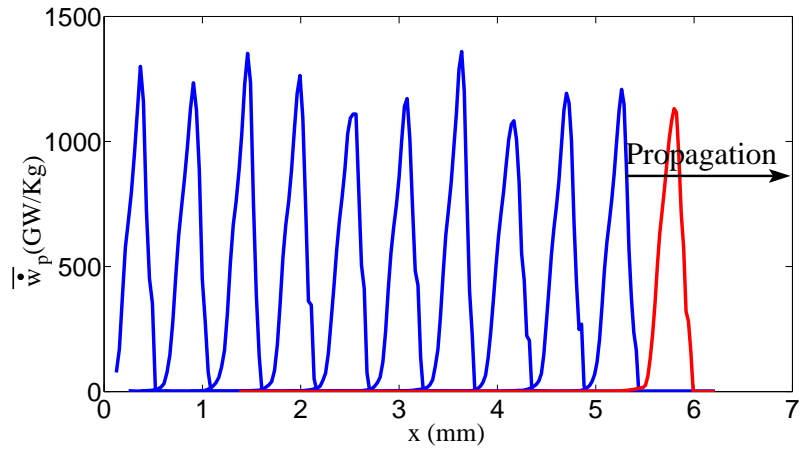

(a)

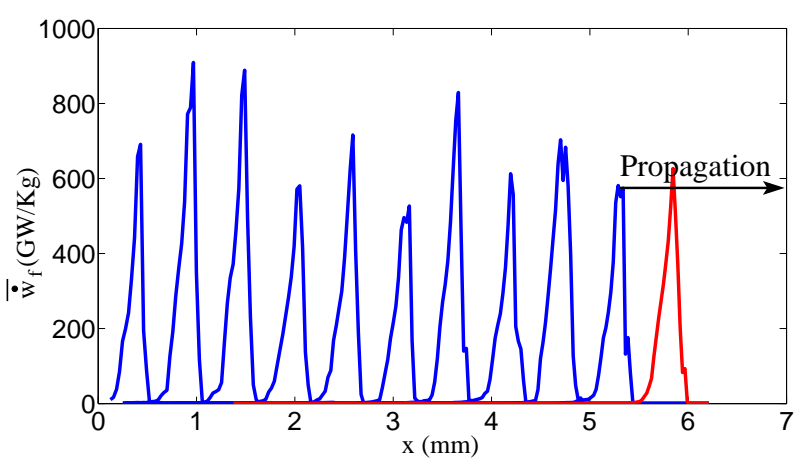

(b)

Figure 6.24: Predictions for the temporal variation of average (a) plastic heating rate $\overline{\dot{w}}_{p}$ and (b) friction heating rate $\overline{\dot{w}}_{f}$ for $v_{p}=50,250$ and $500 \mathrm{~m} / \mathrm{s}$. Profiles are shown in time-steps of $0.25 \mu \mathrm{s}$. 


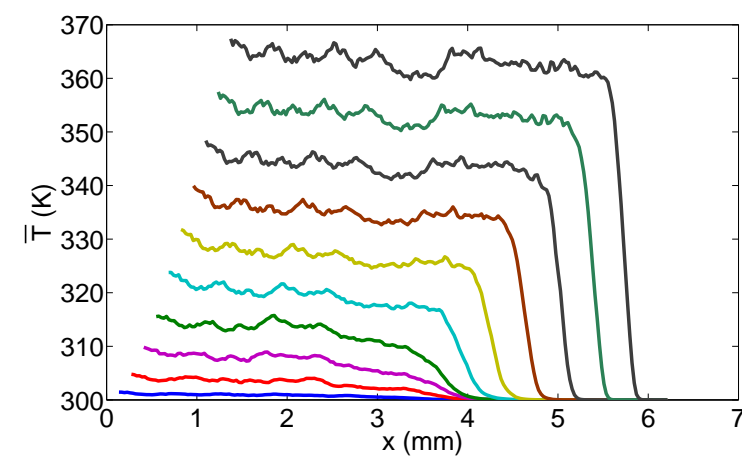

(a)

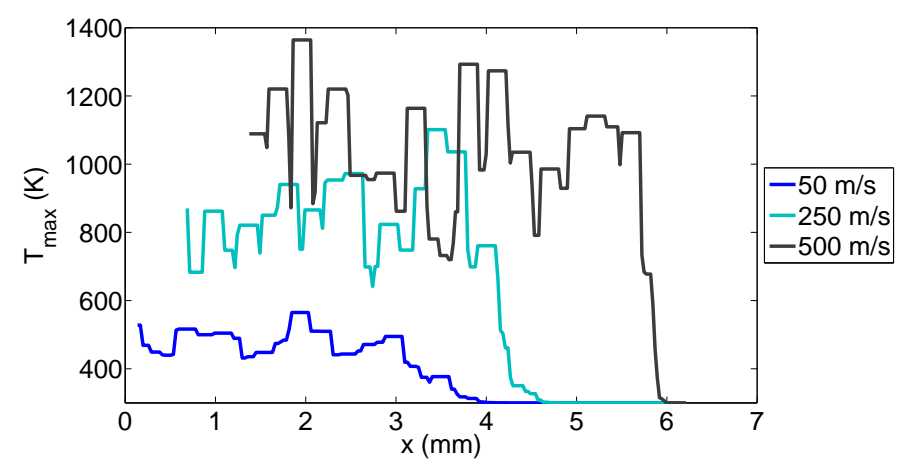

(b)

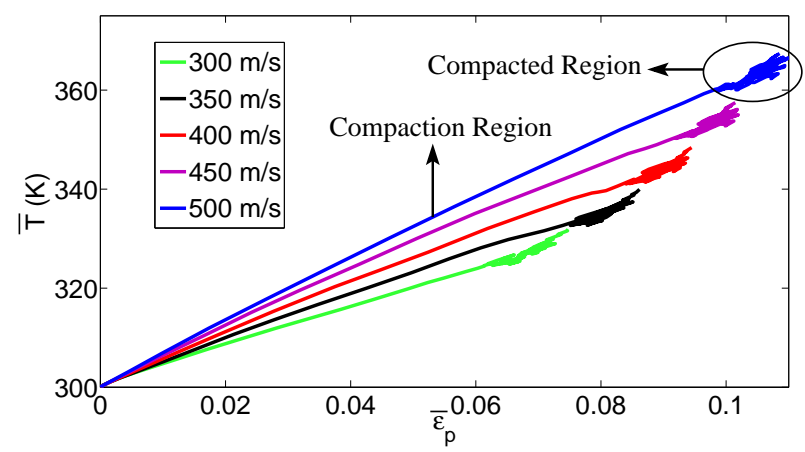

(c)

Figure 6.25: Predictions for the (a) average temperature $\bar{T}$ for $v_{p}=50-500 \mathrm{~m} / \mathrm{s}$, (b) maximum temperature $T_{\max }$ for $v_{p}=50,250$ and $500 \mathrm{~m} / \mathrm{s}$ and (c) variation of average temperature $\bar{T}$ with average effective plastic strain $\bar{\epsilon}_{p}$ at $2.75 \mu \mathrm{s}$.

increase to 1100 and $1400 \mathrm{~K}$ for $v_{p}=250$ and $500 \mathrm{~m} / \mathrm{s}$. This prediction highlights a key deficiency in current bulk models that are incapable of accurately predicting impact induced ignition of granular explosives based on average temperatures $[4,31]$ The accuracy of these models often hinges on ad hoc energy localization strategies whereby globally dissipated energy is deposited within small material volumes to estimate the effects of hot-spots. These strategies require knowledge of the underlying physics of energy dissipation mechanisms, such as plastic and friction work, occurring at the particle scale.

Spatial variations in the temperature field are best illustrated by particle scale contours. To this end, particle averaged temperature contours for the ensemble are shown in Fig. 6.26 for $v_{p}=50,250$, and $500 \mathrm{~m} / \mathrm{s}$ at $t=2.75 \mu \mathrm{s}$. Here, two key features are evident. First, the contours indicate that particles closest to the piston boundary experience the largest heating which is consistent with the predictions for the energetics already discussed. Second, the maximum 
particle averaged temperatures range from $312 \mathrm{~K}$ for $v_{p}=50 \mathrm{~m} / \mathrm{s}$ to $510 \mathrm{~K}$ for $500 \mathrm{~m} / \mathrm{s}$, which are lower than the ignition threshold for HMX. This prediction indicates that hot-spots relevant to combustion must occur at the sub-particle scale. To examine hot-spots at the sub-particle scale, two figures are presented. First, shown in Fig. 6.27 are finite element temperature contours for the case $v_{p}=500 \mathrm{~m} / \mathrm{s}$ at $t=2.75 \mu \mathrm{s}$. Here, local particle mass within the ensemble is heated to temperatures as high as $1400 \mathrm{~K}$, though these hot-spots are extremely small and difficult to observe in the plot. As such, the boxed region is magnified ten times to highlight this structure, where examples of heated mass are circled in the expanded figure. From the figure, it is evident that hotspots occur close to inter-particle contact interfaces primarily due to frictional dissipation. Second, shown in Fig. 6.28 are finite element adiabatic plastic temperature contours for $v_{p}=500 \mathrm{~m} / \mathrm{s}$ at $t=2.75 \mu \mathrm{s}$. Here, the adiabatic plastic temperature rise for each element is estimated by $\Delta T_{p}=W_{p} / m_{e} c_{v}$, where $W_{p}$ is the net plastic work associated with the element and $m_{e}$ is its mass. Local mass within the ensemble is heated to temperatures close to $750 \mathrm{~K}$, purely due to plastic work. Because this mass is also extremely localized, it is not evident in the plot. Plastically heated regions can be identified by magnifying a portion of the particle ensemble. From the expanded figure, it is clear that, unlike frictional hot-spots, those induced by plastic work occur in the interior of particles, close to contact surfaces. They are also substantially larger in size, and greater in number.

\section{- Hot-Spot Mass-Fraction}

The energy analysis performed in this study predicts maximum hot-spot temperatures ranging from 600 to $1400 \mathrm{~K}$ for piston speeds between 50 and $500 \mathrm{~m} / \mathrm{s}$. Whether these hot-spots are sufficient for prompt ignition, or they are quenched by thermal diffusion, depends on their thermal inertia (i.e., size and number density). Therefore, it is instructive to characterize the spatial and temporal variation of local hot-spot mass-fraction. To this end, hot-spot mass-fraction contours are plotted for which the vertical and horizontal axes give temperature rise and axial position, respectively; superimposed on these contours are the corresponding profiles of $\overline{\dot{w}}_{p}$ whose magni- 

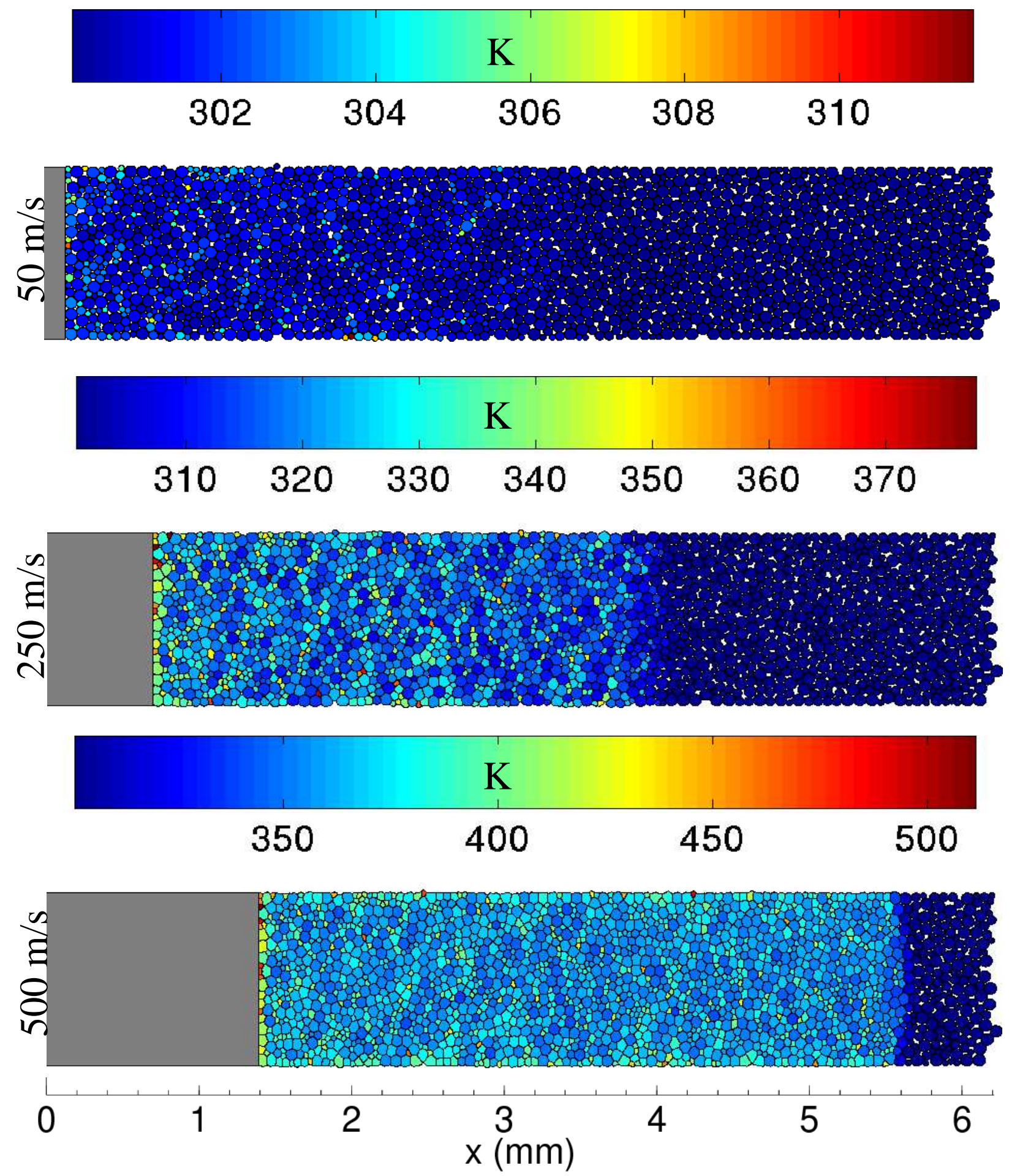

Figure 6.26: Predicted particle averaged temperature contours at $2.75 \mu \mathrm{s}$ for $v_{p}=50,250$ and $500 \mathrm{~m} / \mathrm{s}$. All values shown in the color-bar are in Kelvin. 


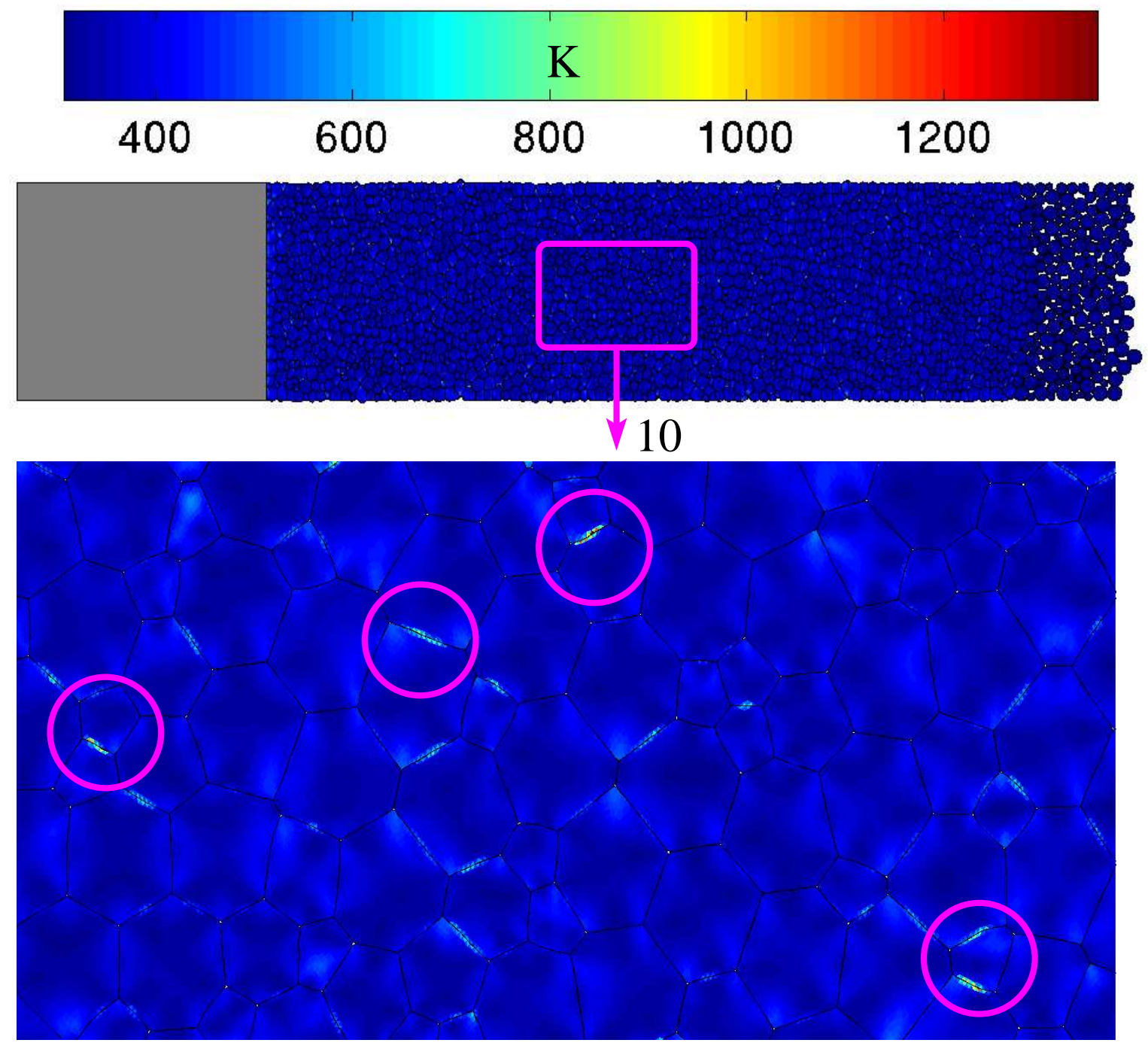

Figure 6.27: Predicted finite element temperature contour at $2.75 \mu \mathrm{s}$ for $v_{p}=500 \mathrm{~m} / \mathrm{s}$. All values shown in the color-bar are in Kelvin. 

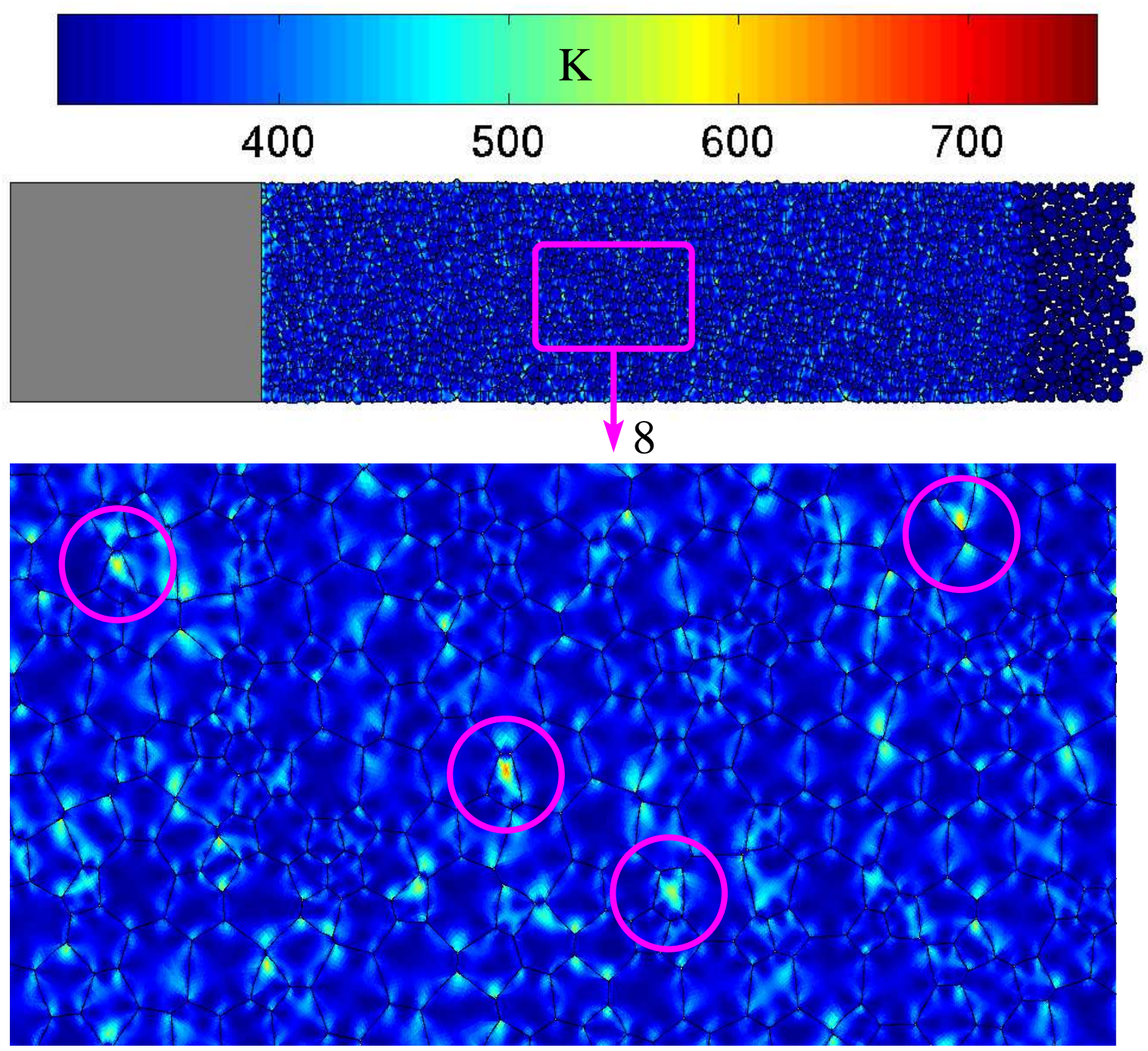

Figure 6.28: Predicted finite element adiabatic plastic temperature contour at $2.75 \mu \mathrm{s}$ for $v_{p}=$ $500 \mathrm{~m} / \mathrm{s}$. All values shown in the color-bar are in Kelvin. 
tudes are normalized for convenience. These profiles are intended to help correlate hot-spot mass fraction with wave structure.

Figure 6.29 shows the spatial and temporal variation in hot-spot mass fraction through the compaction zone for $v_{p}=50 \mathrm{~m} / \mathrm{s}$; corresponding plots are shown in Figs. 6.30 and 6.31 for $v_{p}=250$ and $500 \mathrm{~m} / \mathrm{s}$. The left boundary in these figures again represents the piston location, and the right boundary represents the free surface of the particle ensemble. The discrete appearance of these contours is a result of using a finite number of discrete intervals to represent temperature rise. The hot-spot contours are limited by the smallest mass-fraction $\left(\approx 10^{-5}\right)$ that can be resolved by the simulations, which is given by the mass of the smallest finite element within an averaging area divided by the total mass contained in that area.

As the compaction wave propagates through the domain, plastic and frictional heating induces temperature rises in particles within the compaction zone. Ahead of the plastic heating region, particles experience low heating due to the precursor which results in $\Delta T \leq 5 \mathrm{~K}$ for all piston speeds. For fixed time, heated mass steadily increases with distance behind the wave front within the compaction zone. Most mass is locally heated through a fixed temperature rise $\Delta T_{b}$, the value of which increases with piston speed. This region corresponds to a heated band of thickness $\Delta T_{b}$ near the bottom of each plot. Here, $\Delta T_{b} \approx 30,100$, and $200 \mathrm{~K}$ for $v_{p}=50,250$, and $500 \mathrm{~m} / \mathrm{s}$, respectively. The local mass-fraction that experiences a temperature rise between $5 \mathrm{~K}$ and $\Delta T_{b}$ varies between $10^{-1}$ and $10^{-3}$, respectively. For all piston speeds, a second heating band can be identified on the contours which contains mass-fractions ranging between $10^{-3}$ to $10^{-4}$, with temperature rises between $\Delta T_{b}$ and $\Delta T_{f}$, where $\Delta T_{f} \approx 70,200$, and $400 \mathrm{~K}$ for $v_{p}=50,250$, and $500 \mathrm{~m} / \mathrm{s}$, respectively. Also, the contours indicate that, for all piston speeds, small massfractions $\left(m=10^{-5}\right)$ experience $\Delta T \geq \Delta T_{f}$, up to a maximum temperature rise of 280,800 , and $1100 \mathrm{~K}$ for $v_{p}=50,250$, and $500 \mathrm{~m} / \mathrm{s}$, respectively. These hot-spots vary sporadically with axial position due to heterogeneities in the meso-structure; also, they increase in number with piston speed. Once created, these hot-spots maintain their respective temperatures for the duration of the simulation, indicating that thermal conduction has negligible influence over these fast time 


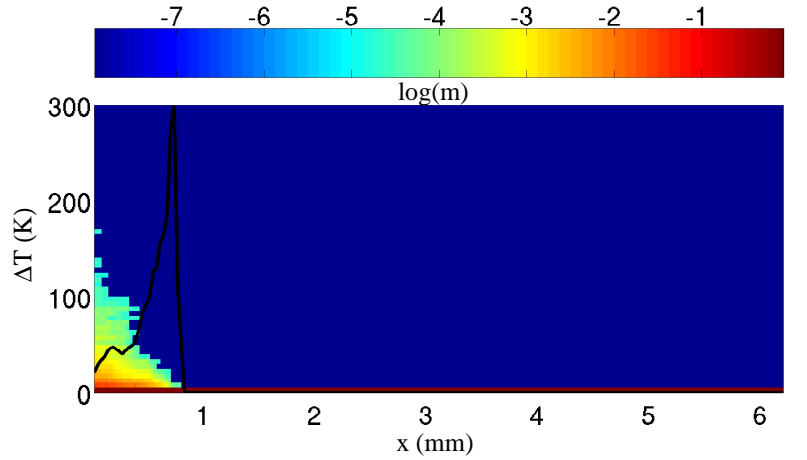

$0.5 \mu \mathrm{s}$

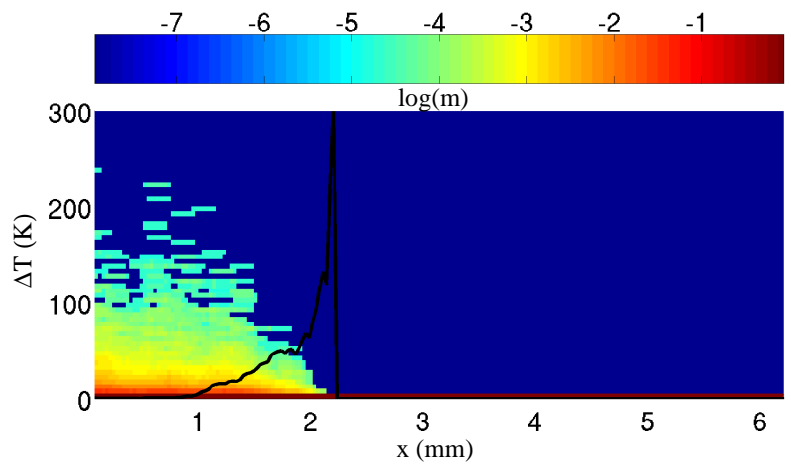

$1.5 \mu \mathrm{s}$

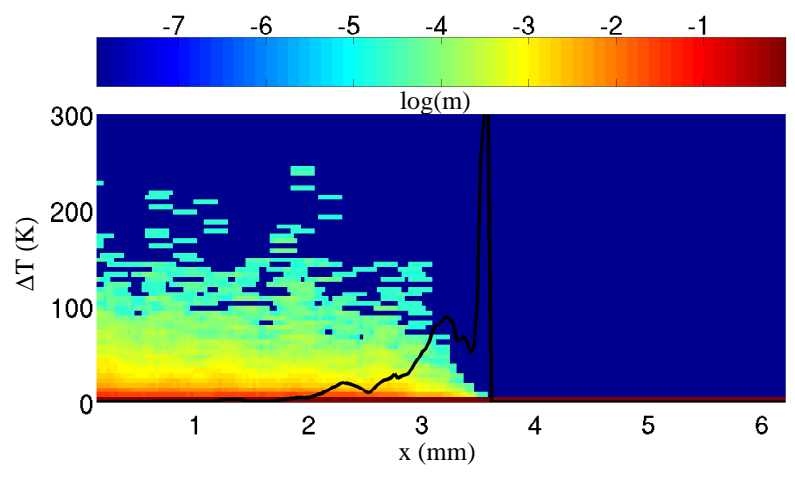

$2.5 \mu \mathrm{s}$
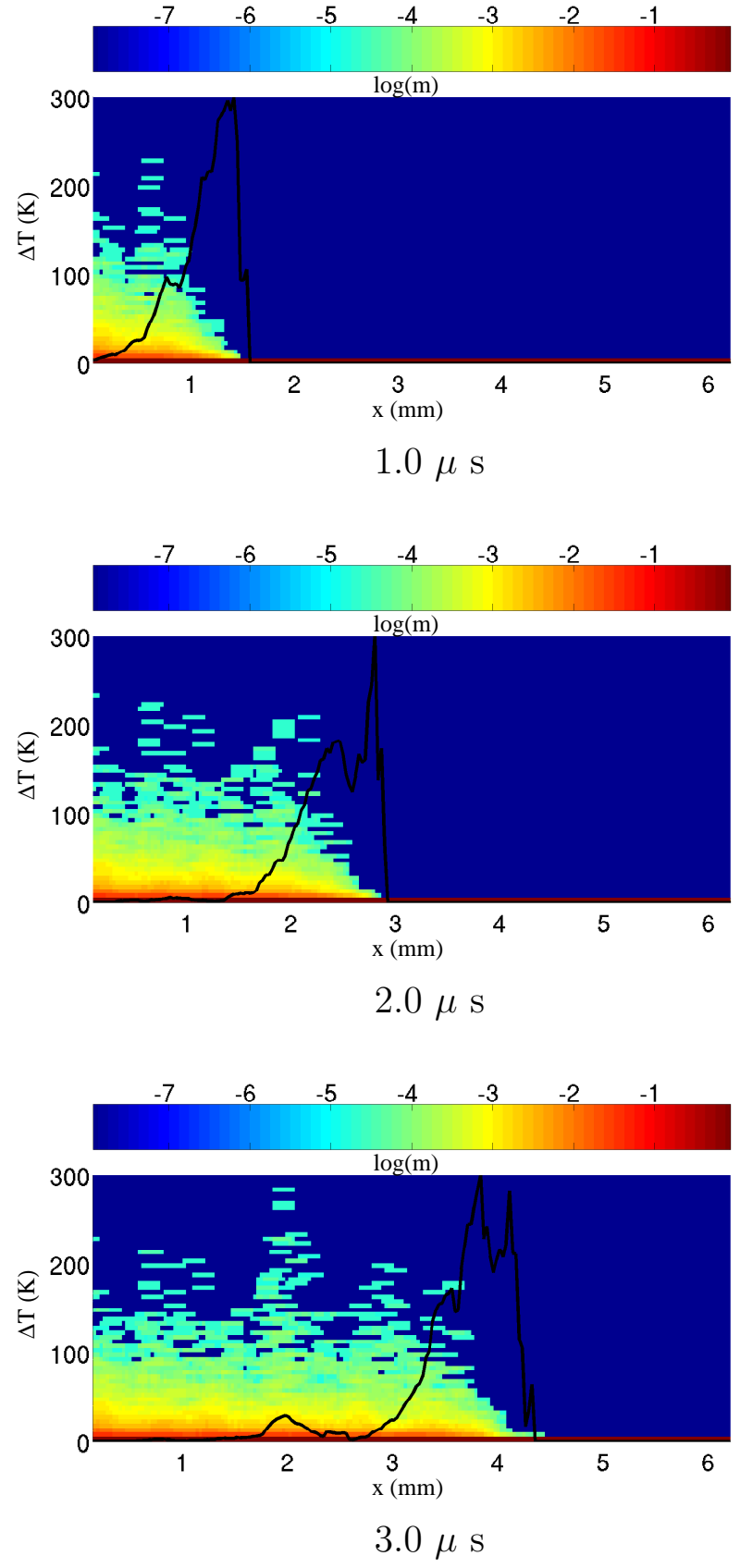

Figure 6.29: Predicted temporal variation of hot-spot mass-fraction for $v_{p}=50 \mathrm{~m} / \mathrm{s}$. Values in the color-bar represent the logarithm of mass-fraction. 


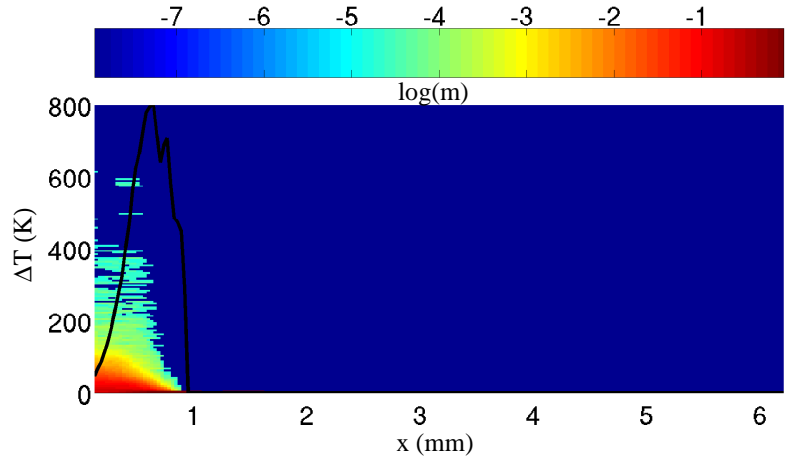

$0.5 \mu \mathrm{s}$

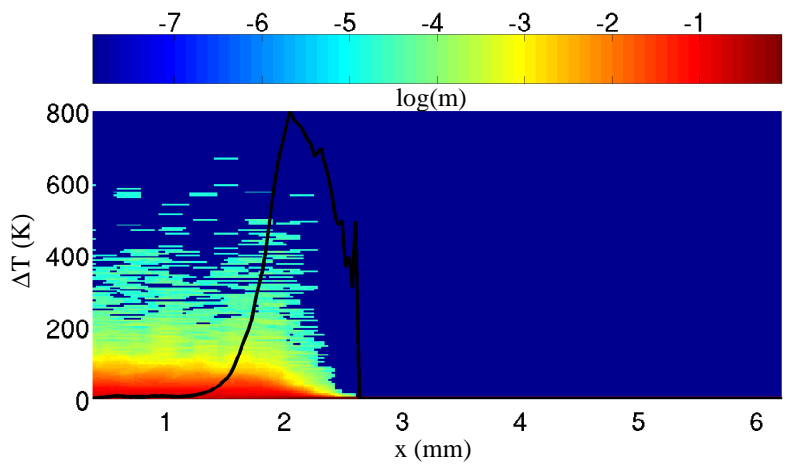

$1.5 \mu \mathrm{s}$

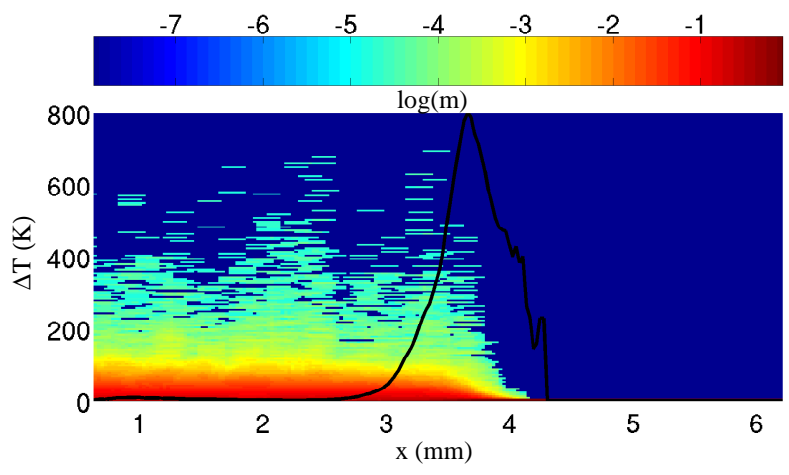

$2.5 \mu \mathrm{s}$
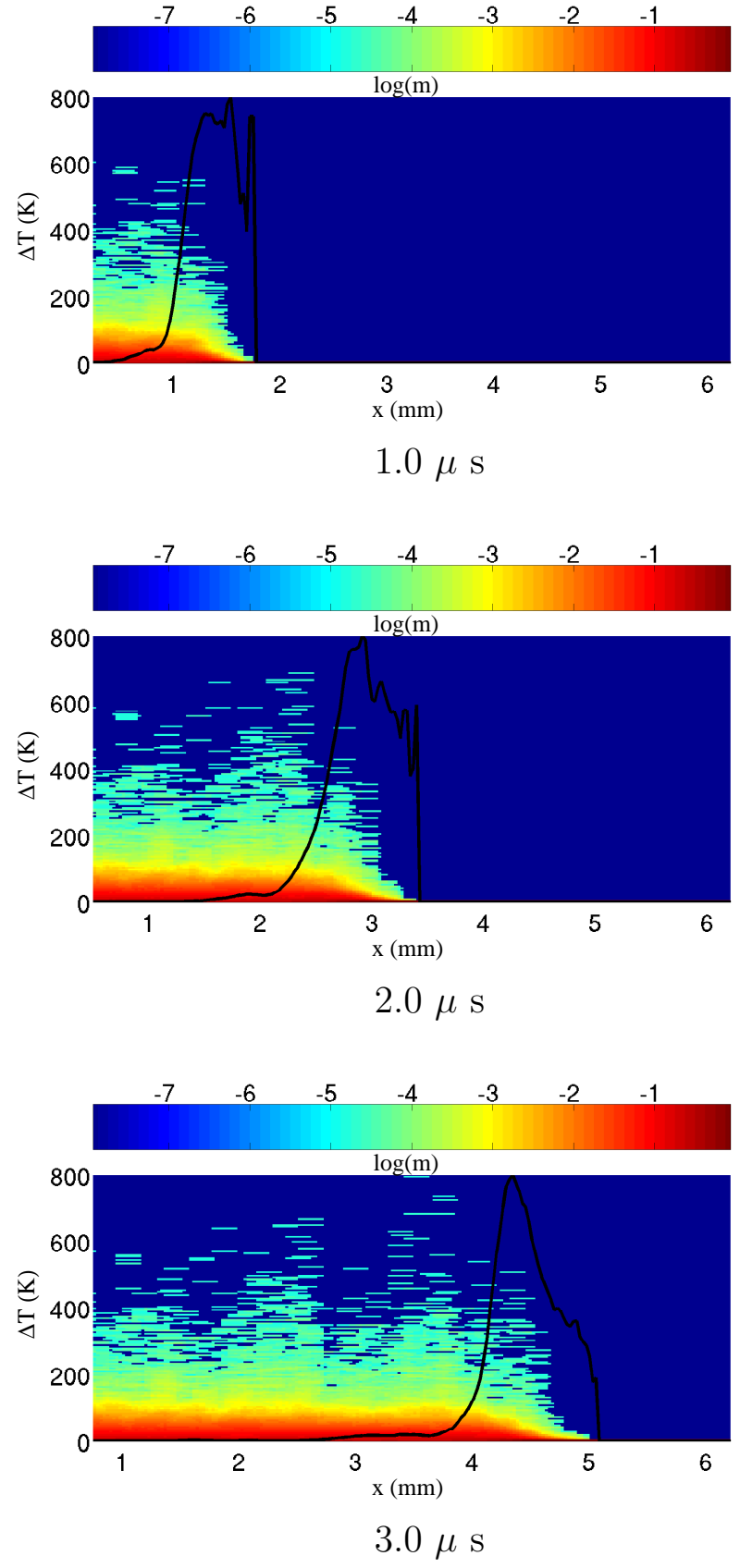

Figure 6.30: Predicted temporal variation of hot-spot mass-fraction for $v_{p}=250 \mathrm{~m} / \mathrm{s}$. Values in the color-bar represent the logarithm of mass-fraction. 


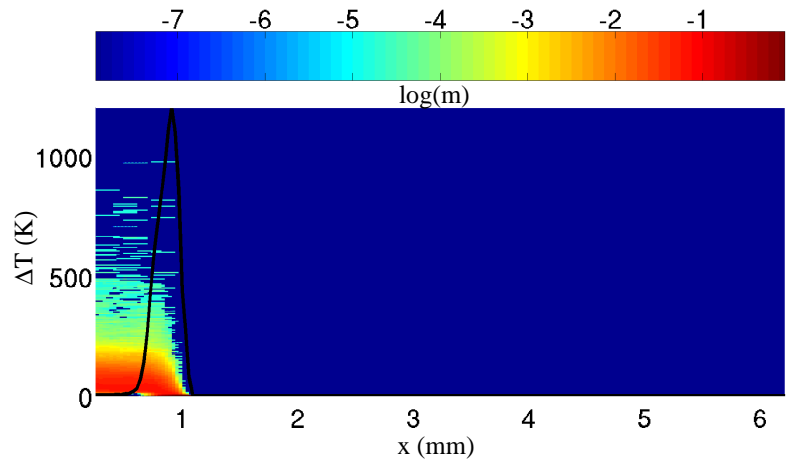

$0.5 \mu \mathrm{s}$

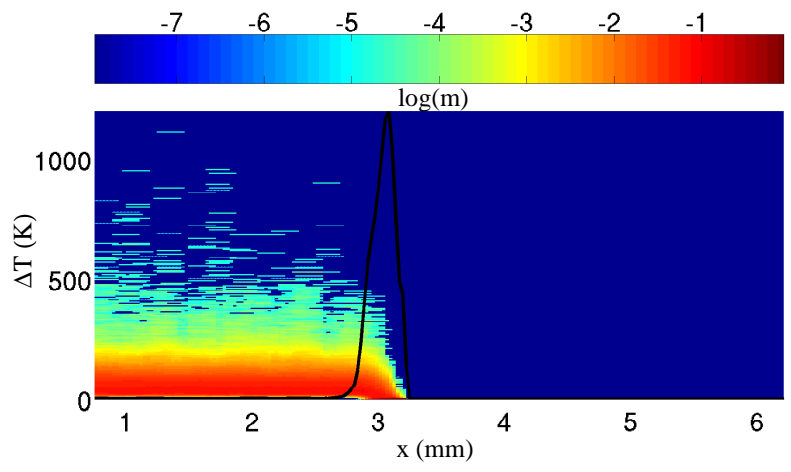

$1.5 \mu \mathrm{s}$

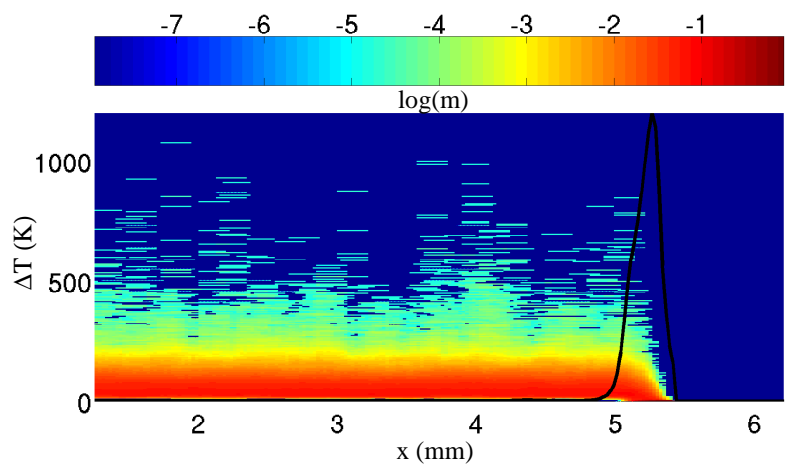

$2.5 \mu \mathrm{s}$
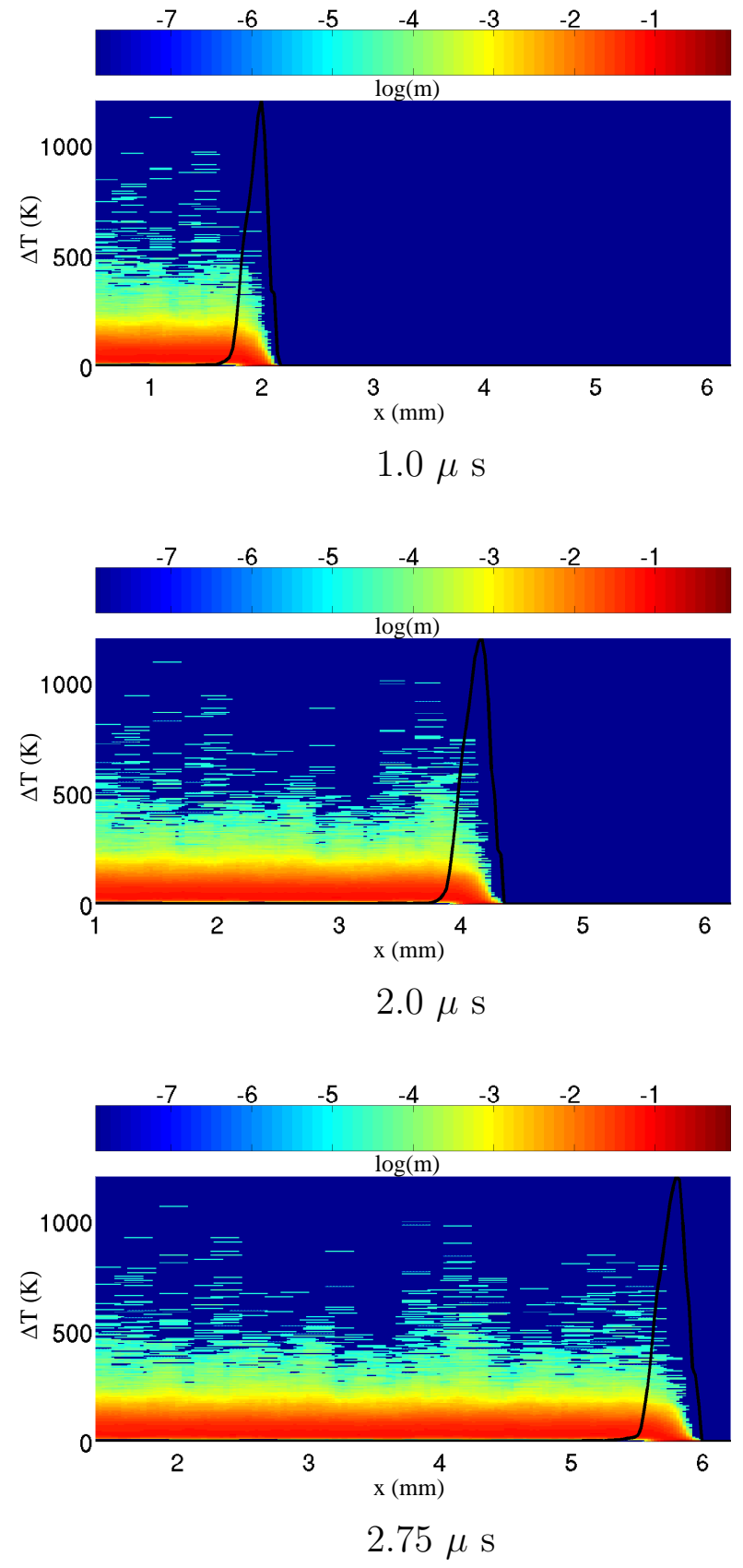

Figure 6.31: Predicted temporal variation of hot-spot mass-fraction for $v_{p}=500 \mathrm{~m} / \mathrm{s}$. Values in the color-bar represent the logarithm of mass-fraction. 
scales for the computational grid used in this study. Also, the hot-spot distribution behind the compaction wave exhibits negligible variation with time suggesting that frictional rearrangement due internal wave reflections within the compacted region is an insignificant heating mechanism.

The relative importance of friction work and plastic work in producing hot-spots can be examined by comparing hot-spot contours to those obtained based on an adiabatic plasticity theory. To this end, first, the adiabatic plastic temperature rise for each finite element $\Delta^{(j)}$ is computed as $\Delta \hat{T}_{p}=\hat{W}_{p} /\left(\rho A c_{v}\right)$, where $\rho$ is its current density and $\hat{W}_{p}$ is the net plastic work associated with $\Delta^{(j)}$ estimated by numerically integrating Eq. (2.50) over its current area $A$. Following this procedure for all finite elements describes an adiabatic temperature rise field, where thermal conduction effects and frictional effects are ignored, which can then be used to estimate the hot-spot contours in the standard way. Figure 6.32(a) gives hot-spot contours based on the predicted temperature field for $v_{p}=50,250$, and $500 \mathrm{~m} / \mathrm{s}$ at $t=2.75 \mu \mathrm{s}$. Corresponding hot-spot contours based on the adiabatic plastically induced temperature field are shown in Fig. 6.32(b). Predictions for $v_{p}=50 \mathrm{~m} / \mathrm{s}$ indicate that little plastic heating occurs for low speed impact. For this case, most of the mass affected by plasticity experiences $\Delta T \leq 25 \mathrm{~K}$. However, small, highly isolated mass-fractions $\left(m=10^{-5}\right)$ within the domain do experience substantial plastic heating leading to temperature rises as high as $150 \mathrm{~K}$. Comparing these predictions to the corresponding hot-spot contour shown in Fig. 6.32(a) indicates that almost all of the mass heated above $25 \mathrm{~K}$ is entirely due to frictional dissipation. Clearly, friction work is the dominant heating mechanism at lower speeds. For $v_{p}=250$ and $500 \mathrm{~m} / \mathrm{s}$, approximately $99.9 \%$ of the local mass experiences adiabatic plastic temperature rises of $\Delta T \leq \Delta T_{b}$. Thus, almost all mass heated to $\Delta T \geq 300 \mathrm{~K}$ is due to a combination of plasticity and friction, or friction alone. This observation indicates that, even for high piston speeds, friction plays a significant role in producing hot-spots.

Figure 6.33(a) shows predicted profiles for the average plastic and frictional heating rates corresponding to $v_{p}=50,250$, and $500 \mathrm{~m} / \mathrm{s}$ at $t=2.75 \mu \mathrm{s}$. Hot-spot mass-fractions are analyzed at fixed axial locations within and behind the compaction zone, denoted by (a), (b), and (c) in the figure; corresponding predictions are shown in Fig. 6.33(b). Here, temperature rise is plotted on 
$50 \mathrm{~m} / \mathrm{s}$
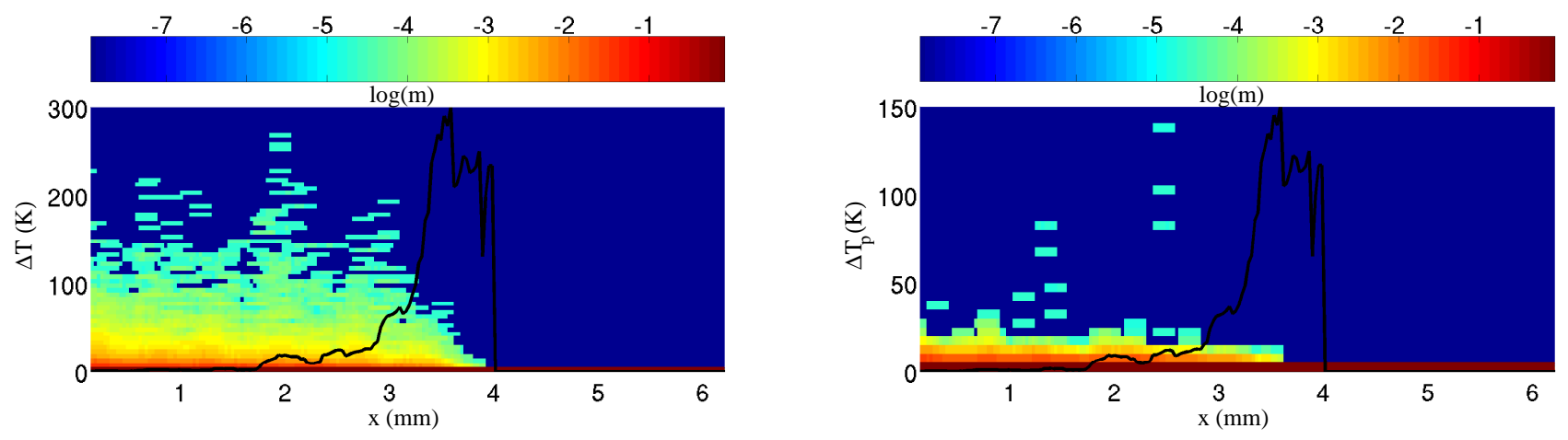

$250 \mathrm{~m} / \mathrm{s}$
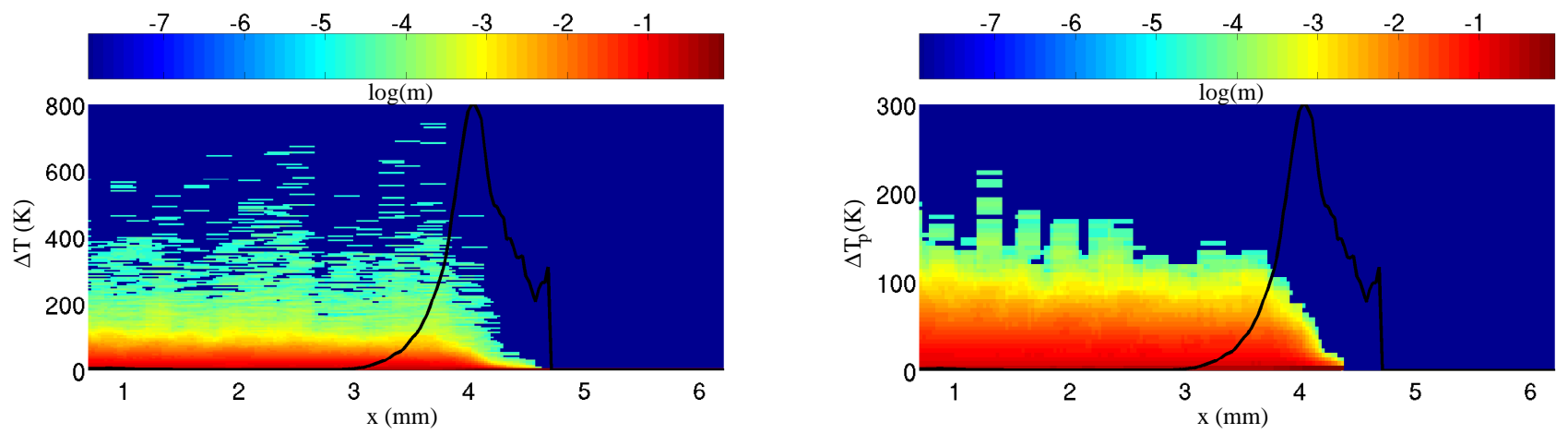

$500 \mathrm{~m} / \mathrm{s}$

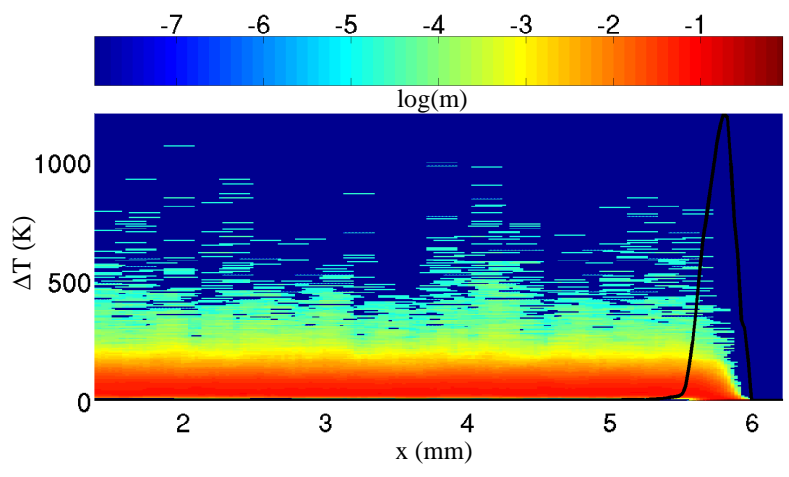

(a)

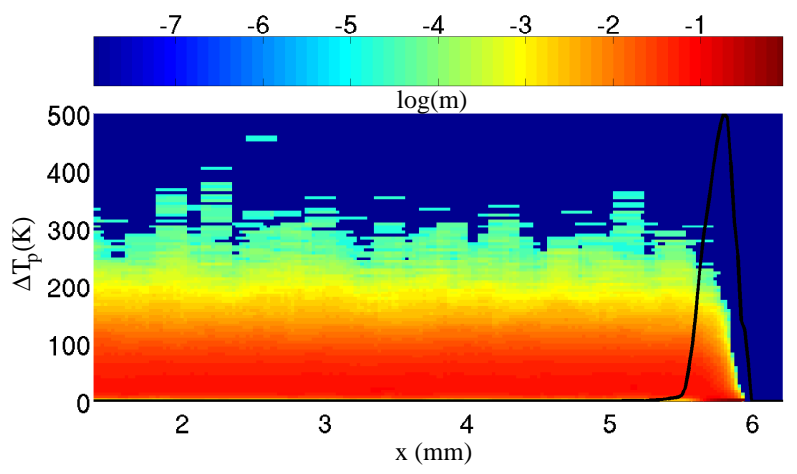

(b)

Figure 6.32: Predicted hot-spot mass-fraction contours based on temperature rise estimated using (a) energy analysis and (b) adiabatic plasticity theory at $2.75 \mu \mathrm{s}$ for $v_{p}=50,250$ and $500 \mathrm{~m} / \mathrm{s}$. Values in the color-bar represent the logarithm of mass-fraction. 
the horizontal-axis, and the logarithm of mass-fraction is plotted on the vertical-axis. A few key features are noteworthy. First, minimal heating occurs within the precursor. For $v_{p}=250 \mathrm{~m} / \mathrm{s}$, the hot-spot profile at location (a) within the precursor region indicates that approximately $99.5 \%$ of the local mass is unheated. At this location, maximum temperature rises of only $30 \mathrm{~K}$ are induced corresponding to mass-fractions of approximately $10^{-4.2}$. Second, the mass-fraction that experiences a fixed temperature rise $\Delta T$ increases through the compaction zone. For example, for $v_{p}=50 \mathrm{~m} / \mathrm{s}$, the fraction of mass heated to $\Delta T=10 \mathrm{~K}$ increases from $10^{-3.2}$ at location (a) to $10^{-2.2}$ at location (b). For $250 \mathrm{~m} / \mathrm{s}$, the fraction of mass heated to $\Delta T=100 \mathrm{~K}$ increases from $10^{-4}$ at location (b) to $10^{-2.9}$ at location (c). Third, temperatures significantly increase from the front to the rear of the compaction zone. For example, for $v_{p}=50 \mathrm{~m} / \mathrm{s}$, the maximum temperature rise at location (a), which corresponds to the location of maximum average plastic heating rate, is approximately $75 \mathrm{~K}$; the mass-fraction associated with this temperature rise is approximately $10^{-5}$. Similar mass-fractions experience a maximum temperature rise of $\Delta T \approx$ $135 \mathrm{~K}$ at location (c) behind the compaction zone. Fourth, the average behavior of hot-spot distributions exhibits only marginal differences with axial position behind the compaction zone. For example, for $v_{p}=500 \mathrm{~m} / \mathrm{s}$, the hot-spot mass-fractions at locations (b) and (c) behind the wave are similar. This behavior is also predicted at locations (b) and (c) for the $50 \mathrm{~m} / \mathrm{s}$ case. However, the high temperature end of these distributions, which is dominated by friction work, exhibits large amplitude, high frequency fluctuations. These fluctuations, which may be important for producing reactive hot-spots, are attributable to highly localized, complex stick-slip friction behavior at contact interfaces and numerical resolution limitations. Predicted peak temperatures are physically limited by thermal conduction which is difficult to numerically resolve for high speed impact without the use of excessively (and perhaps prohibitively) fine grids. As such, sub-grid models are likely needed to accurately describe the effect of thermal conduction on the reactive tribology of granular energetic solids.

As mentioned, the chemical reaction rate of HMX is highly sensitive to temperature. Important ignition characteristics such as run distance to detonation and time to ignition are determined 
$50 \mathrm{~m} / \mathrm{s}$
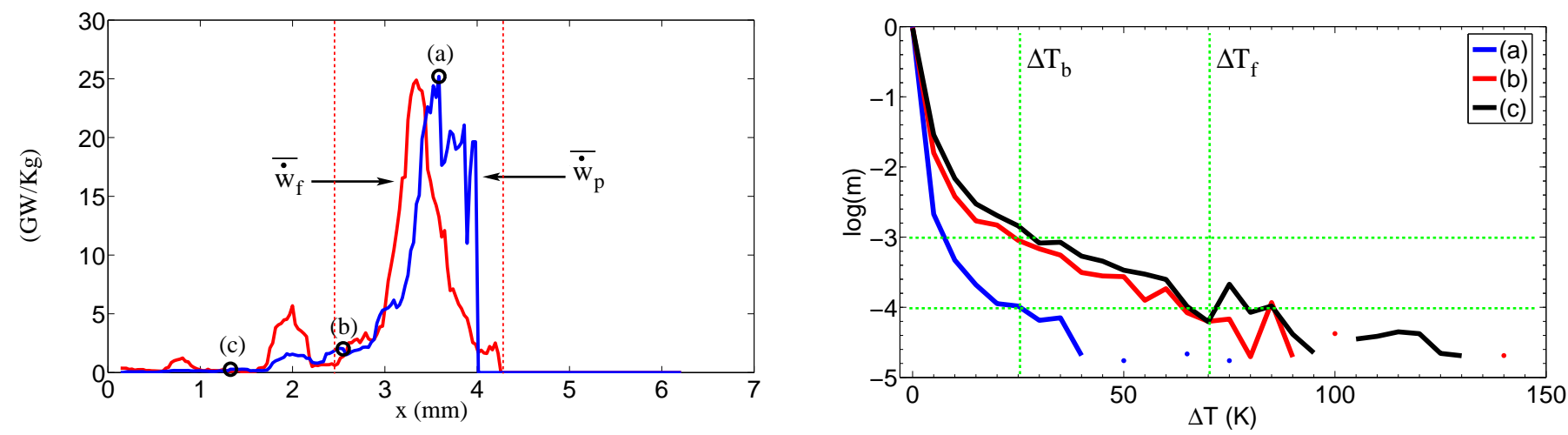

$250 \mathrm{~m} / \mathrm{s}$
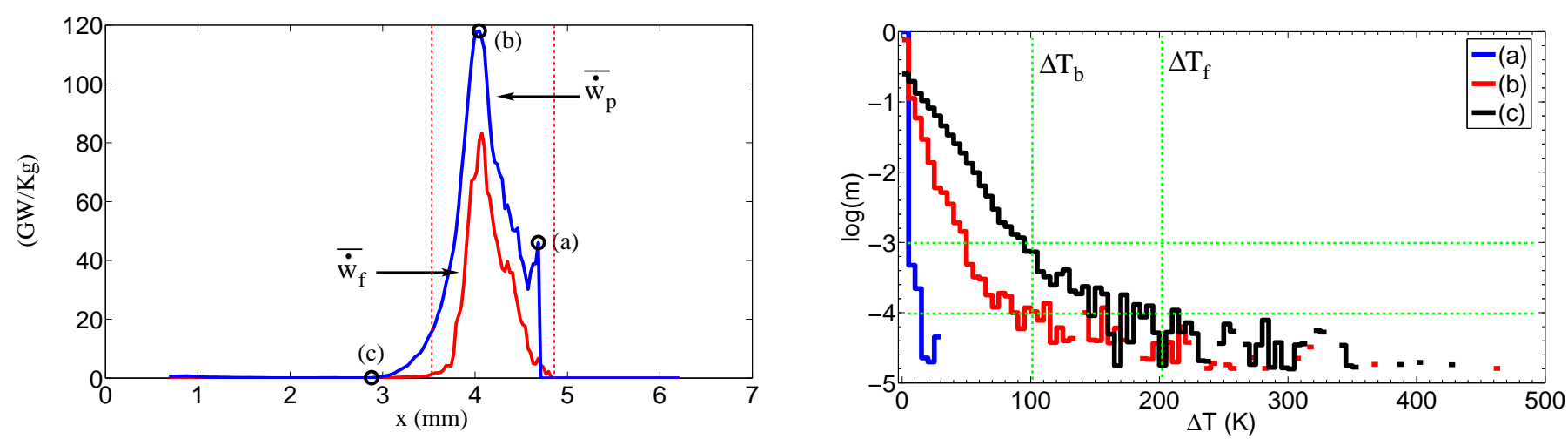

$500 \mathrm{~m} / \mathrm{s}$
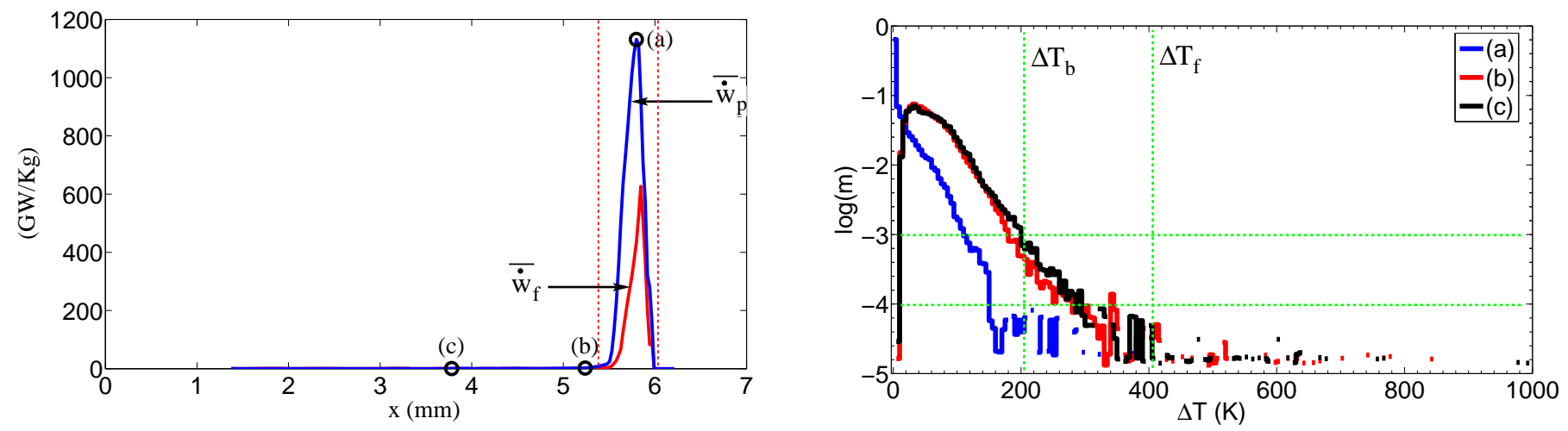

Figure 6.33: Predicted spatial variation of hot-spot mass-fraction through the compaction wave at $2.75 \mu \mathrm{s}$ for $v_{p}=50,250$ and $500 \mathrm{~m} / \mathrm{s}$. 


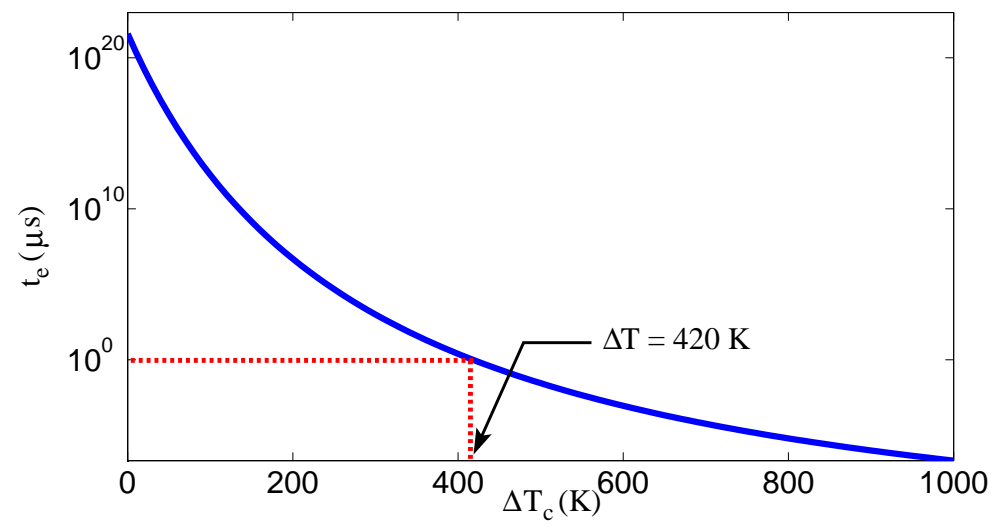

Figure 6.34: Variation of time to thermal explosion with hot-spot temperature.

by particle scale chemical kinetics and depend on factors such a hot-spot size, temperature and number density. Therefore, a complete description of impact induced ignition should account for combustion chemistry. An asymptotic theory for the two-phase (solid-gas) thermal explosion of HMX performed by Gonthier and Jogi [50] gives the following relation between the thermal explosion time $t_{e}$ and hot-spot temperature $T_{c}$ :

$$
t_{e}=\frac{c_{v} T_{c}^{2}}{Z T^{*}\left(q_{h}+\left(c_{v}-c_{v g}\right) T_{c}\right)} \exp \left(\frac{T^{*}}{T_{c}}\right)
$$

where $c_{v g}=1 \mathrm{KJ} /(\mathrm{Kg}-\mathrm{K})$ is the specific heat of the gas, $Z=5 \times 10^{19} s^{-1}$ is the pre-exponential factor, $T^{*}=2.65 \times 10^{4} \mathrm{~K}$ is the activation temperature, and $q_{h}=5.84 \times 10^{6} \mathrm{~J} / \mathrm{Kg}$ is the specific heat of combustion. Figure 6.34 shows the variation of $t_{e}$ with $\Delta T_{c}$. This theory suggests, for simulation time scales in this study $(\approx 1 \mu \mathrm{s})$, that predicted hot-spot temperature rises of $\Delta T \geq 420 \mathrm{~K}$ will likely trigger combustion. A more comprehensive thermal explosion analysis of the critical conditions for impact and shock induced hot-spots in HMX required for initiation and sustained combustion was performed by Tarver et.al. [126]. Their analysis included a detailed description of the multi-step chemical kinetic decomposition models and predicted that cylindrical hot-spots with diameter $2.6248 \mu \mathrm{m}$, which corresponds to the average finite element size used in this study, required critical temperatures close to $1000 \mathrm{~K}$ for combustion initiation. However, a more rigorous description of particle scale chemical kinetics is needed to better predict combustion thresholds. 
Typical energetic particle ensembles used in practice vary in size from 0.01-1 $\mathrm{m}$ and are composed of millions of particles of different sizes and shapes. Performing meso-scale computations over these engineering lengths is impossible. Though bulk models can be feasily applied to large domains, they only describe the material's thermomechanical response in terms of average quantities that are largely insensitive to meso-scale features important for combustion initiation. In the absence of meso-scale data, the onus is on meso-scale models, such as the one used in this study, to provide statistically meaningful hot-spot information that can be efficiently incorporated into bulk models to improve their predictability of impact induced combustion. To this end, local hot-spot mass-fraction distributions may be used to construct improved, hot-spot motivated bulk burn models. One possible approach is to fit predicted hot-spot mass-fraction curves with standard distribution functions, and to correlate these functions with average volumetric dissipation rate, which is easily predicted by bulk models, and meso-structure, possibly taking advantage of similitude if it exists. Because much of the hot-spot mass is induced by plastic work, whereas only a very small amount of mass, corresponding to the high temperature end of the distribution curve, is induced by friction work, separate distribution functions may be required to collectively characterize their effects. For example, the bulk response of the hot-spot mass-fraction due to plastic work may be approximated by a gamma probability density function, which is parameterized by a shape factor $A$ and a scale factor $B$ :

$$
\xi(\chi ; A, B)=\chi^{A-1} \frac{\exp (-\chi / B)}{B^{A} \Gamma(A)} .
$$

The factors $A$ and $B$ will generally depend on the average volumetric dissipation rate and the material meso-structure. Gamma distributions for several values of $A$ and $B$ are shown in Fig. $6.35(\mathrm{a})$

Modeling the small mass, high temperature end of the distribution curve may be more difficult because of its high sensitivity to thermal conduction and computational resolution. Insight may be obtained from the analytical solution for time-dependent thermal transport within a semi-infinite 


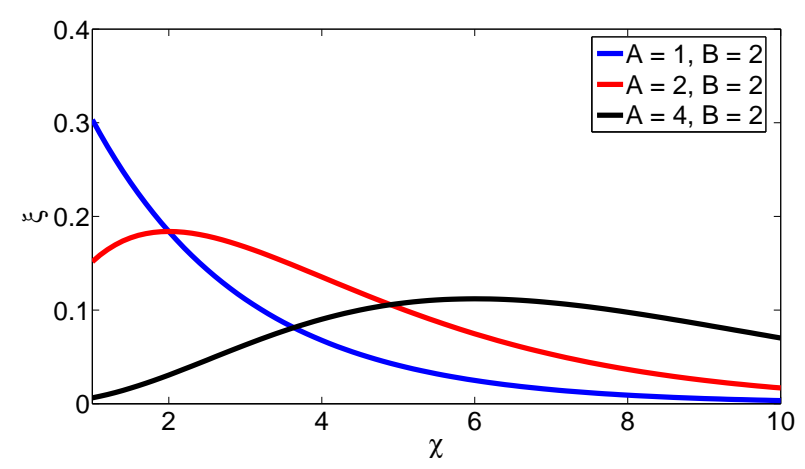

(a)

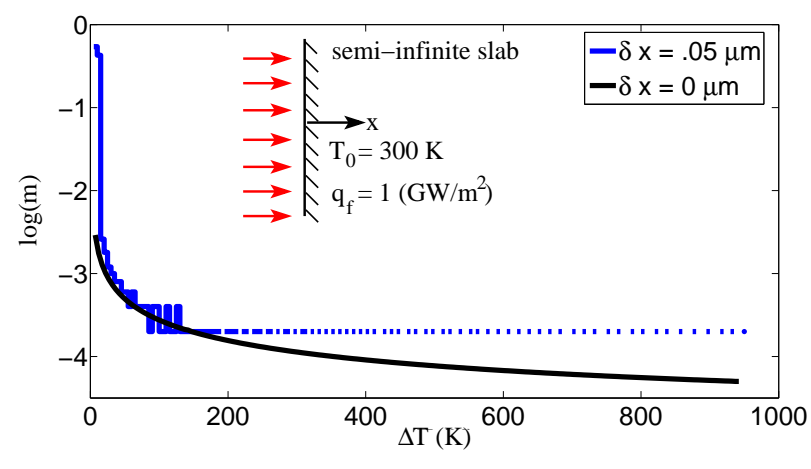

(b)

Figure 6.35: (a) Gamma distribution function. (b) Hot-spot distribution for semi-infinite slab heat transfer problem.

slab having a constant applied heat flux boundary condition and a uniform initial temperature. The analytical solution to this problem at time $t=\tau$ is given by:

$$
T(x)=T_{0}+\frac{2 q_{f} \sqrt{\alpha_{t} \tau / \pi}}{k_{T}} \exp \left(\frac{-x^{2}}{4 \alpha_{t} \tau}\right)-\frac{q_{f} x}{k_{T}}\left(1-\operatorname{erf}\left(\frac{x}{2 \sqrt{\alpha_{t} \tau}}\right)\right),
$$

where $T_{0}$ is the initial temperature, $q_{f}$ is the applied heat flux, and $\alpha_{t}$ is the thermal diffusivity. Because thermal conduction effects are insignificant over the short particle residence times within the wave $(\leq 1 \mu \mathrm{s})$, based on the computational grids used in this study, each frictional contact interface may be locally approximated as an infinite slab. To illustrate how numerical resolution can affect the high temperature end of the distribution curve, assuming that the frictional heat flux and material density are constant over a representative time $\tau=1 \mu \mathrm{s}$, the $x$-axis is discretized into line elements of width $\delta x$ centered about a prescribed number of nodal positions $x_{i}$, where the mass of each element is allocated to its node. Next, the temperature field is discretized into uniform increments of $\delta T \mathrm{~K}$ and nodal mass is sorted based on its temperature rise, which is estimated by Eq. (6.15). Finally, the hot-spot mass fraction is computed in the standard way by summing the mass within each temperature rise interval and dividing the result by the total mass. For values of $\delta x=0.5 \mu \mathrm{m}, \delta T=5 \mathrm{~K}$, and $q_{f}=1 \mathrm{GW} / \mathrm{m}^{2}$, the hot-spot mass-fraction distribution is shown in Fig. 6.35(b). Also shown in the figure is the analytical solution obtained by taking $\delta x \rightarrow 0$. These results illustrate that, based on an analytical solution for the temperature field, 
a discrete hot-spot distribution is obtained due to resolution limits. The discrete distribution resembles the analytical solution at the low temperature end, but deviates from the analytical solution at the high temperature end. The discrete hot-spot distribution shows similar qualitative and quantitative trends to distribution curves predicted in this study. 


\section{Chapter 7}

\section{Parameteric Sensitivity}

Predictions presented in the previous chapter highlight the relative importance of friction work and plastic work as hot-spot mechanisms and their sensitivity to piston speed. Additionally, these dissipation mechanisms may be sensitive to the choice of material properties such as friction coefficient $\mu$ and viscosity $\nu$, and to the assumed particle size distribution. Majority of meso-scale models found in the literature adopt the Eulerian framework, wherein, an accurate description of friction requires complex interface reconstruction algorithms that accurately preserve material interfaces. In the absence of such algorithms, researchers have ignored frictional effects and therefore little work has been done to characterize the friction coefficient of the materials under consideration. Furthermore, in meso-scale models that include a rate dependent constitutive theory to describe material motion, the viscosity parameter is generally obtained by correlating numerical predictions to limited experimental wave profile data. Uncertainty exists regarding the choice of this parameter and its influence on the system thermomechanics. In the absence of additional experimental data, meso-scale simulations can be used to investigate the influence of the friction coefficient and viscosity on the compaction wave structure. Predictions from these studies are presented in this chapter. Predictions for the influence of the friction coefficient and the viscosity parameter on the thermomechanical fields are presented in Section [7.1] and [7.2], respectively. Lastly, numerical predictions demonstrating the influence of particle size distribution on the compaction wave behavior is presented in Section [7.3].

\subsection{Friction Coefficient}

In this section, predictions are given that illustrate how friction coefficient $\mu$ affects the system thermomechanics. To this end, an alternate friction coefficient is chosen from the values $\mu=0.0$, 0.05 and 0.15 , and for each $\mu$ a set of 10 simulations are performed by varying the piston speed between $v_{p}=50 \mathrm{~m} / \mathrm{s}$ to $500 \mathrm{~m} / \mathrm{s}$, in $50 \mathrm{~m} / \mathrm{s}$ increments. For each simulation, all other material 


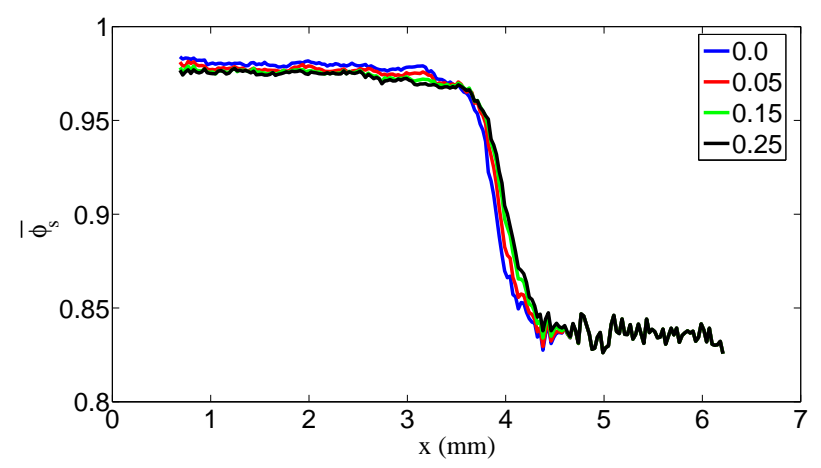

(a)

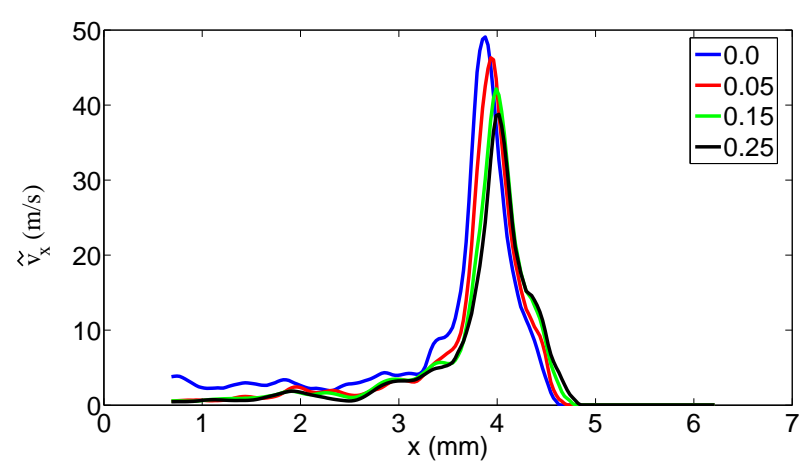

(b)

Figure 7.1: Predictions for the (a) average solid volume fraction $\bar{\phi}_{s}$ and (b) RMS fluctuation in axial velocity $\widetilde{v}_{x}$ at $2.75 \mu \mathrm{s}$ for $v_{p}=250 \mathrm{~m} / \mathrm{s}$ for $\mu=0.0,0.05,0.15$ and 0.25 .

and numerical parameters are fixed at the baseline values listed in Table 4.1. Wave profiles and contour plots given in this section correspond to time $t=2.75 \mu \mathrm{s}$ for the case $v_{p}=250 \mathrm{~m} / \mathrm{s}$, unless otherwise stated. The mechanical response is discussed first followed by the thermal response.

\subsubsection{Mechanical Response}

Figure 7.1(a) shows spatial profiles for the average solid volume fraction $\bar{\phi}_{s}$ for $\mu=0.0$, $0.05,0.15$ and 0.25 . The predictions indicate that while profiles of $\bar{\phi}_{s}$ display similar qualitative trends for all four cases, the quasi-steady final compacted value $\bar{\phi}_{s, f}$ decreases marginally with friction coefficient. Increasing $\mu$ increases the minimum tangential traction required for slip to occur. Subsequently, stick behavior becomes increasingly important at higher friction coefficients resulting in a frictionally rigid bed, whereas, a zero friction coefficient facilitates greater mobility and therefore greater compaction. This result is also observed in Fig. 7.1(b) which plots the RMS axial velocity fluctuation profiles for all the cases. From the figure, it is evident that the peak of the fluctuations decreases with decreasing friction coefficient indicating that paritcle mobility is inversely related to $\mu$. Although not shown here, the average axial velocity and pressure profiles for all the friction coefficients showed similar qualitative and quantitative trends indicating that the pressure field is not sensitive to the choice of $\mu$.

Predicted profiles of average Von Mises stress $\overline{\boldsymbol{\tau}}_{e}$ and the average effective plastic strain $\bar{\epsilon}_{p}$ for all the friction coefficients are shown in Fig. 7.2. For all the cases, $\overline{\boldsymbol{\tau}}_{e}$ relaxes to below the yield 


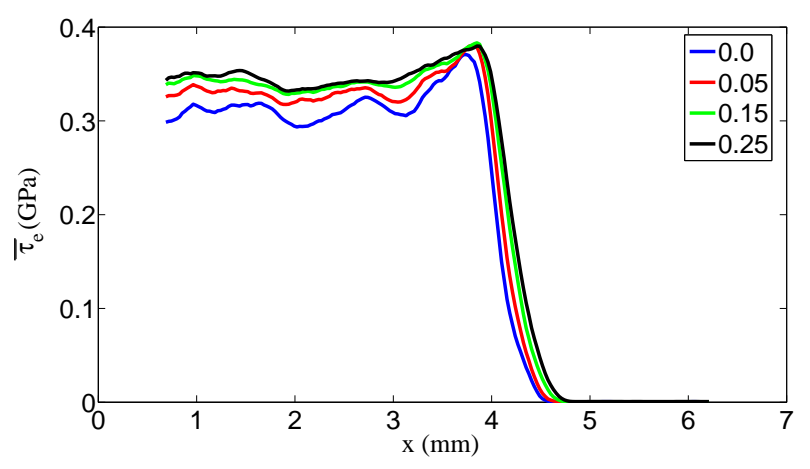

(a)

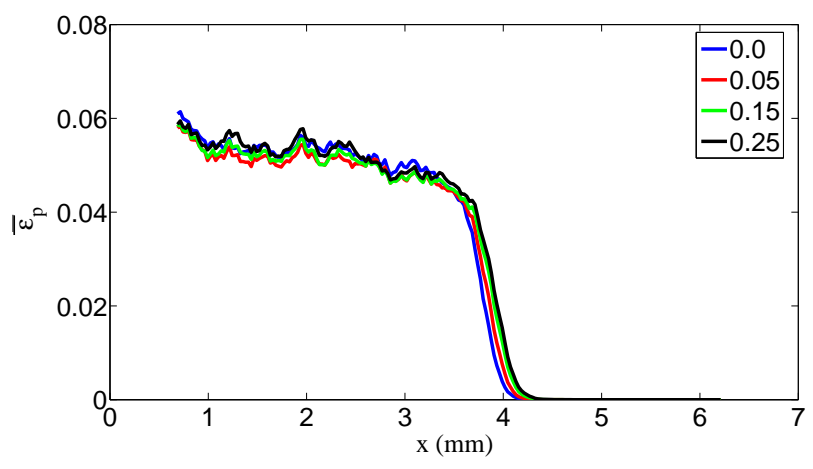

(b)

Figure 7.2: Predictions for the (a) average Von Mises stress $\overline{\boldsymbol{\tau}}_{e}$ and (b) average effective plastic strain $\bar{\epsilon}_{p}$ at $2.75 \mu \mathrm{s}$ for $v_{p}=250 \mathrm{~m} / \mathrm{s}$ for $\mu=0.0,0.05,0.15$ and 0.25 .

surface. However, increased mobility at lower $\mu$ facilitates greater relaxation of the deviatoric stresses and therefore $\overline{\boldsymbol{\tau}}_{e}$. For all the cases, profiles of $\bar{\epsilon}_{p}$ show similar qualitative and quantitative trends indicating that the friction coefficient has little influence on plastic deformation. Figure 7.3 plots the variation of the leading and trailing wave speeds with respect to piston speed for all the friction coefficients. From the figures, a few cursory observations are noteworthy. First, for all the friction coefficients, the precursor outruns the trailing wave at low piston speeds, and they coalesce at higher piston speeds $\left(v_{p} \geq 250 \mathrm{~m} / \mathrm{s}\right)$. Second, for a given piston speed, both the leading and trailing wave speed increase with increasing $\mu$. This is because a larger friction coefficient results in a frictionally rigid bed that aids wave propagation in the primary axial direction. Third, although the leading and trailing wave speeds are similar for all values of $\mu$ at high piston speeds ( $\geq 250 \mathrm{~m} / \mathrm{s}$ ), significant differences are observed at lower piston speeds. The plateau in the wave speed profiles becomes less prominent with decreasing friction coefficient as the profiles exhibit a linear dependence on $v_{p}$. These predictions indicate that while the wave speeds are insensitive to friction coefficient at high piston speeds, at low piston speeds, frictional stick promotes more efficient stress transmission between particles which results in faster wave speeds.

\subsubsection{Thermal Response}

To demonstrate the influence of $\mu$ on the thermal response of the particle ensemble, the temperature field is first considered. Figure 7.4(a) plots the average temperature profiles for all 


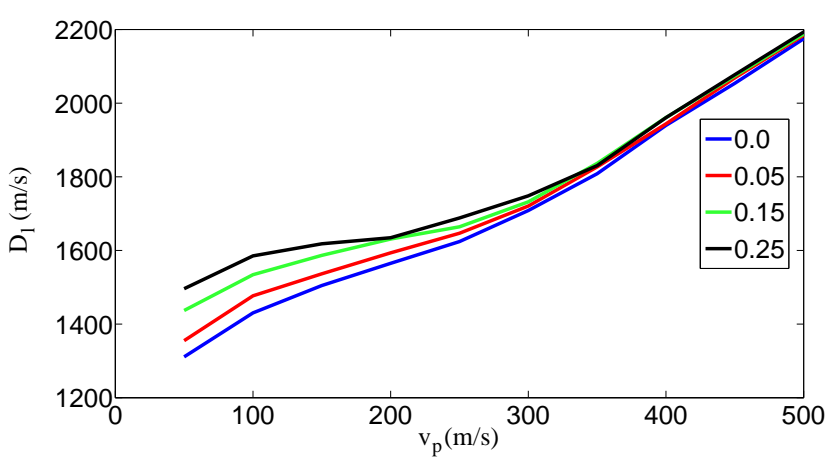

(a)

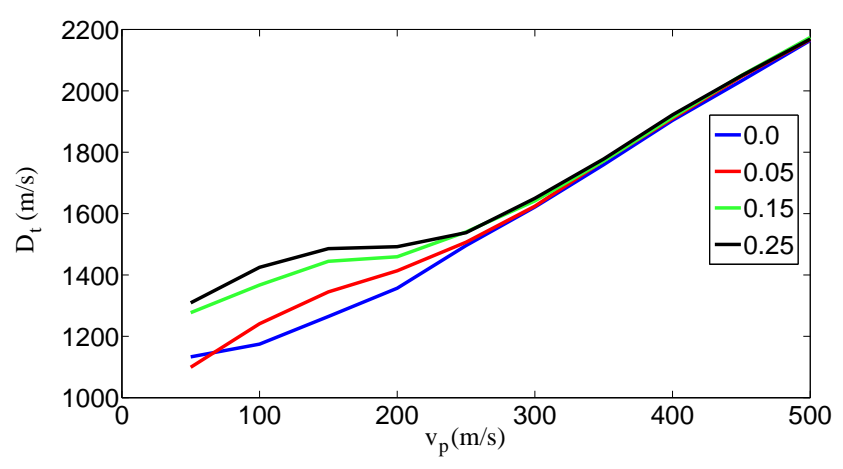

(b)

Figure 7.3: Predicted variation in (a) precursor $\left(D_{l}\right)$ and (b) plastic $\left(D_{t}\right)$ wave speed with piston speed $\left(v_{p}\right)$ for $\mu=0.0,0.05,0.15$ and 0.25 .

the friction coefficients for $v_{p}=250 \mathrm{~m} / \mathrm{s}$ at $2.75 \mu \mathrm{s}$. For all values of $\mu$, the average temperature profiles display similar qualitative trends with the quasi-steady bulk temperatures behind the compaction wave only increasing marginally with $\mu$. Because the average temperatures are largely representative of inelastic effects, these predictions indicate that plastic heating is insensitive to $\mu$. The influence of $\mu$ on the local temperature field is best demonstrated by considering the maximum temperature profiles, which are shown in Fig. 7.4(b). From the figure, it is evident that the maximum temperatures induced within the bed increase substantially with $\mu$. Clearly, while a maximum temperature of only $475 \mathrm{~K}$ is predicted for the frictionless case, temperatures close to $1100 \mathrm{k}$ are predicted for the baseline case. These predictions further highlight the importance of friction work as a hot-spot mechanism and the influence of the friction coefficient on hot-spot temperatures.

The influence of $\mu$ on the hot-spot mass-fraction contours is now considered. Figure 7.5 plots the hot-spot mass-fraction contours for all the cases at $2.75 \mu \mathrm{s}$ for $v_{p}=250 \mathrm{~m} / \mathrm{s}$. Also shown are the corresponding profiles of the plastic heating rate $\overline{\dot{w}}_{p}$ whose magnitudes are normalized for convenience. For all the cases, most mass is locally heated through a fixed temperature rise $\Delta T_{b}=100 \mathrm{~K}$. The local mass-fraction that experiences a temperature rise between $5 \mathrm{~K}$ and $\Delta T_{b}$ varies between $10^{-1}$ and $10^{-3}$, respectively. For all friction coefficients, a second heating band can be identified on the contours which contains mass-fractions ranging between $10^{-3}$ to $10^{-4}$, with temperature rise between $\Delta T_{b}$ and $\Delta T_{f}=200 \mathrm{~K}$. Also, for $\mu>0.0$, small mass-fractions 


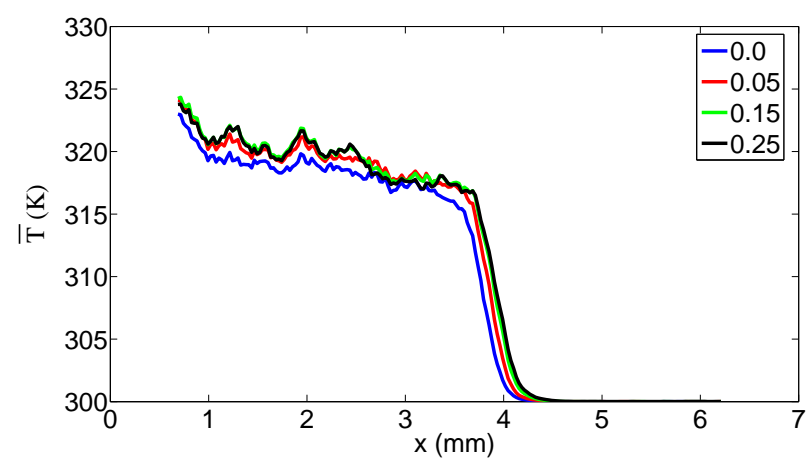

(a)

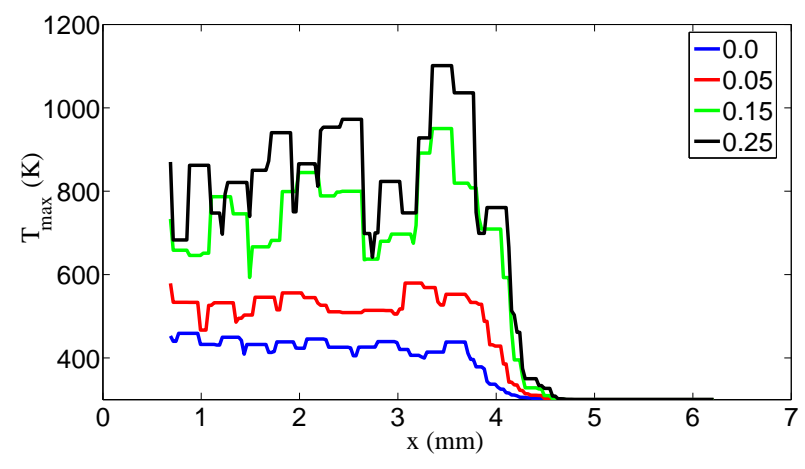

(b)

Figure 7.4: Predictions for the (a) average temperature $\bar{T}$ and (b) maximum temperature $T_{\max }$ for $v_{p}=250 \mathrm{~m} / \mathrm{s}$ at $2.75 \mu \mathrm{s}$ for $\mu=0.0,0.05,0.15$ and 0.25 .

$\left(m=10^{-5}\right)$ experience $\Delta T \geq \Delta T_{f}$, up to a maximum temperature rise of 300,650 , and $800 \mathrm{~K}$ for $\mu=0.05,0.15$, and 0.25 , respectively. These hot-spots vary sporadically with axial position and increase in number with friction coefficient. These predictions clearly indicate that the hot-spot number density is influenced by the friction coefficient, with a larger friction coefficient resulting in more hot-spots with larger temperature rises.

To further illustrate the influence of $\mu$ on hot-spot distribution, hot-spot mass-fractions are analyzed at fixed axial locations behind the compaction zone. Towards this end, the local massfraction for $v_{p}=50,250$ and $500 \mathrm{~m} / \mathrm{s}$ at a fixed axial position within the bed is plotted in Fig. 7.6. For each piston speed, this axial position corresponds to axial location (c) in Fig. 6.33. From the figures, it is evident that the hot-spot distributions for all the friction coefficients overlap for temperature rises below $\Delta T_{b} \approx 30,100$, and $200 \mathrm{~K}$ for $v_{p}=50,250$, and $500 \mathrm{~m} / \mathrm{s}$, respectively, with the largest disparities occurring in the tail end of the distributions. This is expected as the bulk of the distributions is representative of inelastic heating, which is not sensitive to $\mu$, whereas, the tail of the distributions is representative of frictional heating, which is sensitive to $\mu$. These predictions collectively highlight the influence of the friction coefficient on the hot-spot mass-fraction distributions, and particularly on the high-temperature end of the distributions, which are likely responsible for combustion initiation. 

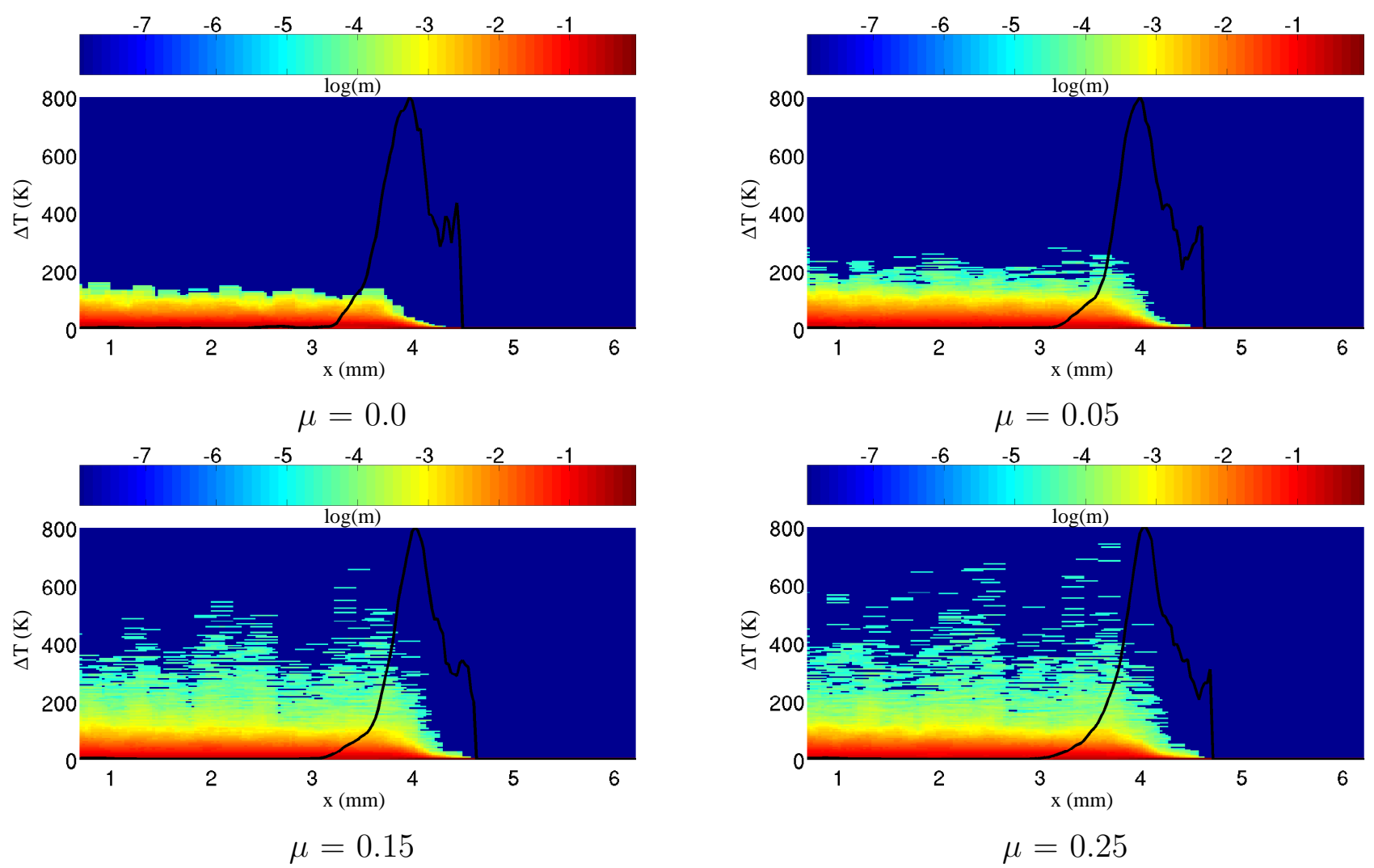

Figure 7.5: Predicted hot-spot mass-fraction contours for $v_{p}=250 \mathrm{~m} / \mathrm{s}$ at $2.75 \mu \mathrm{s}$ for $\mu=0.0$, $0.05,0.15$ and 0.25 . Values in the color-bar represent the logarithm of mass-fraction.

\section{$7.2 \quad$ Plastic Viscosity}

Predictions illustrating the influence of the viscosity parameter $\nu$ on the thermomechanical fields within the particle ensemble are presented in this section. In rate dependent viscoplastic constitutive theory, the material stress state is not confined to stay on or within the yield surface, rather, it is allowed to overshoot the yield surface and then relax back to it. In this formulation, $\nu$ determines the rate of relaxation of the stress state back to the yield surface. Rate independent behavior is approached for $\nu \rightarrow 0$, whereas, elastic behavior is recovered for $\nu \rightarrow \infty$. To demonstrate the influence of $\nu$ on the system thermomechanics a lower value for the viscosity parameter $\nu=10$ Pa.s is chosen, and a set of 10 simulations are performed by varying the piston speed between $v_{p}=50 \mathrm{~m} / \mathrm{s}$ to $500 \mathrm{~m} / \mathrm{s}$, in $50 \mathrm{~m} / \mathrm{s}$ increments. For each simulation, all other material and numerical parameters are held fixed at the baseline values listed in Table 4.1. Wave profiles and contour plots given in this section correspond to $t=2.75 \mu \mathrm{s}$ for the case $v_{p}=250 \mathrm{~m} / \mathrm{s}$, unless 


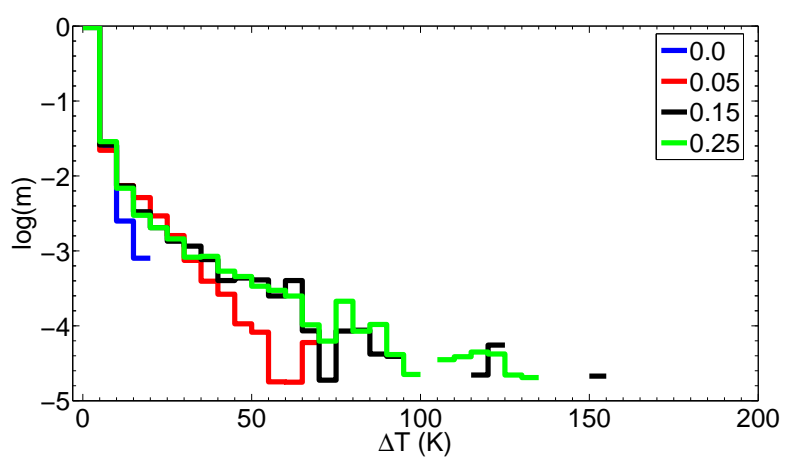

(a) $50 \mathrm{~m} / \mathrm{s}$

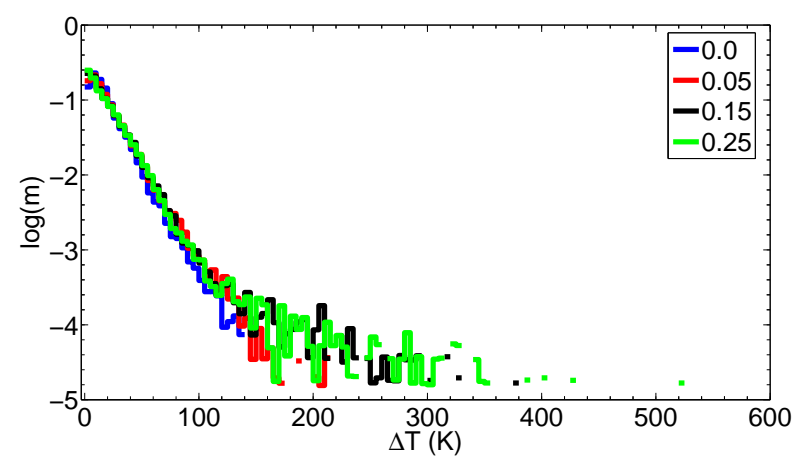

(b) $250 \mathrm{~m} / \mathrm{s}$

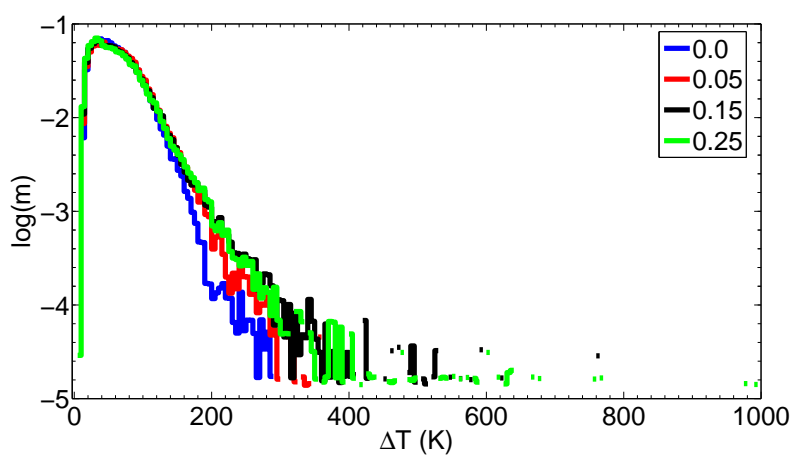

(c) $500 \mathrm{~m} / \mathrm{s}$

Figure 7.6: Predicted variation of hot-spot mass-fraction behind the compaction wave with $\mu$ for $v_{p}=50,250$ and $500 \mathrm{~m} / \mathrm{s}$ at $2.75 \mu \mathrm{s}$.

otherwise stated. For ease of presentation, in the remainder of this section the lower viscosity case and the baseline case will be referred to as case V-1 and V-2, respectively. The mechanical response is discussed first followed by the thermal response.

\subsubsection{Mechanical Response}

Figure 7.7(a) plots profiles of solid volume fraction for case $\mathrm{V}-1$ and $\mathrm{V}-2$. Lowering the viscosity parameter restricts the stress state from overshooting the yield surface by a large margin. Consequently, the inelastic deformation process occurs close to the yield surface. This results in larger plastic deformation rates following initial impact and therefore greater inelastic strains as the particles flow plastically. Therefore, the particle ensemble offers lesser resistance to compaction for case V-1 resulting in a larger quasi-steady final compacted value $\bar{\phi}_{s, f}$. This result is also reflected in Fig. 7.7(b) which plots the RMS fluctuation in the axial velocity for both the cases. Because the particles experience larger deformation rates within the compaction wave for case $\mathrm{V}-1$, the peak in 


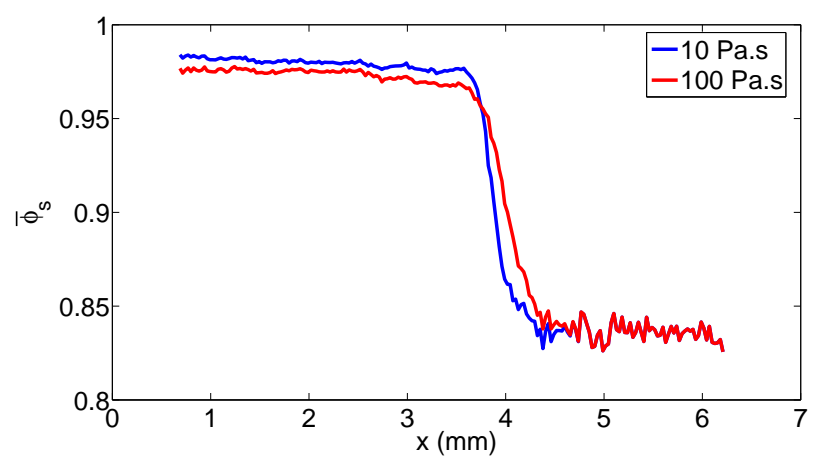

(a)

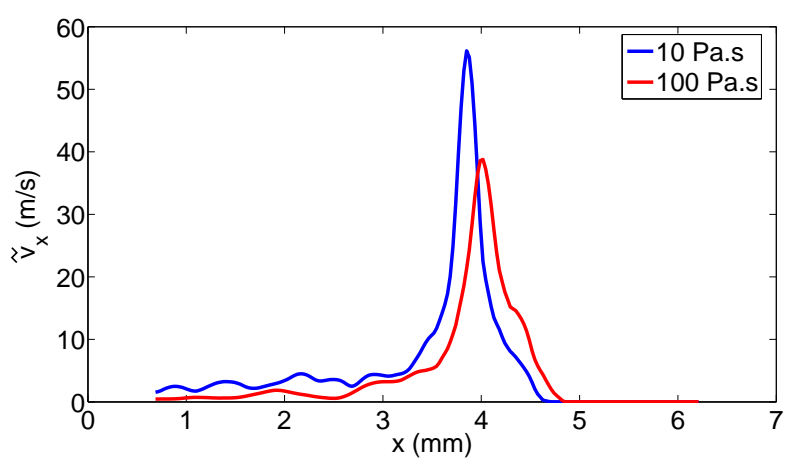

(b)

Figure 7.7: Predictions for the (a) average solid volume fraction $\bar{\phi}_{s}$ and (b) RMS fluctuation in axial velocity $\widetilde{v}_{x}$ at $2.75 \mu \mathrm{s}$ for $v_{p}=250 \mathrm{~m} / \mathrm{s}$ for $\nu=10$ and 100 Pa.s.

the axial velocity fluctuation profiles is also greater for the same case. Although not shown here, the average pressure and axial velocity profiles were found to be qualitatively and quantitatively similar for both cases. In this study, pressure is a function of volume change and temperature. Because the Von Mises yield criterion is pressure and temperature independent, pressure is unaffected by inelastic deformation, while $\nu$ only influences the inelastic material behavior. Subsequently, the pressure field is unaffected by variations in $\nu$.

To further illustrate the effect of $\nu$ on the plastic response of the particle ensemble, profiles of the average Von Mises stress $\overline{\boldsymbol{\tau}}_{e}$ and the average effective plastic strain $\bar{\epsilon}_{p}$ are plotted in Fig. 7.8 for case V-1 and V-2. Profiles of $\overline{\boldsymbol{\tau}}_{e}$ show that both the peak of the Von-Mises stress as well as its value behind the compaction wave are lower for case $\mathrm{V}-1$. This is expected because a lower viscosity value forces the inelastic deformation process to occur close to the yield surface which implies that the average equivalent stresses are also lower for case V-1. The influence of viscosity can be better analyzed by inspecting the time constant for the equivalent stress $\overline{\boldsymbol{\tau}}_{e}$ to relax back to the yield surface given by $\nu / G$. For a given piston speed, lower values of viscosity result in smaller time constants and lower overshoot. The wave profiles are thinner with sharper wave fronts. High values of viscosity result in dispersed wave structures. A lower value of viscosity also results in larger inelastic deformation rates, and subsequently larger inelastic strains, as evident in Fig. 7.8(b).

Figure 7.9 plots the variation in leading and trailing wave speeds with piston speed for case 


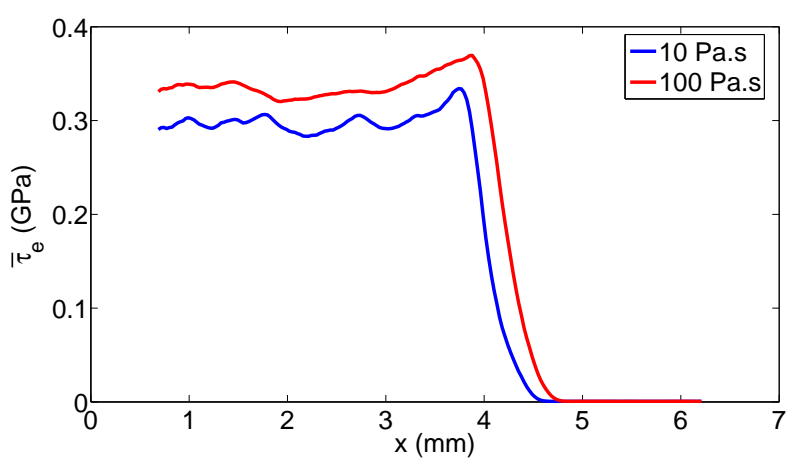

(a)

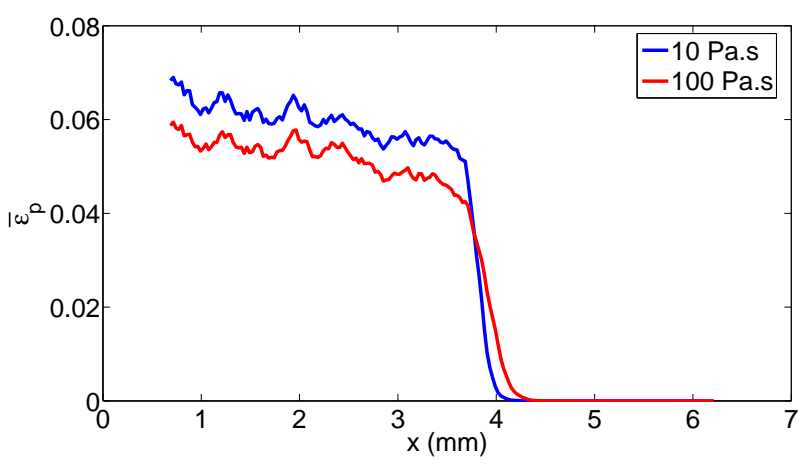

(b)

Figure 7.8: Predictions for the (a) average Von Mises stress $\overline{\boldsymbol{\tau}}_{e}$ and (b) average effective plastic strain $\bar{\epsilon}_{p}$ at $2.75 \mu \mathrm{s}$ for $v_{p}=250 \mathrm{~m} / \mathrm{s}$ for $\nu=10$ and 100 Pa.s.

V-1 and V-2. The predictions indicate that the leading and trailing wave speeds are marginally greater for the baseline cases with the largest disparity between the two cases occurring between $100 \mathrm{~m} / \mathrm{s} \leq v_{p} \leq 300 \mathrm{~m} / \mathrm{s}$. Due to the dynamic nature of the compaction process it is expected that the rate dependent relaxation behavior influences path dependent processes such as plastic work and friction work within the particle ensemble, which can further alter the compaction dynamics and influence compaction wave behavior. For example, increasing the viscosity increases the average normal contact pressure at the contact interfaces as the material response approaches elastic behavior. Subsequently, a larger tangential force is required to overcome stick. Therefore, the particle ensemble becomes frictionally rigid which results in faster wave speeds. Whereas, lower viscosity values result in lower inter-particle normal contact pressure and more slip. Subsequently, the particle ensemble offers lesser resistance to compaction. Because inelastic deformation is insignificant at very low piston speeds, $v_{p} \leq 100 \mathrm{~m} / \mathrm{s}$ case, these cases are not very sensitive to the viscosity parameter. For $v_{p} \geq 300 \mathrm{~m} / \mathrm{s}$, because all the porosity is squeezed out, the compaction dynamics and the wave speeds are relatively insensitive to viscosity.

\subsubsection{Thermal Response}

To demonstrate the sensitivity of the thermal response of the particle ensemble to $\nu$, the temperature field is first considered. Figure 7.10 plots the average and maximum temperature profiles for case V-1 and V-2. The predictions show that the average temperature profiles for both 


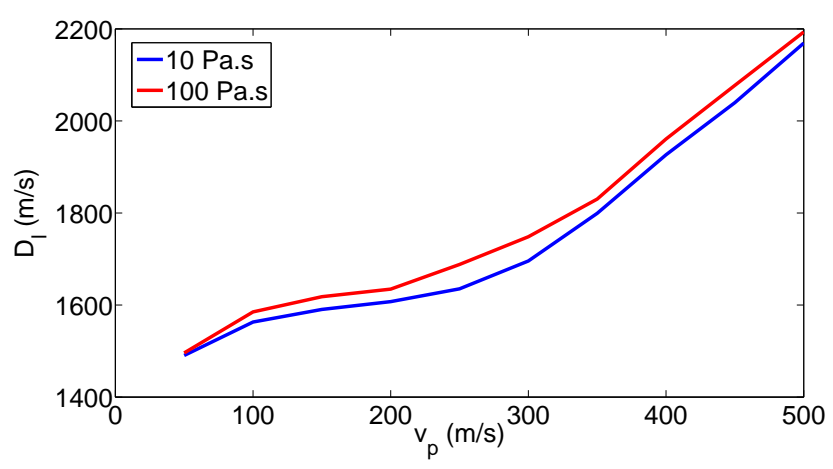

(a)

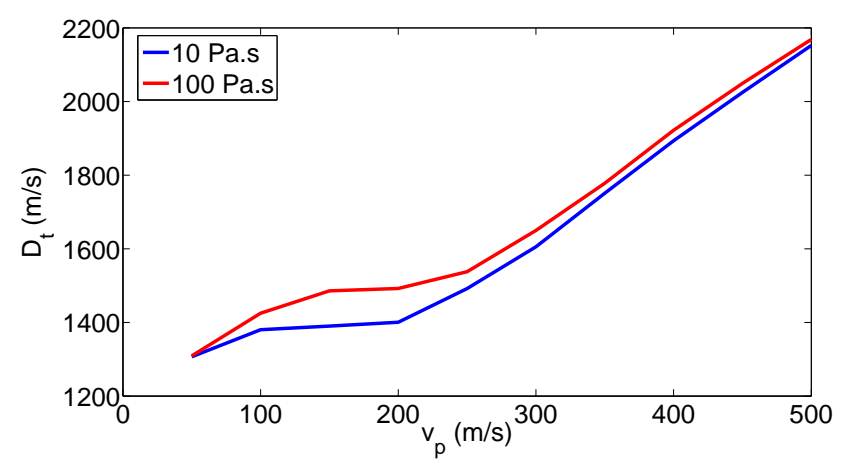

(b)

Figure 7.9: Predicted variation in (a) precursor $\left(D_{l}\right)$ and (b) plastic $\left(D_{t}\right)$ wave speed with piston speed $\left(v_{p}\right)$ for $\nu=10$ and 100 .

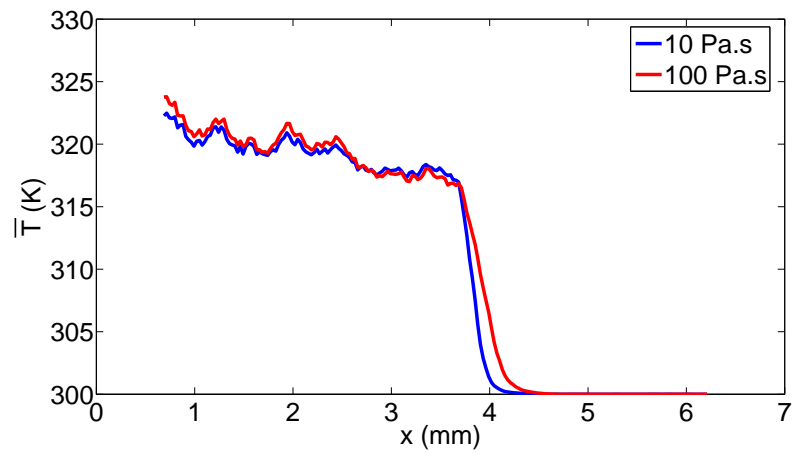

(a)

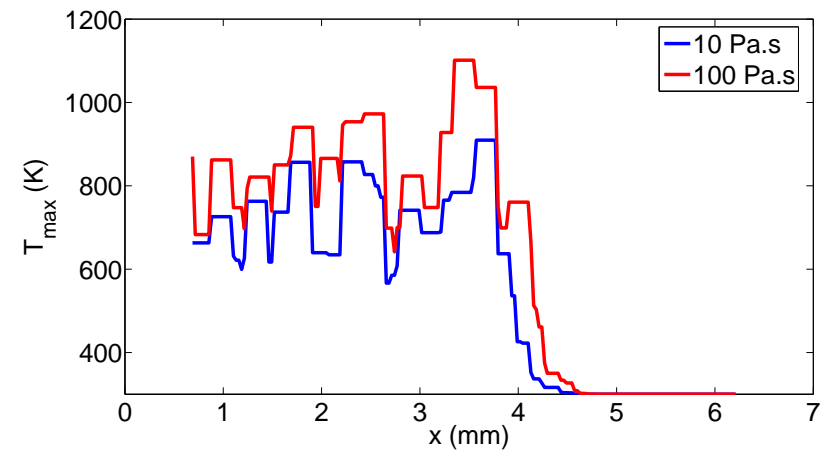

(b)

Figure 7.10: Predictions for the (a) average temperature $\bar{T}$ and (b) maximum temperature $T_{\max }$ for $v_{p}=250 \mathrm{~m} / \mathrm{s}$ at $2.75 \mu \mathrm{s}$ for $\nu=10$ and $100 \mathrm{~Pa} . \mathrm{s}$.

the cases are qualitatively and quantitatively similar indicating that the average temperatures, which are mostly representative of inelastic heating, are insensitive to the viscosity parameter. Although not shown here, the predictions for the mass specific plastic work were also found to be qualitatively and quantitatively similar for both cases. This is expected because the plastic work is proportional to the product of the effective stress $\bar{\tau}_{e}$ and the effective plastic strain $\bar{\epsilon}_{p}$, and although $\bar{\epsilon}_{p}$ is larger for case $\mathrm{V}-1 \bar{\tau}_{e}$ is smaller for the same case. Consequently, their product is similar for both cases. However, the maximum temperatures induced within the bed for the baseline case are substantially higher than the lower viscosity case indicating that the viscosity parameter has a substantial influence on frictional heating. Clearly, for $v_{p}=250 \mathrm{~m} / \mathrm{s}$, the maximum induced temperatures decrease from $1100 \mathrm{~K}$ for case $\mathrm{V}-2$ to $900 \mathrm{k}$ for case $\mathrm{V}-1$. 

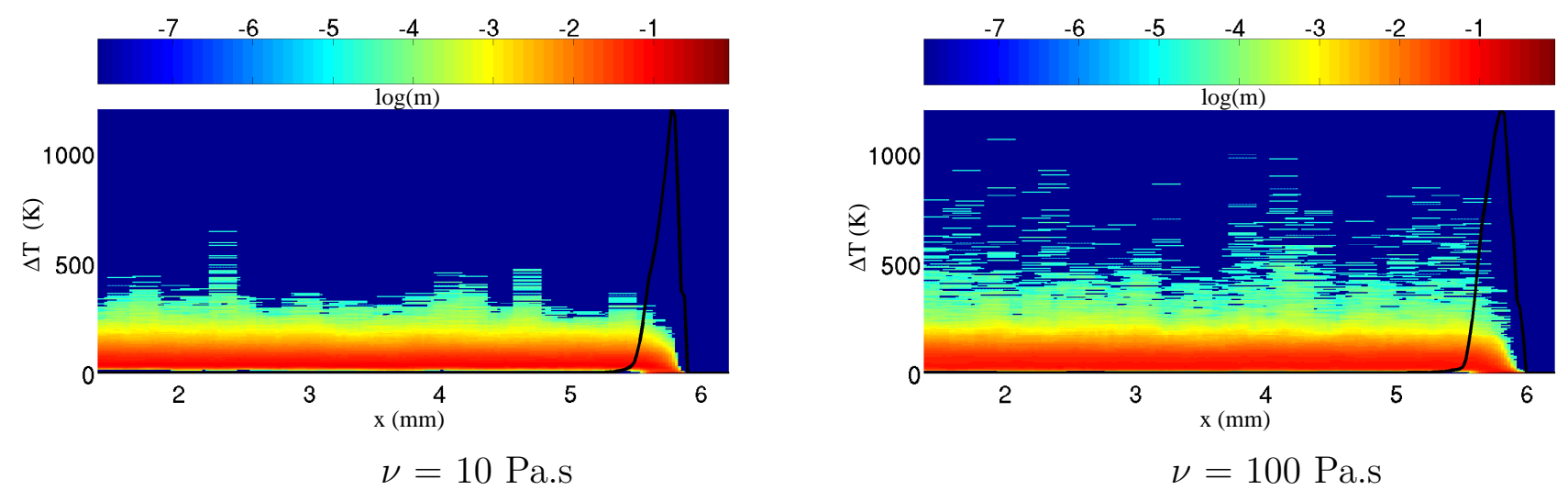

Figure 7.11: Predicted hot-spot mass-fraction contours for $v_{p}=500 \mathrm{~m} / \mathrm{s}$ at $2.75 \mu \mathrm{s}$ for $\nu=10$ and 100 Pa.s. Values in the color-bar represent the logarithm of mass-fraction.

The influence of the viscosity parameter on the hot-spot mass-fraction distribution is now considered. Decreasing the viscosity parameter results in lower average normal contact forces at inter-particle contact interfaces as the material response approaches rate independent behavior. Lower normal forces result in significantly lower frictional dissipation for the lower viscosity cases. Subsequently, the maximum temperatures obtained in these cases are lower than their baseline counterparts. Plotted in Fig. 7.11 are the hot-spot mass-fraction contours for $v_{p}=500 \mathrm{~m} / \mathrm{s}$ for case V-1 and V-2 at $2.75 \mu \mathrm{s}$. For both cases, local mass-fractions of the order $10^{-1}-10^{-3}$ experience a temperature rise between $5 \mathrm{~K}$ and $\Delta T_{b}=200 \mathrm{~K}$. Substantial differences are observed in the number of hot-spots with mass-fractions of the order $10^{-3}$ to $10^{-4}$ that are heated to a temperature rise between $\Delta T_{b}$ and $\Delta T_{f}=400 \mathrm{~K}$, and in mass-fractions of order $10^{-5}$ that experience $\Delta T \geq \Delta T_{f}$. These hot-spots, which are primarily formed due to friction work, decrease in number for case $\mathrm{V}-1$.

To further illustrate the influence of the viscosity parameter on hot-spot distribution, local hot-spot mass- fractions at a fixed axial position within the bed are plotted in Fig. 7.12 for $v_{p}=$ 50, 250 and $500 \mathrm{~m} / \mathrm{s}$. For each piston speed, the axial position within the bed corresponds to axial location (c) in Fig. 6.33. For all the piston speeds, the hot-spot distribution for both viscosities display similar qualitative and quantitative features for $\Delta T \leq \Delta T_{b}$. This result indicates that $\nu$ has minimal influence on hot-spots that are primarily formed due to plastic work. However, significant differences are observed in hot-spots heated to $\Delta T>\Delta T_{b}$ between cases $\mathrm{V}-1$ and $\mathrm{V}-2$, 


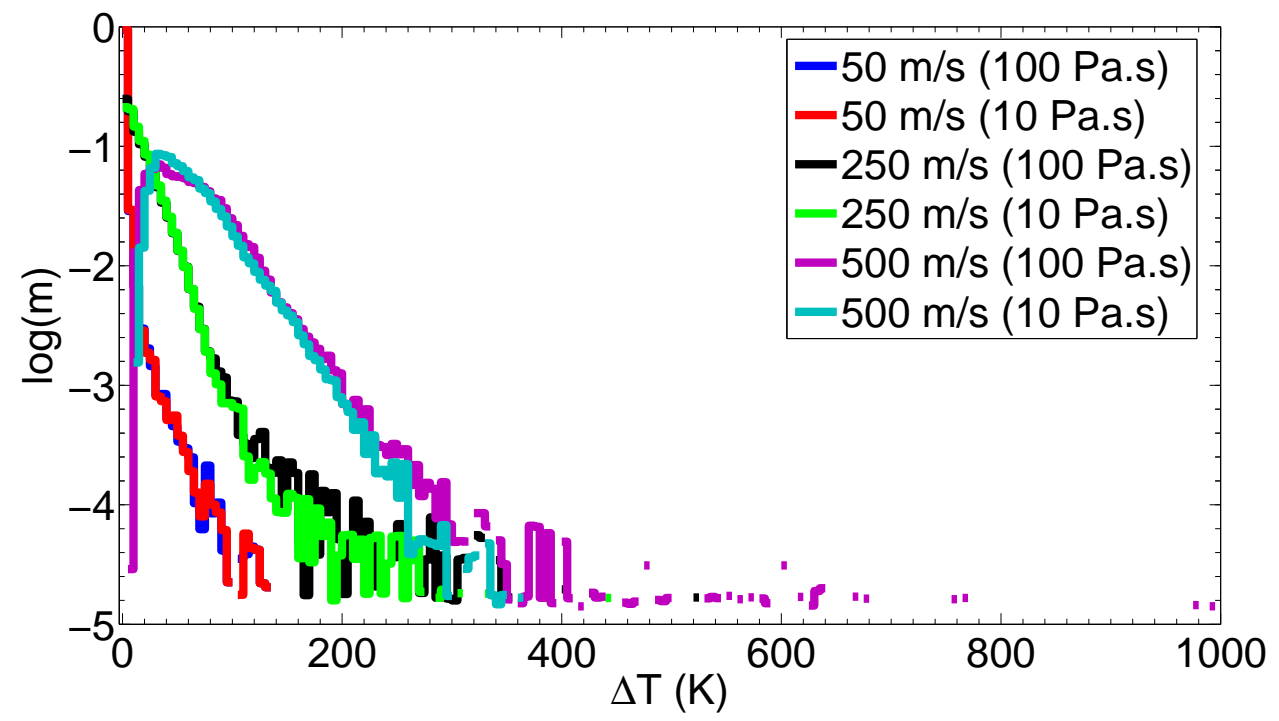

Figure 7.12: Predicted variation of hot-spot mass-fraction behind the compaction wave with $\nu$ for $v_{p}=50,250$ and $500 \mathrm{~m} / \mathrm{s}$ at $2.75 \mu \mathrm{s}$.

and these differences becomes more prominent with increasing piston speed. For example, for $v_{p}=50 \mathrm{~m} / \mathrm{s}$, mass-fractions of the order $10^{-5}$ are heated through a maximum temperature rise of $130 \mathrm{~K}$ for both viscosities. Whereas, for $v_{p}=500 \mathrm{~m} / \mathrm{s}$, mass-fractions of the same order are heated through a maximum temperature rise of $1100 \mathrm{~K}$ for case V-2 and only $440 \mathrm{~K}$ for case V-1. These predictions collectively highlight the influence of the viscosity parameter on the hot-spot distribution, and particularly on the high temperature end of the distributions that are induced due to friction work.

\subsection{Particle Size Distribution}

Sensitivity of the thermomechanical response of the particle ensemble to particle size distribution is demonstrated in this section. To this end, ten simulations are performed on an alternate granular ensemble, as shown in Fig. 7.13, by varying the piston speed from $50 \mathrm{~m} / \mathrm{s}$ to $500 \mathrm{~m} / \mathrm{s}$, in $50 \mathrm{~m} / \mathrm{s}$ increments. In this section, for tractability, the baseline configuration will be referred to as S-1 and the alternate configuration as S-2. Configuration S-2 also consists of 2000, tightly packed, randomly distributed circular particles inside a rectangular domain, which is approximately $1.5 \mathrm{~mm}$ in width and $10.34 \mathrm{~mm}$ in length. Shown in Fig. 7.14 is the particle size distribution for both 


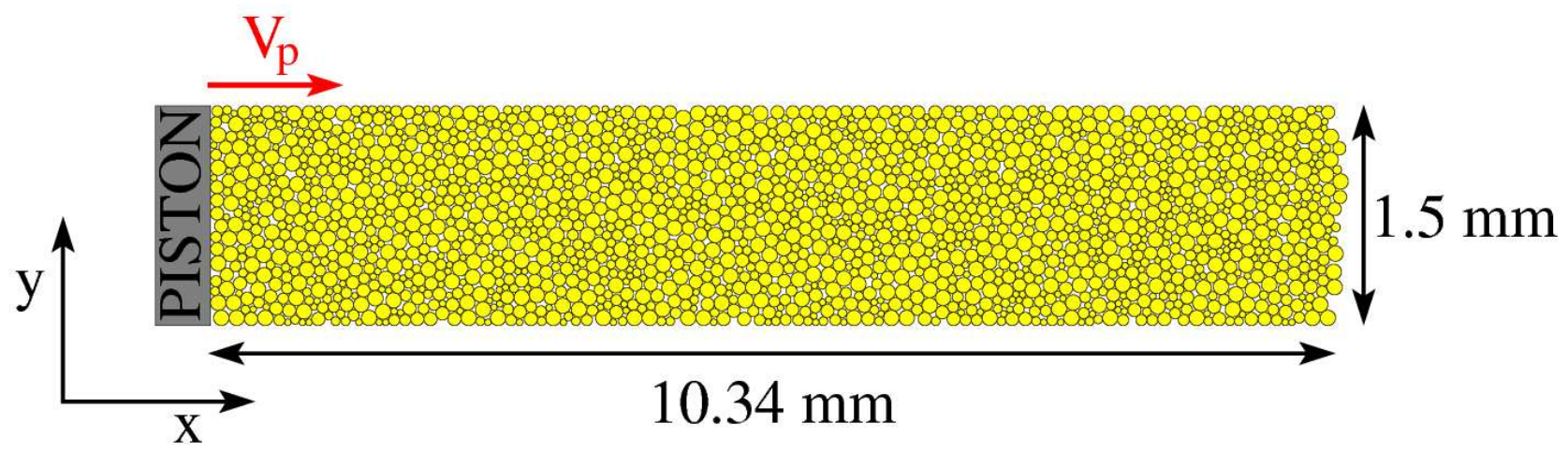

Figure 7.13: Schematic of the alternate initial system configuration used in this study.

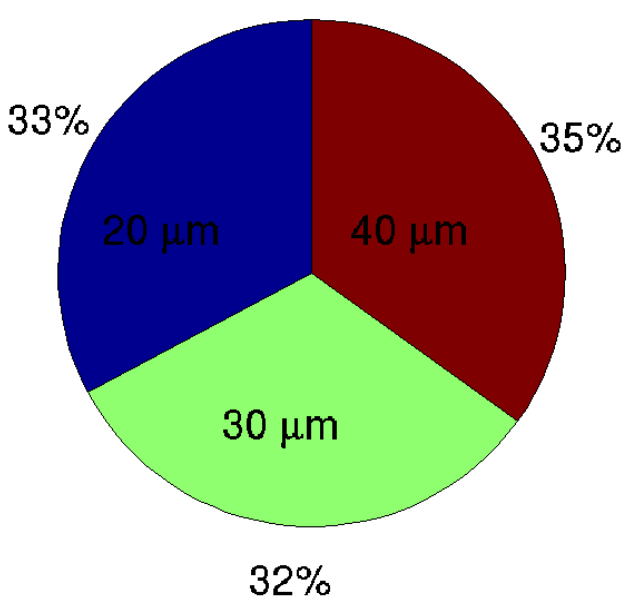

(a)

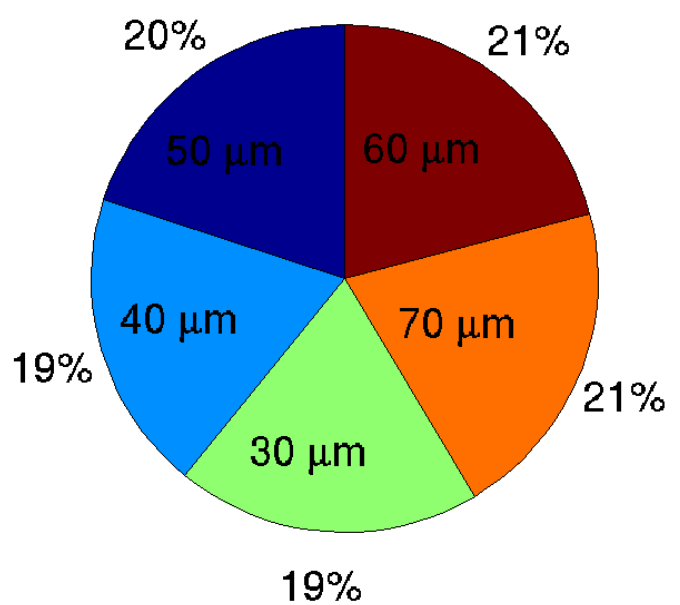

(b)

Figure 7.14: Initial particle size distribution for (a) Configuration S-1 and (b) Configuration S-2. configurations. For configuration S-2, the initial particle radius was chosen from values of 30, 40, 50, 60 and $70 \mu \mathrm{m}$. This distribution corresponds to a conventional-coarse nonuniform distribution with mean radius of $50 \mu \mathrm{m}$. Each particle was discretized using CST finite elements, where all the finite elements had a uniform element edge length of $3 \mu \mathrm{m}$. The particle ensemble consisted of 2330923 elements and 1267385 nodes. Depending on its initial radius, each particle consisted of 453 to 1870 finite elements.

Figure 7.15 shows the initial solid volume fraction profile, $\bar{\phi}_{s, 0}(x)$, for both configurations. Configuration S-2 has an average solid volume fraction of $83.8 \%$, with global minimum and maximum values of $81.5 \%$ and $86.0 \%$, respectively. Both configurations have similar initial solid volume 


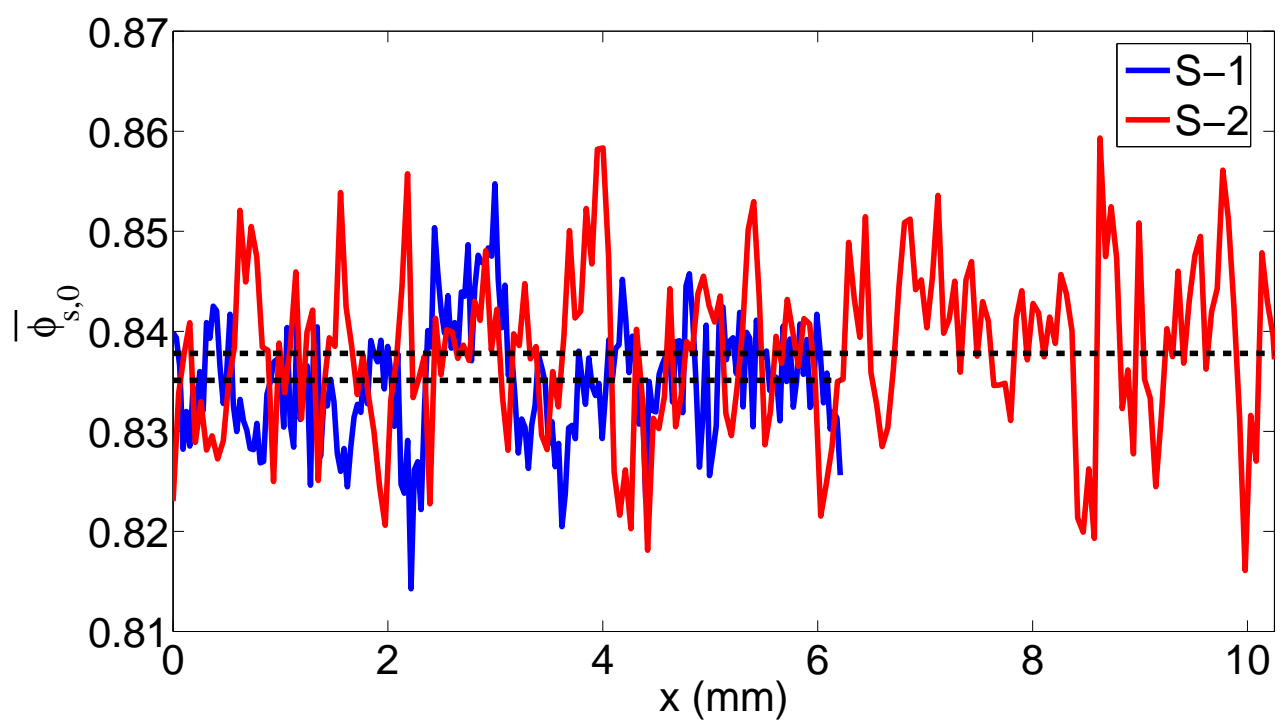

Figure 7.15: Average initial solid volume fraction $\bar{\phi}_{s, 0}$ for configuration S-1 and S-2.

fraction, and the initial porosity profiles for both configurations display short wavelength, large amplitude fluctuations due to the heterogeneities. However, the amplitude of the fluctuations is larger for configuration S-2 indicating that configuration S-2 exhibits additional local heterogeneities due to a wider particle size distribution.

\subsubsection{Mechanical Response}

Predictions for the mechanical response of configuration S-1 and S-2 displayed similar qualitative and quantitative trends with a few marginal differences. Shown in Fig. 7.16(a) are the predicted solid volume fraction profiles for $v_{p}=250 \mathrm{~m} / \mathrm{s}$ at $3.5 \mu \mathrm{s}$ for both configurations. From the figure, it is evident that while the solid volume fraction profiles for both configurations are qualitatively similar the final quasi-steady solid volume fraction behind the compaction wave is marginally greater for configuration S-2. As indicated by the initial porosity profiles, the mesostructure of configuration S-2 exhibits more local heterogeneity than S-1. Subsequently, in this configuration, particles have more mobility as the smaller particles are able to deform and occupy voids between larger particles, and therefore this particle ensemble offers lesser resistance to compaction. This result is further evident in Fig. 7.17, which plots the average pressure profiles for each particle size class for both configurations. For each particle size class, the corresponding 


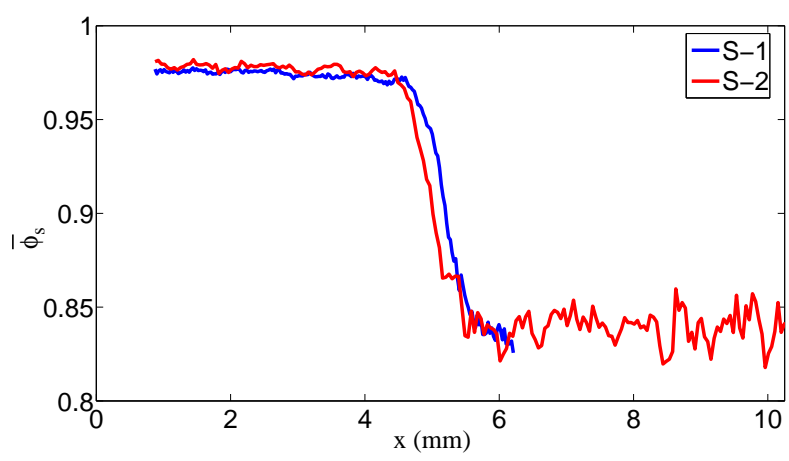

(a)

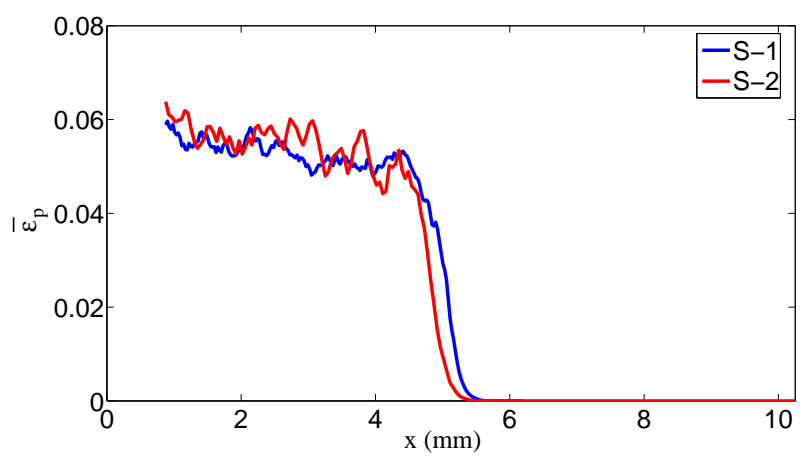

(b)

Figure 7.16: Predictions for the (a) average solid volume fraction $\bar{\phi}_{s}$ and (b) average effective plastic strain $\bar{\epsilon}_{p}$ at $2.75 \mu \mathrm{s}$ for $v_{p}=250 \mathrm{~m} / \mathrm{s}$ for configuration S-1 and S-2.

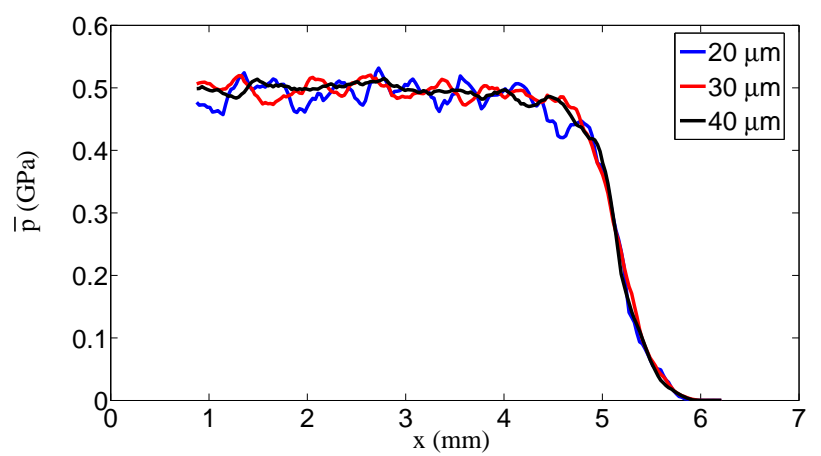

S-1

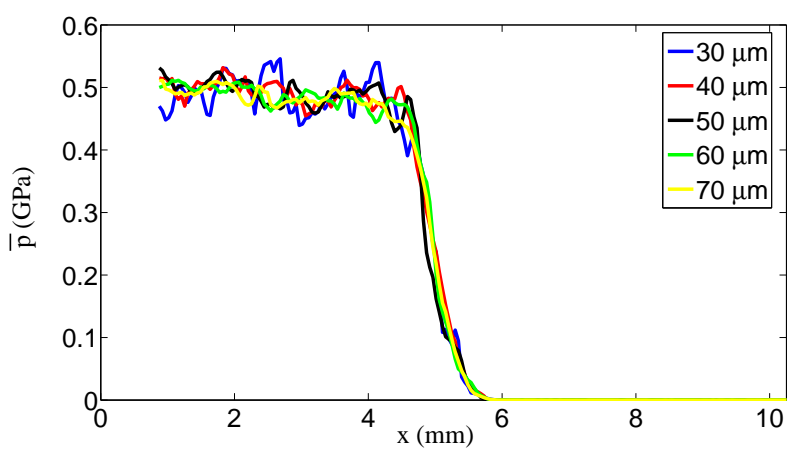

S-2

Figure 7.17: Predictions for the average pressure $\bar{p}$ for all particle size classes at $2.75 \mu \mathrm{s}$ for $v_{p}=$ $250 \mathrm{~m} / \mathrm{s}$ for configuration S-1 and S-2.

average profiles were obtained by averaging over the particles corresponding to that particular initial particle size. Subsequently, three different profiles are obtained for configuration S-1 and five for configuration S-2. From the figure, it is evident that the average quasi-steady pressure behind the compaction wave is similar for all particle sizes. However, heterogeneities in the mesostructure introduce oscillations in the pressure profiles for both configurations. The amplitude of these oscillations increases with decreasing initial particle size and is more readily apparent in configuration S-2 than S-1. This result indicates that while the average pressure response is insensitive to particle size distribution, spatial fluctuations in the stress state are more prominent in the smaller particles, which may be shielded from the wave by larger particles.

To compare the plastic response of the two configurations, profiles of effective plastic strain are 


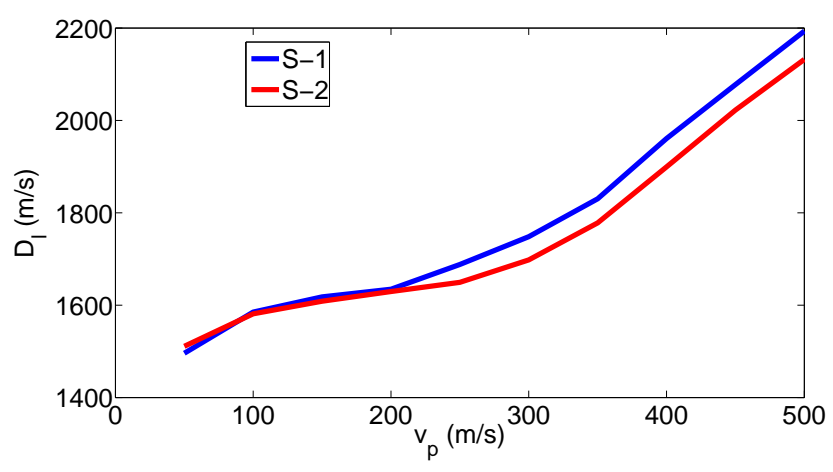

(a)

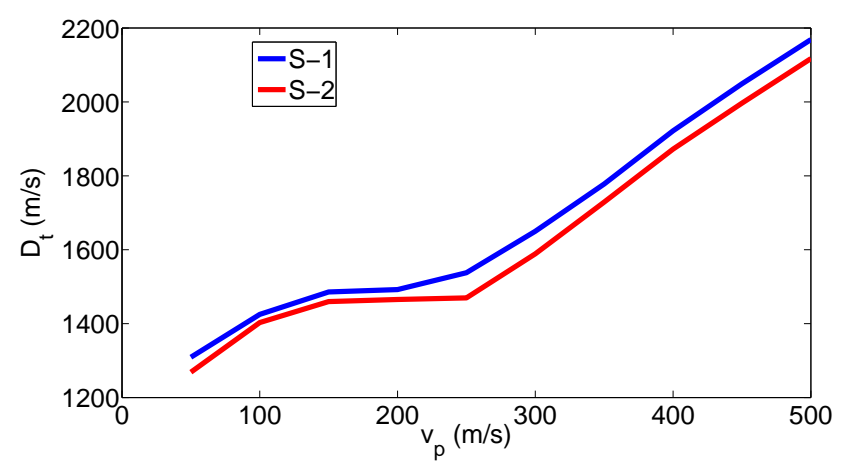

(b)

Figure 7.18: Predicted variation in (a) precursor $\left(D_{l}\right)$ and (b) plastic $\left(D_{t}\right)$ wave speed with piston speed $\left(v_{p}\right)$ for configuration S-1 and S-2.

plotted in Fig. 7.16(b) for the $250 \mathrm{~m} / \mathrm{s}$ case at $3.5 \mu \mathrm{s}$. From the figure, it is evident that although the plastic strain profile for configuration S-2 displays more fluctuations than S-1 due to increased heterogeneity, their average plastic response is similar indicating that the plastic response of the particle ensemble is insensitive to the chosen particle size distribution. Although not shown here, the profiles of equivalent Von Mises stress for both configurations also displayed similar qualitative and quantitative trends. Shown in Figure [7.18] is the variation in the leading and the trailing wave speeds with piston speed for both the configurations. Configuration S-2, which has a wider initial particle size distribution, offers lesser resistance to compaction. As a result, the compaction wave is marginally slower for configuration S-2. However, at low piston speeds $\left(v_{p} \leq 200 \mathrm{~m} / \mathrm{s}\right)$, the wave speeds are similar for both configurations.

\subsubsection{Thermal Response}

To describe the influence of particle size distribution on the thermal response of the particle ensemble, the temperature field is first considered. Shown in Fig. 7.20 are the average and maximum temperature profiles for $v_{p}=250 \mathrm{~m} / \mathrm{s}$ at $3.5 \mu$ s for both configurations. Similar to the effective plastic strain profiles shown in Fig. 7.16(b), the average temperature profiles for both configurations also display similar qualitative and quantitative trends, with the predictions for configuration S-2 showing larger fluctuations than S-1 due to additional heterogeneities. Because the average temperature profiles are largely representative of inelastic heating effects, these 


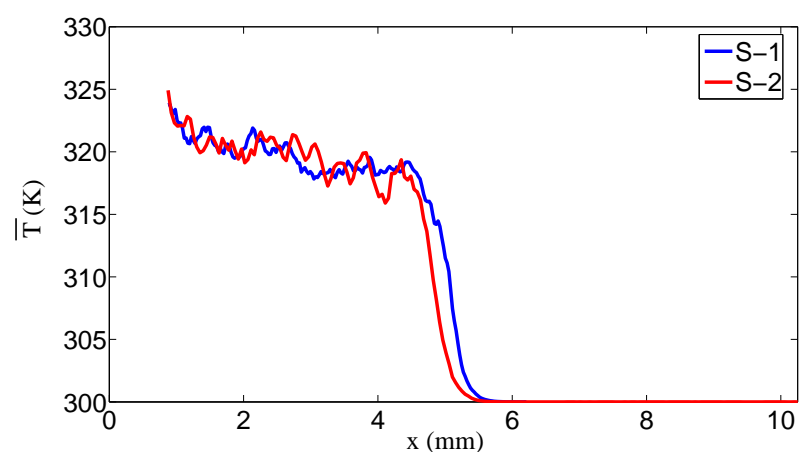

(a)

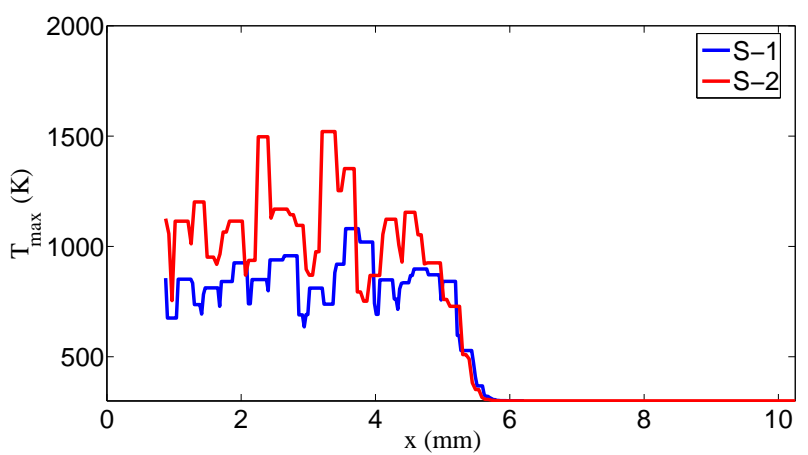

(b)

Figure 7.19: Predictions for the (a) average temperature $\bar{T}$ and (b) maximum temperature $T_{\max }$ for $v_{p}=250 \mathrm{~m} / \mathrm{s}$ at $2.75 \mu \mathrm{s}$ for configuration $\mathrm{S}-1$ and $\mathrm{S}-2$.

predictions further indicate that the plastic response of the particle ensemble is only marginally sensitive to the particle size distribution. However, substantial differences are observed in the maximum temperature profiles. A wider particle size distribution enhances frictional dissipation and results in larger frictional heating rates. This results in greater local temperatures $(\approx 1500 \mathrm{~K})$ for configuration S-2 as compared to that for configuration S-1 $(\approx 1100 \mathrm{~K})$. Furthermore, it is expected that additional heterogeneities introduced due to a wider size distribution, particle shape and the presence of multi-material constituents will further enhance frictionally induced hot-spot temperatures.

Shown in Fig. 7.20 are profiles of average temperature for each particle size class for both configurations. Unlike the predictions for the average pressure profiles shown in Fig. 7.17, the predictions for the average temperature profiles display high sensitivity to particle size class. The quasi-steady temperatures behind the compaction wave decrease with increase in initial particle size indicating that smaller particles posses more thermal energy per unit mass than larger particles. For both configurations, although regions of high temperatures induced due to friction and plastic work exist close to the boundaries of all the particles, cooler regions persist in the interior of larger particles which are unaffected by plastic work. Subsequently, the smaller particles possess greater thermal energy per unit mass.

To further illustrate the influence of particle size distribution on the thermal response, hotspot mass-fraction distributions are considered. Shown in Fig. 7.21 are the hot-spot mass-fraction 


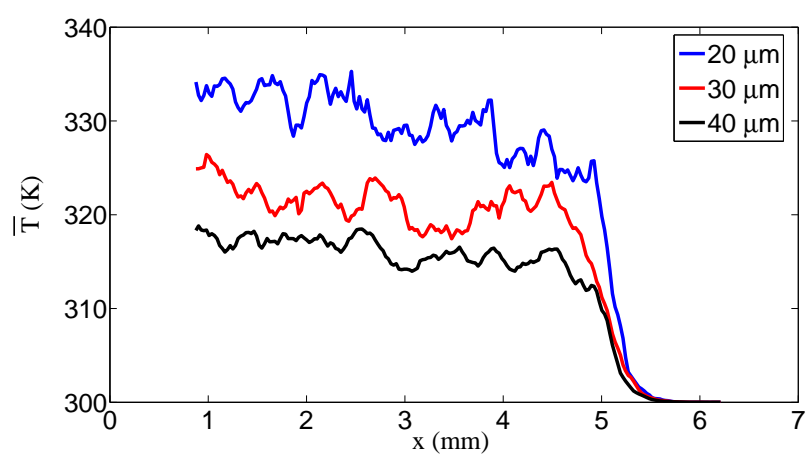

S-1

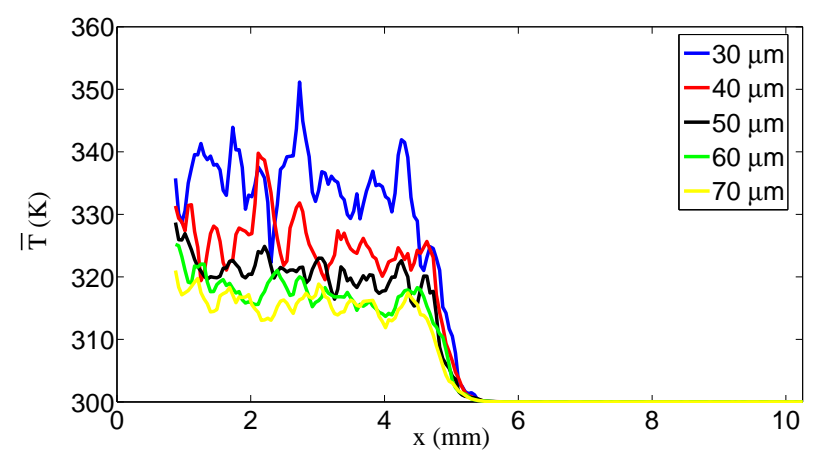

S-2

Figure 7.20: Predictions for the average temperature $\bar{T}$ for all particle size classes at $2.75 \mu \mathrm{s}$ for $v_{p}=250 \mathrm{~m} / \mathrm{s}$ for configuration $\mathrm{S}-1$ and $\mathrm{S}-2$.

contours for $v_{p}=50,250$ and $500 \mathrm{~m} / \mathrm{s}$ for both configurations. The contours are shown at $3.5 \mu \mathrm{s}$ for the 50 and $250 \mathrm{~m} / \mathrm{s}$ cases and at $2.75 \mu \mathrm{s}$ for the $500 \mathrm{~m} / \mathrm{s}$ case. For all piston speeds, local mass-fractions of the order $10^{-1}$ and $10^{-3}$ experience a temperature rise between $5 \mathrm{~K}$ and $\Delta T_{b}$, and local mass-fractions of the order $10^{-3}$ to $10^{-4}$ that are heated to a temperature rise between $\Delta T_{b}$ and $\Delta T_{f}$. From the figures, it is evident that particle size distribution has little influence on mass-fractions heated to temperature rises below $\Delta T_{f}$. However, substantial differences are observed in the number and temperatures of mass-fractions of order $10^{-5}$ that experience $\Delta T \geq$ $\Delta T_{f}$. For all piston speeds, larger temperature rises are induced within the compaction region for configuration S-2. Even for $v_{p}=50 \mathrm{~m} / \mathrm{s}$, local mass is heated to $900 \mathrm{~K}$ for configuration S-2, which increase to 1550 and $1700 \mathrm{~K}$ for the 250 and $500 \mathrm{~m} / \mathrm{s}$ cases, respectively. Figure [7.22] plots the local mass-fraction for $v_{p}=50,250$ and $500 \mathrm{~m} / \mathrm{s}$ behind the compaction wave corresponding to axial location (c) in Fig. 6.33. The figure shows that, for each piston speed, the bulk hot-spot mass-fraction distribution is qualitatively and quantitatively similar indicating that the plastic response is relatively insensitive to the chosen particle size distribution. Perhaps a wider distribution may be required to more accurately describe the inelastic heating response to particle size. However, substantial differences are observed in the high temperature end of the hot-spot distribution indicating that the frictional response is highly sensitive to the particle size distribution.

Figure 7.23 plots the local hot-spot mass-fraction distribution for each particle size at a fixed 
$50 \mathrm{~m} / \mathrm{s}$
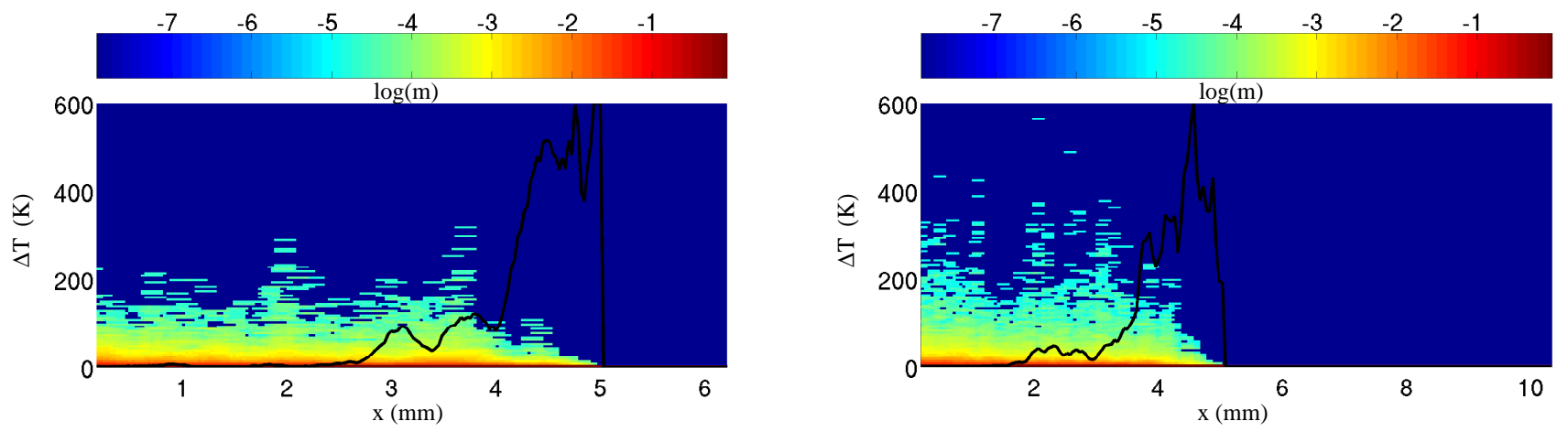

$250 \mathrm{~m} / \mathrm{s}$
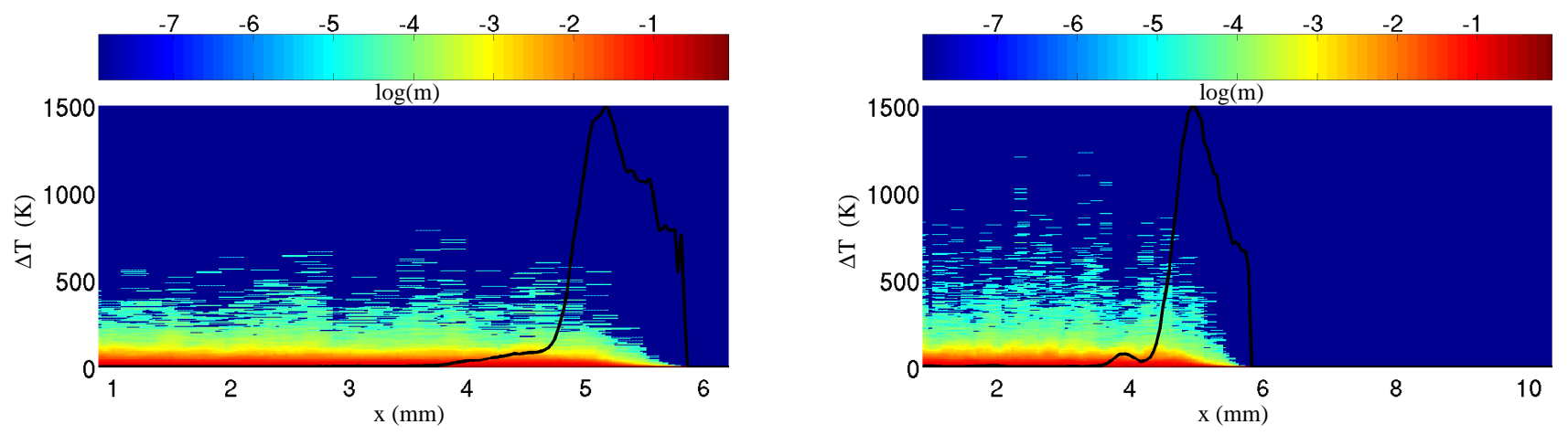

$500 \mathrm{~m} / \mathrm{s}$

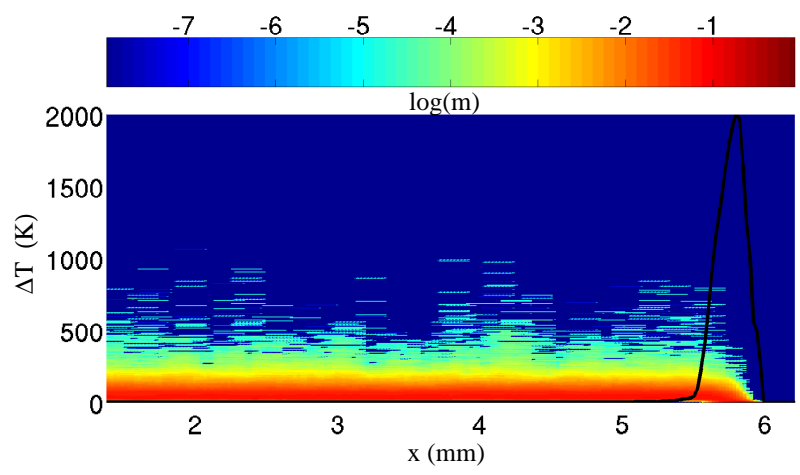

S-1

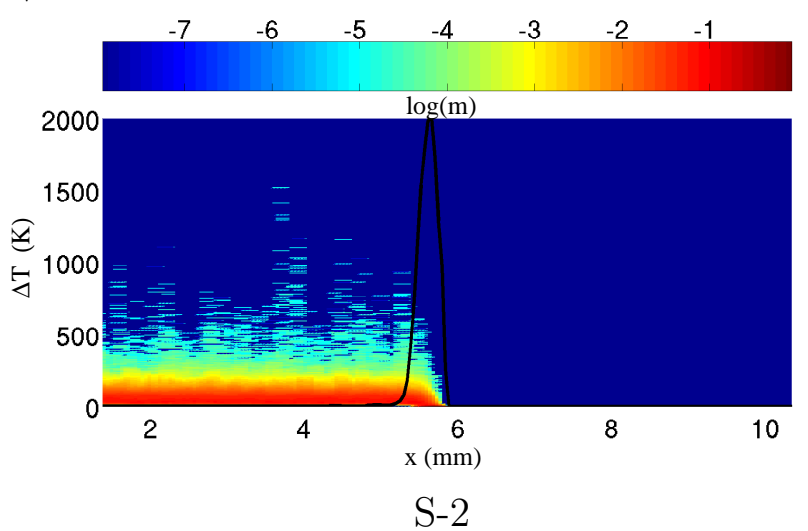

Figure 7.21: Predicted hot-spot mass-fraction contours for $v_{p}=50,250$ and $500 \mathrm{~m} / \mathrm{s}$ at $2.75 \mu \mathrm{s}$ for configuration S-1 and S-2. Values in the color-bar represent the logarithm of mass-fraction. 


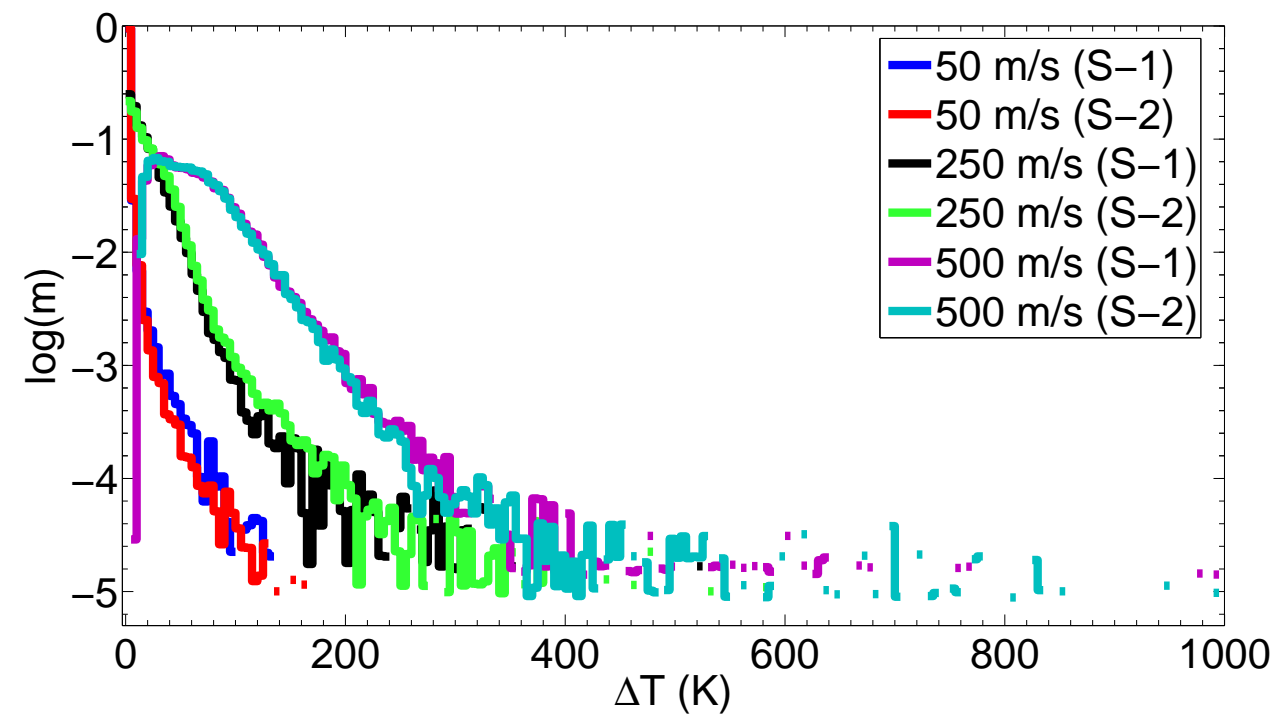

Figure 7.22: Predicted variation of hot-spot mass-fraction behind the compaction wave with particle size distribution for $v_{p}=50,250$ and $500 \mathrm{~m} / \mathrm{s}$ at $2.75 \mu \mathrm{s}$.

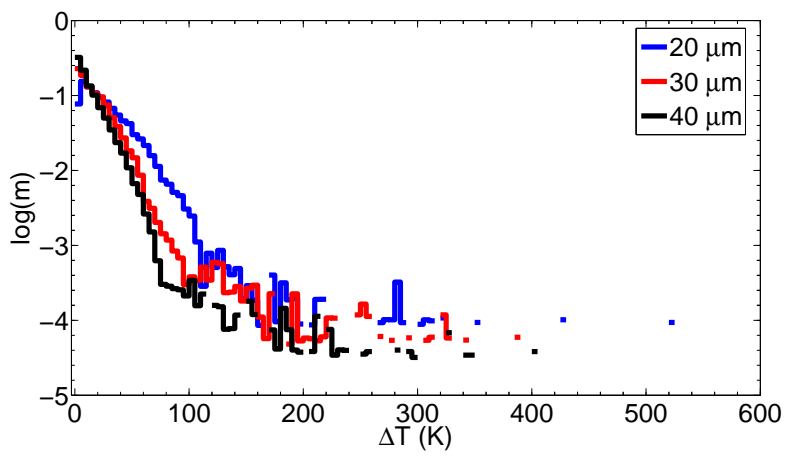

S-1

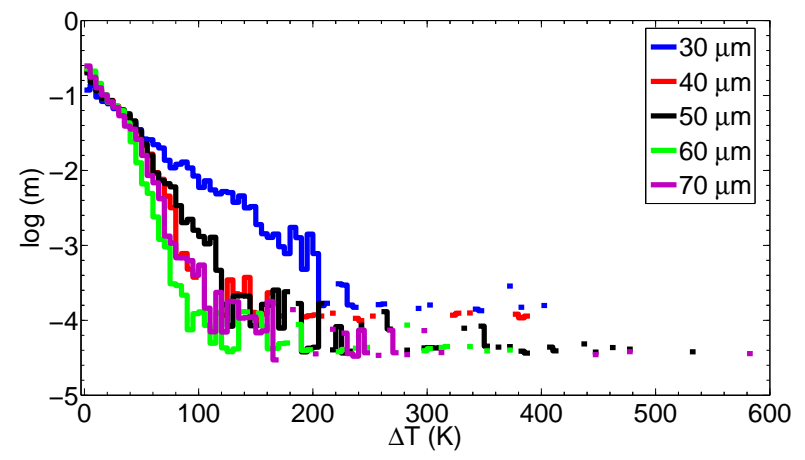

S-2

Figure 7.23: Predicted variation of hot-spot mass-fraction behind the compaction wave for each particle size class for $v_{p}=250 \mathrm{~m} / \mathrm{s}$ at $2.75 \mu \mathrm{s}$ for configuration S-1 and S-2. 
axial location corresponding to axial position (c) in Fig. 6.33. For each particle size class, the hot-spot mass-fraction distributions were obtained by considering only the mass of the particles corresponding to that particular initial particle size. The figure shows that while the smaller particles have a larger mass-fraction heated to temperatures below $\Delta T_{b}$, mass-fractions corresponding to the high temperature end of the distribution are associated with the larger particles. Therefore, the smaller particles have larger average temperatures, whereas, the larger particles experience the maximum local temperature rises. This result suggests that the larger particles may play a significant role in combustion initiation and the smaller particles may be important for reaction propagation. 


\section{Chapter 8}

\section{Conclusions and Future Work}

A 2D, Lagrangian, combined discrete and finite-element technique was formulated and computationally implemented to model impact induced thermomechanical interactions between deformable particles contained within the meso-structure of heterogeneous energetic solids. An energy consistent, penalty based, distributed potential force method was used to estimate the magnitude of normal forces at inter-particle contact surfaces, and a penalty regularized AmontonCoulomb law was used to model tangential forces due to friction. For ease of implementation, a new computational strategy was developed to enforce thermal contact constraints in a manner similar to that used for the kinematic constraints. A finite strain, thermoelastic-viscoplastic constitutive theory was used to describe particle deformation. The numerical technique was verified against known analytical solutions to simple problems, and predictions given by the well-established impact mechanics software Ls-Dyna for more complex problems. Ls-dyna uses a conventional penalty based master-slave interaction model to estimate contact forces which is useful for solving impactrelease problems. However, for sustained high pressure contact, the method leads to excessive penetration and numerical distortion of strain fields near the contact interface. To avoid these issues, a modified distributed potential force method is implemented in this study, which gives a more realistic distribution of contact forces for sustained contact. The computational model was first used to examine energy partitioning within a close-packed micro-particle cluster due to

impact with a rigid, planar wall, and then used to examine impact induced uniaxial, quasi-steady deformation waves within large, close-packed particle ensembles. Conclusions drawn from these studies and a brief discussion of future work are given in this chapter.

\subsection{Micro-Particle Cluster Impact}

Impact of an initially stress free, close-packed, 2D micro-particle cluster, consisting of 25 well-resolved particles of radii $50 \mu \mathrm{m}$, and a planar, rigid wall was computationally examined to 
characterize the influence of impact angle and packing configuration on the spatial and temporal partitioning of dissipated energy within the cluster. This study is relevant to the ignition of small, shock dispersed, heterogeneous, reactive micro-fragments by impact with wall boundaries. For a representative impact speed of $300 \mathrm{~m} / \mathrm{s}$, plastic work was shown to rapidly increase near the wall immediately following impact before diffusing outward due to stress transmission between colliding particles, whereas friction work near the wall increased sharply after a very brief initial period of particle-wall sticking ( $\approx 75$ ns for normal impact). The extent of sticking was largest for normal impact, and it decreased with increasing impact angle. The maximum average plastic work was approximately $4 \mathrm{~kJ}$ per unit cluster mass for an impact angle of $\phi^{\circ}=0^{\circ}$ (corresponding to normal impact), which was approximately two times larger than that for $\phi^{\circ}=60^{\circ}$. The maximum average friction work was approximately $60 \mathrm{~kJ}$ per unit cluster mass for $\phi_{c}^{\circ}=60^{\circ}$, which was nine times larger than that for $\phi^{\circ}=0^{\circ}$. The critical impact angle for maximum friction work was shown to mildly depend on the initially random cluster configuration, though it is anticipated that little variation in this angle would result for larger particle ensembles. Friction was shown to significantly affect the final cluster kinetic energy, but to minimally affect elastic potential energy and plastic work. Also, particle-wall friction was considerably larger than inter-particle friction due to both the rigid wall assumption and the repeated loading of particles adjacent to the wall by surrounding particles.

Predictions from an energy analysis indicated that large temperature rises (i.e., $\Delta T \geq 900 \mathrm{~K}$ ) occur within the cluster, even for normal impact, with the maximum local temperature $(\approx 4400 \mathrm{~K})$ occurring for $\phi^{\circ}=80^{\circ}$. However, the mass heated to such elevated temperatures represented less than $0.01 \%$ of the cluster mass, with most mass $(\approx 98 \%)$ experiencing temperatures rises of $\Delta T \leq 200 K$. Localization of dissipated energy indicated that average temperature rises due to friction work exceeded those due to plastic work, even for normal impact, and far exceeded those due to plastic work for highly oblique impact. Average frictionally induced temperature rises near $900 \mathrm{~K}$ were predicted for $\phi^{\circ}=0^{\circ}$ increasing to $4400 \mathrm{~K}$ for $\phi^{\circ}=80^{\circ}$. These predictions collectively highlight the importance of properly describing friction as a local heating mechanism that 
may induce combustion of energetic particle clusters. This study has provided a foundation for systematically characterizing how other potentially important phenomena, such as wall deformation, particle phase change, and far-field particle interactions, can influence the near wall impact energetics of heterogeneous solids.

\subsection{Uniaxial Deformation Waves}

Predictions were obtained for the temporal and spatial partitioning of energy within piston supported uniaxial deformation waves propagating through large ensembles of close-packed, micron size particles. Particular emphasis was placed on analyzing deformation induced fluctuations in thermomechanical fields resulting from porosity and particle interactions within the mesostructure. This study is relevant to accidental particle-scale and bulk ignition of energetic solids by weak, low pressure impact $(0.4-3 \mathrm{Km} / \mathrm{s}$ and $0.1-1 \mathrm{GPa})$. The importance of friction and plastic work as potential hot-spot formation mechanisms was characterized for piston impact speeds of $v_{p}=50 \mathrm{~m} / \mathrm{s}$ to $500 \mathrm{~m} / \mathrm{s}$. Bulk deformation wave structure was examined by suitably averaging meso-scale field predictions. Sensitivity studies were performed to examine the influence of friction coefficient, viscosity and initial particle size distribution on the thermomechanical fields within the wave structure.

Bulk wave structures having a mostly elastic precursor region, followed by a mostly plastic region in which the porosity is significantly reduced, were predicted for all cases considered in this study, though the lengths of these regions varied with piston speed. For low piston speeds $\left(v_{p}<100 \mathrm{~m} / \mathrm{s}\right)$, the wave structure was predicted to be thick and dispersed $(\sim 30$ average particle diameters) due to effective stress bridging between particles with very little compaction (bulk volumetric deformation); here, compaction was shown to be largely due to rigid particle rearrangement rather than plastic void collapse. For $v_{p}=50 \mathrm{~m} / \mathrm{s}$, the average solid volume fraction marginally increased from its initial average value of $\bar{\phi}_{s, 0}=0.835$ to a final quasi-equilibrium value of $\bar{\phi}_{s, f}=0.852$. The particle ensemble was fully compacted $\left(\bar{\phi}_{s, f}=1\right)$ for $v_{p} \geq 400 \mathrm{~m} / \mathrm{s}$ due to significant particle plastic deformation associated with void collapse. For these speeds, the 
wave structure consisted of a very thin precursor zone ( $\sim 1$ average particle diameter $)$ followed by a slightly thicker plastic zone ( $~ 3$ average particle diameters). Close examination of the particle dynamics incidated that deformation wave structure strongly depends on material mesostructure for low piston speeds $\left(v_{p} \leq 100 \mathrm{~m} / \mathrm{s}\right)$, and is insensitive to meso-structure for high speeds $\left(v_{p} \geq 400 \mathrm{~m} / \mathrm{s}\right)$. For piston speeds approximately between $100 \mathrm{~m} / \mathrm{s}$ and $400 \mathrm{~m} / \mathrm{s}$, local spatial variations in porosity within the meso-structure, which depend on the initial particle size distribution and packing configuration, induce large amplitude spatial fluctuations in thermomechanical fields behind the wave.

Predicted average pressures (0.08-1.6 GPa for $\left.v_{p}=50-500 \mathrm{~m} / \mathrm{s}\right)$ and effective plastic strains (0.001-0.11 for $\left.v_{p}=50-500 \mathrm{~m} / \mathrm{s}\right)$ behind the compaction wave increased with piston speed. High amplitude, high frequency spatial fluctuations in these quantities were also predicted due to wave reflections within and between particles. Meso-scale stress contours showed that stress is transmitted in preferential directions within the wave by interparticle contact giving rise to high-stress pathways within the meso-structure that are mostly aligned in the axial direction. Particles involved in these stress chains experienced significant stress concentrations and plastic work near contact surfaces, even for low impact speeds. Wave speed predictions showed that the precursor region of the compaction zone propagates faster then the trailing plastic region for $v_{p}<400 \mathrm{~m} / \mathrm{s}$, resulting in spatially separated structures. The waves coalesce at higher piston speeds. Predicted Hugoniot curves (wave speed vs. piston speed, and pressure vs. piston speed) qualitatively agreed with measured data.

Plastic work was shown to be the dominant energy dissipation mechanism in that it constitutes a substantial fraction of total system energy (25-35\% for $\left.v_{p}=50-500 \mathrm{~m} / \mathrm{s}\right)$, whereas friction work constitutes a smaller fraction $\left(2-1 \%\right.$ for $\left.v_{p}=50-500 \mathrm{~m} / \mathrm{s}\right)$. The maximum plastic and friction work occurred close to the piston boundary due to material and geometry mismatches between the rigid, planar piston and the initially circular particles. Close to the piston, friction work increased from $0.2 \mathrm{~J} / \mathrm{m}$ to $1.3 \mathrm{~J} / \mathrm{m}$ for $v_{p}=50$ to $500 \mathrm{~m} / \mathrm{s}$, whereas plastic work increased from $0.3 \mathrm{~J} / \mathrm{m}$ to $45 \mathrm{~J} / \mathrm{m}$. Plastic work performed by the wave influenced considerably more mass than friction 
work. For low piston speeds, only $7 \%$ of mass within the compaction zone experienced friction work, whereas $20 \%$ experienced plastic work; these percentages increased to $17 \%$ and $100 \%$ for $v_{p}=500 \mathrm{~m} / \mathrm{s}$, respectively. Predictions for mass-specific friction and plastic work highlighted two important results. First, mass-specific friction work dominates plastic work for $v_{p}=50 \mathrm{~m} / \mathrm{s}$, but they are comparable for $v_{p}=250 \mathrm{~m} / \mathrm{s}$ indicating that friction work may be more important than plastic work in producing hot-spots for low speed impact. Second, friction work is sensitive to details of the meso-structure, particularly for low speed impact. In these respects, it is likely that computational models used to describe reactive, high speed impact are more forgiving than those used to describe low speed impact.

Predicted maximum hot-spot temperatures far exceeded their average values, even for $v_{p}=$ $500 \mathrm{~m} / \mathrm{s}$ where a maximum average temperature of only $368 \mathrm{~K}$ was predicted; this average value which is well below the approximate ignition threshold for $\operatorname{HMX}\left(T_{i g} \approx 600 \mathrm{~K}\right)$. Maximum hotspot temperatures near $600 \mathrm{~K}$ were predicted for $v_{p}=50 \mathrm{~m} / \mathrm{s}$, which increased to near $1100 \mathrm{~K}$ and $1400 \mathrm{~K}$ for $v_{p}=250 \mathrm{~m} / \mathrm{s}$ and $500 \mathrm{~m} / \mathrm{s}$. Meso-scale temperature fields, illustrated by particle-scale finite element contours, indicated that hot-spots induced by both plastic and friction work are of sub-particle size. Frictionally induced hot-spots occurred along contact interfaces; plastically induced hot-spots occurred within particles in the vicinity of contact surfaces, and they were substantially larger in size and greater in number than frictional hot-spots.

Heated mass was predicted to steadily increase with distance behind the wave front until a quasi-steady value was reached at the end of the compaction zone, with minimal variation with position beyond that location. Minimal heating was predicted to occur within the precursor region of the compaction zone, resulting in $\Delta T \leq 5 \mathrm{~K}$ for all cases. Quasi-steady, local hot-spot mass fraction distribution curves behind the wave consisted of three parts based on temperature rise intervals $\Delta T_{b}$ and $\Delta T_{f}$. The first part included mass-fractions ranging from $10^{-1}$ and $10^{-3}$ that were heated through a temperature rise of $\Delta T_{b} \approx 30,100$, and $200 \mathrm{~K}$ for $v_{p}=50,250$, and $500 \mathrm{~m} / \mathrm{s}$. These hot-spots primarily resulted from plastic work occurring within the interior of particles. The second part of the distribution curves included mass-fractions ranging between $10^{-3}$ 
to $10^{-4}$ that were heated through a temperature rise between $\Delta T_{b}$ and $\Delta T_{f}$, where $\Delta T_{f} \approx 70,200$, and $400 \mathrm{~K}$ for $v_{p}=50,250$, and $500 \mathrm{~m} / \mathrm{s}$, respectively. These hot-spots resulted from a combination of plastic and friction work occurring in the vicinity of contact surfaces. The third part, or tail, of the distribution curves consisted of small mass-fractions $\left(m=10^{-5}\right)$ that experienced $\Delta T \geq \Delta T_{f}$. These hot-spots, which resulted from friction work, varied sporadically with axial position behind the wave due to spatially nonuniform heterogeneities within the meso-structure; they increased in number with piston speed. By comparing hot-spot contours obtained using the predicted temperature field to those estimated using an adiabatic plasticity analysis, where frictional effects and thermal conduction were ignored, for $v_{p}=50 \mathrm{~m} / \mathrm{s}$ revealed that only highly isolated mass (mass-fraction $\sim 10^{-5}$ ) experienced $\Delta T \geq 25 \mathrm{~K}$ due to plasticity. For this speed, almost all mass heated above $25 \mathrm{~K}$ was entirely due to frictional dissipation. For $v_{p}=250 \mathrm{~m} / \mathrm{s}$ and $500 \mathrm{~m} / \mathrm{s}$, almost all mass heated to $\Delta T \geq 300 \mathrm{~K}$ was due to a combination of plasticity and friction, or friction alone. These predictions collectively highlight that friction work is the more important hot-spot mechanism for low speed impact, and, though often ignored, is also significant for high speed impact.

Sensitivity studies examining the influence of the friction coefficient on the compaction wave structure were performed by varying the friction coefficient from zero to the baseline value of 0.25. Predictions from this study showed that the quasi-steady final solid volume fraction $\bar{\phi}_{s, f}$ increased marginally with decreasing friction coefficient due to increased mobility of the particles. Increasing the friction coefficient promoted stick behavior within the particle ensemble resulting in a "frictionally rigid" bed. Frictional stick decreased particle mobility at higher friction coefficients and promoted more efficient stress transmission between particles resulting in faster wave speeds. The predicted pressure and stress fields displayed minimal sensitivity to changes in friction coefficient. Plastic deformation was also shown to be insensitive to friction coefficient. Although the average temperatures are predicted to marginally increase with friction coefficient, the maximum hot-spot temperatures are shown to increase substantially. While maximum temperatures of only 360, 460 and $660 \mathrm{~K}$ were predicted for the frictionless 50,250 and $500 \mathrm{~m} / \mathrm{s}$ cases, respec- 
tively, temperatures close to 600,1100 and $1400 \mathrm{k}$ are predicted for the corresponding baseline cases. Hot-spot predictions showed that although the bulk of the hot-spot distributions varied minimally with friction coefficient, frictionally induced mass-fractions $\left(m=10^{-5}\right)$, which formed the high temperature end of the distributions, increased significantly in number with increase in friction coefficient. Predictions from this study indicated that while plastically induced hot-spots are insensitive to friction coefficient, frictionally induced hot-spot number density is influenced by the friction coefficient, with a larger friction coefficient resulting in more hot-spots with larger temperature rises.

Sensitivity studies examining the influence of the plastic viscosity on the compaction wave structure were performed by varying the viscosity from the baseline value of 100 to 10 . Lowering the viscosity parameter resulted in increased inelastic deformation rates following initial impact. The ensemble offered lesser resistance to compaction which resulted in larger quasi-steady final solid volume fraction $\bar{\phi}_{s, f}$ and marginally lower wave speeds. The predicted pressure field was shown to be insensitive to changes in viscosity. A lower viscosity value also resulted in lower Von Mises stresses $\overline{\boldsymbol{\tau}}_{e}$ but larger plastic strains $\bar{\epsilon}_{p}$. Subsequently, the predicted plastic heating, which is the product of $\overline{\boldsymbol{\tau}}_{e}$ and $\bar{\epsilon}_{p}$, was shown to be only marginally sensitive to changes in viscosity. The predicted average temperatures displayed similar qualitative and quantitative trends for both viscosities. However, the maximum temperatures induced within the bed for the lower viscosity cases were shown to be substantially lower than the baseline cases at high piston speeds $\left(v_{p}>\right.$ $100 \mathrm{~m} / \mathrm{s})$, but comparable at lower piston speeds. Maximum temperatures of only 620, 910 and $950 \mathrm{~K}$ were predicted for $v_{p}=50,250$ and $500 \mathrm{~m} / \mathrm{s}$ cases, respectively. Predictions for the hot-spot distributions showed that the bulk of the distributions were insensitive to the viscosity parameter. However, substantial differences were observed in frictionally induced hot-spots. These hot-spots decreased in number and had lower temperature rises for the lower viscosity case. Predictions from this study showed that although viscosity has minimal influence on the hot-spots induced due to plastic heating, low viscosity values substantially reduce the number and the temperatures of frictionally induced hot-spots, especially at high piston speeds $\left(v_{p}>100 \mathrm{~m} / \mathrm{s}\right)$. 
Sensitivity of the thermomechanical fields within deformation wave structures to the assumed particle size distribution (PSD) was demonstrated by performing compaction studies on an alternate particle ensemble with the same initial porosity but a wider PSD. Predictions for the mechanical response of both ensembles displayed similar qualitative and quantitative trends. A wider PSD resulted in marginally larger quasi-steady final solid volume fraction $\bar{\phi}_{s, f}$ and marginally lower wave speeds due to increased mobility of the smaller particles. For a given piston speed, the predicted quasi-steady final compacted pressure of the ensemble and each individual particle size class were shown to be the same for both ensembles. However, the stress state within the smaller particles displayed larger spatial fluctuations than the larger particles. The plastic response and the average temperature response of the ensembles was shown to be only marginally sensitive to PSD. The predicted average temperature response for different particle size classes indicated that the smaller particles have larger average temperatures and therefore greater thermal energy per unit mass. The frictional response was shown to be highly sensitive to PSD. A wider PSD enhanced frictional dissipation leading to greater frictional heating rates. Subsequently, for this ensemble, the predicted maximum temperatures increased to 875,1570 and $1870 \mathrm{~K}$ for $v_{p}=50$, 250 and $500 \mathrm{~m} / \mathrm{s}$ case, respectively. Predicted hot-spot distributions showed that although the bulk of the distribution is insensitive to PSD, the high temperature tail end of the distributions is highly sensitive. For all piston speeds, frictionally induced hot-spots increased in number and had larger temperatures for the wider PSD ensemble. Predictions for the hot-spot distribution for each particle size indicated that while the smaller particles had larger average temperatures, the larger particles had the maximum local temperature rises and contributed to the high temperature end of the distribution. These predictions collectively indicate that the frictional response of the particle ensemble is much more sensitive to PSD than the plastic response. The predictions also suggested that the larger particles play a significant role in combustion initiation whereas the smaller particles are important for reaction propagation. 


\subsection{Future Work}

Many modeling improvements can be made as warranted to address more complex phenomena. In particular, various material properties for HMX, such as yield strength and specific heat, exhibit a strong temperature dependence which could affect hot-spot formation. More accurate descriptions than used here can be included in the analysis to account for these functional de-

pendencies. Also, HMX is known to melt prior to ignition; therefore, a complete description of hot-spot formation should account for latent heat and multiphase lubrication effects. However, because the current method is Lagrangian, it may be difficult to explicitly model phase change and fluid flow at contact interfaces. Further, both hot-spot mass fraction and temperature are limited by thermal conduction which is difficult to numerically resolve for high speed impact without the use of excessively (and perhaps prohibitively) fine grids. As such, sub-grid models are likely needed to accurately describe the effect of thermal conduction on the tribology of granular energetic solids. Lastly, powdered metals, such as aluminium and magnesium, are often mixed with high-explosives to form high energy formulations. Inclusion of such metals enhances post-detonation blasts due to their high energy output when oxidized $(\approx 14.719 .6 \mathrm{MJ} / \mathrm{kg})$. The meso-scale model can be easily extended to analyze the impact response of explosive-metal mixtures. 


\section{References}

[1] Andersen WH (1981) Role of friction coefficient in the frictional heating ignition of explosives. Propellants and Explosives. 6: 17-23

[2] Armero F, Petocz E (1998) Formulation and analysis of conserving algorithms for fritionless dynamic contact/impact problems. Computational Methods in Applied Mechanics and Engineering. 158: 269-300

[3] Armero F, Petocz E (1999) A new dissipative time-stepping algorithm for frictional contact problems: formulation and analysis. Computational Methods in Applied Mechanics and Engineering. 179: 151-178

[4] Baer MR, Nunziato JW (1986) A two-phase mixture theory for the deflagration-todetonation transition in reactive granular materials. International Journal of Multiphase Flow. 12: 861-889

[5] Baer MR, Hertel ES, Bell RL (1996) Multidimensional DDT modeling of energetic materials. AIP Conference Proceedings: Shock Compression of Condensed Matter. 370: 433-436

[6] Baer MR, Kipp ME, van Swol F (1998) Micromechanical modeling of heterogeneous energetic materials. Eleventh International Detonation Symposium, Snowmass, CO. 788-797

[7] Baer MR (1999) Computational modeling of heterogeneous reactive materials at the mesoscale. AIP Conference Proceedings: Shock Compression of Condensed Matter, Snowbird, UT. 27-33

[8] Baer MR, Trott WM (2001) Mesoscale descriptions of shock-loaded heterogeneous Porous Materials. AIP Conference Proceedings: Shock Compression of Condensed Matter. 713-716

[9] Baer MR (2002) Modeling heterogeneous energetic materials at the mesoscale. Thermochemika Acta. 384(1/2): 351-367

[10] Bardenhagen SG, Brackbill JU (1998) Dynamic stress bridging in granular material. Journal of Applied Physics. 83(11): 5732-5740

[11] Bardenhagen SG, Roessig KM, Byutner O, Guilkey JE, Bedrov D, Smith GD (2002) Direct numerical simulations of weak shocks in granular materials. Twelfth International Symposium on Detonation, San Diego, CA.

[12] Bathe KJ, Bouzinov PA (1997) On the constraint function method for contact problems. Computers and Structures. 64(5/6): 1069-1085

[13] Bdzil JB, Menikoff R, Son SF, Kapila AK, Stewart DS (1999) Two-phase modeling of deflagration-to-detonation transition in granular materials: A critical examination of modeling issues. Physics of Fluids. 11(2): 378-402

[14] Belytschko T, Lu YY, Gu L (1994) Element free Galerkin methods. International Journal of Numerical Methods in Engineering. 37: 229-256 
[15] Benson DJ, Nellis WJ (1993) Numerical simulation of the shock compaction of copper powder. High-Pressure Science and Technology, Colorado Springs, CO. 1243-1246

[16] Benson DJ (1994) An analysis by direct numerical simulation of the effects of particle morphology on the shock compaction of copper powder. Modeling and Simulation in Material Science and Engineering. 2(3A): 535-550

[17] Benson DJ, Nestorenko VF, Jonsdottir F (1995) Micromechanics of shock deformation of granular materials. AIP Conference Proceedings: Shock Compression of Condensed Matter, New York. 603606

[18] Benson DJ (1995) The calculation of the shock velocity - particle velocity relationship for a copper powder by direct numerical simulation. Wave Motion. 21: 85-99

[19] Benson DJ, Tong W, Ravichandran G (1995) Particle-level modeling of dynamic consolidation of T0-SiC powders. Modelling and Simulation in Materials Science and Engineering. 3: $771-796$

[20] Benson DJ (1997) The numerical simulation of dynamic compaction of powders. HighPressure Shock Compression of Solids IV: Response of Highly Porous Solids to Shock Compression, Springer-Verlag, New York. 9: 233-255

[21] Benson DJ, Nesterenko VF, Jonsdottir F, Meyers MA (1997) Quasistatic and dynamic regimes of granular material deformation under impulse loading. Journal of Mechanics and Physics of Solids. 45: 1955-1999

[22] Benson DJ, Conley P (1999) Eulerian finite-element simulations of experimentally acquired HMX microstructures. Modelling and Simulation in Material Science and Engineering. 7: 333-354

[23] Bonet J, Wood RD (2000) Nonlinear continuum mechanics for finite element analysis. Cambridge university press, Cambridge, UK.

[24] Borg JP, Vogler TJ (2008) Mesoscale calculations of the dynamic behavior of granular ceramic. International Journal of Solids and Structures. 45: 1676-1696

[25] Bowden FP, Gurton OA (1949) Birth and growth of explosion in liquids and solids initiated by impact and friction. Proceedings of the Royal Society of London. Series A, Mathematical and Physical Sciences. 198: 350-372

[26] Bowden FP, Gurton OA (1949) Initiation of solid explosives by impact and friction: The influence of grit. Proceedings of the Royal Society of London. Series A, Mathematical and Physical Sciences. 198: 337-349

[27] Bowden FP, Yoffe AD (1952) Initiation and growth of explosions in liquids and solids. Cambridge University Press, Cambridge.

[28] Brach RM (1991) Mechanical impact dynamics: rigid body collisions. John Wiley and Sons, New York. 
[29] Brach RM (1998) Formulation of rigid body impact problems using generalized coefficients. International Journal of Engineering Science. 36: 61-71

[30] Brackbill JU, Pracht WE (1973) An implicit, almost-Lagrangian algorithm for magnetohydrodynamics. Journal of Computational Physics. 13: 455-482

[31] Butler PB, Krier H (1984) Analysis of deflagration to shock to detonation transition (DSDT) in porous energetic solid propellants. Proceedings of AGARD Conference on Energetic Propellants, Conference Proceedings, Lisse, The Netherlands. 367: 5-10.

[32] Case S, Horie Y (2005) Mesoscale modeling of the response of alumina. Shock Compaction of Condensed Matter. 299-302

[33] Case S, Horie Y (2007) Discrete element simulation of shock wave propagation in polycrystalline copper. Journal of Mechanics and Physics of Solids. 55: 589-614

[34] Chaudhri MM, Field JE (1974) The role of rapidly compressed gas pockets in the initiation of condensed explosives. Proceedings of the Royal Society-A. 340: 113-128

[35] Chaudhri MM (1974) Stab initiation of explosives. Nature. 263(169)

[36] Chaudhri MM (1992) Photographic evidence for ignition by friction in a deflagrating explosive single crystal. Journal of Applied Physics. 25: 552-557

[37] Conley PA, Benson DJ (1999) An estimate of the linear strain rate dependence of Octahydro1,3,5,7-tetranitro-1,3,5,7-tetrazocine. Journal of Applied Physics. 86: 6717-6728

[38] Copp JI, Napier SE (1948) The sensitiveness of explosives. Philosophical Transactions of the Royal Society. 241: 198-296

[39] Deguet A, Joukhadar A, Laugier C (1998) A collision model for deformable bodies. IEEE International Conference on Robotics and Automation. 1: 636-641

[40] Dick JJ, Hooks DE, Menikoff R, Martinez AR (2004) Elastic-plastic wave profiles in cyclotetramethylene tetranitramine crystals. Journal of Applied Physics. 96(1): 374-379

[41] Dienes JK (1984) Frictional hot spots and propellant sensitivity. Proceedings of Materials Research Society Symposium. 373-383

[42] Dobratz BM (1974) Properties of chemical Explosives and simulants. UCRL-51319 (Revision 1), Lawrence Livermore Laboratory, Livermore, CA.

[43] Farahani K, Mo DM, Vafai A (2000) A solution method for general contact-impact problems. Computer Methods in Applied Mechanics and Engineering. 187: 69-77

[44] Field JE, Bourne NK, Palmer SJP, Walley SM (1992) Hot-spot ignition mechanisms for explosives and propellants. Philosophical Transactions of the Royal Society of London. 339: 269-283 
[45] Fredrich JT, Menendez B, Wong TF (1995) Imaging the pore structure of geomaterials. Science. 268: 276-279

[46] Frey RB (1981) The initiation of explosive charges by rapid shear. Seventh Symposium on Detonation. 36-42

[47] Gonthier KA, Powers JM (2000) A high resolution numerical method for a two-phase model of deflagration-to-detonation transition. Journal of Computational Physics. 163: 376-433

[48] Gonthier KA (2003) Modeling and analysis of reactive compaction for granular energetic solids. Combustion Science and Technology. 175: 1679-1709

[49] Gonthier KA (2003) Predictions for weak mechanical ignition of strain hardened granular explosive. Journal of Applied Mechanics. 95(7)

[50] Gonthier KA, Jogi V (2005) Multiscale shock heating analysis of a granular explosive. ASME Journal of Applied Mechanics. 72: 538-552

[51] Grady DE, Kipp ME (1985) The growth of inhomogeneous thermoplastic shear. Proceedings of the International Conference on Mechanical and Physical Behavior of Materials Under Dynamics Loading, Paris.

[52] Greenaway MW (2005) Measurement of intergranular stress and porosity during dynamic compaction of porous beds of cyclotetramethylene tetranitramine. Journal of Applied Physics. 97(9)

[53] Groot RD, Warren PB (1997) Dissipative particle dynamics: bridging the gap between atomistic and mesoscopic simulation. Journal of Chemical Physics. 107: 4423-4435

[54] Gropp W, Lusk E, Skjellum A (1999) Using MPI: portable parallel programming with the message-passing interface, MIT Press.

[55] Hae-Jin C, Austin R, Allen JK, McDowell DL, Mistree F, Benson DJ (2005) An approach for robust design of reactive powder metal mixtures based on non-deterministic micro-scale shock simulation. Journal of Computer-Aided Materials Design. 12: 57-85

[56] Heinstein MW, Mello FJ, Attaway SW, Laursen TA (2000) Contact-impact modeling in explicit transient dynamics, Computer Methods in Applied Mechanics and Engineering. 187: $621-640$

[57] Hirt CW, Amsden AA, Cook JL (1974) An arbitrary Lagrangian-Eulerian computing method for all flow speeds. Journal of Computational Physics. 14: 227-254

[58] Hoogerbrugge PJ, Koelman JMVA (1992) Simulating microscopic hydrodynamics phenomena with dissipative particle dynamics. Europhysics Letter. 19: 155-170

[59] Howe P, Frey R, Taylor B, Boyle V (1976) Shock initiation and critical energy concept. Proceedings of the Sixth International Symposium on Detonation, ACR-221, ONR, Arlington, VA. $11-19$ 
[60] Do IPH, Benson DJ (2001) Micromechanical modeling of shock-induced chemical reactions in heterogeneous multi-material powder mixtures. International Journal of Plasticity. 17: 641-668

[61] Johnson JN, Jones OE (1970) Dislocation dynamics and single-crystal constitutive relations: Shock-wave propagation and precursor decay. Journal of Applied Physics. 59(6): 2330-2339

[62] Johnson JN, Tang PK, Forest CA (1985) Shock wave initiation of heterogeneous reactive solids. Journal of Applied Physics. 57(4323)

[63] Johnson KL (1985) Contact mechanics, Cambridge University Press, Cambridge.

[64] Johnson JN, Tang PK, Forest CA (1985) Shock-wave initiation of heterogeneous reactive solids. Journal of Applied Physics. 57(4323)

[65] Kang J, Butler PB, Baer MR (1992) A thermomechanical analysis of hot spot formation in condensed-phase energetic materials. Combustion and Flame. 89(117)

[66] Kapila AK, Menikoff R, Bdzil JB, Son SF, Stewart DS (2001) Two-phase modeling of deflagration-to-detonation transition in granular materials: Reduced equations. Physics of Fluids. 13(10): 3002-3024

[67] Keshavarz S, Khoei AR, Khaloo AR (2007) Contact friction simulation in powder compaction process based on the penalty approach. Materials and Design. 29: 1199-1211

[68] Khasainov A, Borisov AA, Ermolaev BS, Korotkov AI (1981) Two-phase visco-plastic model of shock initiation of detonation in high density pressed explosives. Proceedings of the 7 th Symposium on Detonation, Annapolis, MD. 435-447.

[69] Khoei AR (2002) Numerical simulation of powder compaction processes using an inelastic finite element analysis. Materials and Design. 23: 523-529

[70] Khoei AR, Keshavarz S, Khaloo AR (2008) Modeling of large deformation frictional contact in powder compaction processes. Applied Mathematical Modeling. 32: 775-801

[71] Khoei AR, Keshavarz S, Khaloo AR (2007) Modeling of large deformation frictional contact in powder compaction processes. Applied Mathematical Modeling. 32: 775-801

[72] Kim SW (1999) Contact dynamics and force control of flexible multi-body systems. Ph.D. Thesis, Department of Mechanical Engineering, McGill University, Montreal.

[73] Kipp ME (1985) Modeling granular explosives detonation with shear band concepts. Proceedings of the 8th Symposium on Detonation, Albuquerque, NM. 35-41

[74] Kraus PR, Kumar V (1987) Compliant contact models for rigid body collisions. IEEE International Conference on Robotics and Automation. 2: 1382-1387

[75] Krien G, Licht HH, Zierath J (1973) Thermochemical investigation of nitramines. Thermochimica Acta. 6: 465-472 
[76] Kumar DR, Kumar RK, Philip PK (1998) Simulation of dynamic compaction of metal powders. Journal of Applied Mechanics. 85(2): 767-775

[77] Laursen TA (1999) On the development of thermodynamically consistent algorithms for thermomechanical frictional contact. Computational Methods in Applied Mechanics and Engineering. 177: 273-287

[78] Laursen TA (2003) Computational contact and impact mechanics, Springer-Verlag, Berlin.

[79] Lee EL, Tarver CM (1980) Phenomenological model of shock initiation in heterogeneous explosives. Physics of Fluids. 23: 2362-2372

[80] Lewis RW, Gethin DT, Yang XS, Rowe RC (2005) A combined finite-discrete element method for simulating pharmaceutical powder tableting. International Journal for Numerical Methods in Engineering. 62(7): 853-869

[81] Libersky LD, Petschek AG, Peterson PA (1993) Calculation of reactive flow using smoothed particle hydrodynamics. Tenth International Symposium on Detonation, Boston, MA. 12: 199-203

[82] Lim CT, Stronge WJ (1999) Oblique elastic-plastic impact between rough cylinders in plane strain. International Journal of Engineering Science. 37: 97-122

[83] Liu WK, Jun S, Zhang YF (1995) Reproducing kernel particle methods. International Journal of Numerical Methods in Engineering. 20: 1081-1106

[84] Lowe CA, Greenaway MW (2005) Compaction processes in granular beds composed of different particle sizes. Journal of Applied Physics. 98(12): 123519.1-123519.12

[85] Lowe CA, Longbottom AW (2006) Effect of particle distribution on the compaction behavior of granular beds. Physics of Fluids. 18(6): 066101.1-066101.16

[86] Lubarda VA (2002) Elastoplasticity theory. CRC Mechanical Engineering, Boca Raton.

[87] Ma O (1995) Contact dynamics modeling for the simulation of the space station manipulators handling payloads. IEEE International Conference on Robotics and Automation. 2: 12521358

[88] Mader CL, Forest CA (1976) Two Dimensional Homogeneous and Heterogeneous Wave Propagation. Technical Report LA-6259, Los Alamos Scientific Laboratory.

[89] Massoni J, Samuel R, Baudin G (1999) A mechanistic model for shock initiation of solid explosives. Physics of Fluids. 11(3): 710-736

[90] McAfee JM, Asay BW, Campbell W, Ramsay JB (1989) Deflagration to detonation transition in granular HMX. Proceedings of the Ninth (International) Detonation Symposium. $265-278$

[91] Mader CL (1998) Numerical modeling of explosives and propellants. CRC Press, Baca Raton, FL. Second Ed. 
[92] Marsh S (1980) LASL shock hugoniot data. University of California Press.

[93] McGlaun JM, Thompson SL, Elrick MG (1990) CTH: a three-dimensional shock wave physics code. International Journal of Impact Engineering. 10: 351-360

[94] Meng XN, Laursen TA (2002) Energy consistent algorithms for dynamic finite deformation plasticity. Computational Methods in Applied Mechanics and Engineering. 191: 1639-1675

[95] Menikoff R, Kober E (1999) Compaction waves in granular HMX. Los Alamos Report, LA13546-MS, Los Alamos National Laboratory, Los Alamos, New Mexico.

[96] Menikoff R, Sewell TD (2002) Constituent properties of HMX needed for mesoscale simulations. Combustion Theory and Modeling. 6: 103-125

[97] Menikoff R (2003) Pore Collapse and Hot Spots in HMX. APS Topical Conference-Shock Compression of Condensed Matter, Portland, Oregon.

[98] Menikoff R, Dick JJ, Hooks DE (2005) Analysis of wave profiles for single-crystal cyclotetramethylene tetranitramine. Journal of Applied Physics. 97(2): 023529.1-023529.6

[99] Merzhievsky LA, Tyagelsky AV (1994) Modeling of dynamic compression of porous iron. Combustion, Explosion and ShockWaves. 30: 522-530

[100] Merzhievsky LA, Tyagelsky AV (1995) Modeling of shock compression of porous media. Shock Compression of Condensed Matter, Proceedings of the American Physical Society Topical Group on Shock Compression of Condensed Matter, Seattle, WA. 303-306

[101] Meyer R, Khler J, Homburg A (2007) Explosives. Wiley-VCH Sixth Ed.

[102] Monagham JJ (1992) Smoothed particle hydrodynamics. Annual Revision of Astronomy and Astrophysics. 30: 543-574

[103] Munjiza A, Andrews KRF (2000) Discretized penalty function method in combined finitediscrete element analysis. International Journal of Numerical Methods in Engineering. 49: $1495-1520$

[104] Munjiza A, Andrews KRF (2000) Penalty function method in combined finite-discrete element systems comprising large number of separate bodies. International Journal of Numerical Methods in Engineering. 49: 1377-1396

[105] Munjiza A (2004) The combined finite-discrete element method. John Wiley and Sons, New York.

[106] Munjiza A, Andrews KRF (1998) NBS contact detection algorithm for bodies of similar size. International Journal of Numerical Methods in Engineering. 43(1): 131-149.

[107] Negreskul SI, Psakhie SG, Korostelev SY (1989) The simulation of explosive compaction of powder by element dynamics. Shock Compression of Condensed Matter, Albuquerque, NM. $233-236$ 
[108] Ornellas DL, Carpenter JH, Gunn SR (1966) Detonation calorimeter and results obtained with Pentaerythritol Tetranitrate (PETN). Review of Scientific Instruments. 37: 907-912

[109] Pantuso D, Bathe K-J, Bouzinov PA (2000) A finite element procedure for the analysis of thermomechanical solids in contact. Computers and Structures. 75: 551-573

[110] Papadopoulos P, Solberg JM (1998) A Lagrange multiplier method for the finite element solution of frictionless contact problems. Mathematical and Computer Modeling. 28: 373-384

[111] Parton Y (1986) Elastic precursor decay calculation. Journal of Applied Physics. 59(8): 2716-2727

[112] Pietrzak G, Curnier A (1999) Large deformation frictional contact mechanics-continuum formulation and augmented Lagrangian treatment. Computational Methods in Applied Mechanics and Engineering. 177: 351-381

[113] Plaksin I, Compos J, et al. (2002) Detonation study of energetic micro-samples. Twelfth International Detonation Symposium, San Diego, California.

[114] Powers JM (2004) Two-phase viscous modeling of compaction of granular materials. Physics of Fluids. 16: 2975-2990

[115] Powers JM (2006) Review of multiscale modeling of detonation. Journal of Propulsion and Power. 22: 1217-1229

[116] Rae PJ, Goldrein HT, Palmer SJP, Field JE, Lewis AL (2002) Quasi-static studies of the deformation and failure of beta-HMX based polymer bonded explosives. Proceedings of the Royal Society of London. 458: 743-762

[117] Randles PW, Libersky LD (1996) Smoothed particle hydrodynamics: some recent improvements and applications. Computational Methods in Applied Mechanics and Engineering. 139 : 375-408

[118] Refaat MH, Meguid SA (1998) New strategy for the solution of frictional contact problems. International Journal for Numerical Methods in Engineering. 43(6): 1053-1068

[119] Rieger A, Wriggers P (2004) Adaptive methods for thermomechanical coupled contact problems. International Journal for Numerical Methods in Engineering. 59: 871-894

[120] Sheffield SA, Gustavsen RL, et al. (1993) Particle velocity and stress measurements in low density HMX. High-Pressure Science and Technology, Colorado Springs, CO. 1377-1380

[121] Sheffield SA, Gustavsen RL, Anderson MU (1997) Shock loading of porous high explosives. High-Pressure Shock Compression of Solids IV, Springer-Verlag.

[122] Solberg JM, Papadopoulos P (1998) A finite element method for contact/impact. Finite Elements in Analysis and Design. 30: 297311 
[123] Son SF, Asay BW, Bdzil JB, Kober EM (1995) Reaction rate modeling in the deflagration to detonation transition of granular energetic materials. Presented at the MRS Meeting, Boston, Massachusetts.

[124] Stewart DS, Asay B, Prasad K (1994) Simplified modeling of transition to detonation in porous energetic materials. Physics of Fluids. 6: 2515-2534

[125] Tanemura M (1992) Models and simulations of random structure of particles. Acta Stereology. 11(I): 41-52

[126] Tarver CM, Chidester SK, Nichols AL (1996) Critical conditions for impact-and shockinduced hot spots in solid explosives. Journal of Physical Chemistry. 100(14): 5794-5799

[127] Trott WM, Baer MR, Castaneda JN, Chhabildas LC, Asay JR (2007) Investigation of the mesoscopic scale response of low-density pressings of granular sugar under impact. Journal of Applied Physics. 101(2): 024917.1-024917.21

[128] Underwood EE (1970) Quantitative Stereology. Addison-Wesley, Reading, MA.

[129] Vogler TJ, Lee MY, Grady DE (2007) Static and dynamic compaction of ceramic powders. International Journal of Solids and Structures. 44: 636-658

[130] Vukobratovic MK, Potkonjak V (1999) Dynamics of contact tasks in robotics. Part I: General model of robot interacting with environment. Mechanism and Machine Theory. 34: 923-942

[131] Wellington SL, Vinegar JJ (1987) X-ray computed tomography. Journal of Petroleum Technology. 39: $885-898$

[132] Whitworth NJ, Maw JR (2000) Modelling 'hot-spot' initiation in heterogeneous solid explosives. In APS Topical Conference on Shock Compression of Condensed Matter, Proceedings of the American Physical Society Topical Conference, Snowbird, UT. 887-890.

[133] Williamson RL and Berry RA (1986) Shock waves in condensed matter. Plenum, New York, NY.

[134] Williamson RL (1990) Parametric studies of dynamic powder consolidation using a particlelevel numerical model. Journal of Applied Physics. 68(3): 1287-1296

[135] Winter RE, Field JE (1975) The role of localized plastic flow in the impact initiation of explosives. Proceedings of the Royal Society-A. 343: 399-413

[136] Yano K, Horie Y (1999) Discrete-element modeling of shock compression of polycrystalline copper. Physics Review B. 59(21): 13672-13680

[137] Zavaliangos A (2002) A multiparticle simulation of powder compaction using finite element discretization of individual particles. Materials Research Society Symposium - Proceedings. 731: $169-176$ 


\section{Vita}

Rohan Panchadhar is from the city of Hyderabad, Andhra Pradesh, in India. After graduating from Saint Mary's Junior College in Hyderabad, Andhra Pradesh, in 1999, he enrolled in Jawaharlal Nehru Technological University in Hyderabad. He earned a Bachelor of Technology degree in mechanical engineering in 2003. In August of 2003 he enrolled in Louisiana State University, Baton Rouge, as a Ph.D. student with a full assistantship and a graduate student supplement scholarship. Over the course of his education at LSU he presented his work in several international conferences. He was awarded the best presentation and best paper award at the 23rd Southeastern Conference on Theoretical and Applied Mechanics, Mayaguez, Puerto Rico. He has also published his work in several international journals as well as conference proceedings. He is a candidate for the Ph.D. degree in Mechanical Engineering to be awarded in August 2009. 